\title{
On the Morse index of branched Willmore spheres in 3-space
}

\author{
Alexis Michelat ${ }^{1}$ \\ Received: 24 June 2019 / Accepted: 28 February 2021 / Published online: 25 June 2021 \\ (c) The Author(s) 2021
}

\begin{abstract}
We develop a general method to compute the Morse index of branched Willmore spheres and show that the Morse index is equal to the index of certain matrix whose dimension is equal to the number of ends of the dual minimal surface (when the latter exists). As a corollary, we find that for all immersed Willmore spheres $\vec{\Phi}: S^{2} \rightarrow \mathbb{R}^{3}$ such that $W(\vec{\Phi})=4 \pi n$, we have $\operatorname{Ind}_{W}(\vec{\Phi}) \leq n-1$.
\end{abstract}

Mathematics Subject Classification 35J35 - 35R01 - 49Q05 - 49Q10 - 53A05 - 53A10 . $53 \mathrm{~A} 30 \cdot 53 \mathrm{C} 42 \cdot 58 \mathrm{E} 15$

\section{Contents}

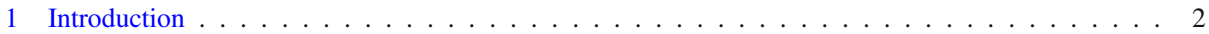

2 Main results . . . . . . . . . . . . . . . . . . . . . . . . 4

3 The second derivative of the Willmore energy as a renormalised energy . . . . . . . . . . . 6

4 Explicit description of the admissible variations . . . . . . . . . . . . . . . . . . . 9

5 Decomposition of the renormalised energy . . . . . . . . . . . . . . . . . . . . 17

5.1 Estimate of the singular energy of the minimisers . . . . . . . . . . . . . . . 18

5.2 Local estimates near the ends . . . . . . . . . . . . . . . . . . . . . . . . 25

5.3 Indicial roots analysis: case of embedded ends . . . . . . . . . . . . . . . . . . . . . 28

5.4 Indicial roots analysis: case of ends of higher multiplicity . . . . . . . . . . . . . . . . . 36

6 Renormalised energy for minimal surfaces with embedded ends . . . . . . . . . . . . . . . . . . 42

6.1 Explicit computation of the singular energy . . . . . . . . . . . . . . . . . . . . . . . . .

6.2 Renormalised energy identity . . . . . . . . . . . . . . . . . . . . . 53

7 Equality of the Morse index for inversions of minimal surfaces with embedded ends . . . . . . . . . 61

8 Renormalised energy for ends of arbitrary multiplicity . . . . . . . . . . . . . . . . . . 65

9 Morse index estimate for Willmore spheres in $S^{4} \ldots \ldots \ldots \ldots$. . . . . . . . . . . . . 72

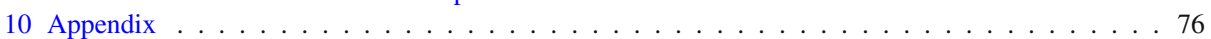

10.1 Estimates for some weighted elliptic operators . . . . . . . . . . . . . . . . . 76

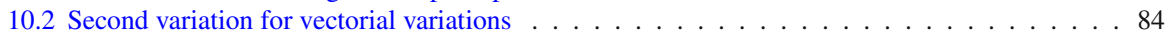

10.3 Formulae for inversions of (minimal) surfaces . . . . . . . . . . . . . . . . . . . . 92

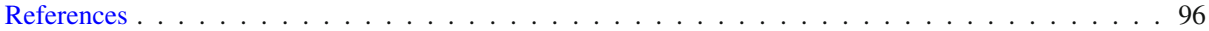

Communicated by A. Malchiodi.

$\bowtie$ Alexis Michelat

michelat@maths.ox.ac.uk

1 Department of Mathematics, ETH Zentrum, 8093 Zürich, Switzerland 


\section{Introduction}

It was proposed by Tristan Rivière in [36] to study the topology of immersions of surfaces into Euclidean space by means of a quasi-Morse function (say $\mathscr{L}$ ). Fix a closed surface $M^{2}$ and let $\operatorname{Imm}\left(M^{2}, \mathbb{R}^{n}\right)$ be the space of smooth immersions $\vec{\Phi}: M^{2} \rightarrow \mathbb{R}^{n}$. We look for a Lagrangian $\mathscr{L}: \operatorname{Imm}\left(M^{2}, \mathbb{R}^{n}\right) \rightarrow \mathbb{R}$ satisfying the following properties for all $\vec{\Phi}: M^{2} \rightarrow \mathbb{R}^{n}:$

(1) $\mathscr{L}(\vec{\Phi}+\vec{c})=\mathscr{L}(\vec{\Phi})$ for all $\vec{c} \in \mathbb{R}^{n}$ (translational invariance)

(2) $\mathscr{L}(R \vec{\Phi})=\mathscr{L}(\vec{\Phi})$ for all $R \in \mathrm{O}(n)$ (rotational invariance)

(3) $\mathscr{L}(\lambda \vec{\Phi})=\mathscr{L}(\vec{\Phi})$ for all $\lambda>0$ (scaling invariance).

Indeed, an immersion does not change geometrically when one translates, rotates or dilates it.

Now, assume that $n=3$. To an immersed surface one can attach two natural quantities: the principal curvatures $\kappa_{1}, \kappa_{2}$ (introduced by Euler in 1760 [12]) which are the maximum and the minimum of the curvatures of normal sections of the surface at a given point. Then we define the mean curvature $H$ and the Gauss curvature $K$ (introduced by Meusnier in 1776 [24]) by

$$
H=\frac{\kappa_{1}+\kappa_{2}}{2}, \text { and } K=\kappa_{1} \kappa_{2} .
$$

Thanks to the third property, $\mathscr{L}$ must be a quadratic expression of the principal curvatures (see also [30] for a more general study of conformal invariants of Euclidean space), which says that up to scaling

$$
\mathscr{L}(\vec{\Phi})=\int_{M^{2}}\left(H^{2}+\lambda K\right) d \operatorname{vol}_{g}
$$

for some $\lambda \in \mathbb{R}$, where $g=g_{\vec{\Phi}}=\vec{\Phi}^{*} g_{\mathbb{R}^{3}}$. Thanks to the Gauss-Bonnet theorem,

$$
\int_{M^{2}} K d \operatorname{vol}_{g}=2 \pi \chi\left(M^{2}\right)
$$

is a constant independent of the immersion. Therefore, up to constants, the only non-trivial such quasi-Morse function is

$$
\mathscr{L}(\vec{\Phi})=\int_{\Sigma} H^{2} d \operatorname{vol}_{g}
$$

which is generally denoted by $\mathscr{L}=W$ and is called the Willmore energy.

This Lagrangian actually first appeared in the work of Poisson in 1814 and Germain's third memoir of 1815 respectively in their work about elasticity [15,16,33]. It was considered by many geometers in the following years, including in important work of Navier [31]. For more information on the history in which these considerations about elasticity emerged, we refer to the comprehensive work of Todhunter [46]. Poisson was the first one to obtain the correct Euler-Lagrange equation, more than 100 years before Blaschke and Thomsen, who attributed it to Schadow in 1922 [5,45]. He also found in 1814 the first version of the Gauss-Bonnet theorem, and his student Rodrigues computed the following year the exact constant $4 \pi$ for ellipsoids, but unfortunately made a sign mistake and found $8 \pi$ for tori $[37,38]$. The famous memoir of Gauss on the subject of the curvature of surfaces appeared only in 1827 [14], and Gauss-Bonnet in a published form in 1848 [6].

This Lagrangian only reappeared in 1965 in Willmore's work who proposed the famous conjecture about minimisers of the Willmore energy for tori [47], which was finally proved in 2012 by Marques and Neves [23]. 
In higher codimension, we can also define the Willmore energy as

$$
W(\vec{\Phi})=\int_{\Sigma}|\vec{H}|^{2} d \operatorname{vol}_{g},
$$

where $\vec{H}$ is the mean curvature vector (the half-trace of the second fundamental form). It has the fundamental property of being invariant under conformal transformations (of the ambient space). In particular, since minimal surfaces $(\vec{H}=0)$ are absolute minimisers, inversions of complete minimal surfaces with finite total curvature are Willmore surfaces (though they may have branch points in general). Furthermore, Bryant showed that all immersions of the sphere in $\mathbb{R}^{3}$ are inversions of complete minimal surfaces with embedded planar ends [8].

Now, a basic problem that we can address is to try to understand the following quantities: let $\gamma \in \pi_{k}\left(\operatorname{Imm}\left(M^{2}, \mathbb{R}^{n}\right)\right)$ be a generator (of regular homotopy of immersions) and let

$$
\beta_{\gamma}=\inf _{\left\{\vec{\Phi}_{t}\right\} \simeq \gamma} \sup _{t \in S^{k}} W\left(\vec{\Phi}_{t}\right) .
$$

Then one would like to understand if we can estimate these numbers and get some information on the critical immersions realising them (if it is possible to realise the width of these min-max problems).

The first non-trivial number is given as follows: let $M^{2}=S^{2}, n=3$, and $\gamma \in$ $\pi_{1}\left(\operatorname{Imm}\left(S^{2}, \mathbb{R}^{n}\right)\right) \simeq \mathbb{Z} \times \mathbb{Z}_{2}$ be a non-trivial class (Smale [43]). Then we define

$$
\beta_{\gamma}=\inf _{\left\{\vec{\Phi}_{t}\right\} \simeq \gamma} \sup _{t \in[0,1]} W\left(\vec{\Phi}_{t}\right) .
$$

By the work of Smale, the space of immersions from the round sphere $S^{2}$ in the three-space $\mathbb{R}^{3}$ is path-connected $\left(\pi_{0}\left(\operatorname{Imm}\left(S^{2}, \mathbb{R}^{3}\right)\right)=\{0\}\right)$, we can define

$$
\beta_{0}=\inf _{\left\{\vec{\Phi}_{t}\right\} \in \Omega} \sup _{t \in[0,1]} W\left(\vec{\Phi}_{t}\right),
$$

where $\Omega$ is the set of path $\left\{\vec{\Phi}_{t}\right\}_{t \in[0,1]} \subset \operatorname{Imm}\left(S^{2}, \mathbb{R}^{3}\right)$ such that $\vec{\Phi}_{0}=\iota$ and $\vec{\Phi}_{1}=-\iota$, where $\iota: S^{2} \rightarrow \mathbb{R}^{3}$ is the standard embedding of the round sphere. These two min-max widths are equal since the Froissart-Morin eversion generates $\pi_{1}\left(\operatorname{Imm}\left(S^{2}, \mathbb{R}^{3}\right)\right.$ ) (see [36]). We will now explain what can be said about this problem in general and show a path to determine (1.1) and find which immersions may realise it. In relationship with these quantities, Kusner proposed the following conjecture.

Conjecture (Kusner, 1980's [19]) We have $\beta_{0}=16 \pi$, and an optimal path is given by a Willmore gradient flow starting from the inversion of Bryant's minimal surface with 4 embedded ends.

Thanks to Bryant's classification [29] and our extension to a large class of branched Willmore spheres [29], it makes particularly sense to compute the index of inversions of complete minimal surfaces with finite total curvature in $\mathbb{R}^{3}$. Indeed, we have the following result.

Theorem 1.1 (Rivière [36], M. [25,26]) There exists compact true branched Willmore spheres $\vec{\Phi}_{1}, \ldots, \vec{\Phi}_{p}, \vec{\Psi}_{1}, \ldots, \vec{\Psi}_{q}: S^{2} \rightarrow \mathbb{R}^{3}$ such that

$$
\beta_{0}=\sum_{i=1}^{p} W\left(\vec{\Phi}_{p}\right)+\sum_{j=1}^{q}\left(W\left(\vec{\Psi}_{j}\right)-4 \pi \theta_{j}\right)
$$


and

$$
\sum_{i=1}^{p} \operatorname{Ind}_{W}\left(\vec{\Phi}_{i}\right)+\sum_{j=1}^{q} \operatorname{Ind}_{W}\left(\vec{\Psi}_{j}\right) \leq 1
$$

where $\theta_{j}=\theta_{0}\left(\vec{\Psi}_{j}, p_{j}\right) \in \mathbb{N}$ is the multiplicity of $\vec{\Psi}_{j}$ at some point $p_{j} \in \vec{\Psi}_{j}\left(S^{2}\right)$.

Here, recall that a Willmore surface $\vec{\Phi}: \Sigma \rightarrow \mathbb{R}^{n}$ has no first residue if for all path $\gamma$ around a branch point $p$ of $\vec{\Phi}$ (which does not contain or intersect other branch points)

$$
\vec{\gamma}_{0}(\vec{\Phi}, p)=\frac{1}{4 \pi} \operatorname{Im} \int_{\gamma}\left(\partial \vec{H}+|\vec{H}|^{2} \partial \vec{\Phi}+2 g^{-1} \otimes\left\langle\vec{H}, \vec{h}_{0}\right\rangle \otimes \vec{\partial} \vec{\Phi}\right)=0 .
$$

We refer to $[2,29,34]$ for more information on this quantity.

This theorem shows that the previous conjecture should be interpreted as follows.

Conjecture Let $\vec{\Phi}_{1}, \ldots, \vec{\Phi}_{p}, \vec{\Psi}_{1}, \ldots, \vec{\Psi}_{q}$ be given by (1.2). Then $p=1, q=0$ and $\vec{\Phi}_{1}$ is the inversion of Bryant's minimal surface with 4 embedded planar ends.

\section{Main results}

If $\vec{\Psi}: \Sigma \rightarrow \mathbb{R}^{3}$ is a branched Willmore sphere, we write for all admissible variation $\vec{v} \in \operatorname{Var}(\vec{\Psi}) \subset W^{2,2}\left(\Sigma, \mathbb{R}^{3}\right)$ (see Sect. 3 for more details)

$$
Q_{\vec{\Psi}}(\vec{v})=D^{2} W(\vec{\Psi})(\vec{v}, \vec{v})
$$

the quadratic form of the second derivative of the Willmore energy $W$ at $\vec{\Psi}$. Then we define the Willmore Morse index as the maximum dimension of sub-vector spaces of $\operatorname{Var}(\vec{\Psi})$ on which $Q_{\vec{\Psi}}$ is negative definite.

Theorem A Let $\Sigma$ be a closed Riemann surface and let $\vec{\Psi}: \Sigma \rightarrow \mathbb{R}^{3}$ be a branched Willmore surface, $\vec{n}_{\vec{\Psi}}: \Sigma \rightarrow S^{2}$ be the unit normal of $\vec{\Psi}, g=\vec{\Psi}^{*} g_{\mathbb{R}^{3}}$ be the induced metric on $\Sigma$ and assume that $\vec{\Psi}$ is the inversion of a complete minimal surface $\vec{\Phi}: \Sigma \backslash\left\{p_{1}, \ldots, p_{n}\right\} \rightarrow \mathbb{R}^{3}$ with embedded ends. Assume that $0 \leq m \leq n$ is fixed such that $p_{1} \ldots, p_{m}$ are catenoid ends, while $p_{m+1}, \ldots, p_{n}$ are planar ends, and for all $1 \leq j \leq m$, let $\beta_{j}=\left|\operatorname{Flux}\left(\vec{\Phi}, p_{j}\right)\right|>0$ be the flux of $\vec{\Phi}$ at $p_{j}$. There exists a symmetric matrix $\Lambda(\overrightarrow{\vec{\Psi}}) \in \operatorname{Sym}_{n}(\mathbb{R})$ defined by

$$
\Lambda(\vec{\Psi})=\left(\begin{array}{ccccccc}
2 \beta_{1}^{2} & \lambda_{1,2} & \cdots & \cdots & \cdots & \cdots & \lambda_{1, n} \\
\lambda_{1,2} & 2 \beta_{2}^{2} & \cdots & \cdots & \cdots & \cdots & \lambda_{2, n} \\
\vdots & \ddots & \ddots & \ddots & \ddots & \ddots & \vdots \\
\lambda_{1, m} & \cdots & \cdots & 2 \beta_{m}^{2} & \cdots & \cdots & \lambda_{m, n} \\
\lambda_{1, m+1} & \cdots & \cdots & \cdots & 0 & \cdots & \lambda_{m+1, n} \\
\vdots & \ddots & \ddots & \ddots & \ddots & \ddots & \vdots \\
\lambda_{1, n} & \lambda_{2, n} & \cdots & \cdots & \cdots & \cdots & 0
\end{array}\right)
$$

with the following property: for all $\vec{v} \in \operatorname{Var}(\vec{\Psi})$ such that $v=\left\langle\vec{v}, \vec{n}_{\vec{\Psi}}\right\rangle \in C^{2}(\Sigma)$, there exists a function $v_{0} \in W^{2,2}(\Sigma)$ such that $v_{0}\left(p_{i}\right)=0$ for all $1 \leq i \leq n$, and if $u_{0}=|\vec{\Phi}|^{2} v_{0}$, we 
have the identity

$$
Q_{\vec{\Psi}}(\vec{v})=\frac{1}{2} \int_{\Sigma}\left(\Delta_{g} u_{0}-2 K_{g} u_{0}\right)^{2} d \operatorname{vol}_{g}+8 \pi \sum_{i=1}^{m} \beta_{i}^{2} v^{2}\left(p_{i}\right)+4 \pi \sum_{\substack{1 \leq i, j \leq n \\ i \neq j}} \lambda_{i, j} v\left(p_{i}\right) v\left(p_{i}\right) .
$$

Furthermore, for all $a=\left(a_{1}, \ldots, a_{n}\right) \in \mathbb{R}^{n}$, there exists an admissible variation $\vec{v} \in$ $W^{2,2} \cap W^{1, \infty}\left(\Sigma, \mathbb{R}^{3}\right)$ such that $\left(v\left(p_{1}\right), \ldots, v\left(p_{n}\right)\right)=\left(a_{1}, \ldots, a_{n}\right)\left(\right.$ where $\left.v=\left\langle\vec{v}, \vec{n}_{\vec{\Psi}}\right\rangle\right)$ and

$$
Q_{\vec{\Psi}}(\vec{v})=8 \pi \sum_{i=1}^{m} \beta_{i}^{2} v^{2}\left(p_{i}\right)+4 \pi \sum_{\substack{1 \leq i, j \leq n \\ i \neq j}} \lambda_{i, j} v\left(p_{i}\right) v\left(p_{j}\right)
$$

Therefore, we have

$$
\operatorname{Ind}_{W}(\vec{\Psi})=\operatorname{Ind} \Lambda(\vec{\Psi}) \leq n-1,
$$

where Ind $\Lambda(\vec{\Psi})$ is the number of negative eigenvalues of $\Lambda(\vec{\Psi})$.

The index of a (finite dimensional) matrix is defined as usual by the number of (strictly) negative eigenvalues.

Remark This theorem was first presented in detail on November 13, 2018 at the Institute for Advanced Study in the seminar Variational Methods in Geometry Seminar

https://www.math.ias.edu/seminars/abstract?event=138881

The video was uploaded and is freely available on the internet since then at the following links:

https://video.ias.edu/varimethodsgeo/2018/1113-AlexisMichelat

https://www.youtube.com/watch?v=1AYcy22OIec

The interested reader will find at 1:05 the main theorem, at 1:31 and 1:35 the special negative variations with logarithm behaviour at the ends and at 1:39 the additional term coming out for variations including a logarithm term.

Remark There are examples of complete minimal surfaces of genus 1 with planar ends discovered by Costa and Shamaev [10,41]. Kusner and Schmitt also studied the moduli space of such minimal surfaces in detail (see [20]), and showed in particular that there are no examples with three planar ends (this is the first non-trivial case thanks to Schoen's theorem on the characterisation of the catenoid as the only complete minimal surface with 2 embedded ends [39]). They all have an even number of ends (at least 4). In fact, all values of ends $2 n \geq 4$ are attained.

Corollary B Let $\vec{\Phi}: S^{2} \rightarrow \mathbb{R}^{3}$ be a Willmore immersion. Then

$$
\operatorname{Ind}_{W}(\vec{\Phi}) \leq \frac{1}{4 \pi} W(\vec{\Phi})-1 .
$$

In general, we can obtain a general bound which generalises the main result of [28] to the case of branched Willmore surfaces.

Theorem C Let $\vec{\Psi}: \Sigma \rightarrow \mathbb{R}^{3}$ be a branched Willmore surface and assume that $\vec{\Psi}$ is the inversion of a complete minimal surface with finite total curvature $\vec{\Phi}: \Sigma \backslash\left\{p_{1}, \ldots, p_{n}\right\} \rightarrow$ $\mathbb{R}^{3}$. Then there exists a universal symmetric matrix $\Lambda=\Lambda(\vec{\Psi})=\left\{\lambda_{i, j}\right\}_{1 \leq i, j \leq n}$ such that 
for all $\vec{v} \in \operatorname{Var}(\vec{\Psi}) \cap C^{\infty}\left(\Sigma, \mathbb{R}^{3}\right)$, if $v=\left\langle\vec{v}, \vec{n}_{\vec{\Psi}}\right\rangle$, then there exists a function $v_{0} \in W^{2,2}(\Sigma)$ such that $v_{0}\left(p_{i}\right)=0$ for all $1 \leq i \leq n$, and if $u_{0}=|\vec{\Phi}|^{2} v_{0}$, we have the identity

$$
Q_{\vec{\Psi}}(\vec{v})=\frac{1}{2} \int_{\Sigma}\left(\Delta_{g} u_{0}-2 K_{g} u_{0}\right)^{2} d \operatorname{vol}_{g}+4 \pi \sum_{1 \leq i, j \leq n} \lambda_{i, j} v\left(p_{i}\right) v\left(p_{j}\right),
$$

Furthermore, there exists an admissible variation $\vec{v} \in \operatorname{Var}(\vec{\Psi})$ such that

$$
Q_{\vec{\Psi}}(\vec{v})=4 \pi \sum_{1 \leq i, j \leq n} \lambda_{i, j} v\left(p_{i}\right) v\left(p_{j}\right)
$$

In particular, we have

$$
\operatorname{Ind}_{W}(\vec{\Psi})=\operatorname{Ind} \Lambda(\vec{\Psi}) \leq n=\frac{1}{4 \pi} W(\vec{\Psi})-\frac{1}{2 \pi} \int_{\Sigma} K_{g} d \operatorname{vol}_{g}+\chi(\Sigma) .
$$

Remark For true branched immersions with ends of multiplicity at most 2, we may get the bound

$$
\operatorname{Ind}_{W}(\vec{\Psi}) \leq n-1=\frac{1}{4 \pi} W(\vec{\Psi})-\frac{1}{2 \pi} \int_{\Sigma} K_{g} d \operatorname{vol}_{g}+\chi(\Sigma)-1 .
$$

by showing that $\lambda_{i, i}=0$ for ends of multiplicity 2 . Since the proof is just a lengthy but straightforward computation, it is omitted (see [27], pp. 355-367).

Added in proof. Recently Jonas Hirsch and Elena Mäder-Baumdicker wrote a paper on this subject in the special case of minimal surfaces with flat ends [17].

\section{The second derivative of the Willmore energy as a renormalised energy}

Let $\Sigma$ be a closed Riemann surface, $n \in \mathbb{N}, p_{1}, \ldots, p_{n} \in \Sigma$ be fixed distinct points and $\vec{\Phi}: \Sigma \backslash\left\{p_{1}, \ldots, p_{n}\right\} \rightarrow \mathbb{R}^{3}$ be a complete minimal surface with finite total curvature and assume without loss of generality that $0 \notin \vec{\Phi}\left(\Sigma \backslash\left\{p_{1}, \ldots, p_{n}\right\}\right) \subset \mathbb{R}^{3}$. Then the inversion

$$
\vec{\Psi}=\frac{\vec{\Phi}}{|\vec{\Phi}|^{2}}: \Sigma \rightarrow \mathbb{R}^{3}
$$

is a compact branched Willmore surface. Now, recall that we defined in [26] a notion of admissible variations of the Willmore energy as the maximum set of variations for which the second derivative of the Willmore energy is well-defined.

Theorem 3.1 [26] Let $\Sigma$ be a closed Riemann surface and let $\vec{\Psi}: \Sigma \rightarrow \mathbb{R}^{d}$ be a branched Willmore immersion and let $g=\vec{\Psi}^{*} g_{\mathbb{R}^{d}}$ be the induced metric. Then the second derivative $D^{2} W(\vec{\Psi})$ is well-defined at some point

$$
\vec{w}=\mathscr{E}_{\vec{\Psi}}\left(\Sigma, \mathbb{R}^{d}\right)=W^{2,2} \cap W^{1, \infty}\left(\mathbb{R}^{d}\right) \cap\left\{\vec{w}: \vec{w}(p) \in T_{\vec{\Psi}(p)} \mathbb{R}^{d} \text { for all } p \in \Sigma\right\}
$$

if and only if

$$
d \vec{w} \in L^{\infty}\left(\Sigma, g_{0}\right) \quad \text { and } \mathscr{L}_{g}^{\perp} \vec{w} \in L^{2}\left(\Sigma, d \operatorname{vol}_{g}\right),
$$

where $g_{0}$ is any fixed smooth metric on $\Sigma$, and $\mathscr{L}_{g}^{\perp}=\Delta_{g}^{\perp}+\mathscr{A}(\cdot)$ is the Jacobi operator and $\mathscr{A}$ is the Simons operator. We denote by $\operatorname{Var}(\vec{\Psi})$ this space of admissible variations. 
Notice that at a branch point of multiplicity $m \geq 1$, the condition are equivalent to

$$
\frac{|\nabla \vec{w}|}{|z|^{m-1}} \in L^{\infty}\left(D^{2}\right), \quad \text { and } \frac{(\Delta \vec{w})^{\perp}}{|z|^{m-1}} \in L^{2}\left(D^{2}\right) .
$$

In particular, if $\vec{w}$ is a smooth variation, the conditions are equivalent to

$$
\vec{w}=\vec{w}(0)+\operatorname{Re}\left(\vec{\gamma} z^{m}\right)+O\left(|z|^{m+1}\right) .
$$

We can now define the Willmore Morse index as follows (see [26]).

Definition 3.2 Let $\Sigma$ be a closed Riemann surface and let $\vec{\Phi}: \Sigma \rightarrow \mathbb{R}^{n}$ be a branched Willmore immersion. Then Willmore index of $\vec{\Phi}$, denoted by $\operatorname{Ind}_{W}(\vec{\Phi})$, is equal to the dimension of the maximal sub-vector space $V \subset \operatorname{Var}(\vec{\Psi})$ on which the quadratic form second variation $Q_{\vec{\Psi}}(\cdot)=D^{2} W(\vec{\Phi})(\cdot, \cdot)$ is negative definite.

Now, thanks to Proposition 4.5 of [28], assuming that $\vec{\Psi}$ is smooth, for all $\vec{v}=v \vec{n}_{\vec{\Psi}} \in$ $\operatorname{Var}(\vec{\Psi})$, we have

$$
\begin{aligned}
Q_{\vec{\Psi}}(\vec{v})=D^{2} W(\vec{\Psi})(\vec{v}, \vec{v})= & \int_{\Sigma}\left\{\frac{1}{2}\left(\Delta_{g} u-2 K_{g} u\right)^{2} d \operatorname{vol}_{g}\right. \\
& \left.-d\left(\left(\Delta_{g} u+2 K_{g} u\right) * d u-\frac{1}{2} * d|d u|_{g}^{2}\right)\right\} .
\end{aligned}
$$

where $u=|\vec{\Phi}|^{2} v$. However, conformally minimal branched Willmore spheres are generally not smooth, so this formula needs to be generalised in two directions. First, one needs to consider vectorial variations, and secondly the second variation $\left.\frac{d^{2}}{d t^{2}} \vec{\Phi}_{t}\right|_{\mid t=0}$ will have to be taken into account. As the derivation is particularly long, we differ it to the appendix (see Theorem 10.5).

Theorem 3.3 Let $\vec{\Psi}: \Sigma \rightarrow \mathbb{R}^{3}$ be a branched Willmore sphere, and assume that $\vec{\Psi}$ is the inversion of a complete minimal surface $\vec{\Phi}: \Sigma \backslash\left\{p_{1}, \ldots, p_{n}\right\} \rightarrow \mathbb{R}^{3}$ with finite total curvature, and let $\vec{v}$ be an admissible variation. Make the decomposition $\vec{v}=-v \vec{n}_{\vec{\Psi}}+$ $2 \operatorname{Re}(\alpha \otimes \partial \vec{\Psi})$. If $u=|\vec{\Phi}|^{2} v$, we have

$$
\begin{aligned}
& D^{2} \mathscr{W}(\vec{\Psi})(\vec{v}, \vec{v})=\int_{\Sigma}\left(\frac{1}{2}\left(\mathscr{L}_{g} u\right)^{2} d \operatorname{vol}_{g}\right. \\
& \quad-d \operatorname{Im}\left(\left(\Delta_{g} u+2 K_{g} u+4 \operatorname{Re}\left(g^{-1} \otimes h_{0} \otimes \bar{\partial} \alpha\right)\right)\left(2 \partial u+h_{0} \otimes \alpha\right)\right. \\
& \quad-\partial\left|2 \partial u+h_{0} \otimes \alpha\right|_{g}^{2}+2 g^{-1} \otimes \bar{\partial}(g \otimes \bar{\alpha}) \otimes\left(2 g^{-1} \otimes h_{0} \otimes \bar{\partial} u-K_{g} g \otimes \bar{\alpha}\right) \\
& \quad+4\left\langle\vec{\Phi}, \vec{n}_{\vec{\Phi}}\right\rangle g^{-1} \otimes h_{0} \otimes \bar{\partial}\left(|\vec{\Phi}|^{2} v^{2}+\frac{1}{|\vec{\Phi}|^{2}} g \otimes|\alpha|^{2}\right) \\
& \quad-8 g^{-1} \otimes h_{0} \otimes \bar{\partial}\left(|\vec{\Phi}|^{2}\left\langle\vec{\Phi}, \vec{n}_{\vec{\Phi}}\right\rangle v^{2}+2 \operatorname{Re}(\alpha \otimes \partial \log |\vec{\Phi}|)|\vec{\Phi}|^{2} v\right) \\
& \left.\left.\quad+4 K_{g}\left(\left\langle\vec{\Phi}, \vec{n}_{\vec{\Phi}}\right\rangle v+2 \operatorname{Re}(\alpha \otimes \partial \log |\vec{\Phi}|)\right) g \otimes \bar{\alpha}\right)\right) \\
& =\int_{\Sigma}\left(\frac{1}{2}\left(\mathscr{L}_{g} u\right)^{2} d \operatorname{vol}_{g}-d \omega(u, \alpha)\right),
\end{aligned}
$$

where $g=\vec{\Phi}^{*} g_{\mathbb{R}^{3}}$, and $\mathscr{L}_{g}=\Delta_{g}-2 K_{g}$ is the Jacobi operator of $\vec{\Phi}$. 
Remark 3.4 1. We can see directly that the formula reduces to the previous one if $\vec{\Psi}$ is smooth and $\vec{v}=-v \vec{n}_{\vec{\Psi}}$ is normal. Indeed, by the proof of Theorem 4.1, we have at a branch point in any complex chart centred at $z=0 h_{0}=O\left(\frac{1}{|z|}\right)$, while up to scaling

$$
|\vec{\Phi}|^{2}=\frac{1}{m^{2}} \frac{1}{|z|^{2 m}}(1+O(|z|)) \quad \text { and } \quad e^{2 \lambda}=\frac{1}{|z|^{2 m+2}}(1+O(|z|)) .
$$

Furthermore, since $\vec{\Psi}$ is smooth, we deduce by the regularity criterion of Bernard-Rivière [1] that $H_{\vec{\Psi}}=-2\left\langle\vec{n}_{\vec{\Phi}}, \vec{\Phi}\right\rangle \in L^{\infty}(\Sigma)$ (by Lemma 10.8 of the appendix) and we directly get

$$
\begin{aligned}
4\left\langle\vec{\Phi}, \vec{n}_{\vec{\Phi}}\right\rangle g^{-1} \otimes h_{0} \otimes \bar{\partial}\left(|\vec{\Phi}|^{2} v^{2}\right)= & O(1) \times O\left(|z|^{-(2 m+2)}\right) \times O\left(|z|^{-1}\right) \\
& \times O\left(|z|^{-(2 m+1)}\right)=O(1)
\end{aligned}
$$

and the other two additional terms are estimated similarly, so that one can apply Stokes theorem and neglect those components.

2. By [1], the mean curvature $H_{\vec{\Psi}}$ is in particular Lipschitz, which implies in that $H_{\vec{\Psi}}=$ $c_{0}+O(|z|)$ fo some $c_{0} \in \mathbb{R}$. Furthermore, we have by Lemma 10.8

$$
h_{\vec{\Psi}}^{0}=-\frac{1}{|\vec{\Phi}|^{2}} h_{\vec{\Phi}}^{0}=O\left(|z|^{2 m-1}\right)
$$

Therefore, we deduce that have $\partial_{z} \vec{n}=-H_{\vec{\Psi}} \partial_{z} \vec{\Psi}-e^{-2 \mu} h_{\vec{\Psi}}^{0} \partial_{\bar{z}} \vec{\Psi}=-c_{0} \vec{A}_{0} z^{m-1}+O\left(|z|^{m}\right)$ for some $\vec{A}_{0} \in \mathbb{C}^{3} \backslash\{0\}$. Integrating this equation and using the fact that $\vec{n}_{\vec{\Psi}}$ is real, we deduce that

$$
\vec{n}_{\vec{\Psi}}=\vec{n}_{\vec{\Psi}}\left(p_{i}\right)-\frac{2 c_{0}}{m} \operatorname{Re}\left(\vec{A}_{0} z^{m}\right)+O\left(|z|^{m+1}\right) .
$$

Therefore, if $\vec{\Psi}$ is smooth, for all real-valued function $v \in W^{2,2}(\Sigma)$ such that $|d v|_{g} \in$ $L^{\infty}(\Sigma)$ and $\Delta_{g} v \in L^{2}\left(\Sigma, d \operatorname{vol}_{g}\right)$, the variation $v \vec{n}_{\vec{\Psi}}$ is admissible.

Now, thanks to the Stokes theorem applied to (3.1), we have

$$
Q_{\vec{\Psi}}(\vec{v})=\lim _{\varepsilon \rightarrow 0}\left(\frac{1}{2} \int_{\Sigma_{\varepsilon}}\left(\Delta_{g} u-2 K_{g} u\right)^{2} d \operatorname{vol}_{g}+\sum_{i=1}^{n} \int_{\partial B_{\varepsilon}\left(p_{i}\right)} \omega(u, \alpha)\right),
$$

where

$$
\Sigma_{\varepsilon}=\Sigma \backslash \bigcup_{i=1}^{n} \bar{B}_{\varepsilon}\left(p_{i}\right)
$$

In particular, the limit (3.2) exists for all such $\vec{v} \in \operatorname{Var}(\vec{\Psi})$. Here, the balls $\bar{B}_{\varepsilon}\left(p_{i}\right)$ are fixed after the following definition for some covering $\left(U_{1}, \ldots, U_{n}\right)$ of $\left\{p_{1}, \ldots, p_{n}\right\}$ fixed once and for all.

Definition 3.5 We say that a family of chart domains $\left(U_{1}, \ldots, U_{n}\right)$ is a covering of $\left\{p_{1}, \ldots, p_{n}\right\} \subset \Sigma$ if $p_{i} \in U_{i}$ for all $1 \leq i \leq n$ and $U_{i} \cap U_{j}=\varnothing$ for all $1 \leq i \leq n$. For all $1 \leq i \leq n$ if $\varphi_{i}: U_{i} \rightarrow B_{\mathbb{C}}(0,1) \subset \mathbb{C}$ is a complex chart such that $\varphi_{i}\left(p_{i}\right)=0$ and $\varphi_{i}\left(U_{i}\right)=B_{\mathbb{C}}(0,1)$, we define for all $0<\varepsilon<1$

$$
B_{\varepsilon}\left(p_{i}\right)=\varphi_{i}^{-1}\left(B_{\mathbb{C}}(0, \varepsilon)\right) .
$$


This definition is independent of the chart $\varphi_{i}: U_{i} \rightarrow B_{\mathbb{C}}(0,1) \subset \mathbb{C}$ such that $\varphi_{i}\left(U_{i}\right)=$ $B_{\mathbb{C}}(0,1)$ and $\varphi_{i}\left(p_{i}\right)=0$.

The independence of the chart $\varphi_{i}$ with the above properties is a trivial consequence of Schwarz lemma (see [28] for more details).

Let us now say a few words concerning the analysis of this article. By a standard method due to Smale, we construct for all $\varepsilon>0$ small enough $n$ real-valued functions $u_{\varepsilon}^{1}, \ldots, u_{\varepsilon}^{n}$, such that for all $1 \leq i \leq n$, the function $u_{\varepsilon}^{i}: \Sigma_{\varepsilon}^{i}=\Sigma \backslash\left(\bar{B}_{\varepsilon}\left(p_{i}\right) \cup\left\{p_{1}, \ldots, p_{n}\right\}\right) \rightarrow \mathbb{R}$ satisfies the system

$$
\left\{\begin{aligned}
\mathscr{L}_{g}^{2} u_{\varepsilon}^{i} & =0 & & \text { in } \Sigma_{\varepsilon}^{i} \\
u_{\varepsilon}^{i} & =u & & \text { on } \partial \Sigma_{\varepsilon}^{i} \\
\partial_{\nu} u_{\varepsilon}^{i} & =\partial_{\nu} u & & \text { on } \partial \Sigma_{\varepsilon}^{i} .
\end{aligned}\right.
$$

If $u_{\varepsilon}=u-\sum_{i=1}^{n} u_{\varepsilon}^{i}$, this allows us to perform an expansion and an integration by parts

$$
\begin{aligned}
& \frac{1}{2} \int_{\Sigma_{\varepsilon}}\left(\mathscr{L}_{g} u\right)^{2} d \operatorname{vol}_{g}=\frac{1}{2} \int_{\Sigma_{\varepsilon}}\left(\mathscr{L}_{g} u_{\varepsilon}\right)^{2} d \operatorname{vol}_{g}+\frac{1}{2} \int_{\Sigma_{\varepsilon}}\left(\mathscr{L}_{g} u_{\varepsilon}^{i}\right)^{2} d \operatorname{vol}_{g}+\sum_{i=1}^{n} \int_{\Sigma_{\varepsilon}} \mathscr{L}_{g} u_{\varepsilon} \mathscr{L}_{g} u_{\varepsilon}^{i} d \operatorname{vol}_{g} \\
& \quad+\sum_{1 \leq i \neq j \leq n} \frac{1}{2} \int_{\Sigma_{\varepsilon}} \mathscr{L}_{g} u_{\varepsilon}^{i} \mathscr{L}_{g} u_{\varepsilon}^{j} d \operatorname{vol}_{g}=\frac{1}{2} \int_{\Sigma_{\varepsilon}}\left(\mathscr{L}_{g} u_{\varepsilon}\right)^{2} d \operatorname{vol}_{g}+\sum_{i=1}^{n} \int_{\Sigma_{\varepsilon}} \mathscr{L}_{g} u_{\varepsilon} \mathscr{L}_{g} u_{\varepsilon}^{i} d \operatorname{vol}_{g} \\
& \quad+\sum_{1 \leq i \neq j \leq n} \int_{\Sigma_{\varepsilon}} \frac{1}{2} \mathscr{L}_{g} u_{\varepsilon}^{i} \mathscr{L}_{g} u_{\varepsilon}^{j} d \operatorname{vol}_{g}+\sum_{i, j=1}^{n} \frac{1}{2} \int_{\partial B_{\varepsilon}}\left(u_{\varepsilon}^{i} \partial_{\nu}\left(\mathscr{L}_{g} u_{\varepsilon}^{i}\right)-\partial_{\nu}\left(u_{\varepsilon}^{i}\right) \mathscr{L}_{g} u_{\varepsilon}^{i}\right) d \mathscr{H}^{1} .
\end{aligned}
$$

Then, we prove in Sect. 6 by an indicial root analysis (Sect. 5) that

$$
\sum_{i, j=1}^{n} \frac{1}{2} \int_{\partial B_{\varepsilon}\left(p_{j}\right)}\left(u_{\varepsilon}^{i} \partial_{\nu}\left(\mathscr{L}_{g} u_{\varepsilon}^{i}\right)-\partial_{\nu}\left(u_{\varepsilon}^{i}\right) \mathscr{L}_{g} u_{\varepsilon}^{i}\right) d \mathscr{H}^{1}=-\sum_{i=1}^{n} \int_{\partial B_{\varepsilon}\left(p_{i}\right)} \omega(u, \alpha)+O(1)
$$

and the remaining terms are all bounded. Finally, refining this estimate, we show that $u_{\varepsilon} \underset{\varepsilon \rightarrow 0}{\longrightarrow}$ $u_{0}$ and that there exists $\lambda_{i, j} \in \mathbb{R}$ such that

$$
Q_{\vec{\Psi}}(\vec{v})=\frac{1}{2} \int_{\Sigma}\left(\mathscr{L}_{g} u_{0}\right)^{2} d \operatorname{vol}_{g}+4 \pi \sum_{i, j=1}^{n} \lambda_{i, j} v\left(p_{i}\right) v\left(p_{j}\right)
$$

This is done in Theorem 6.6 in the case of embedded ends and in Theorem 8.1. This immediately implies the upper estimate (already proven in Theorem 5.1). Then, using the functions $v_{0}=|\vec{\Phi}|^{-2} u_{0}$ as test functions, we obtain the equality for the Morse index (Theorems 7.1 and 8.3).

\section{Explicit description of the admissible variations}

Before delving into the proof of the main theorem, we need to describe the set of admissible variations more precisely. First, let us examine an example.

Let $\vec{\Phi}: \Sigma \backslash\left\{p_{1}, \ldots, p_{n}\right\} \rightarrow \mathbb{R}^{3}$ and assume that $p_{i}$ is an embedded end of catenoid growth. Up to a rotation, we have $\vec{n}_{\vec{\Psi}}\left(p_{i}\right)=(0,0,1)$, we will see that we have (see the proof of Theorem 6.2) for some $\beta_{i} \neq 0$ an expansion of the form

$$
\vec{\Psi}(z)=\operatorname{Re}\left(\vec{A}_{0} z\right)+\beta_{i}|z|^{2} \log |z| \vec{e}_{3}+O\left(|z|^{3} \log ^{2}|z|\right)
$$




$$
\vec{n}_{\vec{\Psi}}=\vec{e}_{3}+\operatorname{Re}\left(\left(-2 \beta_{i} z \log |z|-\beta_{i} z\right) \vec{A}_{0}\right)+O\left(|z|^{2} \log ^{2}|z|\right)
$$

where $\vec{A}_{0}=(1,-i, 0)$, and $\vec{e}_{3}=(0,0,1)$. In particular, if $v \in C^{\infty}(\Sigma)$, and $v\left(p_{i}\right) \neq 0$, the function $\vec{\Psi}_{t}=\vec{\Psi}+t v \vec{n}_{\vec{\Psi}}$ admits the expansion

$$
\vec{\Psi}_{t}=\operatorname{Re}\left(\vec{A}_{0}\left(z\left(1-t \beta_{i} v\left(p_{i}\right)-2 t \beta_{i} v\left(p_{i}\right) \log |z|\right)\right)\right)+t v \vec{e}_{3}+O\left(|z|^{2} \log ^{2}|z|\right) .
$$

Therefore, for all $t \neq 0$, we have

$$
\left|\partial_{x_{1}} \vec{\Phi}_{t} \times \partial_{x_{2}} \vec{\Phi}_{t}\right|=4 t^{2} \beta_{i}^{2} v^{2}\left(p_{i}\right) \log ^{2}|z|\left(1+O\left(\frac{1}{\log |z|}\right)\right) \notin L^{\infty}(\mathbb{D})
$$

so $\vec{\Psi}_{t}$ is not a weak immersion [35].

If $\vec{\Psi}$ has a branch point of higher order $m \geq 2$, then we will see that $\vec{\Psi}_{t}=\vec{\Psi}+t \vec{n}_{\vec{\Psi}}$ is not admissible in general. Choosing a conformal parametrisation of $\vec{\Psi}$ from the unit disk $\mathbb{D} \rightarrow \Sigma$ at a branch point we have for all $i=1,2$ (using $\mathbb{Z}_{2}$ indices)

$$
\partial_{x_{i}} \vec{\Psi}_{t}=\left(1-t e^{-2 \lambda} \mathbb{I}_{i, i}\right) \partial_{x_{i}} \vec{\Psi}-t e^{-2 \lambda} \mathbb{I}_{1,2} \partial_{x_{i+1}} \vec{\Psi}
$$

where $\mathbb{I}$ is the second fundamental form. Therefore, we have

$$
\begin{aligned}
\partial_{x_{1}} \vec{\Psi}_{t} \times \partial_{x_{2}} \vec{\Psi}_{t} & =\left(\left(1-t e^{-2 \lambda} \mathbb{I}_{1,1}\right)\left(1-t e^{-2 \lambda} \mathbb{I}_{2,2}\right)-t^{2} e^{-4 \lambda} \mathbb{I}_{1,2}^{2}\right) \partial_{x_{1}} \vec{\Psi} \times \partial_{x_{2}} \vec{\Psi} \\
& =\left(1-t e^{-2 \lambda}\left(\mathbb{I}_{1,1}+\mathbb{I}_{2,2}\right)+t^{2} e^{-4 \lambda}\left(\mathbb{I}_{1,1} \mathbb{I}_{2,2}-\mathbb{I}_{1,2}^{2}\right)\right) \partial_{x_{1}} \vec{\Psi} \times \partial_{x_{2}} \vec{\Psi} \\
& =\left(1-2 t H+t^{2} K\right) \partial_{x_{1}} \vec{\Psi} \times \partial_{x_{2}} \vec{\Psi}
\end{aligned}
$$

where $H$ is the mean curvature and $K$ is the Gauss curvature. Up to scaling, we deduce that

$$
\left|\partial_{x_{1}} \vec{\Psi}_{t} \times \partial_{x_{2}} \vec{\Psi}_{t}\right|=\left|1-2 t H+t^{2} K\right||z|^{2 m-2}(1+O(|z|)) .
$$

Now, assume that $\vec{\Psi}$ is not smooth at $p_{i}$, then the Bernard-Rivière criterion implies that $H \notin L^{\infty}(\mathbb{D})$ and that the variation is not admissible since the order of the branch point is not preserved.

The question is therefore to determine for a branched immersion $\vec{\Psi}: \Sigma \rightarrow \mathbb{R}^{3}$ for which $v \in W^{2,2}(\Sigma)$, there exists a $(-1,0)$-form $\alpha$ (see [13] for the analogous of Beltrami coefficients, or $(-1,1)$ forms) such that $\vec{v}=-v \vec{n}_{\vec{\Psi}}+2 \operatorname{Re}(\alpha \otimes \partial \vec{\Psi})$ is an admissible variation. The following theorem gives an algorithm to get the Taylor expansion of $v$ and $\alpha$ up to order $m$.

Theorem 4.1 Let $\vec{\Psi}: \Sigma \rightarrow \mathbb{R}^{3}$ be a branched Willmore surface which is the inversion of a complete minimal surface $\vec{\Phi}: \Sigma \backslash\left\{p_{1}, \ldots, p_{n}\right\} \rightarrow \mathbb{R}^{3}$ with finite total curvature. Let $1 \leq i \leq n$, assume that $p_{i}$ is of multiplicity $m \geq 1$, and fix a complex chart $\varphi_{i}: B_{\mathbb{C}}(0,1) \rightarrow \Sigma$ such that $\varphi_{i}(0)=p_{i}$. If $m=1$ and $\vec{\Phi}$ admits a catenoid end at $p_{i}$, then there exits $\beta_{i} \in \mathbb{R} \backslash\{0\}$ such that

$$
h_{\vec{\Phi}}^{0}=-\beta_{i} \frac{d z^{2}}{z^{2}}+O\left(\frac{1}{z}\right)
$$

and for all variation $v \in W^{2,2}(\Sigma) \cap W^{1, \infty}(\Sigma)$, the variation $\vec{v}=-v \vec{n}_{\vec{\Psi}}+2 \operatorname{Re}(\alpha \otimes \partial \vec{\Psi})$ is admissible if and only if

$$
\alpha=-2 \beta_{i} v\left(p_{i}\right) z \log |z|+\gamma_{1} z+\gamma_{2} \bar{z}+\beta,
$$

where $\beta \in W^{2,2}(\Sigma) \cap W^{1, \infty}(\Sigma)$ and $\bar{z} \log |z| \beta \in W^{2,2}(\Sigma) \cap W^{1, \infty}(\Sigma)$. 
If $m \geq 2$, for all $v \in W^{2,2}(\Sigma)$, define the vectorial variation $\vec{v}=-v \vec{n}_{\vec{\Psi}}+2 \operatorname{Re}(\alpha \otimes \partial \vec{\Psi})$ for some $(-1,0)$-form. Now assume that that $\Delta_{g} v=O\left(|A|^{2} v\right)$, and that $v$ admits a Taylor expansion of the form

$$
v=v\left(p_{i}\right)+\sum_{\substack{i, j \in \mathbb{Z} \\ 1 \leq i+j \leq m}} \sum_{k \geq 0} \operatorname{Re}\left(\gamma_{i, j}^{k} z^{i} \bar{z}^{j} \log ^{k}|z|\right)+O\left(|z|^{m+1} \log ^{N}|z|\right)
$$

for some $N \in \mathbb{N}$ (and $\gamma_{i, j}^{k} \in \mathbb{C}$ ), then $\vec{v}$ is admissible if and only if $v$ and $\alpha$ solve for some $c_{1}, c_{2}, c_{3} \in \mathbb{C}$ and $N_{1}, N_{2}, N_{3} \in \mathbb{N}$ the system

$$
\left\{\begin{aligned}
\partial v & =\frac{1}{2} h_{0} \otimes \alpha+\frac{1}{2} H g \otimes \bar{\alpha}+c_{1} z^{m-1}+O\left(|z|^{m} \log ^{N_{1}}|z|\right) \\
\partial(g \otimes \alpha) & =-g H v+c_{2}|z|^{2 m-2}+O\left(|z|^{2 m-2} \log ^{N_{2}}|z|\right) \\
\bar{\partial} \alpha & =-\left(g^{-1} \otimes \overline{h_{0}}\right) v+c_{3}\left(\frac{\bar{z}}{z}\right)^{m-1}+O\left(|z| \log ^{N_{3}}|z|\right) .
\end{aligned}\right.
$$

Now, assume that $r_{2}\left(\vec{\Psi}, p_{i}\right)=d-1 \geq 1$, i.e. that there exists $c_{0} \in \mathbb{C} \backslash\{0\}$ such that

$$
H=\operatorname{Re}\left(\frac{c_{0}}{z^{d-1}}\right)+O\left(|z|^{2-d}\right) .
$$

Then there exists $\alpha_{i, j}^{k}, \beta_{i, j}^{k} \in \mathbb{C}(i, j \in \mathbb{Z}, k \in \mathbb{N})$ almost all zero such that $v$ and $\alpha$ admit the following Taylor expansions

$$
\begin{aligned}
v= & v\left(p_{i}\right)-\frac{1}{8}\left(\frac{2 m^{2}}{(m+1-d)(m+2-2 d)} \operatorname{Re}\left(c_{0}^{2} v\left(p_{i}\right) z^{m+2-2 d} \bar{z}^{m}\right)\right. \\
& \left.+\frac{2 m^{2}-(d-1)^{2}}{(m+1-d)^{2}}\left|c_{0}\right|^{2} v\left(p_{i}\right)|z|^{2 m+2-2 d}\right) \\
& +\sum_{i+j=2 m+2-2 d+1} \sum_{k \geq 0} \operatorname{Re}\left(\alpha_{i, j}^{k} v\left(p_{i}\right) z^{i} \bar{z}^{j} \log ^{k}|z|\right) \\
& +\sum_{i+j=m, i j \neq 0} \sum_{k \geq 0} \operatorname{Re}\left(\alpha_{i, j}^{k} v\left(p_{i}\right) z^{i} \bar{z}^{j} \log ^{k}|z|\right) \\
& +\operatorname{Re}\left(\gamma_{0} z^{m}\right)+O\left(|z|^{m+1} \log ^{N}|z|\right) \\
\alpha= & -\frac{1}{2}\left(\frac{c_{0}}{m+1-d} \frac{1}{z^{d-2}} v\left(p_{i}\right)+\frac{c_{0}}{m} \frac{z}{\bar{z}^{d-1}} v\left(p_{i}\right)\right) \\
& +\sum_{3-d \leq i+j \leq 0, k \geq 0} \beta_{i, j}^{k} v\left(p_{i}\right) z^{i} \bar{z}^{j} \log ^{k}|z|+O\left(|z| \log ^{N}|z|\right) .
\end{aligned}
$$

In particular, if $2 m+2-2 d \geq m+1$, i.e. $d \leq \frac{m+1}{2}$, there are no conditions to impose. Finally, if $d=1$ and $\vec{\gamma}_{0}\left(\vec{\Psi}, p_{i}\right) \neq 0$, then there are no conditions to impose and we can take $\alpha=0$.

Proof At a branch point of multiplicity $m \geq 1$ in the smooth case the conditions on $\vec{v}$ imply that $v$ admits an expansion of the form

$$
\vec{v}=\vec{v}\left(p_{i}\right)+\operatorname{Re}\left(\vec{\gamma}_{0} z^{m}\right)+O\left(|z|^{m+1}\right) .
$$


In particular, we have

$$
\partial \vec{v} \in \operatorname{Span}_{\mathbb{C}^{3}}\left(z^{m-1}\right)+O\left(|z|^{m}\right) .
$$

We compute

$$
\begin{aligned}
\partial_{z} \vec{v}= & -\partial_{z} v \vec{n}_{\vec{\Psi}}+v\left(H \partial_{z} \vec{\Psi}+e^{-2 \lambda} h_{0} \partial_{z} \vec{\Psi}\right)+\partial_{z} \alpha \partial_{z} \vec{\Psi} \\
& +\alpha\left(\frac{1}{2} \vec{h}_{0}+2\left(\partial_{z} \mu\right) \partial_{z} \vec{\Psi}\right)+\partial_{z} \bar{\alpha} \partial_{\bar{z}} \vec{\Psi}+\frac{1}{2} \bar{\alpha} e^{2 \lambda} \vec{H} \\
= & \left(-\partial_{z} v+\frac{1}{2} h_{0} \alpha+\frac{1}{2} e^{2 \lambda} H \bar{\alpha}\right) \vec{n}_{\vec{\Psi}}+\left(\partial_{z} \alpha+2\left(\partial_{z} \lambda\right) \alpha+H v\right) \partial_{z} \vec{\Psi} \\
& +\left(\partial_{z} \bar{\alpha}+e^{-2 \lambda} h_{0} v\right) \partial_{\bar{z}} \vec{\Psi} .
\end{aligned}
$$

Since $\vec{n}$ is Lipschitz, we have for some $\vec{n}\left(p_{i}\right) \in S^{2}$ the expansion $\vec{n}=\vec{n}\left(p_{i}\right)+O(|z|)$, while for some $\vec{A}_{0} \in \mathbb{C}^{3} \backslash\{0\}$, we have $\partial_{z} \vec{\Psi}=\vec{A}_{0} z^{m-1}+O\left(|z|^{m} \log |z|\right)$. In particular, we deduce that $v$ and $\alpha$ must satisfy for some constants $c_{1}, c_{2}, c_{3}, c_{4} \in \mathbb{C}$ the system

$$
\left\{\begin{aligned}
\partial v & =\frac{1}{2} h_{0} \otimes \alpha+\frac{1}{2} H g \otimes \bar{\alpha}+c_{1} z^{m-1}+O\left(|z|^{m}\right) \\
\partial(g \otimes \alpha) & =-g H v+c_{2}|z|^{2 m-2}+O\left(|z|^{2 m-1}\right) \\
\bar{\partial} \alpha & =-\left(g^{-1} \otimes \overline{h_{0}}\right) v+c_{3}\left(\frac{\bar{z}}{\bar{z}}\right)^{m-1}+O(|z|) .
\end{aligned}\right.
$$

Therefore, thanks to the Taylor expansion of $g, H$ and $h_{0}$ given by [1], we can solve this system by induction to find the admissible functions $v$. Indeed, we first make the expansion $v=v\left(p_{i}\right)+O(|z|)$ to solve the last two equations at order 1 , which uniquely determines the first order of the Taylor expansion of $\alpha$. Then, using the first equation, we obtain the next order expansion of $v$ that we can plug again in the second and third equation until all equations are satisfied (then, the higher order terms of $v$ are free). Let us check one explicit example. At a catenoid end, we have by the forthcoming proof of Theorem 6.2 for some $\beta_{i} \in \mathbb{R}$ the expansions

$$
\begin{aligned}
|\vec{\Phi}|^{2} & =\frac{1}{|z|^{2}}+O\left(\log ^{2}|z|\right) \\
g_{\vec{\Phi}} & =\frac{1}{|z|^{4}}\left(1+O\left(|z|^{2}\right)\right) \\
h_{\vec{\Phi}}^{0} & =-\beta_{i} \frac{d z^{2}}{z^{2}}+O\left(\frac{1}{|z|}\right) \\
\left\langle\vec{n}_{\vec{\Phi}}, \vec{\Phi}\right\rangle & =-\beta_{i}(\log |z|+1)+O(|z|)
\end{aligned}
$$

Therefore, by Lemma 10.8, we deduce that

$$
\begin{aligned}
& g_{\vec{\Psi}}=\frac{g_{\vec{\Phi}}}{|\vec{\Phi}|^{4}}=1+O\left(|z|^{2} \log ^{2}|z|\right) \\
& H_{\vec{\Psi}}=-2\left\langle\vec{n}_{\vec{\Phi}}, \vec{\Phi}\right\rangle=2 \beta_{i}(\log |z|+1)+O(|z|) \\
& h_{\vec{\Psi}}^{0}=-\frac{1}{|\vec{\Phi}|^{2}} \vec{h}_{\vec{\Phi}}^{0}=\beta_{i} \frac{\bar{z}}{z} d z^{2}+O(|z|) .
\end{aligned}
$$

Therefore, the last equations becomes

$$
\partial \alpha=-2 \beta_{i} v\left(p_{i}\right)(\log |z|+1)+c_{2}+O(|z|)
$$




$$
\bar{\partial} \alpha=-\beta_{i} v\left(p_{i}\right) \frac{z}{\bar{z}}+c_{3}+O(|z|)
$$

Integrating the second equation, we find by Proposition C.2 of [1] (see also the appendix of [29])

$$
\alpha=-2 \beta_{i} v\left(p_{i}\right) z \log |z|+c_{3} \bar{z}+f_{0}(z)+O\left(|z|^{2}\right)
$$

where $f_{3}$ is a holomorphic function. Likewise, integrating the first equation, we get

$$
\alpha=-2 \beta_{i} v\left(p_{i}\right) z \log |z|+\left(-\beta_{i} v\left(p_{i}\right)+c_{2}\right) z+\overline{f_{1}(z)}+O\left(|z|^{2}\right),
$$

where $f_{1}$ is holomorphic. Comparing the two expansions, we deduce that

$$
\begin{aligned}
\alpha & =-2 \beta_{i} v\left(p_{i}\right) z \log |z|+\left(-\beta_{i} v\left(p_{i}\right)+c_{2}\right) z+c_{3} \bar{z}+O\left(|z|^{2}\right) \\
& =-2 \beta_{i} v\left(p_{i}\right) z \log |z|+\gamma_{1} z+\gamma_{2} \bar{z}+O\left(|z|^{2}\right)
\end{aligned}
$$

for some $\gamma_{1}, \gamma_{2} \in \mathbb{C}$.

Let us also see how to get the first order expansion at a branch point of order $m \geq 2$. We notice that in the case of inversions of minimal surfaces, the second residue can be read directly on the Weierstrass parametrisation. Indeed, we have $h_{\vec{\Phi}}^{0}=-2 \partial g \otimes \omega$ if $(g, \omega)$ is the Weierstrass data, and we deduce that there exists an integer $d+1 \leq m+1$ and $c_{1} \in \mathbb{C} \backslash\{0\}$ such that

$$
h_{\vec{\Phi}}^{0}=\frac{c_{1}}{z^{d+1}}(1+O(|z|)) d z^{2} .
$$

Up to scaling, we also have

$$
\begin{aligned}
|\vec{\Phi}|^{2} & =\frac{1}{|z|^{2 m}}(1+O(|z|)) \\
e^{2 \lambda} & =\partial_{z \bar{z}}^{2}|\vec{\Phi}|^{2}=\frac{m^{2}}{|z|^{2 m+2}}(1+O(|z|)) \\
e^{2 \mu} & =\frac{e^{2 \lambda}}{|\vec{\Phi}|^{4}}=m^{2}|z|^{2 m-2}(1+O(|z|)) \\
\vec{h}_{\vec{\Psi}}^{0} & =-\frac{1}{|\vec{\Phi}|^{2}} h_{\vec{\Phi}}^{0}=-c_{1} z^{m-d-1} \bar{z}^{m}(1+O(|z|)) d z^{2} .
\end{aligned}
$$

Therefore, the Codazzi identity implies that

$$
\partial H_{\vec{\Psi}}=g_{\vec{\Psi}}^{-1} \otimes \bar{\partial} \vec{h}_{\vec{\Psi}}^{0}=\frac{1}{m^{2}|z|^{2 m-2}}\left(-m c_{1} z^{m-d-1} \bar{z}^{m-1}\right)(1+O(|z|)) d z=-\frac{c_{1}}{m} z^{-d} d z .
$$

For $d=1$, since $H_{\vec{\Psi}}$ is real, we get $c_{1} \in \mathbb{R}$ and

$$
H_{\vec{\Psi}}=-\frac{2 c_{1}}{m} \log |z|+O(1)
$$

and if $d \neq 1$

$$
H_{\vec{\Psi}}=\frac{2}{m(d-1)} \operatorname{Re}\left(\frac{c_{1}}{z^{d-1}}\right)+O\left(|z|^{3-d}\right)=\operatorname{Re}\left(\frac{c_{0}}{z^{d-1}}\right)+O\left(|z|^{2-d}\right) .
$$

In particular, the second residue is equal to $d-1$. Now, the last equation of the system (4.4) becomes 


$$
\begin{aligned}
\bar{\partial} \alpha & =-\frac{1}{m^{2}|z|^{2 m-2}}(1+O(|z|))\left(-\bar{c}_{1} z^{m-d-1} z^{m}\right)(1+O(|z|))\left(v\left(p_{i}\right)+O(|z|)\right) \frac{d \bar{z}}{d z} \\
& =\frac{\overline{c_{1}}}{m^{2}} \frac{z}{\bar{z}^{d}}\left(v\left(p_{i}\right)+O(|z|)\right) \frac{d \bar{z}}{d z} .
\end{aligned}
$$

Assuming that $d \neq 1$ (if $d=1$, the computations are exactly the same as in the case of minimal surfaces with embedded planar ends), we deduce that

$$
\alpha=\left(-\frac{\overline{c_{1}}}{m^{2}(d-1)} \frac{z}{\bar{z}^{d-1}} v\left(p_{i}\right)+f_{1}(z)+O\left(|z|^{3-d}\right)\right) \frac{1}{d z},
$$

where $f_{1}$ is a meromorphic function. Then, we have

$$
\begin{aligned}
\partial\left(|z|^{2 m-2} \alpha\right) & =-|z|^{2 m-2} \operatorname{Re}\left(\frac{c_{0}}{z^{d-1}}\right)\left(v\left(p_{i}\right)+O(|z|)\right) \frac{d \bar{z}}{d z} \\
& =-\frac{1}{2}\left(c_{0} z^{m-d} \bar{z}^{m-1}+\overline{c_{0}} z^{m-1} \bar{z}^{m-d}\right)\left(v\left(p_{i}\right)+O(|z|)\right) \frac{d \bar{z}}{d z} .
\end{aligned}
$$

Integrating, we find that there exists a holomorphic function $f_{2}$ such that

$$
|z|^{2 m-2} \alpha=-\frac{1}{2}\left(\frac{c_{0}}{m+1-d} z^{m+1-d} \bar{z}^{m-1}+\frac{\overline{c_{0}}}{m} z^{m} \bar{z}^{m-d}\right)\left(v\left(p_{i}\right)+O(|z|)\right) \frac{1}{d z}+\overline{f_{2}(z)} \frac{1}{d z}
$$

which implies that

$$
\alpha=\left(-\frac{1}{2} \frac{c_{0}}{m+1-d} \frac{1}{z^{d-2}} v\left(p_{i}\right)-\frac{1}{2} \frac{\overline{c_{0}}}{m} \frac{z}{z^{d-1}} v\left(p_{i}\right)+\frac{\overline{f_{2}(z)}}{|z|^{2 m-2}}+O\left(|z|^{3-d}\right)\right) \frac{1}{d z} .
$$

Since

$$
c_{0}=\frac{2 c_{1}}{m(d-1)},
$$

the first expansion (4.5) becomes

$$
\alpha=\left(-\frac{\overline{c_{0}}}{m} \frac{z}{\bar{z}^{d-1}}+f_{1}(z)+O\left(|z|^{3-d}\right)\right) \frac{1}{d z}
$$

which shows by comparing with the second expansion (4.6) that $|z|^{2-2 m} \overline{f_{2}(z)}$ is holomorphic (provided that $f_{2}$ is restricted to its Taylor expansion of order $(2 m-2)+(2-d)$. Therefore, we have

$$
0=\partial_{\bar{z}}\left(\frac{\overline{f_{2}(z)}}{|z|^{2 m-2}}\right)=\frac{1}{|z|^{2 m-2}}\left(\overline{f_{2}^{\prime}(z)}-\frac{(m-1)}{\bar{z}} \overline{f_{2}(z)}\right)
$$

Since $m \geq 2$, we deduce that there exists $c_{2} \in \mathbb{C}$ such that $f_{2}(z)=\overline{c_{2}} z^{m-1}$. Finally, we get

$$
\alpha=\left(-\frac{1}{2} \frac{c_{0}}{m+1-d} \frac{1}{z^{d-2}} v\left(p_{i}\right)-\frac{1}{2} \frac{\overline{c_{0}}}{m} \frac{z}{\bar{z}^{d-1}} v\left(p_{i}\right)+\frac{c_{2}}{z^{m-1}}+O\left(|z|^{3-d}\right)\right) \frac{1}{d z} .
$$

If $c_{2} \neq 0$, we have

$$
\alpha=\left(\frac{c_{2}}{z^{m-1}}+O\left(|z|^{2-m}\right)\right) \frac{1}{d z}
$$


and the first equation is

$$
\begin{aligned}
\partial v= & \frac{1}{2}\left(-\frac{m(d-1)}{2} c_{0} z^{m-d-1} \bar{z}^{m}\right) \frac{c_{2}}{z^{m-1}} \\
& +\frac{1}{2} m^{2}|z|^{2 m-2} \operatorname{Re}\left(\frac{c_{0}}{z^{d-1}}\right) \frac{\overline{c_{2}}}{\bar{z}^{m-1}}+O\left(|z|^{m-d+1}\right) d z \\
= & -\frac{m(d-1)}{2} c_{0} z^{-d} \bar{z}^{m}+\frac{m^{2}}{4} \overline{c_{0} c_{2}} z^{m-1} \bar{z}^{1-d}+\frac{m^{2}}{4} c_{0} \overline{c_{2}} z^{m-d}+O\left(|z|^{m-d+1}\right) .
\end{aligned}
$$

This implies since there exists a holomorphic function $f_{3}$ such that

$$
\begin{aligned}
v= & v\left(p_{i}\right)+\frac{m}{4} c_{0} c_{2} z^{1-d} \bar{z}^{m}+\frac{m}{4} \overline{c_{0} c_{2}} z^{m} \bar{z}^{1-d} \\
& +\frac{m^{2}}{4(m-d+1)} c_{0} \overline{c_{2}} z^{m-d+1}+\overline{f_{3}(z)}+O\left(|z|^{m-d+1}\right) .
\end{aligned}
$$

Since $v$ is real, we deduce that

$$
v=v\left(p_{i}\right)+\frac{m}{2} \operatorname{Re}\left(c_{0} c_{2} z^{1-d} \bar{z}^{m}\right)+\frac{m^{2}}{2(m-d+1)} \operatorname{Re}\left(c_{0} \overline{c_{2}} z^{m-d+1}\right)+O\left(|z|^{m-d+2}\right) .
$$

In particular, if $d=m$, then $v \notin W^{2,2}(\Sigma)$ since $\operatorname{Re}\left(c_{0} c_{2} z^{1-m} \bar{z}^{m}\right) \notin W^{2,2}\left(\mathbb{D}^{2}\right)$, and since $W^{2,2}(\Sigma)$ is the minimal regularity we want to impose on $v$, this implies that $c_{2}=0$. Likewise, if $\vec{\Psi}$ is smooth, or $d=1$, then we just get for some $\gamma_{0} \in \mathbb{C}$

$$
v=v\left(p_{i}\right)+\frac{m}{2} \operatorname{Re}\left(c_{0} c_{2} \bar{z}^{m}\right)+\frac{m}{2} \operatorname{Re}\left(c_{0} \overline{c_{2}} z^{m}\right)=v\left(p_{i}\right)+\operatorname{Re}\left(\gamma_{0} z^{m}\right)+O\left(|z|^{m+1}\right)
$$

so we retrieve the previous condition. Furthermore, recalling that

$$
\mathscr{L}_{g}^{\perp} \vec{v}=-\left(\Delta_{g} v+|A|^{2} v\right)+4 \operatorname{Re}(\partial H \otimes \alpha),
$$

this is natural to impose that $\Delta_{g} v$ be not more singular than $|A|^{2} v$. Indeed, all terms of higher singularity will be compensated by $4 \operatorname{Re}(\partial H \otimes \alpha)$, but this will only increase the complexity without giving us further negative variations. Therefore, we will assume that $c_{2}=0$ in what follows, and coming back to the first equation of (4.4), we deduce that

$$
\alpha=\left(-\frac{1}{2} \frac{c_{0}}{m+1-d} \frac{1}{z^{d-2}} v\left(p_{i}\right)-\frac{1}{2} \frac{\overline{c_{0}}}{m} \frac{z}{\bar{z}^{d-1}} v\left(p_{i}\right)+O\left(|z|^{3-d}\right)\right) \frac{1}{d z}
$$

and we compute

$$
\begin{aligned}
\frac{1}{2} h_{0} \otimes \alpha= & \frac{1}{2}\left(-\frac{m(d-1)}{2} c_{0} z^{m-d-1} \bar{z}^{m}\right)\left(-\frac{1}{2} \frac{c_{0}}{m+1-d} \frac{1}{z^{d-2}} v\left(p_{i}\right)\right. \\
& \left.-\frac{1}{2} \frac{\bar{c}}{m} \frac{z}{z^{d-1}} v\left(p_{i}\right)+O\left(|z|^{3-d}\right)\right) d z \\
= & -\frac{1}{8}\left(\frac{m(d-1)}{m+1-d} c_{0}^{2} v\left(p_{i}\right) z^{m+1-2 d} \bar{z}^{m}\right. \\
& \left.+(d-1)\left|c_{0}\right|^{2} v\left(p_{i}\right) z^{m-d} \bar{z}^{m-d+1}\right) d z+O\left(|z|^{2 m-2 d+2}\right)
\end{aligned}
$$

and

$$
\frac{1}{2} H g \otimes \bar{\alpha}=-\frac{m^{2}}{8}\left(c_{0} z^{m-d} \bar{z}^{m-1}+\overline{c_{0}} z^{m-1} \bar{z}^{m-d}\right)\left(\frac{\overline{c_{0}}}{m+1-d} \frac{1}{\bar{z}^{d-2}} v\left(p_{i}\right)\right.
$$




$$
\begin{aligned}
& \left.+\frac{c_{0}}{m} \frac{\bar{z}}{z^{d-1}} v\left(p_{i}\right)+O\left(|z|^{3-d}\right)\right) d z \\
= & -\frac{1}{8}\left(\left(\frac{m^{2}}{m+1-d}+m\right)\left|c_{0}\right|^{2} z^{m-d} \bar{z}^{m+1-d}+m c_{0}^{2} z^{m+1-2 d} \bar{z}^{m}\right. \\
& \left.+\frac{m^{2} \bar{c}_{0}^{2}}{m+1-d} z^{m-1} \bar{z}^{m+2-2 d}+O\left(|z|^{2 m-2 d+2}\right)\right) d z .
\end{aligned}
$$

Therefore, integrating the first equation

$$
\partial v=\frac{1}{2} h_{0} \otimes \alpha+\frac{1}{2} H g \otimes \bar{\alpha}
$$

yields for some holomorphic function (it can not be meromorphic since $\left.v \in L^{2}\left(\Sigma, g_{0}\right)\right) f_{4}$ the identity

$$
\begin{aligned}
v= & v\left(p_{i}\right)-\frac{1}{8}\left(\frac{m(d-1)}{(m+1-d)(m+2-2 d)} c_{0}^{2} v\left(p_{i}\right) z^{m+2-2 d} \bar{z}^{m}\right. \\
& \left.+\frac{(d-1)}{m+1-d}\left|c_{0}\right|^{2} v\left(p_{i}\right)|z|^{2 m+2-2 d}\right) \\
& -\frac{1}{8}\left(\left(\frac{m^{2}}{(m+1-d)^{2}}+\frac{m}{m+1-d}\right)\left|c_{0}\right|^{2} v\left(p_{i}\right)|z|^{2 m-2 d+2}\right. \\
& +\frac{m}{m+2-2 d} c_{0}^{2} v\left(p_{i}\right) z^{m+1-2 d} \bar{z}^{m} \\
& \left.+\frac{m}{m+1-d} \bar{c}^{2} z^{m} \bar{z}^{m+2-2 d}\right)+\overline{f_{4}(z)}+O\left(|z|^{2 m-2 d+3}\right) \\
= & v\left(p_{i}\right)-\frac{1}{8}\left(\frac{2 m^{2}}{(m+1-d)(m+2-2 d)} \operatorname{Re}\left(c_{0}^{2} v\left(p_{i}\right) z^{m+2-2 d} \bar{z}^{m}\right)\right. \\
& \left.+\frac{2 m^{2}-(d-1)^{2}}{(m+1-d)^{2}}\left|c_{0}\right|^{2} v\left(p_{i}\right)|z|^{2 m+2-2 d}\right) \\
& +\frac{\left(z f_{4}(z)\right.}{f_{4}} O\left(|z|^{2 m-2 d+3}\right),
\end{aligned}
$$

where we have used

$$
\begin{aligned}
\frac{m(d-1)}{(m+1-d)(m+2-2 d)}+\frac{m}{m+2-2 d} & =\frac{m^{2}}{(m+1-d)(m+2-2 d)} \\
\frac{m^{2}}{(m+1-d)^{2}}+\frac{m}{m+1-d}+\frac{(d-1)}{m+1-d} & =\frac{2 m^{2}-(d-1)^{2}}{(m+1-d)^{2}}
\end{aligned}
$$

Since $v$ is real, we deduce that $f_{4}=0$ (this implies that $f_{4}$ is a real constant, but we have already written in this expansion the constant). This implies that the next order expansion of $\alpha$ is also a linear function of $v\left(p_{i}\right)$, and by an immediate induction, since $H$ and $h_{0}$ (of $\vec{\Psi}$ ) admit the following expansions thanks to Lemma 10.8

$$
\begin{aligned}
H & =\operatorname{Re}\left(\frac{c_{0}}{z^{d-1}}\right)+\sum_{j \geq 1} \sum_{k \geq 0} \operatorname{Re}\left(c_{j, k} \frac{\bar{z}^{k}}{z^{d-j-1}}\right)+\gamma_{0}|z|^{2 m-2} \log |z|+O(|z|) \\
h_{0} & =-c_{1} z^{m-d-1} \bar{z}^{m}+\sum_{i+j=2 m-d}^{m+d-3} c_{j, k} z^{j} \bar{z}^{k}+O\left(|z|^{m+d-2}\right),
\end{aligned}
$$


we deduce that the given expansions hold.

\section{Decomposition of the renormalised energy}

We fix a Willmore surface $\vec{\Psi}: \Sigma \rightarrow \mathbb{R}^{3}$ which is the inversion of a complete minimal surface $\vec{\Phi}: \Sigma \backslash\left\{p_{1}, \ldots, p_{n}\right\} \rightarrow \mathbb{R}^{3}$ of finite total curvature. We fix $v \in W^{2,2}(\Sigma)$ (such that $\vec{v}=v \vec{n}_{\vec{\Psi}}+2 \operatorname{Re}(\alpha \otimes \partial \vec{\Psi}) \in \operatorname{Var}(\vec{\Psi})$ for some $(-1,0)$-form $\left.\alpha\right)$, and as in the introduction, for all $\varepsilon>0$ small enough, we consider the following minimisation problem

$$
\inf _{w \in \mathscr{E}_{\varepsilon}\left(p_{i}\right)} \frac{1}{2} \int_{\Sigma \backslash \bar{B}_{\varepsilon}\left(p_{i}\right)}\left(\Delta_{g} w-2 K_{g} w\right)^{2} d \operatorname{vol}_{g}
$$

where the class of admissible functions is

$$
\mathscr{E}_{\varepsilon}\left(p_{i}\right)=\mathrm{W}^{2,2}\left(\Sigma \backslash \bar{B}_{\varepsilon}\left(p_{i}\right)\right) \cap\left\{w:\left\{\begin{array}{cl}
w=u & \text { on } \partial B_{\varepsilon}\left(p_{i}\right) \\
\partial_{\nu} w=\partial_{\nu} u & \text { on } \partial B_{\varepsilon}\left(p_{i}\right)
\end{array}\right\} .\right.
$$

Notice that for an end $p_{j}$ (for some $1 \leq j \leq n$ ) of multiplicity $m \geq 1$ of a complete minimal surface with finite total curvature $\vec{\Phi}: \Sigma \rightarrow\left\{p_{1}, \ldots, p_{n}\right\} \rightarrow \mathbb{R}^{n}$, in any complex chart $z: B(0,1) \subset \mathbb{C} \rightarrow \Sigma$ such that $z(0)=p_{j}$, there exists $\vec{A}_{0} \in \mathbb{C}^{n} \backslash\{0\}$ (depending on $z$ ) such that

$$
\vec{\Phi}(z)=\operatorname{Re}\left(\frac{\vec{A}_{0}}{z^{m}}\right)+O\left(|z|^{1-m}\right)
$$

for $m \geq 2$, while for $m=1$ there exists $\vec{\gamma}_{0} \in \mathbb{R}^{n}$ such that

$$
\vec{\Phi}(z)=\operatorname{Re}\left(\frac{\vec{A}_{0}}{z}\right)+\vec{\gamma}_{0} \log |z|+O(1) .
$$

Therefore, we have up to scaling

$$
e^{2 \lambda}=2\left|\partial_{z} \vec{\Phi}\right|^{2}=\frac{1}{|z|^{2(m+1)}}(1+O(|z|)) .
$$

In particular, we deduce that

$$
K_{g}=-\Delta_{g} \lambda=O\left(|z|^{2(m+1)}\right),
$$

and

$$
\mathscr{L}_{g}=\Delta_{g}-2 K_{g}=e^{-2 \lambda}(\Delta+2 \Delta \lambda)=|z|^{2(m+1)}(1+O(|z|))(\Delta+O(1)),
$$

so $\mathscr{L}_{g}$ is not elliptic in a neighbourhood of $p_{j}$. Therefore, we will have to consider another problem than (5.1).

Recall first by definition of $B_{\varepsilon}\left(p_{i}\right)$ that $B_{1}\left(p_{i}\right) \cap B_{1}\left(p_{j}\right)=\varnothing$ for all $1 \leq i \neq j \leq n$. Therefore, for all $0<\varepsilon<1$, and for all $0<\delta<\varepsilon$, and $1 \leq i \leq n$ consider the domain

$$
\Sigma_{\varepsilon, \delta}^{i}=\Sigma \backslash\left(\bar{B}_{\varepsilon}\left(p_{i}\right) \cup \bigcup_{j \neq i} \bar{B}_{\delta}\left(p_{i}\right)\right) .
$$

We will also write

$$
\Sigma_{\varepsilon}^{i}=\bigcup_{\delta>0} \Sigma_{\varepsilon, \delta}^{i}=\Sigma \backslash\left(\bar{B}_{\varepsilon}\left(p_{i}\right) \cup\left\{p_{1}, \ldots, p_{n}\right\}\right)
$$


Then $\mathscr{L}_{g}$ and $\mathscr{L}_{g}^{2}$ are strongly elliptic on $\Sigma_{\varepsilon, \delta}^{i}$ and have the uniqueness for the Cauchy problem i.e. if $\mathscr{L}_{g} u=0$ (resp. $\mathscr{L}_{g}^{2} u=0$ ) and $u=0$ on some open $U \subset \Sigma_{\varepsilon, \delta}^{i}$, then $u=0$ (this fact was first proved in general by Simons [42]), thanks to a classical theorem of Smale (see $[9,44])$ there exists $0<\varepsilon_{0}$ such that for all $0<\varepsilon<\varepsilon_{0}$, there exists $0<\delta(\varepsilon)<\varepsilon$ such that for all $0<\delta<\delta(\varepsilon)$, the operators $\mathscr{L}_{g}$ and $\mathscr{L}_{g}^{2}$ have no kernel on $\Sigma_{\varepsilon, \delta}^{i}$ for all $1 \leq i \leq n$. More precisely, the only solution of each of the two following problems

$$
\left\{\begin{aligned}
\mathscr{L}_{g} u=0 & \text { in } \Sigma_{\varepsilon, \delta}^{i} \\
u=0 & \text { on } \partial \Sigma_{\varepsilon, \delta}^{i},
\end{aligned}\right.
$$

and

$$
\left\{\begin{aligned}
\mathscr{L}_{g}^{2} u=0 & \text { in } \Sigma_{\varepsilon, \delta}^{i} \\
u=0 & \text { on } \partial \Sigma_{\varepsilon, \delta}^{i} \\
\partial_{\nu} u=0 & \text { on } \partial \Sigma_{\varepsilon, \delta}^{i}
\end{aligned}\right.
$$

is the trivial solution $u=0$. Therefore, thanks to the Fredholm alternative (see [7, IX.23]) for all $1 \leq i \leq n$ and all but finitely many $0<\varepsilon<\varepsilon_{0}$ there exists a unique minimiser $u_{\varepsilon, \delta}^{i}$ of (5.1) such that

$$
\left\{\begin{aligned}
\mathscr{L}_{g}^{2} u_{\varepsilon, \delta}^{i}=0 & \text { on } \Sigma_{\varepsilon, \delta}^{i} \\
u_{\varepsilon, \delta}^{i}=u & \text { on } \partial B_{\varepsilon}\left(p_{i}\right) \\
\partial_{\nu} u_{\varepsilon, \delta}^{i}=\partial_{\nu} u & \text { on } \partial B_{\varepsilon}\left(p_{i}\right) \\
u_{\varepsilon, \delta}^{i}=0 & \text { on } \partial B_{\delta}\left(p_{j}\right) \text { for all } 1 \leq j \neq i \leq n \\
\partial_{\nu} u_{\varepsilon, \delta}^{i}=0 & \text { on } \partial B_{\delta}\left(p_{j}\right) \text { for all } 1 \leq j \neq i \leq n
\end{aligned}\right.
$$

where $u=|\vec{\Phi}|^{2} v$ and $\mathscr{L}_{g}=\Delta_{g}-2 K_{g}$ is the Jacobi operator of the minimal surface $\vec{\Phi}$ : $\Sigma \backslash\left\{p_{1}, \ldots, p_{n}\right\} \rightarrow \mathbb{R}^{3}$. In particular, we fix $0<\varepsilon<\varepsilon_{0}$ and we assume $0<\delta<\delta_{0}(\varepsilon)<\varepsilon$. Furthermore, notice that $u_{\varepsilon, \delta}^{i}$ is the unique solution to the variational problem

$$
\inf _{w \in \mathscr{E}_{\varepsilon, \delta}\left(p_{i}\right)} \frac{1}{2} \int_{\Sigma_{\varepsilon, \delta}^{i}}\left(\Delta_{g} w-2 K_{g} w\right)^{2} d \operatorname{vol}_{g}
$$

where

$$
\mathscr{E}_{\varepsilon, \delta}\left(p_{i}\right)=W^{2,2}\left(\Sigma_{\varepsilon, \delta}^{i}\right) \cap\left\{w:\left\{\begin{array}{cl}
w=u & \text { on } \partial B_{\varepsilon}\left(p_{i}\right) \\
\partial_{\nu} w=\partial_{\nu} u & \text { on } \partial B_{\varepsilon}\left(p_{i}\right) \\
w=0 & \text { on } \partial B_{\varepsilon}\left(p_{j}\right) \text { for all } 1 \leq j \neq i \leq n \\
\partial_{\nu}=0 & \text { on } \partial B_{\varepsilon}\left(p_{j}\right) \text { for all } 1 \leq j \neq i \leq n .
\end{array}\right\}\right.
$$

\subsection{Estimate of the singular energy of the minimisers}

Recall the definition

$$
Q_{\vec{\Psi}}(\vec{v})=D^{2} W(\vec{\Psi})(\vec{v}, \vec{v}),
$$

for some admissible variation $\vec{v} \in \operatorname{Var}(\vec{\Psi})$. Fix some $1 \leq i \leq n$ and assume that $p_{i}$ has multiplicity $m \geq 1$. Up to scaling, we have $|\vec{\Phi}|^{2}=|z|^{-2 m}(1+O(|z|))$. Now assume that 
$\vec{v} \in \operatorname{Var}(\vec{\Psi}) \cap C^{\infty}\left(\Sigma, \mathbb{R}^{3}\right)$. By Theorem 4.1, we can make a decomposition

$$
u=|\vec{\Phi}|^{2} v=|\vec{\Phi}|^{2} v_{0} v\left(p_{i}\right)+\operatorname{Re}\left(\frac{\gamma_{0}}{z^{m}}\right)+O\left(|z|^{1-m}\right),
$$

which implies that

$$
\Delta_{g} u-2 K_{g} u=\left(\Delta_{g}\left(|\vec{\Phi}|^{2} v_{0}\right)-2 K_{g}|\vec{\Phi}|^{2} v_{0}\right) v\left(p_{i}\right)+O\left(|z|^{m+1}\right)=f_{0}(z) v\left(p_{i}\right)+f_{1}(z),
$$

and we get

$$
\begin{aligned}
\frac{1}{2} \int_{B_{1} \backslash \bar{B}_{\varepsilon}\left(p_{i}\right)}\left(\mathscr{L}_{g} u\right)^{2} d \operatorname{vol}_{g}= & \frac{1}{2} \int_{B_{1} \backslash \bar{B}_{\varepsilon}(0)}\left(f_{0}(z)^{2} v^{2}\left(p_{i}\right)+2 v\left(p_{i}\right) f_{1}(z)\right) \\
& \frac{m^{2}}{|z|^{2 m+2}}\left(1+\sum_{i \geq j \geq 0, i+j \geq 1} \operatorname{Re}\left(a_{i, j} z^{i} \bar{z}^{j}\right)\right) \\
= & \frac{8 \pi m^{2}}{\varepsilon^{2 m}} v^{2}\left(p_{i}\right)+4 \pi \sum_{j=1}^{m} \sum_{k \geq 0} \frac{\alpha_{j, k}}{\varepsilon^{2 m-j}} \log ^{k}\left(\frac{1}{\varepsilon}\right) v^{2}\left(p_{i}\right) \\
& +4 \pi \sum_{j=m+1}^{2 m-1} \sum_{k \geq 0} \frac{\beta_{j, k}(v)}{\varepsilon^{2 m-j}} v\left(p_{i}\right) \log ^{k}\left(\frac{1}{\varepsilon}\right)+O(1) \\
= & Q_{\varepsilon}^{i}(v)+O(1)
\end{aligned}
$$

where the $\alpha_{j, k}$ and $\beta_{j, k}(v)$ are almost all zero (meaning that they are all zero but finitely many of them).

In particular, for all smooth admissible variation $\vec{v}=-v \vec{n}_{\vec{\Psi}}+2 \operatorname{Re}(\alpha \otimes \partial \vec{\Psi})$ of $\vec{\Psi}$ as above, we deduce since the limit

$$
Q_{\vec{\Psi}}(\vec{v})=\lim _{\varepsilon \rightarrow 0}\left(\frac{1}{2} \int_{\Sigma_{\varepsilon}}\left(\mathscr{L}_{g} u\right)^{2} d \operatorname{vol}_{g}+\sum_{i=1}^{n} \int_{\partial B_{\varepsilon}\left(p_{i}\right)} \omega(u, \alpha)\right)
$$

exists and is finite that

$$
\begin{aligned}
\int_{\partial B_{\varepsilon}\left(p_{i}\right)} \omega(u, \alpha)= & -\frac{8 \pi m^{2}}{\varepsilon^{2 m}} v^{2}\left(p_{i}\right)-4 \pi \sum_{j=1}^{m} \sum_{k \geq 0} \frac{\alpha_{j, k}}{\varepsilon^{2 m-j}} \log ^{k}\left(\frac{1}{\varepsilon}\right) v^{2}\left(p_{i}\right) \\
& -4 \pi \sum_{j=m+1}^{2 m-1} \sum_{k \geq 0} \frac{\beta_{j, k}(v)}{\varepsilon^{2 m-j}} v\left(p_{i}\right) \log ^{k}\left(\frac{1}{\varepsilon}\right) \\
& +\gamma_{0}(v) v\left(p_{i}\right)+O\left(\varepsilon \log ^{N} \varepsilon\right)
\end{aligned}
$$

for some $N \geq 0$. Now, assume that $v\left(p_{i}\right)=0$. Then we can assume that $\vec{v}$ admits the expansion

$$
\vec{v}=-v \vec{n}_{\vec{\Psi}}+2 \operatorname{Re}(\alpha \otimes \partial \vec{\Psi})=\operatorname{Re}\left(\vec{\gamma}_{0} z^{\theta_{0}}\right)+O\left(|z|^{\theta_{0}+1}\right)
$$

which implies that

$$
\alpha=O(|z|) .
$$


This can also be seen directly thanks to the previous algorithm. Furthermore, we have $h_{0}=$ $O\left(|z|^{-(m+1)}\right)$, which implies that

$$
\begin{aligned}
& \Delta_{g} u+2 K_{g} u+4 \operatorname{Re}\left(g^{-1} \otimes h_{0} \otimes \bar{\partial} \alpha\right)=O\left(|z|^{m+1}\right) \\
& 2 \partial u+h_{0} \otimes \alpha=O\left(|z|^{-(m+1)}\right) .
\end{aligned}
$$

Therefore, we have

$$
\left(\Delta_{g} u+2 K_{g} u+4 \operatorname{Re}\left(g^{-1} \otimes h_{0} \otimes \bar{\partial} \alpha\right)\right)\left(2 \partial u+h_{0} \otimes \alpha\right)=O(1)
$$

Since $K_{g}=O\left(|z|^{2 m+2}\right)$, and $g=O\left(|z|^{-(2 m+2)}\right)$, we get

$$
\begin{aligned}
& g^{-1} \otimes \bar{\partial}(g \otimes \bar{\alpha})=O(1) \\
& 2 g^{-1} \otimes h_{0} \otimes \bar{\partial} u=2 m^{-2}|z|^{2 m+2} \times O\left(|z|^{-(m+1)}\right) \times O\left(|z|^{-(m+1)}\right)=O(1) \\
& K_{g} g \otimes \bar{\alpha}=O(|z|) .
\end{aligned}
$$

Therefore, we deduce that

$$
2 g^{-1} \otimes \bar{\partial}(g \otimes \bar{\alpha})\left(2 g^{-1} \otimes h_{0} \otimes \bar{\partial} u-K_{g} g \otimes \bar{\alpha}\right)=O(1) .
$$

Since $\left\langle\partial_{z} \vec{\Phi}, \vec{n}_{\vec{\Phi}}\right\rangle=0$, we deduce by the expansions

$$
\begin{aligned}
\partial_{z} \vec{\Phi} & =-m \frac{\vec{A}_{0}}{z^{m+1}}+O\left(|z|^{-m}\right) \\
\vec{n}_{\vec{\Phi}} & =\vec{n}_{\vec{\Phi}}\left(p_{i}\right)+O(|z|)=\vec{n}_{0}+O(|z|)
\end{aligned}
$$

that $\left\langle\vec{A}_{0}, \vec{n}_{0}\right\rangle=\left\langle\overrightarrow{\vec{A}}_{0}, \vec{n}_{0}\right\rangle=0$. Integrating the previous expansion, we deduce that

$$
\vec{\Phi}(z)=\operatorname{Re}\left(\frac{\vec{A}_{0}}{z^{m}}\right)+O\left(|z|^{1-m}\right),
$$

which implies that

$$
\left\langle\vec{\Phi}, \vec{n}_{\vec{\Phi}}\right\rangle=O\left(|z|^{1-m}\right) .
$$

Since $v=O\left(|z|^{m}\right)$, we deduce that $v^{2}=O\left(|z|^{2 m}\right)$ and

$$
|\vec{\Phi}|^{2} v^{2}+\frac{1}{|\vec{\Phi}|^{2}} g \otimes|\alpha|^{2}=O(1)
$$

so that

$$
\begin{array}{r}
4\left\langle\vec{\Phi}, \vec{n}_{\vec{\Phi}}\right\rangle g^{-1} \otimes h_{0} \otimes \bar{\partial}\left(|\vec{\Phi}|^{2} v^{2}+\frac{1}{|\vec{\Phi}|^{2}} g \otimes|\alpha|^{2}\right) \\
=O\left(|z|^{1-m}\right) \otimes O\left(|z|^{m+1}\right) \times O\left(|z|^{-1}\right)=O(|z|),
\end{array}
$$

and likewise,

$$
-8 g^{-1} \otimes h_{0} \otimes \bar{\partial}\left(|\vec{\Phi}|^{2}\left\langle\vec{\Phi}, \vec{n}_{\vec{\Phi}}\right\rangle v^{2}+2 \operatorname{Re}(\alpha \otimes \log |\vec{\Phi}|)|\vec{\Phi}|^{2} v\right)=O(|z|) .
$$

Finally, we have

$$
\begin{aligned}
& 4 K_{g}\left(\left\langle\vec{\Phi}, \vec{n}_{\vec{\Phi}}\right\rangle v+2 \operatorname{Re}(\alpha \otimes \partial \log |\vec{\Phi}|)\right) g \otimes \bar{\alpha} \\
& \quad=O\left(|z|^{2 m+2}\right) \times\left(O\left(|z|^{1-m}\right) \times O\left(|z|^{m}\right)+O(1)\right)|z|^{-(2 m+2)}(1+O(|z|)) \times O(|z|)=O(|z|)
\end{aligned}
$$


and we deduce that

$$
\int_{\partial B_{\varepsilon}\left(p_{i}\right)} \omega(u, \alpha)=O(\varepsilon)
$$

and

$$
Q_{\vec{\Psi}}(\vec{v})=\frac{1}{2} \int_{\Sigma}\left(\mathscr{L}_{g}\left(|\vec{\Phi}|^{2} v\right)\right)^{2} d \operatorname{vol}_{g}<\infty
$$

Therefore, we deduce the first extension of [28] to the case of branched surfaces.

Theorem 5.1 Let $\Sigma$ be a closed Riemann surface and $\vec{\Psi}: \Sigma \rightarrow \mathbb{R}^{3}$ be a branched Willmore surface. Assume that $\vec{\Psi}$ is the inversion of a complete minimal surface with finite total curvature $\vec{\Phi}: \Sigma \backslash\left\{p_{1}, \ldots, p_{n}\right\} \rightarrow \mathbb{R}^{3}$. Then we have

$$
\operatorname{Ind}_{W}(\vec{\Psi}) \leq n=\frac{1}{4 \pi} W(\vec{\Psi})-\frac{1}{2 \pi} \int_{\Sigma} K_{g_{\vec{\Psi}}} d \operatorname{vol}_{g_{\vec{\Psi}}}+\chi(\Sigma) .
$$

Proof Write $g=\vec{\Phi}^{*} g_{\mathbb{R}^{3}}$ be the induced metric on $\Sigma \backslash\left\{p_{1}, \ldots, p_{n}\right\}$. The preceding argument shows that for $\vec{v} \in \operatorname{Var}(\vec{\Psi}) \cap C^{\infty}(\Sigma)$, if $v=\left\langle\vec{v}, \vec{n}_{\vec{\Psi}}\right\rangle$, and $v\left(p_{i}\right)=0$ for all $1 \leq i \leq n$, we have

$$
Q_{\vec{\Psi}}(\vec{v})=\frac{1}{2} \int_{\Sigma}\left(\mathscr{L}_{g}\left(|\vec{\Phi}|^{2} v\right)\right)^{2} d \operatorname{vol}_{g} \geq 0 .
$$

Now, let $\vec{v} \in \operatorname{Var}(\vec{\Psi})$ be an arbitrary variation such that $v\left(p_{i}\right)=0$ for all $1 \leq i \leq n$ (where $\left.v=\left\langle\vec{v}, \vec{n}_{\vec{\Psi}}\right\rangle\right)$. Now, let $\left\{\vec{v}_{k}\right\}_{k \in \mathbb{N}} \in C^{\infty}\left(\Sigma, \mathbb{R}^{3}\right)$ such that

$$
\vec{v}_{k} \underset{k \rightarrow \infty}{\longrightarrow} \vec{v} \quad \text { in } W^{2,2}\left(\Sigma, \mathbb{R}^{3}\right) .
$$

In particular, we have by the Sobolev embedding $W^{2,2}\left(\Sigma, \mathbb{R}^{3}\right) \hookrightarrow C^{0}\left(\Sigma, \mathbb{R}^{3}\right)$, the convergence $\vec{v}_{k} \underset{k \rightarrow \infty}{\longrightarrow} \vec{v}$ in $C^{0}(\Sigma)$. Furthermore, if

$$
\vec{u}_{k}=|\vec{\Phi}|^{2} \vec{v}_{k}-2\left\langle\vec{\Phi}, \vec{v}_{k}\right\rangle \vec{\Phi}
$$

and $u_{k}=-\left\langle\vec{u}_{k}, \vec{n}_{\vec{\Phi}}\right\rangle=|\vec{\Phi}|^{2} v_{k}$, we have we have $\mathscr{L}_{g} u_{k}=-\mathscr{L}_{g}^{\perp} \vec{u}_{k} \underset{k \rightarrow \infty}{\longrightarrow}-\mathscr{L}_{g}^{\perp} \vec{u}=$ $\mathscr{L}_{g} u$ in $L_{\text {loc }}^{2}\left(\Sigma \backslash\left\{p_{1}, \ldots, p_{n}\right\}\right)$. Then up to a subsequence, we deduce that (up to taking a subsequence) $\nabla^{2} v_{k} \underset{k \rightarrow \infty}{\longrightarrow} \nabla^{2} v$ almost everywhere on $\Sigma$. In $U_{i}$ we have an expansion for some $\vec{\gamma}_{i}^{k}, \vec{\gamma}_{j_{1}, j_{2}}^{k} \in \mathbb{C}^{3}$ (as $\vec{\Phi}$ is smooth)

$$
\vec{v}_{k}=\vec{v}_{k}\left(p_{i}\right)+\sum_{\substack{j_{1}, j_{2} \geq 0 \\ 1 \leq j_{1}+j_{2} \leq m_{i}}} \operatorname{Re}\left(\vec{\gamma}_{j_{1}, j_{2}}^{k} z^{j_{1}} \bar{z}^{j_{2}}\right)+\operatorname{Re}\left(\vec{\gamma}_{i}^{k} z^{m_{i}}\right)+O\left(|z|^{m_{i}+1}\right) .
$$

As $\vec{v}_{k} \underset{k \rightarrow \infty}{\longrightarrow} \vec{v}$ and since $\vec{v}$ is admissible, we deduce (as $\vec{v}\left(p_{i}\right)=0$ ) that $\vec{v}_{k}\left(p_{i}\right) \underset{k \rightarrow \infty}{\longrightarrow} 0$ and $\vec{\gamma}_{i, j_{1}, j_{2}}^{k} \underset{k \rightarrow \infty}{\longrightarrow} 0$ for all $1 \leq j_{2}+j_{2} \leq m_{i}$. Finally, this implies that if $\rho_{i}$ is a cutoff function such that $\rho_{i}=1$ on $\varphi_{i}^{-1}(B(0,1 / 2)) \subset U_{i}$ and $\operatorname{supp}\left(\rho_{i}\right) \subset U_{i}$, and

$$
\widetilde{\vec{v}}_{k}=\vec{v}_{k}-\sum_{i=1}^{n} \rho_{i}\left\{\vec{v}_{k}\left(p_{i}\right)+\sum_{\substack{j_{1}, j_{2} \geq 0 \\ 1 \leq j_{1}+j_{2} \leq m_{i}}} \operatorname{Re}\left(\vec{\gamma}_{i, j_{1}, j_{2}}^{k} \varphi_{i}^{j_{1}} \bar{\varphi}_{i} j_{2}\right)\right\} \in C^{\infty}(\Sigma),
$$


also satisfies

$$
\widetilde{\vec{v}}_{k}\left(p_{i}\right)=0 \quad \text { for all } \quad 1 \leq i \leq n, \quad \text { and } \quad \widetilde{\vec{v}}_{k} \underset{k \rightarrow \infty}{\longrightarrow} \vec{v} \quad \text { strongly in } W^{2,2}(\Sigma) .
$$

We deduce that $\widetilde{\vec{v}}_{k}$ is an admissible variation of $\vec{\Psi}$, and by the preceding discussion we have if $\widetilde{v}_{k}=\left\langle\widetilde{\vec{v}}_{k}, \vec{n}_{\vec{\Psi}}\right\rangle$, and $\widetilde{u}_{k}=|\vec{\Phi}|^{2} \widetilde{v}_{k}$,

$$
Q_{\vec{\Psi}}\left(\widetilde{v}_{k}\right)=\frac{1}{2} \int_{\Sigma}\left(\mathscr{L}_{g} \widetilde{u}_{k}\right)^{2} d \operatorname{vol}_{g} \geq 0 .
$$

Now, by the strong $W^{2,2}$ convergence and as $\widetilde{\vec{v}}_{k}$ is admissible, we have (see for example the explicit formula for $Q_{\vec{\Psi}}$ in [28])

$$
Q_{\vec{\Psi}}\left(\widetilde{\vec{v}}_{k}\right) \underset{k \rightarrow \infty}{\longrightarrow} Q_{\vec{\Psi}}(\vec{v})
$$

Then (5.9) implies that $Q_{\vec{\Psi}}(\vec{v}) \geq 0$, but notice also that by Fatou lemma

$$
Q_{\vec{\Psi}}(\vec{v})=\liminf _{k \rightarrow \infty} Q_{\vec{\Psi}}\left(\widetilde{\vec{v}}_{k}\right) \geq \frac{1}{2} \int_{\Sigma} \liminf _{k \rightarrow \infty}\left(\mathscr{L}_{g} \widetilde{u}_{k}\right)^{2} d \operatorname{vol}_{g}=\frac{1}{2} \int_{\Sigma}\left(\mathscr{L}_{g} u\right)^{2} d \operatorname{vol}_{g} \geq 0
$$

This observation concludes the proof of the theorem, as the last equality in (5.7) comes from the Li-Yau inequality [21] and the Jorge-Meeks formula [18].

The following theorem is the analogous of Theorem V.1, 2, 3 [4]. Here, the vortices are already fixed and correspond to the points $p_{1}, \ldots, p_{n} \in \Sigma$ where the metric of the corresponding minimal surface degenerates. We first obtain an estimate of the singular energy by a geometric argument, and show that the Jacobi operator of the minimiser $u_{\varepsilon, \delta}^{i}$ is bounded in $L^{2}$ away from $p_{i}$. This will allow us to pass to the limit to a limit function as $\delta \rightarrow 0$ and $\varepsilon \rightarrow 0$.

Theorem 5.2 Let $0<\varepsilon<\varepsilon_{0}$ and $0<\delta<\delta(\varepsilon)<\varepsilon$ and $u_{\varepsilon, \delta}^{i}$ be the unique solution of (5.4). Then there exists a non-decreasing function $\omega: \mathbb{R}_{+} \rightarrow \mathbb{R}_{+}$which is continuous at 0 and such that $\omega(0)=0$ (independent of $\varepsilon$ and $\delta$ ) such that

$$
\left|\frac{1}{2} \int_{\Sigma_{\varepsilon, \delta}^{i}}\left(\mathscr{L}_{g} u_{\varepsilon, \delta}^{i}\right)^{2} d \operatorname{vol}_{g}-Q_{\varepsilon}^{i}(v)\right| \leq \omega\left(\|v\|_{\mathrm{W}^{2,2}(\Sigma)}\right)
$$

and

$$
\frac{1}{2} \int_{\Sigma_{\varepsilon_{0}, \delta}}\left(\mathscr{L}_{g} u_{\varepsilon, \delta}^{i}\right)^{2} d \operatorname{vol}_{g} \leq \omega\left(\|v\|_{\mathrm{W}^{2,2}(\Sigma)}\right) .
$$

Proof Recalling that $\Sigma_{\varepsilon}=\Sigma \backslash \bigcup_{i=1}^{n} \bar{B}_{\varepsilon}\left(p_{i}\right)$, we define the continuous bilinear form $B_{\varepsilon}$ : $W^{2,2}\left(\Sigma_{\varepsilon}\right) \times W^{2,2}\left(\Sigma_{\varepsilon}\right) \rightarrow \mathbb{R}$ by

$$
B_{\varepsilon}\left(u_{1}, u_{2}\right)=\frac{1}{2} \int_{\Sigma_{\varepsilon}} \mathscr{L}_{g} u_{1} \mathscr{L}_{g} u_{2} d \operatorname{vol}_{g}
$$

and let $Q_{\varepsilon}: W^{2,2}\left(\Sigma_{\varepsilon}\right) \rightarrow \mathbb{R}$ be the associated quadratic form. Then we have

$$
Q_{\vec{\Psi}}(\vec{v})=\lim _{\varepsilon \rightarrow 0}\left(Q_{\varepsilon}(u)-\sum_{i=1}^{n} Q_{\varepsilon}^{i}(v)\right)
$$


and the limit is well-defined. Now, fix a cutoff function $\rho_{i} \geq 0$ such that

$$
\rho_{i}=1 \quad \text { on } B_{\varepsilon_{0} / 2}\left(p_{i}\right), \quad \text { and } \operatorname{supp}\left(\rho_{i}\right) \subset B_{\varepsilon_{0}}\left(p_{i}\right) .
$$

Notice in particular that for all $1 \leq i \leq n, 0<\varepsilon<\varepsilon_{0}$ and $0<\delta<\delta(\varepsilon)$, we have

$$
\begin{aligned}
\frac{1}{2} \int_{\Sigma_{\varepsilon, \delta}^{i}}\left(\mathscr{L}_{g} u_{\varepsilon, \delta}^{i}\right)^{2} d \operatorname{vol}_{g} & \leq \frac{1}{2} \int_{\Sigma_{\varepsilon, \delta}}\left(\mathscr{L}_{g}\left(\rho_{i} u\right)\right)^{2} d \operatorname{vol}_{g} \\
& =\frac{1}{2} \int_{B_{\varepsilon_{0}} \backslash \bar{B}_{\varepsilon}\left(p_{i}\right)}\left(\mathscr{L}_{g}\left(\rho_{i} u\right)\right)^{2} d \operatorname{vol}_{g}=Q_{\varepsilon}^{i}(v)+O(1) .
\end{aligned}
$$

Now, if $0<\varepsilon<\varepsilon_{0}$ and $0<\delta<\delta(\varepsilon)<\varepsilon$ define

$$
u_{\varepsilon, \delta}=u-\sum_{i=1}^{n} u_{\varepsilon, \delta}^{i}
$$

We have

$$
\begin{aligned}
Q_{\varepsilon}(u) & =B_{\varepsilon}\left(u_{\varepsilon, \delta}+\sum_{i=1}^{n} u_{\varepsilon, \delta}^{i}, u_{\varepsilon, \delta}+\sum_{i=1}^{n} u_{\varepsilon, \delta}^{i}\right) \\
& =Q_{\varepsilon}\left(u_{\varepsilon, \delta}, u_{\varepsilon, \delta}\right)+\sum_{i=1}^{n} Q_{\varepsilon}\left(u_{\varepsilon, \delta}^{i}\right)+2 \sum_{i=1}^{n} B_{\varepsilon}\left(u_{\varepsilon, \delta}, u_{\varepsilon, \delta}^{i}\right)+\sum_{1 \leq i \neq j \leq n} B_{\varepsilon}\left(u_{\varepsilon, \delta}^{i}, u_{\varepsilon, \delta}^{j}\right) .
\end{aligned}
$$

Integrating by parts, we find

$$
B_{\varepsilon}\left(u_{\varepsilon, \delta}, u_{\varepsilon, \delta}^{i}\right)=\frac{1}{2} \int_{\partial B_{\varepsilon}\left(p_{i}\right)}\left(u_{\varepsilon, \delta} \partial_{\nu}\left(\mathscr{L}_{g} u_{\varepsilon, \delta}^{i}\right)-\partial_{\nu} u_{\varepsilon, \delta}\left(\mathscr{L}_{g} u_{\varepsilon, \delta}^{i}\right)\right) d \mathscr{H}^{1} .
$$

Since

$$
u_{\varepsilon, \delta}=-\sum_{j \neq i} u_{\varepsilon, \delta}^{j}, \quad \partial_{\nu} u_{\varepsilon, \delta}=-\sum_{j \neq i} \partial_{\nu} u_{\varepsilon, \delta}^{j} \quad \text { on } \partial B_{\varepsilon}\left(p_{i}\right)
$$

we deduce that $B_{\varepsilon}\left(u_{\varepsilon, \delta}, u_{\varepsilon, \delta}^{i}\right)$ does not contain a quadratic term of the form $C_{\varepsilon} v^{2}\left(p_{i}\right)$, since the functions $u_{\varepsilon, \delta}^{j}(j \neq i)$ are independent of $v\left(p_{i}\right)$. A similar argument applies for $B_{\varepsilon}\left(u_{\varepsilon}^{i}, u_{\varepsilon}^{j}\right)$ $(i \neq j)$, so we deduce by (3.2) that the only possibility for the limit (5.12) to be finite is that

$$
Q_{\varepsilon}\left(u_{\varepsilon}^{i}\right)=Q_{\varepsilon}^{i} v^{2}\left(p_{i}\right)+O(1)
$$

where $O(1)$ is a quantity bounded independently of $0<\varepsilon<\varepsilon_{0}$ and $0<\delta<\delta(\varepsilon)$. Therefore, combining (5.14) with (5.13), we deduce that for all $0<\delta<\delta(\varepsilon)<\varepsilon$

$$
\frac{1}{2} \int_{\Sigma_{\varepsilon, \delta}}\left(\mathscr{L}_{g} u_{\varepsilon, \delta}^{i}\right)^{2} d \operatorname{vol}_{g}=Q_{\varepsilon}^{i}(v)+O(1),
$$

where $O(1)$ is a quantity bounded independently of $0<\varepsilon<\varepsilon_{0}$ and $0<\delta<\delta(\varepsilon)$. Therefore, we deduce that

$$
\begin{aligned}
Q_{\varepsilon}^{i}(v)+O(1)= & \int_{\Sigma_{\varepsilon, \delta}^{i}}\left(\mathscr{L}_{g}\left(\rho_{i} u\right)\right)^{2} d \operatorname{vol}_{g}=\int_{\Sigma_{\varepsilon, \delta}^{i}}\left(\mathscr{L}_{g}\left(\rho_{i} u-u_{\varepsilon, \delta}^{i}\right)\right)^{2} d \operatorname{vol}_{g} \\
& +2 \int_{\Sigma_{\varepsilon, \delta}^{i}} \mathscr{L}_{g}\left(\rho_{i} u-u_{\varepsilon, \delta}^{i}\right) \mathscr{L}_{g}\left(u_{\varepsilon, \delta}^{i}\right) d \operatorname{vol}_{g}+\int_{\Sigma_{\varepsilon, \delta}^{i}}\left(\mathscr{L}_{g} u_{\varepsilon, \delta}^{i}\right)^{2} d \operatorname{vol}_{g}
\end{aligned}
$$




$$
\begin{aligned}
= & \int_{\Sigma_{\varepsilon, \delta}^{i}}\left(\mathscr{L}_{g}\left(\rho_{i} u-u_{\varepsilon, \delta}^{i}\right)\right)^{2} d \operatorname{vol}_{g} \\
& +2 \int_{\Sigma_{\varepsilon, \delta}^{i}} \mathscr{L}_{g}\left(\rho_{i} u-u_{\varepsilon, \delta}^{i}\right) \mathscr{L}_{g}\left(u_{\varepsilon, \delta}^{i}\right) d \operatorname{vol}_{g}+Q_{\varepsilon}^{i}(v)+O(1) .
\end{aligned}
$$

Furthermore, the boundary conditions imply that $u_{\varepsilon, \delta}^{i}=u=\rho_{i} u$ on $\partial B_{\varepsilon}\left(p_{i}\right)$ and $\partial_{\nu} u_{\varepsilon, \delta}^{i} u=$ $\partial_{\nu} i=\partial_{\nu}\left(\rho_{i} u\right)=0$, while for all $j \neq i, u_{\varepsilon, \delta}^{i}=\partial_{\nu} u_{\varepsilon, \delta}^{i}=\rho_{i} u=\partial_{\nu}\left(\rho_{i} u\right)=0$. Therefore, we deduce as $\mathscr{L}_{g}^{2} u_{\varepsilon, \delta}^{i}=0$ that

$$
\begin{aligned}
& \int_{\Sigma_{\varepsilon, \delta}^{i}} \mathscr{L}_{g}\left(\rho_{i} u-u_{\varepsilon, \delta}^{i}\right) \mathscr{L}_{g}\left(u_{\varepsilon, \delta}^{i}\right) d \operatorname{vol}_{g}=\int_{\Sigma_{\varepsilon, \delta}^{i}}\left(\rho_{i} u-u_{\varepsilon, \delta}^{i}\right) \mathscr{L}_{g}^{2} u_{\varepsilon, \delta}^{i} d \operatorname{vol}_{g} \\
& \quad+\int_{\partial B_{\varepsilon}\left(p_{i}\right)}\left(\rho_{i} u-u_{\varepsilon, \delta}^{i}\right) \partial_{\nu}\left(\mathscr{L}_{g} u_{\varepsilon, \delta}^{i}\right)-\partial_{\nu}\left(\rho_{i} u-u\right) \mathscr{L}_{g} u_{\varepsilon, \delta}^{i} d \mathscr{H}^{1} \\
& \quad+\sum_{j \neq i} \int_{\partial B_{\delta}\left(p_{j}\right)}\left(\rho_{i} u-u_{\varepsilon, \delta}^{i}\right) \partial_{\nu}\left(\mathscr{L}_{g} u_{\varepsilon, \delta}^{i}\right)-\partial_{\nu}\left(\rho_{i} u-u\right) \mathscr{L}_{g} u_{\varepsilon, \delta}^{i} d \mathscr{H}^{1} \\
& =0
\end{aligned}
$$

Therefore, (5.15) and (5.16) imply that

$$
\int_{\Sigma_{\varepsilon, \delta}}\left(\mathscr{L}_{g}\left(\rho_{i}-u_{\varepsilon, \delta}^{i}\right)\right)^{2} d \operatorname{vol}_{g}=O(1)
$$

and as $\operatorname{supp}\left(\rho_{i}\right) \subset B_{\varepsilon_{0}}\left(p_{i}\right)$, we deduce that

$$
\int_{\Sigma_{\varepsilon_{0}, \delta}}\left(\mathscr{L}_{g} u_{\varepsilon, \delta}^{i}\right)^{2} d \operatorname{vol}_{g}=O(1),
$$

or in other words

$$
\limsup _{\varepsilon \rightarrow 0} \limsup _{\delta \rightarrow 0} \int_{\Sigma_{\varepsilon_{0}, \delta}}\left(\mathscr{L}_{g} u_{\varepsilon, \delta}^{i}\right)^{2} d \operatorname{vol}_{g}<\infty .
$$

Furthermore, as the error terms are continuous in $v \in W^{2,2}(\Sigma)$ (such that $\vec{v}=v \vec{n}_{\vec{\Psi}}$ ), we deduce that there exists a modulus of continuity $\omega=\omega_{\vec{\Psi}}: \mathbb{R}_{+} \rightarrow \mathbb{R}_{+}$independent of $0<\varepsilon<\varepsilon_{0}$ and $0<\delta<\delta(\varepsilon)<\varepsilon$ (that we can take non-decreasing and continuous at 0 ) such that

$$
\left|\frac{1}{2} \int_{\Sigma_{\varepsilon, \delta}}\left(\mathscr{L}_{g} u_{\varepsilon, \delta}^{i}\right)^{2} d \operatorname{vol}_{g}-Q_{\varepsilon}^{i}(v)\right| \leq \omega\left(\|v\|_{\mathrm{W}^{2,2}(\Sigma)}\right)
$$

and

$$
\frac{1}{2} \int_{\Sigma_{\varepsilon_{0}}, \delta}\left(\mathscr{L}_{g} u_{\varepsilon, \delta}^{i}\right)^{2} d \operatorname{vol}_{g} \leq \omega\left(\|v\|_{\mathrm{W}^{2,2}(\Sigma)}\right) .
$$

This concludes the proof of the theorem.

Remark 5.3 Notice that the preceding proof implies that the limits of $B_{\varepsilon}\left(u_{\varepsilon, \delta}, u_{\varepsilon, \delta}^{i}\right)$ and $B_{\varepsilon}\left(u_{\varepsilon, \delta}^{i}, u_{\varepsilon, \delta}^{j}\right)(i \neq j)$ are well-defined as $\varepsilon \rightarrow 0$ (and $0<\delta<\delta(\varepsilon)<\varepsilon$ ). 


\subsection{Local estimates near the ends}

As the operators $\mathscr{L}_{g}$ and $\mathscr{L}_{g}^{2}$ are uniformly elliptic on $\Sigma_{\varepsilon}$ for all $\varepsilon>0$, the only difficult estimates come from the asymptotic behaviour near the vortices $p_{i}$ (for $1 \leq i \leq n$ ). As the estimates depend on the chart, we fix some covering $\left(U_{1}, \ldots, U_{n}\right) \subset \Sigma$ by domains of charts $\Sigma$ such that $p_{i} \in U_{i}$ for all $1 \leq i \leq n$ and all estimates will be taken with respect to a complex chart $\varphi_{i}: U_{i} \rightarrow B(0,1) \subset \mathbb{C}$ such that $\varphi_{i}\left(p_{i}\right)=0$.

Theorem 5.4 Let $1 \leq i \leq n$ be a fixed integer and $u_{\varepsilon, \delta}^{i}$ be the solution of (5.4) for some $0<\varepsilon<\varepsilon_{0}$ and $0<\delta<\delta(\varepsilon)$. Let $1 \leq j \neq i \leq n$ and assume that the end of $\vec{\Phi}$ has multiplicity $m \geq 1$, and define in the chart $U_{j}$ the function $v_{\varepsilon, \delta}^{i}=e^{-\lambda} u_{\varepsilon, \delta}^{i}$. Then there exists real analytic functions $\zeta_{0}, \zeta_{2}: B(0,1) \rightarrow \mathbb{R}$ and $\vec{\zeta}_{1}, \vec{\zeta}_{3}: B(0,1) \rightarrow \mathbb{R}^{2}$ and a universal constant $C=C\left(U_{j}, \vec{\Psi}\right)>0$ depending only on the chosen chart $U_{j}$ around $p_{j}$ and on $\vec{\Psi}$ such that

$$
\begin{aligned}
& \int_{B_{1} \backslash \bar{B}_{\delta}(0)}\left(\Delta v_{\varepsilon, \delta}^{i}-2(m+1)\left(\frac{x}{|x|^{2}}+\nabla \zeta_{0}\right) \cdot \nabla v_{\varepsilon, \delta}^{i}+\frac{(m+1)^{2}}{|x|^{2}}\left(1+x \cdot \vec{\zeta}_{1}\right) v_{\varepsilon, \delta}^{i}\right)^{2} d x \\
& \leq \int_{\Sigma_{\varepsilon_{0}, \delta}}\left(\mathscr{L}_{g} u_{\varepsilon, \delta}^{i}\right)^{2} d \mathrm{vol}_{g} \leq C \omega\left(\|v\|_{\mathrm{W}^{2,2}(\Sigma)}\right) \\
& \int_{B_{1} \backslash \bar{B}_{\delta}(0)}\left(\Delta v_{\varepsilon, \delta}^{i}+(m+1)(m-1) \frac{v_{\varepsilon, \delta}^{i}}{|x|^{2}}\right)^{2} d x \\
& \quad+4(m+1)(m-1) \int_{B_{1} \backslash \bar{B}_{\delta}(0)}\left(\frac{x}{|x|^{2}} \cdot \nabla v_{\varepsilon, \delta}^{i}-\frac{v_{\varepsilon, \delta}^{i}}{|x|^{2}}\right)^{2} d x \\
& \quad-\int_{B_{1} \backslash \bar{B}_{\delta}(0)}\left(\nabla \zeta_{2} \cdot \nabla v_{\varepsilon, \delta}^{i}-\frac{x}{|x|^{2}} \cdot \vec{\zeta}_{3} v_{\varepsilon, \delta}^{i}\right)^{2} d x \leq C \omega\left(\|v\|_{\mathrm{W}^{2,2}(\Sigma)}\right) .
\end{aligned}
$$

Proof As the end has multiplicity $m \geq 1$, there exists $\alpha_{i}>0$ and $\alpha_{0} \in \mathbb{C}$ such that

$$
\begin{aligned}
& e^{2 \lambda}=\alpha_{i}^{2}|z|^{-2(m+1)}\left(1+2 \operatorname{Re}\left(\alpha_{0} z\right)+O\left(|z|^{2}\right)\right) \\
& e^{2 \lambda} K_{g}=O(1) .
\end{aligned}
$$

Furthermore, let $\zeta: B(0,1) \rightarrow \mathbb{R}$ be the real analytic function such that

$$
\lambda(z)=-(m+1) \log |z|+\zeta(z) .
$$

Notice that $\zeta$ is real-analytic by the Weierstrass parametrisation [11]. Then we have

$$
\begin{aligned}
|\nabla \lambda|^{2} & =\frac{(m+1)^{2}}{|x|^{2}}-2(m+1) \frac{x}{|x|^{2}} \cdot \nabla \zeta+|\nabla \zeta|^{2} \\
& =\frac{(m+1)^{2}}{|x|^{2}}\left(1-\frac{2}{(m+1)} x \cdot \nabla \zeta+\frac{1}{(m+1)^{2}}|x|^{2}|\nabla \zeta|^{2}\right) .
\end{aligned}
$$

Therefore, we have if $u_{\varepsilon, \delta}^{i}=e^{\lambda} v_{\varepsilon, \delta}^{i}$ as $\Delta \zeta=-e^{2 \lambda} K_{g}$ for all $0<\kappa<1$

$$
\begin{aligned}
& \int_{B_{1} \backslash \bar{B}_{\delta}(0)}\left(\Delta_{g} u_{\varepsilon, \delta}^{i}-2 K_{g} v_{\varepsilon, \delta}^{i}\right)^{2} d \operatorname{vol}_{g}=\int_{B_{1} \backslash \bar{B}_{\delta}(0)}\left(e^{-\lambda} \Delta\left(e^{\lambda} v_{\varepsilon, \delta}^{i}\right)-2 e^{2 \lambda} K_{g} v_{\varepsilon, \delta}^{i}\right)^{2} d \operatorname{vol}_{g} \\
& =\int_{B_{1} \backslash \bar{B}_{\delta}(0)}\left(\Delta v_{\varepsilon, \delta}^{i}+2\left\langle\nabla \lambda, \nabla v_{\varepsilon, \delta}^{i}\right\rangle+\left(|\nabla \lambda|^{2}-3 e^{2 \lambda} K_{g}\right) v_{\varepsilon, \delta}^{i}\right)^{2} d x
\end{aligned}
$$




$$
\begin{aligned}
= & \int_{B_{1} \backslash \bar{B}_{\delta}(0)}\left(\Delta v_{\varepsilon, \delta}^{i}-2(m+1)\left(\frac{x}{|x|^{2}}-\frac{1}{(m+1)} \nabla \zeta\right) \cdot \nabla v_{\varepsilon, \delta}^{i}\right. \\
& \left.+\frac{(m+1)^{2}}{|x|^{2}}\left(1-\frac{2}{(m+1)} x \cdot \nabla \zeta+\frac{1}{(m+1)^{2}}|x|^{2}\left(|\nabla \zeta|^{2}+3 \Delta \zeta\right)\right) v_{\varepsilon, \delta}^{i}\right)^{2} d x \\
\geq & (1-\kappa) \int_{B_{1} \backslash \bar{B}_{\delta}(0)}\left(\Delta v_{\varepsilon, \delta}^{i}-2(m+1) \frac{x}{|x|^{2}} \cdot \nabla v_{\varepsilon, \delta}^{i}+\frac{(m+1)^{2}}{|x|^{2}} u\right)^{2} d x \\
& +\left(1-\frac{1}{\kappa}\right) \frac{1}{(m+1)^{4}} \int_{B_{1} \backslash \bar{B}_{\delta}(0)}\left(2(m+1)^{3} \nabla \zeta \cdot \nabla v_{\varepsilon, \delta}^{i}\right. \\
& \left.+\left(-2(m+1) \frac{x}{|x|^{2}} \cdot \nabla \zeta+\left(|\nabla \zeta|^{2}+3 \Delta \zeta\right)\right) v_{\varepsilon, \delta}^{i}\right)^{2} d x,
\end{aligned}
$$

where we used the inequality for all $a, b \in \mathbb{R}$ and $0<\kappa<1$

$$
(a+b)^{2} \geq(1-\kappa) a^{2}+\left(1-\frac{1}{\kappa}\right) b^{2} .
$$

In particular, the first estimate follows directly from (5.11) of Theorem 5.2, with

$$
\begin{aligned}
& \zeta_{0}=-\frac{1}{(m+1)} \zeta, \\
& \zeta_{1}=-\frac{2}{(m+1)^{2}} \nabla \zeta+\frac{x}{(m+1)^{2}}\left(|\nabla \zeta|^{2}+3 \Delta \zeta\right) .
\end{aligned}
$$

Now, thanks to the computations of Lemma, we have

$$
\begin{aligned}
& \int_{B_{1} \backslash \bar{B}_{\delta}(0)}\left(\Delta v_{\varepsilon, \delta}^{i}-2(m+1) \frac{x}{|x|^{2}} \cdot \nabla v_{\varepsilon, \delta}^{i}+\frac{(m+1)^{2}}{|x|^{2}} u\right)^{2} d x \\
& =\int_{B_{1} \backslash \bar{B}_{\delta}(0)}\left(\Delta v_{\varepsilon, \delta}^{i}+(m+1)(m-1) \frac{v_{\varepsilon, \delta}^{i}}{|x|^{2}}\right)^{2} d x \\
& +4(m+1)(m-1) \int_{B_{1} \backslash \bar{B}_{\delta}(0)}\left(\frac{x}{|x|^{2}} \cdot \nabla v_{\varepsilon, \delta}^{i}-\frac{v_{\varepsilon, \delta}^{i}}{|x|^{2}}\right)^{2} d x \\
& -\int_{S^{1}}\left(v_{\varepsilon, \delta}^{i} \partial_{\nu}\left(\mathscr{L}_{m} v_{\varepsilon, \delta}^{i}\right)-\partial_{\nu} v_{\varepsilon, \delta}^{i} \mathscr{L}_{m} v_{\varepsilon, \delta}^{i}\right) d \mathscr{H}^{1} \\
& +\int_{S^{1}}\left(v_{\varepsilon, \delta}^{i} \partial_{\nu}\left(\Delta v_{\varepsilon, \delta}^{i}\right)-\partial_{\nu}\left(v_{\varepsilon, \delta}^{i}\right) \Delta v_{\varepsilon, \delta}^{i}\right) d \mathscr{H}^{1} \\
& +4(m+1)(m-1) \int_{S^{1}}\left(\left(v_{\varepsilon, \delta}^{i}\right)^{2}-v_{\varepsilon, \delta}^{i} \partial_{\nu}^{2} v_{\varepsilon, \delta}^{i}\right) d \mathscr{H}^{1} \\
& =\int_{B_{1} \backslash \bar{B}_{\delta}(0)}\left(\Delta v_{\varepsilon, \delta}^{i}+(m+1)(m-1) \frac{v_{\varepsilon, \delta}^{i}}{|x|^{2}}\right)^{2} d x \\
& +4(m+1)(m-1) \int_{B_{1} \backslash \bar{B}_{\delta}(0)}\left(\frac{x}{|x|^{2}} \cdot \nabla v_{\varepsilon, \delta}^{i}-\frac{v_{\varepsilon, \delta}^{i}}{|x|^{2}}\right)^{2} d x \\
& -\int_{S^{1}}\left(v_{\varepsilon, \delta}^{i} \partial_{\nu}\left(\left(\mathscr{L}_{m}-\Delta\right) v_{\varepsilon, \delta}^{i}\right)-\partial_{\nu} v_{\varepsilon, \delta}^{i}\left(\mathscr{L}_{m}-\Delta\right) v_{\varepsilon, \delta}^{i}\right) d \mathscr{H}^{1} \\
& +4(m+1)(m-1) \int_{S^{1}}\left(\left(v_{\varepsilon, \delta}^{i}\right)^{2}-v_{\varepsilon, \delta}^{i} \partial_{\nu}^{2} v_{\varepsilon, \delta}^{i}\right) d \mathscr{H}^{1} .
\end{aligned}
$$


Now, if

$$
\tilde{\mathscr{L}}_{g}=e^{\lambda} \mathscr{L}_{g}\left(e^{\lambda} \cdot\right)
$$

we have (as $\Delta \lambda=-e^{2 \lambda} K_{g}$ )

$$
\tilde{\mathscr{L}}_{g}=\Delta+2\langle\nabla \lambda, \nabla \cdot\rangle+\left(|\nabla \lambda|^{2}+3 \Delta \lambda\right),
$$

so $v_{\varepsilon, \delta}^{i}$ solves

$$
\tilde{\mathscr{L}}_{g}^{*} \tilde{\mathscr{L}}_{g} v_{\varepsilon, \delta}^{i}=0,
$$

where one checks that there exists polynomial functions $P_{k, l}: \mathbb{R}^{2} \times \mathbb{R}^{4} \rightarrow \mathrm{M}_{2}(\mathbb{R}), \vec{Q}$ : $\mathbb{R}^{2} \times \mathbb{R}^{4} \rightarrow \mathbb{R}^{2}$ and $R: \mathbb{R}^{2} \times \mathbb{R}^{4} \rightarrow \mathbb{R}$ such that

$$
\tilde{\mathscr{L}}_{g} \mathscr{L}_{g} v_{\varepsilon, \delta}^{i}=\Delta^{2} v_{\varepsilon, \delta}^{i}+\vec{P}\left(\nabla \lambda, \nabla^{2} \lambda\right) \cdot \nabla^{2} v_{\varepsilon, \delta}^{i}+\vec{Q}\left(\nabla \lambda, \nabla^{2} \lambda\right) \cdot \nabla v_{\varepsilon, \delta}^{i}+R\left(\nabla \lambda, \nabla^{2} \lambda\right) v_{\varepsilon, \delta}^{i} .
$$

Therefore, thanks to elliptic regularity and (5.11), we deduce that

$$
\begin{gathered}
\int_{S^{1}}\left(v_{\varepsilon, \delta}^{i} \partial_{\nu}\left(\left(\mathscr{L}_{m}-\Delta\right) v_{\varepsilon, \delta}^{i}\right)-\partial_{\nu} v_{\varepsilon, \delta}^{i}\left(\mathscr{L}_{m}-\Delta\right) v_{\varepsilon, \delta}^{i}\right) d \mathscr{H}^{1} \\
-4(m+1)(m-1) \int_{S^{1}}\left(\left(v_{\varepsilon, \delta}^{i}\right)^{2}-v_{\varepsilon, \delta}^{i} \partial_{\nu}^{2} v_{\varepsilon, \delta}^{i}\right) d \mathscr{H}^{1}
\end{gathered}
$$

is uniformly bounded in $0<\varepsilon<\varepsilon_{0}$ and $0<\delta<\delta(\varepsilon)<\varepsilon$. Furthermore, there exists $C_{0}=C_{0}\left(U_{j}, \vec{\Psi}\right)>0$ such that

$$
\begin{aligned}
& \mid \int_{S^{1}}\left(v_{\varepsilon, \delta}^{i} \partial_{\nu}\left(\left(\mathscr{L}_{m}-\Delta\right) v_{\varepsilon, \delta}^{i}\right)-\partial_{\nu} v_{\varepsilon, \delta}^{i}\left(\mathscr{L}_{m}-\Delta\right) v_{\varepsilon, \delta}^{i}\right) d \mathscr{H}^{1} \\
& \quad-4(m+1)(m-1) \int_{S^{1}}\left(\left(v_{\varepsilon, \delta}^{i}\right)^{2}-v_{\varepsilon, \delta}^{i} \partial_{\nu}^{2} v_{\varepsilon, \delta}^{i}\right) d \mathscr{H}^{1} \mid \leq C_{0} \omega\left(\|v\|_{\mathrm{W}^{2,2}(\Sigma)}\right) .
\end{aligned}
$$

Therefore, we have by (5.17), (5.19) and (5.21)

$$
\begin{aligned}
\int_{B_{1} \backslash \bar{B}_{\delta}(0)}\left(\Delta v_{\varepsilon, \delta}^{i}+(m+1)(m-1) \frac{v_{\varepsilon, \delta}^{i}}{|x|^{2}}\right)^{2} d x \\
+4(m+1)(m-1) \int_{B_{1} \backslash \bar{B}_{\delta}(0)}\left(\frac{x}{|x|^{2}} \cdot \nabla v_{\varepsilon, \delta}^{i}-\frac{v_{\varepsilon, \delta}^{i}}{|x|^{2}}\right)^{2} d x \\
\quad-\frac{1}{\kappa} \frac{1}{(m+1)^{4}} \int_{B_{1} \backslash \bar{B}_{\delta}(0)}\left(2(m+1)^{3} \nabla \zeta \cdot \nabla v_{\varepsilon, \delta}^{i}\right. \\
\left.\quad+\left(-2(m+1) \frac{x}{|x|^{2}} \cdot \nabla \zeta+\left(|\nabla \zeta|^{2}+3 \Delta \zeta\right)\right) v_{\varepsilon, \delta}^{i}\right)^{2} d x \\
\leq \frac{1}{1-\kappa} C_{1} \omega\left(\|v\|_{\mathrm{W}^{2,2}(\Sigma)}\right) .
\end{aligned}
$$

Choose now $\kappa=\frac{1}{4}$, and define

$$
\begin{aligned}
& \zeta_{2}=4(m+1) \zeta \\
& \vec{\zeta}_{3}=\frac{4}{(m+1)} x \cdot \nabla \zeta-\frac{2}{(m+1)^{2}} x\left(|\nabla \zeta|^{2}+3 \Delta \zeta\right),
\end{aligned}
$$


we find

$$
\begin{aligned}
& \int_{B_{1} \backslash \bar{B}_{\delta}(0)}\left(\Delta v_{\varepsilon, \delta}^{i}+(m+1)(m-1) \frac{v_{\varepsilon, \delta}^{i}}{|x|^{2}}\right)^{2} d x \\
& +4(m+1)(m-1) \int_{B_{1} \backslash \bar{B}_{\delta}(0)}\left(\frac{x}{|x|^{2}} \cdot \nabla v_{\varepsilon, \delta}^{i}-\frac{v_{\varepsilon, \delta}^{i}}{|x|^{2}}\right)^{2} d x \\
& -\int_{B_{1} \backslash \bar{B}_{\delta}(0)}\left(\nabla \zeta_{2} \cdot \nabla v_{\varepsilon, \delta}^{i}-\frac{x}{|x|^{2}} \cdot \vec{\zeta}_{3} v_{\varepsilon, \delta}^{i}\right)^{2} d x \leq \frac{4}{3} C_{1} \omega\left(\|v\|_{\mathrm{W}^{2,2}(\Sigma)}\right) .
\end{aligned}
$$

This concludes the proof of the theorem.

\subsection{Indicial roots analysis: case of embedded ends}

The following theorem is the analogous of Theorem VI.1 of [4] and Theorem 1 of [3] from Ginzburg-Landau theory.

Proposition 5.5 Assume that the minimal surface $\vec{\Phi}$ of Theorem A has embedded ends. Then there exists $v_{\varepsilon}^{i} \in C^{\infty}\left(\Sigma \backslash\left(\bar{B}_{\varepsilon}\left(p_{i}\right) \cup\left\{p_{1}, \ldots, p_{n}\right\}\right)\right)$ such that for all compact $K \subset \Sigma_{\varepsilon}^{i}$, we have (up to a subsequence as $\delta \rightarrow 0$ )

$$
v_{\varepsilon, \delta}^{i} \underset{\delta \rightarrow 0}{\longrightarrow} v_{\varepsilon}^{i} \quad \text { in } C^{l}(K) \text { for all } l \in \mathbb{N} .
$$

Furthermore, for all $j \neq i$, we have an expansion in $U_{j}$ as

$$
v_{\varepsilon}^{i}(z)=\operatorname{Re}\left(\gamma_{0} z+\gamma_{1} z^{2}\right)+\gamma_{2}|z|^{2}+\gamma_{3}|z|^{2} \log |z|+\varphi_{\varepsilon}(z)
$$

for some real-analytic function $\varphi_{\varepsilon}$ such that $\varphi_{\varepsilon}(z)=O\left(|z|^{3}\right)$. Therefore, if $u_{\varepsilon}^{i}=|\vec{\Phi}|^{2} v_{\varepsilon}^{i}$, there exists $a_{i, j}, b_{i, j} \in \mathbb{R}$ and $c_{i, j}, d_{i, j} \in \mathbb{C}$ and $\psi_{\varepsilon} \in C^{\infty}(B(0,1) \backslash\{0\})$ such that

$$
u_{\varepsilon}^{i}(z)=\operatorname{Re}\left(\frac{c_{i, j}}{z}+d_{i, j} \frac{\bar{z}}{z}\right)+a_{i, j} \log |z|+b_{i, j}+\psi_{\varepsilon}(z) .
$$

and for all $l \in \mathbb{N}$,

$$
\left|\nabla^{l} \psi_{\varepsilon}(z)\right|=O\left(|z|^{1-l}\right)
$$

Remark 5.6 Although $a_{i, j}, b_{i, j}, c_{i, j}$ and $d_{i, j}$ depends on $\varepsilon$, we remove this explicit dependence for the sake of simplicity of notation.

\section{Proof Step 1: Indicial roots analysis.}

We make computations as previously in the previously fixed chart $\varphi_{j}: U_{j} \rightarrow B(0,1) \subset \mathbb{C}$ such that $\varphi_{j}\left(p_{j}\right)=0$. By [32] (p. 25) the asymptotic expansion of $v_{\varepsilon, \delta}^{i}$ at 0 depends only on the linearised operator of $e^{\lambda} \mathscr{L}_{g}\left(e^{\lambda} \cdot\right)$, which is as $\vec{\Phi}$ has embedded ends

$$
\mathscr{L}=\Delta-4 \frac{x}{|x|^{2}} \cdot \nabla+\frac{4}{|x|^{2}} .
$$

Therefore, without loss of generality, we can assume that $\mathscr{L}^{*} \mathscr{L} v_{\varepsilon, \delta}^{i}=0$. Taking polar coordinates $(r, \theta)$ centred at the origin, recall that

$$
\Delta=\partial_{r}^{2}+\frac{1}{r} \partial_{r}+\frac{1}{r^{2}} \partial_{\theta}^{2} .
$$


Therefore, we have

$$
\mathscr{L}=\partial_{r}^{2}+\frac{1}{r} \partial_{r}+\frac{1}{r^{2}} \partial_{\theta}^{2}-4 \frac{1}{r} \partial_{r}+\frac{4}{r^{2}}=\partial_{r}^{2}-\frac{3}{r} \partial_{r}+\frac{4}{r^{2}}+\frac{1}{r^{2}} \partial_{\theta}^{2},
$$

and

$$
\mathscr{L}^{*}=\partial_{r}^{2}+\frac{5}{r} \partial_{r}+\frac{4}{r^{2}}+\frac{1}{r^{2}} \partial_{\theta}^{2} .
$$

Projecting to $\operatorname{Vect}\left(e^{i k \cdot}\right)$ (where $k \in \mathbb{Z}$ is fixed), the operator $\mathscr{L}$ (resp. $\mathscr{L}^{*}$ ) becomes

$$
\mathscr{L}_{k}=\partial_{r}^{2}-\frac{3}{r} \partial_{r}+\frac{4-k^{2}}{r^{2}} \quad\left(\text { resp. } \mathscr{L}_{k}^{*}=\partial_{r}^{2}+\frac{5}{r} \partial_{r}+\frac{4-k^{2}}{r^{2}}\right)
$$

and we define for all $k \in \mathbb{Z}$ the functions $v_{\varepsilon, \delta}^{i}(k, \cdot):(\delta, 1) \rightarrow \mathbb{C}$ by

$$
v_{\varepsilon, \delta}^{i}(r, \theta)=\sum_{k \in \mathbb{Z}} v_{\varepsilon, \delta}^{i}(k, r) e^{i k \theta} .
$$

As $\mathscr{L}_{k}^{*} \mathscr{L}_{k} v_{\varepsilon, \delta}^{i}(k, \cdot)=0$, and the space of solutions to $\mathscr{L}_{k}^{*} \mathscr{L}_{k} u=0$ is four-dimensional, we only need to find a basis of solutions to $\mathscr{L}_{k}^{*} \mathscr{L}_{k} u=0$ to obtain all possible asymptotic behaviour at the origin.

Let $\alpha \in \mathbb{C}$ fixed, we have

$$
\begin{aligned}
& \mathscr{L}_{k} r^{\alpha}=\alpha(\alpha-1) r^{\alpha-2}-3 \alpha r^{\alpha-2}+\left(4-k^{2}\right) r^{\alpha-2}=\left(\alpha^{2}-4 \alpha+4-k^{2}\right) r^{\alpha-2} \\
& \mathscr{L}_{k}^{*} \mathscr{L}_{k} r^{\alpha}=\left(\alpha^{2}-k^{2}\right)\left(\alpha^{2}-4 \alpha+4-k^{2}\right) r^{\alpha-4},
\end{aligned}
$$

so the basis of solutions to $\mathscr{L}^{*} \mathscr{L}_{k} u=0$ is given by

$$
u_{1}^{ \pm}=r^{\alpha_{k}^{ \pm}}, \quad u_{2}^{ \pm}=r^{\beta_{k}^{ \pm}}
$$

where

$$
\alpha_{k}^{ \pm}=2 \pm|k|, \quad \beta_{k}^{ \pm}= \pm|k| .
$$

In particular, for $k=0$, we need to find two other solutions. For $k=0$, we have

$$
\mathscr{L}_{0}^{*} \mathscr{L}_{0}=\partial_{r}^{4}+\frac{2}{r} \partial_{r}^{3}-\frac{1}{r^{2}} \partial_{r}^{2}+\frac{1}{r^{3}} \partial_{r}
$$

so one easily check that a basis of solutions of $\mathscr{L}^{*} \mathscr{L} u=0$ is given by

$$
1, r^{2}, \log (r), r^{2} \log (r)
$$

and that furthermore,

$$
\mathscr{L}_{0}\left(r^{2}\right)=\mathscr{L}_{0}\left(r^{2} \log (r)\right)=0 .
$$

Finally, for $|k|=1$, as $\left\{\alpha_{k}^{+}, \alpha_{k}^{-}\right\}=\{1,3\}$ and $\left\{\beta_{k}^{+}, \beta_{k}^{-}\right\}=\{-1,1\}$, we only have three solutions and we need to find an additional one. As $\operatorname{Ker}\left(\mathscr{L}_{1}^{*}\right)=\left\{r^{-3}, r^{-1}\right\}$, we need to find a solution $u$ such that $\mathscr{L} u \neq 0, \mathscr{L} u \in \operatorname{Ker}\left(\mathscr{L}_{1}^{*}\right)$ and $u \notin \operatorname{Span}_{\mathbb{R}}\left(r^{-1}, r, r^{3}\right)$. One checks directly that this additional solution is given by

$$
u(r)=r \log (r),
$$

which satisfies indeed

$$
\mathscr{L} u=\frac{1}{r}-\frac{3}{r}(\log (r)+1)+\frac{3}{r^{2}}(r \log (r))=-\frac{2}{r} \in \operatorname{Ker}\left(\mathscr{L}^{*}\right) .
$$


Notice that these computations also show that $\mathscr{L}^{*} \mathscr{L}=\Delta^{2}$, but we did not want to use this result directly to obtain a formally similar proof in the case of ends of higher multiplicity.

Step 2: Estimate on the biharmonic components.

Now, recall that

$$
\begin{aligned}
& \int_{B_{1} \backslash \bar{B}_{\delta}(0)}\left(\Delta v_{\varepsilon, \delta}^{i}-2(m+1)\left(\frac{x}{|x|^{2}}+\nabla \zeta_{0}\right) \cdot \nabla v_{\varepsilon, \delta}^{i}+\frac{(m+1)^{2}}{|x|^{2}}\left(1+x \cdot \vec{\zeta}_{1}\right) v_{\varepsilon, \delta}^{i}\right)^{2} d x \\
& \quad \leq C \omega\left(\|v\|_{\mathrm{W}^{2,2}(\Sigma)}\right) .
\end{aligned}
$$

Now, let $\gamma, \gamma_{k}^{1}, \gamma_{k}^{2}, \gamma_{k}^{3}, \gamma_{k}^{4} \in \mathbb{C}$ (for $k \in \mathbb{Z}$ ) be such that

$$
\begin{aligned}
v_{\varepsilon, \delta}^{i}(r, \theta)= & \sum_{k \in \mathbb{Z}^{*}}\left(\gamma_{k}^{1} r^{2+k}+\gamma_{k}^{2} r^{2-k}+\gamma_{k}^{3} r^{k}+\gamma_{k}^{4} r^{-k}\right) e^{i k \theta}+\left(\gamma r e^{i \theta}+\bar{\gamma} r e^{-i \theta}\right) \log (r) \\
& +\gamma_{0}^{1}+\gamma_{0}^{2} \log (r)+\gamma_{0}^{3} r^{2}+\gamma_{0}^{4} r^{2} \log (r) .
\end{aligned}
$$

As $v_{\varepsilon, \delta}^{i}$ is real, we have for all $k \in \mathbb{Z}$

$$
\gamma_{-k}^{2}=\overline{\gamma_{k}^{1}} \quad \text { and } \gamma_{-k}^{4}=\overline{\gamma_{k}^{3}}
$$

Now, we have

$$
\begin{aligned}
\mathscr{L} v_{\varepsilon, \delta}^{i}= & 4 \sum_{k \in \mathbb{Z}^{*}}\left(-(k-1) \gamma_{k}^{3} r^{k-2}+(k+1) \gamma_{k}^{4} r^{-k-2}\right) e^{i k \theta} \\
& -2\left(\frac{\gamma}{r} e^{i \theta}+\frac{\bar{\gamma}}{r} e^{-i \theta}\right)+\frac{4 \gamma_{0}^{1}}{r^{2}}+\frac{4 \gamma_{0}^{2}}{r^{2}}(\log (r)-1) .
\end{aligned}
$$

Therefore, as

$$
\tilde{\mathscr{L}}_{g}=\mathscr{L}-4 \nabla \zeta_{0} \cdot \nabla+(m+1)^{2} \frac{x}{|x|^{2}} \cdot \vec{\zeta}_{1}
$$

for two real-analytic functions $\zeta_{0}: B(0,1) \rightarrow \mathbb{R}$ and $\vec{\zeta}_{1}: B(0,1) \rightarrow \mathbb{R}$, we deduce from (5.24) that

$$
\begin{aligned}
\tilde{\mathscr{L}}_{g} v_{\varepsilon, \delta}^{i}= & 4 \sum_{k \in \mathbb{Z} \backslash\{0,1\}}-(k-1) \gamma_{k}^{3} r^{k-2}(1+O(r)) e^{i k \theta} \\
& +4 \sum_{k \in \mathbb{Z} \backslash\{-1,0\}}(k+1) \gamma_{k}^{4} r^{-k-2}(1+O(r)) e^{i k \theta} \\
& -2\left(\frac{\gamma}{r}(1+O(r)) e^{i \theta}+\frac{\bar{\gamma}}{r}(1+O(r)) e^{-i \theta}\right) \\
& +\frac{4\left(\gamma_{0}^{1}-\gamma_{0}^{2}\right)}{r^{2}}(1+O(r))+\frac{4 \gamma_{0}^{1}}{r^{2}} \log (r)(1+O(r)) .
\end{aligned}
$$

Notice that the first two sums do not involve powers in $1 / r$ (this justifies why there are no cross terms between these two sums and the remaining terms). Now fix some $0<R<1$ such that the " $O(1)$ functions" be bounded by $1 / 2$ (in absolute value) on $B_{R} \backslash \bar{B}_{\delta}(0)$. Then we have by Parseval identity

$$
\int_{B_{R} \backslash \bar{B}_{\delta}(0)}\left(\tilde{\mathscr{L}}_{g} v_{\varepsilon, \delta}^{i}\right)^{2} d x=32 \pi \sum_{k \in \mathbb{Z}^{*}} \int_{\delta}^{R}\left((k-1)^{2}\left|\gamma_{k}^{3}\right|^{2} r^{2(k-2)}+(k+1)^{2}\left|\gamma_{k}^{4}\right|^{2} r^{-2(k+2)}\right.
$$




$$
\begin{aligned}
& \left.+2(k+1)(k-1) \operatorname{Re}\left(\gamma_{k}^{3} \overline{\gamma_{k}^{4}}\right) r^{-4}\right)(1+O(r)) r d r+16 \pi \int_{\delta}^{R}\left(\frac{|\gamma|^{2}}{r^{2}}(1+O(r))\right) r d r \\
& +32 \pi \int_{\delta}^{R}\left(\frac{\left(\gamma_{0}^{1}-\gamma_{0}^{2}\right)^{2}}{r^{4}}+\frac{\left(\gamma_{0}^{1}\right)^{2}}{r^{4}} \log ^{2}(r)+2\left(\gamma_{0}^{1}-\gamma_{0}^{2}\right) \gamma_{0}^{1} \frac{\log (r)}{r^{4}}\right)(1+O(r)) r d r \\
& =16 \pi \sum_{k \in \mathbb{Z} \backslash\{0,1\}}(k-1)\left|\gamma_{k}^{3}\right|^{2}\left(R^{2(k-1)}(1+O(R))-\delta^{2(k-1)}(1+O(\delta))\right) \\
& +16 \pi \sum_{k \in \mathbb{Z} \backslash\{-1,0\}}-(k+1)\left|\gamma_{k}^{4}\right|^{2}\left(R^{-2(k+1)}(1+O(R))-\delta^{-2(k+1)}(1+O(\delta))\right) \\
& +16 \pi\left(\gamma_{0}^{1}-\gamma_{0}^{2}\right)^{2}\left(\frac{1}{\delta^{2}}(1+O(\delta))-\frac{1}{R^{2}}(1+O(R))\right) \\
& +8 \pi\left(\gamma_{0}^{1}\right)^{2}\left(\frac{1}{\delta^{2}}(1+O(\delta))\left(2 \log ^{2}(\delta)+2 \log (\delta)+1\right)\right. \\
& \left.-\frac{1}{R^{2}}(1+O(R))\left(\log ^{2}(R)+2 \log (R)+1\right)\right) \\
& +8 \pi \gamma_{0}^{1}\left(\gamma_{0}^{1}-\gamma_{0}^{2}\right)\left(\frac{1}{\delta^{2}}(1+O(\delta))(2 \log (\delta)+1)-\frac{1}{R^{2}}(1+O(R))(2 \log (R)+1)\right) \\
& +32 \pi \sum_{k \in \mathbb{Z}^{*}} \operatorname{Re}\left(\gamma_{k}^{3} \overline{\gamma_{k}^{4}}\right)\left(\frac{1}{\delta^{2}}(1+O(\delta))-\frac{1}{R^{2}}(1+O(R))\right) \\
& =16 \pi \sum_{k \geq 2}(|k|-1)\left|\gamma_{k}^{3}\right|^{2} R^{2(|k|-1)}\left(1+O(R)-\left(\frac{\delta}{R}\right)^{2(|k|-1)}(1+O(\delta))\right) \\
& +16 \pi \sum_{k \leq-1}(|k|+1)\left|\gamma_{k}^{3}\right|^{2} \frac{1}{\delta^{2(|k|+1)}}\left(1+O(\delta)-\left(\frac{\delta}{R}\right)^{2(|k|+1)}(1+O(R))\right) \\
& +16 \pi \sum_{k \geq 1}(|k|+1)\left|\gamma_{k}^{4}\right|^{2} \frac{1}{\delta^{2(|k|+1)}}\left(1+O(\delta)-\left(\frac{\delta}{R}\right)^{2(|k|+1)}(1+O(R))\right) \\
& +16 \pi \sum_{k \geq-2}(|k|-1)\left|\gamma_{k}^{4}\right|^{2} R^{2(|k|-1)}\left(1+O(R)-\left(\frac{\delta}{R}\right)^{2(|k|-1)}(1+O(\delta))\right) \\
& +16 \pi|\gamma|^{2}\left(\log \left(\frac{1}{\delta}\right)(1+O(\delta))+\log (R)(1+O(R))\right) \\
& +16 \pi\left(\gamma_{0}^{1}-\gamma_{0}^{2}\right)^{2} \frac{1}{\delta^{2}}\left(1+O(\delta)-\left(\frac{\delta}{R}\right)(1+O(R))\right) \\
& +8 \pi\left(\gamma_{0}^{1}\right)^{2}\left(\frac{1}{\delta^{2}}(1+O(\delta))\left(2 \log ^{2}(\delta)+2 \log (\delta)+1\right)\right. \\
& \left.-\frac{1}{R^{2}}(1+O(R))\left(\log ^{2}(R)+2 \log (R)+1\right)\right) \\
& +8 \pi \gamma_{0}^{1}\left(\gamma_{0}^{1}-\gamma_{0}^{2}\right)\left(\frac{1}{\delta^{2}}(1+O(\delta))(2 \log (\delta)+1)-\frac{1}{R^{2}}(1+O(R))(2 \log (R)+1)\right)
\end{aligned}
$$




$$
+32 \pi \sum_{k \in \mathbb{Z}^{*}}(k+1)(k-1) \operatorname{Re}\left(\gamma_{k}^{3} \overline{\gamma_{k}^{4}}\right) \frac{1}{\delta^{2}}\left(1+O(\delta)-\left(\frac{\delta}{R}\right)^{2}(1+O(R))\right) .
$$

As the quantity in the left-hand side of (5.25) is bounded independently of $0<\delta<R$, we deduce that for all $k \geq 1$, and some uniform constant $C>0$

$$
\begin{aligned}
& \left|\gamma_{-|k|}^{3}\right| \leq C \delta^{|k|+1} \underset{\delta \rightarrow 0}{\longrightarrow} 0 \\
& \left|\gamma_{|k|}^{4}\right| \leq C \delta^{|k|+1} \underset{\delta \rightarrow 0}{\longrightarrow} 0 .
\end{aligned}
$$

Notice that the second estimate follows from the first one as $\gamma_{k}^{4}=\overline{\gamma_{-k}^{3}}$. Furthermore, as

$$
\sum_{k \geq 1}(|k|+1)\left|\gamma_{-k}^{3}\right|^{2} \frac{1}{\delta^{2(|k|+1)}}<\infty
$$

and is bounded independently of $\delta>0$, there exists $C>0$ such that

$$
\left|\gamma_{-k}^{3}\right| \leq \frac{C}{|k|+1} \delta^{|k|+1}
$$

Now, we see the next order of singularity is given by $\log ^{2}(\delta) / \delta^{2}$, so we have

$$
\left|\gamma_{0}^{1}\right| \leq C \frac{\delta}{\log \left(\frac{1}{\delta}\right)} \underset{\delta \rightarrow 0}{\longrightarrow} 0 .
$$

Another singular term is

$$
\frac{1}{\delta^{2}} \sum_{k \in \mathbb{Z} \backslash\{-1,0,1\}}(k+1)(k-1) \operatorname{Re}\left(\gamma_{k}^{3} \overline{\gamma_{k}^{4}}\right),
$$

but (5.26) implies that (as the $\gamma_{k}$ are also uniformly bounded)

$$
\begin{aligned}
& \frac{1}{\delta^{2}}\left|\sum_{k \in \mathbb{Z} \backslash\{-1,0,1\}}(k+1)(k-1) \operatorname{Re}\left(\gamma_{k}^{3} \overline{\gamma_{k}^{4}}\right)\right| \leq \frac{C}{\delta^{2}} \sum_{k \geq 2}(|k|+1)(|k|-1) \delta^{|k|+1} \\
& \quad \leq C \sum_{|k| \geq 2}(|k|+1)|k| \delta^{|k|-1}=C\left(\frac{3 \delta}{1-\delta}-\frac{6 \delta^{2}}{(1-\delta)}+\frac{2 \delta^{3}}{(1-\delta)^{3}}\right) \leq 4 C \delta \underset{\delta \rightarrow 0}{\longrightarrow} 0 .
\end{aligned}
$$

for $0<\delta<1$ small enough. The next singular term is

$$
16 \pi\left(\gamma_{0}^{1}-\gamma_{0}^{2}\right)^{2} \frac{1}{\delta^{2}}\left(1+O(\delta)-\left(\frac{\delta}{R}\right)(1+O(R))\right),
$$

and using (5.28), we deduce that

$$
\left(\gamma_{0}^{1}-\gamma_{0}^{2}\right)^{2} \frac{1}{\delta^{2}}=\frac{\left|\gamma_{0}^{2}\right|^{2}}{\delta^{2}}+O\left(\frac{1}{\log ^{2}\left(\frac{1}{\delta}\right)}\right)
$$

so we deduce that

$$
\left|\gamma_{0}^{2}\right| \leq C \delta \underset{\delta \rightarrow 0}{\longrightarrow} 0
$$


Finally, the last singular term is

$$
16 \pi|\gamma|^{2} \log \left(\frac{1}{\delta}\right)
$$

and we deduce that

$$
|\gamma| \leq C \sqrt{\frac{1}{\log \left(\frac{1}{\delta}\right)}} \underset{\delta \rightarrow 0}{\longrightarrow} 0 .
$$

Step 3: Estimates on the harmonic components. Now, we have the inequality (from Theorem 5.4)

$$
\int_{B_{R} \backslash \bar{B}_{\delta}(0)}\left(\Delta v_{\varepsilon, \delta}^{i}\right)^{2} d x-\int_{B_{R} \backslash \bar{B}_{\delta}(0)}\left(\nabla \zeta_{2} \cdot \nabla v_{\varepsilon, \delta}^{i}-\frac{x}{|x|^{2}} \cdot \vec{\zeta}_{3} v_{\varepsilon, \delta}^{i}\right)^{2} d x \leq C \omega\left(\|v\|_{\mathrm{W}^{2,2}(\Sigma)}\right) .
$$

Thanks to (5.23)

$$
\begin{aligned}
\Delta v_{\varepsilon, \delta}^{i}= & 4 \sum_{k \in \mathbb{Z}^{*}}\left((k+1) \gamma_{k}^{1} r^{k}-(k-1) \gamma_{k}^{2} r^{-k}\right) e^{i k \theta} \\
& +2\left(\frac{\gamma}{r} e^{i \theta}+\frac{\bar{\gamma}}{r} e^{-i \theta}\right)+4 \gamma_{0}^{3}+4 \gamma_{0}^{4}(\log r+1) .
\end{aligned}
$$

Furthermore, we have

$$
\begin{aligned}
\nabla \zeta_{2} & \cdot \nabla v_{\varepsilon, \delta}^{i}-\frac{x}{|x|^{2}} \cdot \vec{\zeta}_{3} v_{\varepsilon, \delta}^{i} \\
= & \sum_{k \in \mathbb{Z}^{*}}\left(\gamma_{k}^{1} O\left(r^{1+k}\right)+\gamma_{k}^{2} O\left(r^{1-k}\right)+\gamma_{k}^{3} O\left(r^{k-1}\right)+\gamma_{k}^{4} O\left(r^{-k-1}\right)\right) e^{i k \theta} \\
& +\gamma O(1)+\gamma_{0}^{1} O\left(\frac{1}{r}\right)+\gamma_{0}^{2} \log (r) O\left(\frac{1}{r}\right)+\gamma_{0}^{3} O(r)+\gamma_{0}^{4} \log r O(r) .
\end{aligned}
$$

Therefore, we have

$$
\begin{aligned}
& \int_{B_{R} \backslash \bar{B}_{\delta}(0)}\left(\Delta v_{\varepsilon, \delta}^{i}\right)^{2} d x=32 \pi \sum_{k \in \mathbb{Z}^{*}} \int_{\delta}^{R}\left((k+1)^{2}\left|\gamma_{k}^{1}\right|^{2} r^{2 k}+(k-1)^{2}\left|\gamma_{k}^{2}\right|^{2} r^{-2 k}\right. \\
& \left.-2(k+1)(k-1) \operatorname{Re}\left(\gamma_{k}^{1} \overline{\gamma_{k}^{2}}\right)\right) r d r+16 \pi \int_{\delta}^{R}\left(\frac{|\gamma|^{2}}{r^{2}}(1+O(r))\right) r d r \\
& +32 \pi \int_{\delta}^{R}\left(\left|\gamma_{0}^{3}+\gamma_{0}^{4}\right|^{2}+\left|\gamma_{0}^{4}\right|^{2} \log ^{2}(r)+2\left(\gamma_{0}^{3}+\gamma_{0}^{4}\right) \gamma_{0}^{4} \log (r)\right) r d r
\end{aligned}
$$

Notice that the second integral involving the square of the radial component of $\Delta v_{\varepsilon, \delta}^{i}$ is bounded, so we can neglect this term. Now, we also have as $|a+b+c+d|^{2} \leq$ $4\left(|a|^{2}+|b|^{2}+|c|^{2}+|d|^{2}\right)$ for all $a, b, c, d \in \mathbb{C}$ and by Parseval identity

$$
\begin{aligned}
& \int_{B_{R} \backslash \bar{B}_{\delta}(0)}\left(\nabla \zeta_{2} \cdot \nabla v_{\varepsilon, \delta}^{i}-\frac{x}{|x|^{2}} \cdot \vec{\zeta}_{3} v_{\varepsilon, \delta}^{i}\right)^{2} d x \\
& \quad \leq 8 \pi \sum_{k \in \mathbb{Z}^{*}} \int_{\delta}^{R}\left(\left|\gamma_{k}^{1}\right|^{2} O\left(r^{2+2 k}\right)+\left|\gamma_{k}^{2}\right|^{2} O\left(r^{2-2 k}\right)+\left|\gamma_{k}^{3}\right|^{2} O\left(r^{2 k-2}\right)+\left|\gamma_{k}^{4}\right|^{2} O\left(r^{-2 k-2}\right)\right) r d r
\end{aligned}
$$




$$
\begin{aligned}
& +8 \pi \int_{\delta}^{R}\left(\left|\gamma_{0}^{1}\right|^{2} O\left(\frac{1}{r^{2}}\right)+\left|\gamma_{0}^{2}\right|^{2} O\left(\frac{\log ^{2}(r)}{r^{2}}\right)+\left|\gamma_{0}^{3}\right|^{2} O\left(r^{2}\right)\right. \\
& \left.+\left|\gamma_{0}^{4}\right|^{2} O\left(r^{2} \log ^{2}(r)\right)\right) r d r+|\gamma| O(R) .
\end{aligned}
$$

Now notice that

$$
\sum_{k \geq 1} \int_{\delta}^{R}\left(\left|\gamma_{k}^{3}\right|^{2} O\left(r^{2 k-2}\right)+\left|\gamma_{-k}^{4}\right|^{2} O\left(r^{2 k-2}\right)\right) r d r
$$

is bounded in $\delta$, and (5.27) imply that

$$
\begin{aligned}
& \left|\sum_{k \geq 1}\left(\left|\gamma_{-k}^{3}\right|^{2} O\left(r^{-2 k-2}\right)+\left|\gamma_{k}^{4}\right| O\left(r^{-2 k-2}\right)\right) r d r\right| \leq C \sum_{k \geq 1} \int_{\delta}^{R} \frac{\delta^{2 k+2}}{(|k|+1)^{2}} r^{-2 k-1} d r \\
& \quad=C \sum_{k \geq 1} \frac{\delta^{2}}{2 k(k+1)^{2}}\left(1-\left(\frac{\delta}{R}\right)^{2 k}\right) \leq C\left(1-\frac{\pi^{2}}{12}\right) \delta^{2} \underset{\delta \rightarrow 0}{\longrightarrow} 0
\end{aligned}
$$

where we used

$$
\begin{aligned}
\sum_{k=1}^{\infty} \frac{1}{k(k+1)^{2}} & =\sum_{k=1}^{\infty}\left(\frac{1}{k}-\frac{1}{k+1}\right)-\sum_{k=1}^{\infty} \frac{1}{(k+1)^{2}} \\
& =1-(\zeta(2)-1)=2-\zeta(2)=2-\frac{\pi^{2}}{6}
\end{aligned}
$$

Finally, by (5.33), (5.34) and (5.35), we deduce that there exists $C>0$ (independent of $\delta$ and $\varepsilon$ ) such that

$$
\begin{aligned}
& \mid 8 \pi \sum_{k \in \mathbb{Z}^{*}} \int_{\delta}^{R}\left(\left|\gamma_{k}^{1}\right|^{2} O\left(r^{2+2 k}\right)+\left|\gamma_{k}^{2}\right|^{2} O\left(r^{2-2 k}\right)+\left|\gamma_{k}^{3}\right|^{2} O\left(r^{2 k-2}\right)+\left|\gamma_{k}^{4}\right|^{2} O\left(r^{-2 k-2}\right)\right) r d r \\
& \quad+8 \pi \int_{\delta}^{R}\left(\left|\gamma_{0}^{1}\right|^{2} O\left(\frac{1}{r^{2}}+\left|\gamma_{0}^{2}\right|^{2} O\left(\frac{\log ^{2}(r)}{r^{2}}\right)\right)+\left|\gamma_{0}^{3}\right|^{2} O\left(r^{2}\right)+\left|\gamma_{0}^{4}\right|^{2} O\left(r^{2} \log ^{2}(r)\right)\right) r d r \\
& \quad-8 \pi \sum_{k \in \mathbb{Z}^{*}} \int_{\delta}^{R}\left(\left|\gamma_{k}^{1}\right|^{2} O\left(r^{2+2 k}\right)+\left|\gamma_{k}^{2}\right|^{2} O\left(r^{2-2 k}\right)\right) r d r \mid \leq C
\end{aligned}
$$

Therefore, by (5.32), (5.33), (5.36), and (5.30) (for the term in $|\gamma|^{2} \log (1 / \delta)^{-1}$ ) we have

$$
\begin{aligned}
& \int_{B_{R} \backslash \bar{B}_{\delta}(0)}\left(\Delta v_{\varepsilon, \delta}^{i}\right)^{2} d x-\int_{B_{R} \backslash \bar{B}_{\delta}(0)}\left(\nabla \zeta_{2} \cdot \nabla v_{\varepsilon, \delta}^{i}-\frac{x}{|x|^{2}} \cdot \nabla \vec{\zeta}_{3} v_{\varepsilon, \delta}^{i}\right)^{2} d x \\
& \geq 32 \pi \sum_{k \in \mathbb{Z}^{*}} \int_{\delta}^{R}\left((k+1)^{2}\left|\gamma_{k}^{1}\right|^{2} r^{2 k}\left(1+O\left(r^{2}\right)\right)+(k-1)^{2}\left|\gamma_{k}^{2}\right|^{2} r^{-2 k}\left(1+O\left(r^{2}\right)\right)\right. \\
& \left.\quad-2(k+1)(k-1) \operatorname{Re}\left(\gamma_{k}^{1} \overline{\gamma_{k}^{2}}\right)\right) r d r \\
& \quad+8 \pi \int_{\delta}^{R}\left(\left|\gamma_{-1}^{1}\right|^{2} O(1)+\left|\gamma_{1}^{2}\right|^{2} O(1)\right) r d r \\
& \quad+32 \pi \int_{\delta}^{R}\left(\left|\gamma_{0}^{3}+\gamma_{0}^{4}\right|^{2}+\left|\gamma_{0}^{4}\right|^{2} \log ^{2}(r)+2\left(\gamma_{0}^{3}+\gamma_{0}^{4}\right) \gamma_{0}^{4} \log (r)\right) r d r-C
\end{aligned}
$$


As previously, the terms involving positive powers of $k$ are bounded, and

$$
\begin{aligned}
& \sum_{k \leq-2} \int_{\delta}^{R}(k+1)^{2}\left|\gamma_{k}^{1}\right|^{2} r^{2 k}\left(1+O\left(r^{2}\right)\right) r d r \\
& \quad=\frac{1}{2} \sum_{k \leq-2}(|k|-1)\left|\gamma_{k}^{1}\right|^{2} \frac{1}{\delta^{2(|k|-1)}}\left(1+O\left(\delta^{2}\right)-\left(\frac{\delta}{R}\right)^{2(|k|-1)}\left(1+O\left(R^{2}\right)\right)\right),
\end{aligned}
$$

so for all $k \geq 2$, as (5.31) implies that (5.37) is bounded independently of $\delta$ (and $\varepsilon$ ), we deduce that for some universal constant $C$ (independent of $0<\varepsilon<\varepsilon_{0}$ and $0<\delta<\delta(\varepsilon)<\varepsilon$ )

$$
\left|\gamma_{-|k|}^{1}\right| \leq C \delta^{|k|-1} \underset{\delta \rightarrow 0}{\longrightarrow} 0
$$

Since $\gamma_{-k}^{2}=\overline{\gamma_{k}^{1}}$, we also have for all $k \geq 2$

$$
\left|\gamma_{k}^{2}\right| \leq C \delta^{|k|-1} \underset{\delta \rightarrow 0}{\longrightarrow} 0
$$

Step 4: Conclusion and limit as $\delta \rightarrow 0$.

Finally, we deduce from the two previous steps that

$$
\begin{aligned}
v_{\varepsilon, \delta}^{i}= & \left(\gamma_{1}^{2}+\gamma_{1}^{3}\right) r e^{i \theta}+\left(\gamma_{-1}^{1}+\gamma_{-1}^{4}\right) r e^{-i \theta}+\gamma_{0}^{3} r^{2}+\gamma_{0}^{4} r^{2} \log (r) \\
& +\gamma_{1}^{1} r^{3} e^{3 i \theta}+\gamma_{-1}^{2} r^{3} e^{-3 i \theta}+\sum_{k \geq 2}\left(\left(\gamma_{k}^{1} r^{2+k}+\gamma_{k}^{3} r^{k}\right) e^{i k \theta}\right. \\
& \left.+\left(\gamma_{-k}^{2} r^{2+k}+\gamma_{-k}^{4} r^{k}\right) e^{-i k \theta}\right) \\
& +\sum_{k \leq-2}\left(\left(\gamma_{k}^{2} r^{2-|k|}+\gamma_{k}^{4} r^{-|k|}\right) e^{i k \theta}+\left(\gamma_{-k}^{1} r^{2-|k|}+\gamma_{-k}^{3} r^{-|k|}\right) e^{-i k \theta}\right) \\
& +\gamma_{0}^{1}+\gamma_{0}^{2} \log (r)+\left(\gamma e^{i \theta}+\bar{\gamma} e^{-i \theta}\right) r \log (r) .
\end{aligned}
$$

Thanks if the previous estimates, all coefficients are bounded, and for all fixed $(r, \theta) \in$ $B_{R} \backslash \bar{B}_{\delta}(0)$,

$$
\begin{aligned}
& \mid \sum_{k \leq-2}\left(\left(\gamma_{k}^{2} r^{2-|k|}+\gamma_{k}^{4} r^{-|k|}\right) e^{i k \theta}+\left(\gamma_{-k}^{1} r^{2-|k|}+\gamma_{-k}^{3} r^{-|k|}\right) e^{-i k \theta}\right) \\
& \quad+\gamma_{0}^{1}+\gamma_{0}^{2} \log (r)+\left(\gamma e^{i \theta}+\bar{\gamma} e^{-i \theta}\right) r \log (r) \mid \underset{\delta \rightarrow 0}{\longrightarrow} 0 .
\end{aligned}
$$

Furthermore, as the operator $\widetilde{\mathscr{L}}_{g}^{*} \widetilde{\mathscr{L}}_{g}$ is uniformly elliptic on $\Sigma_{\varepsilon}$ for all fixed $0<\varepsilon<\varepsilon_{0}$ and thanks to the uniform bound, we deduce that up to a subsequence, there exists $v_{\varepsilon}^{i} \in$ $C^{\infty}\left(\Sigma \backslash\left(\bar{B}_{\varepsilon}\left(p_{i}\right) \cup\left\{p_{1}, \ldots, p_{n}\right\}\right)\right)$ such that for all compact $\left.K \subset \Sigma \backslash\left(\bar{B}_{\varepsilon}\left(p_{i}\right) \cup\left\{p_{1}, \ldots, p_{n}\right\}\right)\right)$,

$$
v_{\varepsilon, \delta}^{i} \underset{\delta \rightarrow 0}{\longrightarrow} v_{\varepsilon}^{i} \quad \text { in } C^{l}(K) \text { for all } l \in \mathbb{N} .
$$

Furthermore, as $\delta \rightarrow 0$, (5.38) implies that

$$
\begin{aligned}
v_{\varepsilon}^{i}= & \left(\gamma_{1}^{2}+\gamma_{1}^{3}\right) r e^{i \theta}+\left(\gamma_{-1}^{1}+\gamma_{-1}^{4}\right) r e^{-i \theta}+\gamma_{0}^{3} r^{2}+\gamma_{0}^{4} r^{2} \log (r) \\
& +\gamma_{1}^{1} r^{3} e^{3 i \theta}+\gamma_{-1}^{2} r^{3} e^{-3 i \theta}+\sum_{k \geq 2}\left(\left(\gamma_{k}^{1} r^{2+k}+\gamma_{k}^{3} r^{k}\right) e^{i k \theta}+\left(\gamma_{-k}^{2} r^{2+k}+\gamma_{-k}^{4} r^{k}\right) e^{-i k \theta}\right)
\end{aligned}
$$




$$
\begin{aligned}
& =\operatorname{Re}\left(\gamma_{0} z+\gamma_{1} z^{2}\right)+\gamma_{2}|z|^{2}+\gamma_{3}|z|^{2} \log |z|+\varphi(z), \\
& =\operatorname{Re}\left(\gamma_{0} z+\gamma_{1} z^{2}\right)+\gamma_{2}|z|^{2}+\gamma_{3}|z|^{2} \log |z|+O\left(|z|^{3}\right),
\end{aligned}
$$

where $\varphi$ is real analytic and $\varphi(z)=O\left(|z|^{3}\right)$. Finally, by the Weierstrass parametrisation, if $\vec{\Phi}$ has embedded ends, we can assume [39] that up to rotation $\vec{\Phi}$ admits the following expansion for some $\alpha>0$ and $\beta \in \mathbb{R}$

$$
\vec{\Phi}(z)=\operatorname{Re}\left(\frac{\alpha_{j}}{z}+O(|z|), \frac{i \alpha_{j}}{z}+O(|z|), \beta \log |z|\right) .
$$

Therefore, we have

$$
\partial_{z} \vec{\Phi}(z)=\frac{1}{2}\left(-\frac{\alpha_{j}}{z^{2}}+O(1),-\frac{i \alpha_{j}}{z^{2}}+O(1), \frac{\beta}{z}+O(1)\right)
$$

and

$$
e^{2 \lambda}=2\left|\partial_{z} \vec{\Phi}\right|^{2}=\frac{\alpha_{j}^{2}}{|z|^{4}}+O\left(\frac{1}{|z|^{2}}\right)=\frac{\alpha_{j}^{2}}{|z|^{4}}\left(1+O\left(|z|^{2}\right)\right) .
$$

Therefore, we have

$$
\begin{aligned}
u_{\varepsilon}^{i} & =e^{\lambda} v_{\varepsilon, \delta}^{i}=\frac{\alpha_{j}}{|z|^{2}}\left(1+O\left(|z|^{2}\right)\right)\left(\operatorname{Re}\left(\gamma_{0} z+\gamma_{1} z^{2}\right)+\gamma_{2}|z|^{2}+\gamma_{3}|z|^{2} \log |z|+O\left(|z|^{3}\right)\right) \\
& =\operatorname{Re}\left(\frac{\alpha_{j} \overline{\gamma_{0}}}{z}+\alpha_{j} \overline{\gamma_{1}} \frac{\bar{z}}{z}\right)+\alpha_{j} \gamma_{2}+\alpha_{j} \gamma_{3} \log |z|+O(|z|)
\end{aligned}
$$

and this concludes the proof of the theorem.

Remark 5.7 Notice that as $|z|^{2},|z|^{2} \log |z|, \operatorname{Re}\left(\gamma_{0} z\right) \in \operatorname{Ker}(\mathscr{L})$, we have

$$
\mathscr{L} v_{\varepsilon}^{i}=-4 \operatorname{Re}\left(\overline{\gamma_{1}} \frac{\bar{z}}{z}\right)+O(|z|),
$$

which implies that

$$
\begin{aligned}
& \mathscr{L}_{g} u_{\varepsilon}^{i}=e^{-\lambda} \tilde{\mathscr{L}}_{g} v_{\varepsilon}^{i}=-4 \alpha_{j} \operatorname{Re}\left(\gamma_{1} z^{2}\right)+O\left(|z|^{3}\right) \\
& \partial_{\nu}\left(\mathscr{L}_{g} u_{\varepsilon}^{i}\right)=-\frac{8 \alpha_{j}}{|z|} \operatorname{Re}\left(\gamma_{1} z^{2}\right)+O\left(|z|^{2}\right),
\end{aligned}
$$

where we used

$$
\begin{aligned}
\partial_{v} & =\frac{x}{|x|} \cdot \nabla=\frac{(z+\bar{z})}{|z|}\left(\partial_{z}+\partial_{\bar{z}}\right)+\frac{(z-\bar{z})}{2 i} i\left(\partial_{z}-\partial_{\bar{z}}\right)=\frac{1}{|z|}\left(z \partial_{z}+\bar{z} \partial_{\bar{z}}\right) \\
& =\frac{2}{|z|} \operatorname{Re}\left(z \partial_{z}(\cdot)\right) .
\end{aligned}
$$

\subsection{Indicial roots analysis: case of ends of higher multiplicity}

Proposition 5.8 Let $1 \leq i \leq n$ and $1 \leq j \neq i \leq n$, and assume that $\vec{\Phi}$ has an end of multiplicity $m \geq 2$ at $p_{j}$, and define $v_{\varepsilon, \delta}^{i}$ in $U_{j}$ as $u_{\varepsilon, \delta}^{i}=e^{\lambda} v_{\varepsilon, \delta}^{i}$. Then there exists $v_{\varepsilon}^{i} \in C^{\infty}\left(\Sigma \backslash\left(\bar{B}_{\varepsilon}\left(p_{i}\right) \cup\left\{p_{1}, \ldots, p_{n}\right\}\right)\right)$ such that for all compact $K \subset \Sigma_{\varepsilon}^{i}$, we have (up to a subsequence as $\delta \rightarrow 0$ )

$$
v_{\varepsilon, \delta}^{i} \underset{\delta \rightarrow 0}{\longrightarrow} v_{\varepsilon}^{i} \quad \text { in } C^{l}(K) \text { for all } l \in \mathbb{N} .
$$


Furthermore, for all $j \neq i$, we have an expansion in $U_{j}$ as

$$
\begin{aligned}
v_{\varepsilon}^{i}(z)= & |z|^{m+1} \sum_{k=1}^{m} \operatorname{Re}\left(\frac{\gamma_{i, j, k}^{0}}{z^{k}}\right)+|z|^{1-m} \sum_{k=0}^{m} \operatorname{Re}\left(\gamma_{i, j, k}^{1} z^{m+k}\right) \\
& +\gamma_{i, j}^{2}|z|^{m+1}+\gamma_{i, j}^{3}|z|^{m+1} \log |z|+\varphi_{\varepsilon}(z)
\end{aligned}
$$

for some real-analytic function $\varphi_{\varepsilon}$ such that $\varphi_{\varepsilon}(z)=O\left(|z|^{m+2}\right)$. Furthermore, we have an expansion

$$
u_{\varepsilon}^{i}(z)=e^{\lambda} v_{\varepsilon}^{i}=\operatorname{Re}\left(\frac{c_{i, j}}{z^{m}}\right)+\sum_{1-m \leq k+l \leq 0} \operatorname{Re}\left(c_{i, j, k, l} z^{k} \bar{z}^{l}\right)+a_{i, j} \log |z|+\psi_{\varepsilon}(z),
$$

for some $\psi_{\varepsilon} \in C^{\infty}(B(0,1) \backslash\{0\})$ such that for all $l \in \mathbb{N}$

$$
\nabla^{l} \psi_{\varepsilon}=O\left(|z|^{1-l}\right),
$$

and the $c_{i, j, k, l}$ are almost all zero, that is all but finitely many as $j, k \in \mathbb{Z}$ and $1-m \leq$ $j+k \leq 0$.

\section{Proof Step 1: Indicial roots analysis.}

We have the expansion

$$
e^{2 \lambda}=\frac{\alpha_{j}^{2}}{|z|^{2 m+2}}\left(1+2 \operatorname{Re}\left(\alpha_{0} z\right)+O\left(|z|^{2}\right)\right) .
$$

Now, let $v_{\varepsilon}^{i}$ such that $u_{\varepsilon}^{i}=e^{\lambda} v_{\varepsilon}^{i}$. Then we have as

$$
e^{\lambda} \Delta_{g} u_{\varepsilon}^{i}=e^{-\lambda} \Delta_{g_{0}}\left(e^{\lambda} v_{\varepsilon}^{i}\right)=\Delta v_{\varepsilon}^{i}+2 \nabla \lambda \cdot \nabla v_{\varepsilon}^{i}+\left(3 \Delta \lambda+|\nabla \lambda|^{2}\right) v_{\varepsilon}^{i}
$$

Now we have

$$
\lambda=-(m+1) \log |z|+\log \left(\alpha_{j}\right)+\log (1+O(|z|)),
$$

so we have $\Delta \lambda \in L^{\infty}\left(D^{2}\right)$ and

$$
\nabla \lambda=-(m+1) \frac{x}{|x|^{2}}+O(1)|\nabla \lambda|^{2}=\frac{(m+1)^{2}}{|x|^{2}}(1+O(|x|))
$$

so we obtain

$$
e^{\lambda} \Delta_{g} u_{\varepsilon}^{i}=\left(\Delta_{g}-2(m+1)\left(\frac{x}{|x|^{2}}+O(1)\right) \cdot \nabla+\left(\frac{(m+1)^{2}}{|x|^{2}}+O\left(\frac{1}{|x|}\right)\right)\right) v_{\varepsilon}^{i}
$$

As $e^{2 \lambda} K_{g}=O(1)$, we finally get

$$
e^{\lambda} \mathscr{L}_{g} u_{\varepsilon}^{i}=\left(\Delta-2(m+1)\left(\frac{x}{|x|^{2}}+O(1)\right) \cdot \nabla+\left(\frac{(m+1)^{2}}{|x|^{2}}+O\left(\frac{1}{|x|}\right)\right)\right) v_{\varepsilon}^{i} .
$$

Now, denote by $\mathscr{L}_{m}$ the elliptic operator with regular singularities (see [32])

$$
\mathscr{L}_{m}=\Delta-2(m+1) \frac{x}{|x|^{2}} \cdot \nabla+\frac{(m+1)^{2}}{|x|^{2}} .
$$

As

$$
\frac{x}{|x|^{2}}=\nabla \log |x|
$$


and $\log$ is harmonic on $D^{2} \backslash\{0\}$, we have

$$
\mathscr{L}_{m}^{*}=\Delta+2(m+1) \frac{x}{|x|^{2}} \cdot \nabla+\frac{(m+1)^{2}}{|x|^{2}},
$$

where $\mathscr{L}_{m}^{*}$ is the formal adjoint of $\mathscr{L}_{m}$. As the indicial roots of on operator of the form

$$
\Delta+\frac{x+b(x)}{|x|^{2}} \cdot \nabla+\frac{c(x)}{|x|^{2}}
$$

where $b$ and $c$ are $C^{\infty}$ and $b(x)=O\left(|x|^{2}\right)$ only depends on $c(0)$ and is independent of $b$ [32].

Therefore, the indicial roots of $e^{\lambda} \mathscr{L}_{g} e^{\lambda}\left(e^{\lambda} \mathscr{L}_{g}\left(e^{\lambda} \cdot\right)\right)$, giving all possible asymptotic behaviour of a solution of $\mathscr{L}_{g}^{2} u_{\varepsilon}^{i}=0$ in $D^{2} \backslash\{0\}$ are the same of the indicial roots of the operator $\mathscr{L}_{m}^{*} \mathscr{L}_{m}$. Therefore, consider first a solution $v$ of

$$
\mathscr{L}_{m}^{*} \mathscr{L}_{m} v=0 .
$$

First, recall that

$$
\begin{aligned}
& \Delta=\partial_{r}^{2}+\frac{1}{r} \partial_{r}+\frac{\Delta_{S^{1}}}{r^{2}} \\
& \mathscr{L}_{m}=\partial_{r}^{2}-\frac{(2 m+1)}{r} \partial_{r}+\frac{\Delta_{S^{1}}+(m+1)^{2}}{r^{2}} .
\end{aligned}
$$

Therefore, for all $k \in \mathbb{N}$ the projection $\mathscr{L}_{m, k}$ on $\operatorname{Span}\left(e^{i \cdot k}\right)$ of $\mathscr{L}_{m}$ is given by

$$
\mathscr{L}_{m, k}=\partial_{r}^{2}-\frac{(2 m+1)}{r} \partial_{r}+\frac{(m+1)^{2}-k^{2}}{r^{2}} .
$$

We first look for solutions of the form

$$
v(r)=r^{\alpha}
$$

for some $\alpha \in \mathbb{C}$. We have by a direct computation

$$
\begin{aligned}
\mathscr{L}_{m, k} v & =\left(\alpha(\alpha-1)-2(m+1) \alpha+(m+1)^{2}-k^{2}\right) r^{\alpha-2} \\
& =\left(\alpha^{2}-2(m+1) \alpha+(m+1)^{2}-k^{2}\right) r^{\alpha-2} \\
& =(\alpha-(m+1+|k|))(\alpha-(m+1-|k|)) r^{\alpha-2} .
\end{aligned}
$$

Therefore, for all $k \in \mathbb{Z} \backslash\{0\}$, we have two linearly independent solutions

$$
v_{k, 0}(r)=r^{m+1+|k|}, \quad v_{k, 1}(r)=r^{m+1-|k|} .
$$

Now, we compute if $\alpha^{\prime}=\alpha+2$

$$
\begin{aligned}
\mathscr{L}_{m, k}^{*} \mathscr{L}_{m} r^{\alpha^{\prime}}= & \mathscr{L}_{m, k}^{*}\left(\alpha^{\prime}-(m+1+|k|)\left(\alpha^{\prime}-(m+1-|k|)\right) r^{\alpha}\right. \\
= & \left(\alpha(\alpha-1)+(2 m+3) \alpha+(m+1)^{2}-k^{2}\right)\left(\alpha^{\prime}-(m+1+|k|)\right) \\
& \left(\alpha^{\prime}-(m+1-|k|)\right) r^{\alpha} \\
= & \left(\alpha^{2}+2(m+1) \alpha+(m+1)^{2}-k^{2}\right) r^{\alpha} \\
= & (\alpha-(-(m+1)+|k|))(\alpha-((-m+1)-|k|)) \\
& \left(\alpha^{\prime}-(m+1+|k|)\right)\left(\alpha^{\prime}-(m+1-|k|)\right)
\end{aligned}
$$


and we find two independent solution for $k \in \mathbb{Z}$

$$
v_{k, 2}(r)=r^{-m+1+|k|}, \quad v_{k, 3}(r)=r^{-m+1-|k|} .
$$

Now, for $k=0$, we need to find two additional solution and one check immediately that

$$
r^{m+1} \log (r), \quad r^{1-m} \log (r)
$$

are two additional solutions. Furthermore, notice that when $|k|=m$,

$$
\left\{r^{m+1+|k|}, r^{m+1-|k|}, r^{1-m+|k|}, r^{1-m-|k|}\right\}=\left\{r^{1-2 m}, r, r^{2 m+1}\right\}
$$

so we need to find another solution. As $\operatorname{Ker}\left(\mathscr{L}_{m, m}^{*}\right)=\operatorname{Span}\left(r^{-(m+1)+m}, r^{-(m+1)-m}\right)=$ $\operatorname{Span}\left(r^{-1}, r^{-(2 m+1)}\right)$, we compute that

$$
\begin{aligned}
\mathscr{L}_{m, m}(r \log (r)) & =\frac{1}{r}-\frac{(2 m+1)}{r}(\log (r)+1)+\frac{(m+1)^{2}-m^{2}}{r^{2}}(r \log (r)) \\
& =-\frac{2 m}{r} \in \operatorname{Ker}\left(\mathscr{L}_{m, m}^{*}\right) .
\end{aligned}
$$

Therefore, for $|k|=m$, we have the basis of solutions

$$
r^{1-2 m}, r, r^{2 m+1}, r \log (r) .
$$

so we find the additional solution $r \log (r)$ when $|k|=m$. Therefore, we finally get

$$
\begin{aligned}
v_{\varepsilon, \delta}^{i}(r, \theta)= & \sum_{k \in \mathbb{Z}^{*}}\left(\gamma_{k}^{1} r^{m+1+k}+\gamma_{k}^{2} r^{m+1-k}+\gamma_{k}^{3} r^{1-m+k}+\gamma_{k}^{4} r^{1-m-k}\right) e^{i k \theta} \\
& +\left(\gamma e^{i m \theta}+\bar{\gamma} e^{-i m \theta}\right) r \log (r)+\gamma_{0}^{1} r^{1-m}+\gamma_{0}^{2} r^{1-m} \log (r) \\
& +\gamma_{0}^{3} r^{m+1}+\gamma_{0}^{4} r^{m+1} \log (r) .
\end{aligned}
$$

Step 2: Estimate coming from $\mathscr{L}_{m} v_{\varepsilon, \delta}^{i} \in L^{2}$. As

$$
\begin{aligned}
& \int_{B_{1} \backslash \bar{B}_{\delta}(0)}\left(\Delta v_{\varepsilon, \delta}^{i}-2(m+1)\left(\frac{x}{|x|^{2}}+\nabla \zeta_{0}\right) \cdot \nabla v_{\varepsilon, \delta}^{i}+\frac{(m+1)^{2}}{|x|^{2}}\left(1+x \cdot \vec{\zeta}_{1}\right) v_{\varepsilon, \delta}^{i}\right)^{2} d x \\
& \leq \int_{\Sigma_{\varepsilon_{0}, \delta}}\left(\mathscr{L}_{g} u_{\varepsilon, \delta}^{i}\right)^{2} d \operatorname{vol}_{g} \leq C \omega\left(\|v\|_{\mathrm{W}^{2,2}(\Sigma)}\right) \\
& \int_{B_{1} \backslash \bar{B}_{\delta}(0)}\left(\Delta v_{\varepsilon, \delta}^{i}+(m+1)(m-1) \frac{v_{\varepsilon, \delta}^{i}}{|x|^{2}}\right)^{2} d x \\
& \quad+4(m+1)(m-1) \int_{B_{1} \backslash \bar{B}_{\delta}(0)}\left(\frac{x}{|x|^{2}} \cdot \nabla v_{\varepsilon, \delta}^{i}-\frac{v_{\varepsilon, \delta}^{i}}{|x|^{2}}\right)^{2} d x \\
& \quad-\int_{B_{1} \backslash \bar{B}_{\delta}(0)}\left(\nabla \zeta_{2} \cdot \nabla v_{\varepsilon, \delta}^{i}-\frac{x}{|x|^{2}} \cdot \vec{\zeta}_{3} v_{\varepsilon, \delta}^{i}\right)^{2} d x \leq C \omega\left(\|v\|_{\mathrm{W}^{2,2}(\Sigma)}\right) .
\end{aligned}
$$

and $m \geq 2$, we deduce by the same argument as Proposition 5.5 that the following three integrals are bounded uniformly in $\varepsilon$ and $\delta$

$$
\int_{B_{1} \backslash \bar{B}_{\delta}}\left(\mathscr{L}_{m} v_{\varepsilon, \delta}^{i}\right)^{2} d x=\int_{B_{1} \backslash \bar{B}_{\delta}(0)}\left(\Delta v_{\varepsilon, \delta}^{i}-2(m+1) \frac{x}{|x|^{2}} \cdot \nabla v_{\varepsilon, \delta}^{i}+\frac{(m+1)^{2}}{|x|^{2}} v_{\varepsilon, \delta}^{i}\right)^{2} d x
$$




$$
\begin{aligned}
& \int_{B_{1} \backslash \bar{B}_{\delta}(0)}\left(\Delta v_{\varepsilon, \delta}^{i}+(m+1)(m-1) \frac{v_{\varepsilon, \delta}^{i}}{|x|^{2}}\right)^{2} d x \\
& \int_{B_{1} \backslash \bar{B}_{\delta}(0)}\left(\frac{x}{|x|^{2}} \cdot \nabla v_{\varepsilon, \delta}^{i}-\frac{v_{\varepsilon, \delta}^{i}}{|x|^{2}}\right)^{2} d x .
\end{aligned}
$$

Now define

$$
\begin{aligned}
& \tilde{\mathscr{L}}_{m}=\Delta+\frac{(m+1)(m-1)}{|x|^{2}} \\
& \mathscr{D}=\frac{x}{|x|^{2}} \cdot \nabla-\frac{1}{|x|^{2}} .
\end{aligned}
$$

Furthermore, notice that for all $k \in \mathbb{Z}^{*}$, if $P_{k}$ is the projection on $\operatorname{Span}\left(e^{i k} \cdot\right)$, then

$$
\begin{aligned}
\operatorname{Ker}\left(P_{k} \mathscr{L}_{m}\right) & =\operatorname{Span}\left(r^{m+1+k}, r^{m+1-k}\right), \\
\operatorname{Ker}\left(P_{k} \widetilde{\mathscr{L}_{m}}\right) \cap \operatorname{Ker}\left(P_{k} \mathscr{D}\right) & =\operatorname{Span}\left(r^{m-1+k}, r^{m-1-k}\right) .
\end{aligned}
$$

Furthermore, for all $\alpha \in \mathbb{Z}$,

$$
\mathscr{D}\left(r^{\alpha} \log (r)\right)=\frac{1}{r} \partial_{r}\left(r^{\alpha} \log (r)\right)-r^{\alpha-2} \log (r)=(\alpha-1) r^{\alpha-2} \log (r)+r^{\alpha-2},
$$

so we deduce that the coefficients $\gamma_{0}^{1}$ and $\gamma_{0}^{2}$ vanish when $\delta \rightarrow 0$, as $|x|^{(1-m)-2}=$ $|x|^{-(m+1)} \notin L^{2}(B(0,1))$. Furthermore, thanks to (5.46) and the proof of Proposition 5.5, we deduce that whenever a power $\alpha=m+1+k, m+1-k, 1-m+k, 1-m+k$ satisfies

$$
\alpha \leq 0
$$

then the corresponding coefficient $\gamma_{k}^{j}$ vanishes as $\delta \rightarrow 0$. Notice that all powers $r^{m+1+k}$, $r^{m+1-k}, r^{1-m+k}$ and $r^{1-m-k}$ are all distinct, except when $|k|=m$, where the powers become either

$$
r^{2 m+1}, r, r, r^{1-2 m}
$$

or

$$
r, r^{2 m+1}, r^{1-2 m}, r .
$$

Notice also that the coefficient $\gamma$ in (5.44) also vanishes as $\gamma r \log (r) e^{ \pm m \theta} \notin \operatorname{Ker}\left(\mathscr{L}_{m}\right)$ (and using the same argument as in the proof of Theorem 6.6). So we have a remaining coefficient in $\operatorname{Re}\left(\gamma_{0} z\right)$ in the expansion of $v_{\varepsilon, \delta}^{i}$ as $\delta \rightarrow 0$, as $r e^{ \pm i m \theta} \in \operatorname{Ker}\left(\mathscr{L}_{m}\right) \cap \operatorname{Ker}\left(\tilde{\mathscr{L}}_{m}\right) \cap \operatorname{Ker}(\mathscr{D})$. Finally, we deduce that as $\delta \rightarrow 0, v_{\varepsilon, \delta}^{i} \underset{\delta \rightarrow 0}{\longrightarrow} v_{\varepsilon}^{i} \in C_{\mathrm{loc}}^{l}\left(\Sigma_{\varepsilon}^{i}\right)$ for all $l \in \mathbb{N}$ such that

$$
\begin{aligned}
v_{\varepsilon}^{i}= & r^{m+1} \sum_{\substack{k \in \mathbb{Z}^{*} \\
k \geq-m}} r^{k}\left(\gamma_{k}^{1} e^{i k \theta}+\gamma_{-k}^{2} e^{-i k \theta}\right)+r^{1-m} \sum_{k \geq m} r^{k}\left(\gamma_{k}^{3} e^{i k \theta}+\gamma_{-k}^{4} e^{-i k \theta}\right) \\
& +\gamma_{0}^{3} r^{m+1}+\gamma_{0}^{4} r^{m+1} \log (r) \\
= & 2 r^{m+1} \sum_{\substack{k \in \mathbb{Z}^{*} \\
k \geq-m}} \operatorname{Re}\left(\gamma_{k}^{1} z^{k}\right)+2 r^{1-m} \sum_{k \geq m} \operatorname{Re}\left(\gamma_{k}^{3} z^{k}\right)+\gamma_{0}^{3} r^{m+1}+\gamma_{0}^{4} r^{m+1} \log (r),
\end{aligned}
$$


where we used $\gamma_{-k}^{2}=\overline{\gamma_{k}^{1}}$ and $\gamma_{-k}^{4}=\overline{\gamma_{k}^{3}}$. The last expansion of $u_{\varepsilon}^{i}$ follows directly from this estimate using (5.41).

Finally, we obtain in the following theorem the expansion as $\varepsilon \rightarrow 0$ of the previously obtained function $u_{\varepsilon}^{i}$. Notice the shift of notation for $v_{\varepsilon}^{i}$.

Theorem 5.9 Let $u_{\varepsilon}^{i} \in C^{\infty}\left(\Sigma_{\varepsilon}^{i}\right)$ be the function constructed in Proposition 5.8, and $v_{\varepsilon}^{i} \in$ $C^{\infty}\left(\Sigma_{\varepsilon}^{i}\right)$ be the global function such that $u_{\varepsilon}^{i}=|\vec{\Phi}|^{2} v_{\varepsilon}^{i}$. Then there exists $v_{0}^{i} \in W^{2,2}(\Sigma)$ such that up to a subsequence,

$$
v_{\varepsilon}^{i} \underset{\varepsilon \rightarrow 0}{\longrightarrow} v_{0}^{i} \quad \text { in } C^{l}\left(\Sigma \backslash\left\{p_{1}, \ldots, p_{n}\right\}\right) \text { for all } l \in \mathbb{N} .
$$

Furthermore, we have $v_{0}^{i}\left(p_{j}\right)=0$ for $j \neq i, v_{0}^{i}\left(p_{i}\right)=v\left(p_{i}\right)$, and for all $1 \leq j \neq i \leq n$, and if $p_{j}$ has multiplicity $m \geq 1, v_{0}^{i}$ admits the following expansion in $U_{j}$ for some $\gamma_{i, j}^{0}, \gamma_{i, j, k, l} \in$ $\mathbb{C}(k, l \in \mathbb{N})$ and $\gamma_{i, j}^{1} \in \mathbb{R}$

$$
v_{0}^{i}(z)=\operatorname{Re}\left(\gamma_{i, j}^{0} z^{m}\right)+\sum_{m+1 \leq k+l \leq 2 m} \operatorname{Re}\left(\gamma_{i, j, k, l} z^{k} \bar{z}^{l}\right)+\gamma_{i, j}^{1}|z|^{2 m} \log |z|+O\left(|z|^{2 m+1} \log |z|\right) .
$$

Furthermore, if $m=1$, there exists $\gamma_{i, j}^{0}, \gamma_{i, j}^{1} \in \mathbb{C}$ and $\gamma_{i, j}^{2}, \gamma_{i, j}^{3} \in \mathbb{R}$ such that for all $1 \leq j \leq n$,

$$
v_{0}^{i}(z)=v\left(p_{j}\right) \delta_{i, j}+\operatorname{Re}\left(\gamma_{i, j}^{0} z+\gamma_{i, j}^{1} z^{2}\right)+\gamma_{i, j}^{2}|z|^{2}+\gamma_{i, j}^{3}|z|^{2} \log |z|+O\left(|z|^{3}\right) .
$$

In particular, for all $1 \leq i \leq n$, the variation $\vec{v}_{i}=v_{0}^{i} \vec{n}_{\vec{\Psi}}$ is an admissible variation of the branched Willmore surface $\overrightarrow{\vec{\Psi}}: \Sigma \rightarrow \mathbb{R}^{3}$.

Proof The first claim on $v_{\varepsilon}^{i}$ follows directly from the uniform bound (5.11) and a standard diagonal argument. Furthermore, as $v_{\varepsilon}^{i}=v=v\left(p_{i}\right)+O(\varepsilon)$ on $\partial B_{\varepsilon}\left(p_{i}\right)$, we deduce that $v_{0}^{i}\left(p_{i}\right)=v\left(p_{i}\right)$. Finally, the expansion in $U_{j}$ follows from Theorem, as

$$
u_{\varepsilon}^{i}=\operatorname{Re}\left(\frac{c_{i, j}}{z^{m}}\right)+\sum_{1-m \leq k+l \leq 0} \operatorname{Re}\left(c_{i, j, k, l} z^{k} \bar{z}^{l}\right)+a_{i, j} \log |z|+\psi_{\varepsilon}(z)
$$

and as $|\vec{\Phi}|^{2}=\beta_{0}^{2}|z|^{-2 m}\left(1+O(|z|)\right.$ ) (for some $\beta_{0}>0$ ), we find that for some $\gamma_{i, j, \varepsilon}^{0}, \gamma_{i, j, k, l, \varepsilon} \in \mathbb{C}$ and $\gamma_{i, j, \varepsilon}^{1} \in \mathbb{R}$

$$
\begin{aligned}
v_{\varepsilon}^{i}= & \operatorname{Re}\left(\gamma_{i, j, \varepsilon}^{0} z^{m}\right)+\sum_{m+1 \leq j+k \leq 2 m} \operatorname{Re}\left(\gamma_{i, j, k, l, \varepsilon} z^{k} \bar{z}^{l}\right)+\gamma_{i, j, \varepsilon}^{1}|z|^{2 m} \log |z| \\
& +O\left(|z|^{2 m+1} \log |z|\right)
\end{aligned}
$$

so as $\varepsilon \rightarrow 0$, by the strong convergence $\gamma_{i, j, k, l, \varepsilon} \rightarrow \gamma_{i, j, k, l} \in \mathbb{C}$ and we get the expected expansion. Finally, the indicial root analysis shows that

$$
\nabla^{2} v_{0}^{i}=\nabla^{2} \operatorname{Re}\left(\gamma_{i, j}^{0} z^{m}\right)+O\left(|z|^{m-1} \log |z|\right)=O(\log |z|) \in \bigcap_{p<\infty} L^{p}(\Sigma)
$$

and as $v_{0}^{i} \in C^{\infty}\left(\Sigma \backslash\left\{p_{1}, \ldots, p_{n}\right\}\right)$, we deduce that

$$
v \in \bigcap_{p<\infty} W^{2, p}(\Sigma)
$$

and this concludes the proof of the theorem. 
Remark 5.10 We emphasize that the variations $v_{0}^{i} \in W^{2,2}(\Sigma)$ are admissible at a branch point $p \in \Sigma$ of order $\theta_{0} \geq 1$ corresponds to an end $p_{j}$ (for some $1 \leq j \leq n$ ) of multiplicity $m=\theta_{0} \geq 1$, and the previous theorem shows that in $U_{j}$

$$
v_{0}^{i}(z)=v\left(p_{i}\right) \delta_{i, j}+\operatorname{Re}\left(\gamma_{i, j} z^{\theta_{0}}\right)+O\left(|z|^{\theta_{0}+1} \log |z|\right),
$$

so these variations are indeed admissible by the discussion in Sect. 3. For more details on this important technical point, we refer to [26]. Notice that in general, at a branch point of multiplicity $m \geq 2$, we have $\nabla^{m+1} v \in L^{\infty}(B(0,1))$ which implies that $v \in C^{m, 1}(B(0,1))$ while for $m=1, \nabla^{2} v=O(\log |z|)$ so that $v \in \bigcap_{\alpha<1} C^{1, \alpha}(B(0,1))$, but $v \notin C^{1,1}(B(0,1))$ in general.

Definition 5.11 For all admissible variation $v \in W^{2,2}(\Sigma)$ of $\vec{\Psi}$ we denote by $u_{0}^{i}=|\vec{\Phi}|^{2} v_{0}^{i}$, where $v_{0}^{i} \in W^{2,2}(\Sigma)$ is the admissible variation of $\vec{\Psi}$ constructed in Theorem 5.9.

\section{Renormalised energy for minimal surfaces with embedded ends}

\subsection{Explicit computation of the singular energy}

First recall the definition of flux of a complete minimal surface.

Definition 6.1 Let $\Sigma$ be a closed Riemann surface, $p_{1}, \ldots, p_{n} \in \Sigma$ be fixed points and $\vec{\Phi}: \Sigma \rightarrow \mathbb{R}^{d}$ be a complete minimal surface with finite total curvature. For all $1 \leq j \leq n$, we define the flux of $\vec{\Phi}$ at $p_{j}$ by

$$
\operatorname{Flux}\left(\vec{\Phi}, p_{j}\right)=\frac{1}{\pi} \operatorname{Im} \int_{\gamma} \partial \vec{\Phi} \in \mathbb{R}^{d}
$$

where $\gamma \subset \Sigma \backslash\left\{p_{1}, \ldots, p_{n}\right\}$ is a fixed contour around $p_{j}$ that does not enclosed other points $p_{k}$ for some $k \neq j$.

By the Weierstrass parametrisation, we have at an end of multiplicity $m \geq 1$ for some $\vec{A}_{0} \in \mathbb{C}^{d} \backslash\{0\}$ and $\vec{A}_{1}, \ldots, \vec{A}_{m} \in \mathbb{C}^{d}$ and $\vec{\gamma}_{0} \in \mathbb{R}^{d}$

$$
\vec{\Phi}(z)=\sum_{j=0}^{m} \operatorname{Re}\left(\frac{\vec{A}_{j}}{z^{m-j}}\right)+\vec{\gamma}_{0} \log |z|+O(|z|),
$$

and we compute

$$
\int_{S^{1}} \partial \vec{\Phi}=\int_{S^{1}} \frac{\vec{\gamma}_{0}}{2} \frac{d z}{z}=\pi i \vec{\gamma}_{0}
$$

Therefore, we have

$$
\operatorname{Flux}\left(\vec{\Phi}, p_{j}\right)=\vec{\gamma}_{0} \in \mathbb{R}^{d}
$$

is a well-defined quantity independent of the chart.

Theorem 6.2 Let $\Sigma$ a compact Riemann surface, $\vec{\Phi}: \Sigma \backslash\left\{p_{1}, \ldots, p_{n}\right\} \rightarrow \mathbb{R}^{3}$ a minimal surface with $n$ embedded ends $p_{1}, \ldots, p_{n} \in \Sigma$ and exactly $m$ catenoid ends $p_{1}, \ldots, p_{m} \in \Sigma$ $(0 \leq m \leq n)$. Let $\vec{\Psi}: \Sigma \rightarrow \mathbb{R}^{3}$ be the inversion at 0 of $\vec{\Phi}$. Then the index quadratic form 
$Q_{\vec{\Psi}}: W^{2,2}\left(\Sigma, \mathbb{R}^{3}\right) \rightarrow \mathbb{R}$ of $\vec{\Psi}$ satisfies for all $\vec{v} \in \operatorname{Var}(\vec{\Psi})$ such that $v=\left\langle\vec{v}, \vec{n}_{\vec{\Psi}}\right\rangle \in C^{2}(\Sigma)$ the identity

$$
\begin{aligned}
Q_{\vec{\Psi}}(\vec{v})=\lim _{\varepsilon \rightarrow 0} & \left\{\frac{1}{2} \int_{\Sigma_{\varepsilon}^{2}}\left(\Delta_{g} u-2 K_{g} u\right)^{2} d \operatorname{vol}_{g}\right. \\
& -8 \pi \sum_{i=1}^{n} \frac{\alpha_{i}^{2}}{\varepsilon^{2}} v^{2}\left(p_{i}\right)-24 \pi \sum_{j=1}^{m} \beta_{j}^{2} \log \left(\frac{1}{\varepsilon}\right) v^{2}\left(p_{j}\right) \\
& \left.+16 \pi \sum_{j=1}^{m} \beta_{j}^{2} v^{2}\left(p_{j}\right)\right\},
\end{aligned}
$$

where $u=|\vec{\Phi}|^{2} v$, and $\Sigma_{\varepsilon}=\Sigma \backslash \bigcup_{i=1}^{n} \bar{B}_{\varepsilon}\left(p_{j}\right)$, where the $\bar{B}_{\varepsilon}\left(p_{i}\right)$ are chosen as in [28] (with respect to a fixed covering $U_{1}, \ldots, U_{n}$ of $p_{1}, \ldots, p_{n}$, see also Proposition ), and

$$
\beta_{j}=\left|\operatorname{Flux}\left(\vec{\Phi}, p_{j}\right)\right| \text {. }
$$

Proof Write the decomposition $\vec{v}=-v \vec{n}_{\vec{\Psi}}+2 \operatorname{Re}(\alpha \otimes \partial \vec{\Psi})$. Since $Q_{\vec{\Psi}}(\vec{v})=Q_{\vec{\Psi}}(-\vec{v})$, we will compute $Q_{\vec{\Psi}}(\vec{v})$ in this proof.

Rather than using Theorem 4.1, we will directly get the minimal condition on $\alpha$ so that $\vec{v}$ becomes admissible.

Let $p_{i}$ be a catenoid end. Then up to a rotation, we may assume that the stereographic projection of the Gauss map $g: \Sigma \rightarrow S^{2}=\mathbb{C} \cup\{\infty\}$ vanishes at $p_{i}\left(g\left(p_{i}\right)=0\right)$. Taking a chart centred at $z=0 \in \mathbb{C}$, this implies that the Weierstrass data can be written as

$$
g(z)=-\lambda_{0} z+\lambda_{1} z^{2}+O\left(|z|^{3}\right), \quad \omega=\left(-\frac{1}{z^{2}}+\frac{\omega_{1}}{z}+\omega_{0}\right) d z+O(|z|) .
$$

Then we have

$$
\left(1-g^{2}, i\left(1+g^{2}\right), 2 g\right) \omega=\left(-\frac{1}{z^{2}}+\frac{\omega_{1}}{z}, i\left(-\frac{1}{z^{2}}+\frac{\omega_{1}}{z}\right), \frac{2 \lambda_{0}}{z}\right) d z+O(1) .
$$

Since $\vec{\Phi}$ is not multi-valued, we deduce that we have

$$
\begin{aligned}
0 & =\operatorname{Re} \int_{S^{1}}\left(1-g^{2}, i\left(1+g^{2}\right), 2 g\right)=\operatorname{Re} \int_{S^{1}}\left(\frac{\omega_{1}}{z}, \frac{i \omega_{1}}{z}, \frac{2 \lambda_{0}}{z}\right) d z \\
& =\operatorname{Re}\left(2 \pi i\left(\omega_{1}, i \omega_{1}, 2 \lambda_{0}\right)\right) \\
& =-2 \pi\left(\operatorname{Im}\left(\omega_{1}\right), \operatorname{Re}\left(\omega_{1}\right), 2 \operatorname{Im}\left(\lambda_{0}\right)\right)
\end{aligned}
$$

so that $\omega_{1}=0$, and $\lambda_{0} \in \mathbb{R}$. Since $p_{i}$ is a catenoid end, we furthermore have $\lambda_{0} \in \mathbb{R} \backslash\{0\}$. Then we get

$$
\left(1-g^{2}, i\left(1+g^{2}\right), 2 g\right) \omega=\left(-\frac{1}{z^{2}}+\lambda_{0}^{2}+\omega_{0}, i\left(-\frac{1}{z^{2}}-\lambda_{0}^{2}+\omega_{0}\right), \frac{2 \lambda_{0}}{z}-2 \lambda_{1}\right)+O(|z|)
$$

Integrating (6.3), we deduce that

$$
\begin{aligned}
\vec{\Phi}(z) & =\operatorname{Re}\left(\int_{*}^{z}\left(1-g^{2}, i\left(1+g^{2}\right), 2 g\right) \omega\right) \\
& =\operatorname{Re}\left(\frac{1}{z}+\left(\lambda_{0}^{2}+\omega_{0}\right), i\left(\frac{1}{z}+\left(-\lambda_{0}^{2}+\omega_{0}\right) z\right), 2 \lambda_{0} \log |z|-2 \lambda_{1} z\right)+O\left(|z|^{2}\right)
\end{aligned}
$$




$$
=\operatorname{Re}\left(\overrightarrow{\vec{A}_{0}}\left(\frac{1}{z}+\omega_{0} z\right)+\lambda_{0}^{2} \vec{A}_{0} z\right)+\vec{e}_{3}\left(2 \lambda_{0} \log |z|-2 \operatorname{Re}\left(\lambda_{1} z\right)\right)+O\left(|z|^{2}\right)
$$

where

$$
\left\{\begin{array}{c}
\vec{A}_{0}=(1,-i, 0) \\
\vec{e}_{3}=(0,0,1)
\end{array}\right.
$$

This allows us to rewrite

$$
\vec{\Phi}(z)=\left(1+\lambda_{0}^{2}|z|^{2}\right) \operatorname{Re}\left(\overrightarrow{\vec{A}}_{0}\left(\frac{1}{z}+\omega_{0} z\right)\right)+\vec{e}_{3}\left(2 \lambda_{0} \log |z|-2 \operatorname{Re}\left(\lambda_{1} z\right)\right)+O\left(|z|^{2}\right) .
$$

Notice that the signed flux of $\vec{\Phi}$ at $p_{i}$ is given for $\delta>0$ small enough by

$$
\left(0,0, \beta_{i}\right)=\frac{1}{\pi} \operatorname{Im} \int_{B_{\delta}\left(p_{i}\right)} \partial \vec{\Phi}=\frac{1}{2 \pi} \operatorname{Im} \int_{B_{\delta}\left(p_{i}\right)}\left(1-g^{2}, i\left(1+g^{2}\right), 2 g\right) \omega=\left(0,0,2 \lambda_{0}\right),
$$

and $\beta_{i}=\left\langle\operatorname{Flux}_{p_{i}}(\vec{\Phi}), \vec{n}_{\vec{\Phi}}\left(p_{i}\right)\right\rangle$. In particular, we deduce since $\left\langle\vec{A}_{0}, \vec{A}_{0}\right\rangle=\left\langle\vec{A}_{0}, \vec{e}_{3}\right\rangle=$ $\left\langle\overline{\vec{A}_{0}}, \vec{e}_{3}\right\rangle=0$ that

$$
\begin{aligned}
|\vec{\Phi}|^{2} & =\left(1+\lambda_{0}^{2}|z|^{2}\right)^{2}\left|\frac{1}{z}+\omega_{0} z\right|^{2}+4 \lambda_{0}^{2} \log ^{2}|z|+O(|z| \log |z|) \\
& =\frac{1}{|z|^{2}}\left(1+4 \lambda_{0}^{2}|z|^{2} \log ^{2}|z|+2 \lambda_{0}^{2}|z|^{2}+2 \operatorname{Re}\left(\omega_{0} z^{2}\right)+O\left(|z|^{3} \log |z|\right)\right) .
\end{aligned}
$$

Therefore, we deduce that

$$
\begin{aligned}
\vec{\Psi}(z)= & \frac{\vec{\Phi}(z)}{|\vec{\Phi}(z)|^{2}}=\left(1-4 \lambda_{0}^{2}|z|^{2} \log ^{2}|z|-2 \lambda_{0}^{2}|z|^{2}-2 \operatorname{Re}\left(\omega_{0} z^{2}\right)+O\left(|z|^{3} \log |z|\right)\right) \\
& \times\left(\left(1+\lambda_{0}^{2}|z|^{2}\right) \operatorname{Re}\left(\overrightarrow{\vec{A}}_{0}\left(\bar{z}+\omega_{0} z^{2} \bar{z}\right)\right)+\vec{e}_{3}\left(2 \lambda_{0}|z|^{2} \log |z|-2 \operatorname{Re}\left(\lambda_{1} z^{2} \bar{z}\right)\right)+O\left(|z|^{3}\right)\right) \\
= & \operatorname{Re}\left(\vec{A}_{0}\left(z-\omega_{0} z^{3}-\lambda_{0}^{2} z^{2} \bar{z}-4 \lambda_{0}^{2} z^{2} \bar{z} \log ^{2}|z|\right)\right) \\
& +\vec{e}_{3}\left(2 \lambda_{0}|z|^{2} \log |z|-2 \operatorname{Re}\left(\lambda_{1} z^{2} \bar{z}\right)\right)+O\left(|z|^{4} \log ^{2}|z|\right) .
\end{aligned}
$$

Now, we compute

$$
\begin{aligned}
\vec{n}_{\vec{\Phi}} & =\left(\frac{2 \operatorname{Re}(g)}{1+|g|^{2}}, \frac{2 \operatorname{Im}(g)}{1+|g|^{2}},-1+\frac{2|g(z)|^{2}}{1+|g(z)|^{2}}\right) \\
& =2 \operatorname{Re}\left(\vec{A}_{0}\left(-\lambda_{0} z+\lambda_{1} z^{2}\right)\right)+\vec{e}_{3}\left(-1+2 \lambda_{0}^{2}|z|^{2}\right) .
\end{aligned}
$$

Therefore, we have

$$
\begin{aligned}
\left\langle\vec{n}_{\vec{\Phi}}, \vec{\Phi}\right\rangle= & \left(1+\lambda_{0}^{2}|z|^{2}\right) \operatorname{Re}\left(\left|\vec{A}_{0}\right|^{2}\left(\frac{1}{z}+\omega_{0} z\right)\left(-\lambda_{0} z+\lambda_{1} z^{2}\right)\right)-2 \lambda_{0} \log |z|+2 \operatorname{Re}\left(\lambda_{1} z\right) \\
& +2 \lambda_{0}^{3}|z|^{2} \log |z|+O\left(|z|^{3}\right) \\
= & -2 \lambda_{0}(\log |z|+1)+4 \operatorname{Re}\left(\lambda_{1} z\right)-2 \lambda_{0}^{3}|z|^{2}-2 \lambda_{0} \operatorname{Re}\left(\omega_{0} z^{2}\right) \\
& +2 \lambda_{0}^{3}|z|^{2} \log |z|+O\left(|z|^{3}\right) .
\end{aligned}
$$

This implies that

$$
2\left\langle\vec{n}_{\vec{\Phi}}, \vec{\Phi}\right\rangle \vec{\Psi}=\left(-4 \lambda_{0}(\log |z|+1)+8 \operatorname{Re}\left(\lambda_{1} z\right)+O\left(|z|^{2}\right)\right)\left(\operatorname{Re}\left(\vec{A}_{0} z\right)\right.
$$




$$
\begin{aligned}
& \left.+\vec{e}_{3}\left(2 \lambda_{0}|z|^{2} \log |z|\right)+O\left(|z|^{3}\right)\right) \\
= & \operatorname{Re}\left(\vec{A}_{0}\left(-4 \lambda_{0} z \log |z|-4 \lambda_{0} z+4 \lambda_{1} z^{2}+4 \overline{\lambda_{1}}|z|^{2}\right)\right) \\
+ & \vec{e}_{3}\left(-8 \lambda_{0}^{2}|z|^{2} \log ^{2}|z|-8 \lambda_{0}^{2}|z|^{2} \log |z|\right)+O\left(|z|^{3}\right) .
\end{aligned}
$$

Finally, we have by Lemma 10.7 the identity $\vec{n}_{\vec{\Psi}}=-\vec{n}_{\vec{\Phi}}+2\left\langle\vec{n}_{\vec{\Phi}}, \vec{\Phi}\right\rangle \frac{\vec{\Phi}}{|\vec{\Phi}|^{2}}$ which implies that

$$
\begin{aligned}
\vec{n}_{\vec{\Psi}}= & \operatorname{Re}\left(\vec{A}_{0}\left(-4 \lambda_{0} z \log |z|-2 \lambda_{0} z+2 \lambda_{1} z^{2}+4 \overline{\lambda_{1}}|z|^{2}\right)\right) \\
& +\vec{e}_{3}\left(1-2 \lambda_{0}^{2}|z|^{2}-8 \lambda_{0}^{2}|z|^{2} \log |z|(\log |z|+1)\right)+O\left(|z|^{3}\right) .
\end{aligned}
$$

Since the end $p_{i}$ is embedded, we deduce that given $v \in W^{2,2}(\Sigma)$ the variation $\vec{v}=-v \vec{n}_{\vec{\Psi}}+$ $2 \operatorname{Re}(\alpha \otimes \partial \vec{\Psi})$ is admissible at $p_{i}$ if and only if $\vec{v} \in W^{2,2} \cap W^{1, \infty}\left(\Sigma, \mathbb{R}^{3}\right)$. Now, by the Sobolev embedding $W^{2,2}(\Sigma) \hookrightarrow C^{0, \alpha}(\Sigma)$ for all $\alpha<1$, we deduce that for all $0<\varepsilon<1$, we have an expansion

$$
v=v\left(p_{i}\right)+O\left(|z|^{1-\varepsilon}\right) .
$$

Therefore, we have

$$
\begin{aligned}
\vec{v}= & \operatorname{Re}\left(\vec{A}_{0}\left(4 \lambda_{0} v\left(p_{i}\right) z \log |z|+2 \lambda_{0} v\left(p_{i}\right) z\right)\right)+2 \operatorname{Re}\left(\alpha\left(\frac{1}{2} \vec{A}_{0}+O(|z|)\right)\right) \\
& +2 \operatorname{Re}\left(2 \lambda_{0} \bar{z} \log |z| \alpha+\lambda_{0} \bar{z} \alpha\right) \vec{e}_{3}+O\left(|z|^{2-\varepsilon}\right) \\
= & \operatorname{Re}\left(\vec{A}_{0}\left(4 \lambda_{0} v\left(p_{i}\right) z \log |z|+\alpha+2 \lambda_{0} v\left(p_{i}\right) z\right)\right) \\
& +2 \operatorname{Re}\left(2 \lambda_{0} \bar{z} \log |z| \alpha+\lambda_{0} \bar{z} \alpha\right) \vec{e}_{3}+O\left(|z|^{2-\varepsilon}\right) .
\end{aligned}
$$

Therefore, $\vec{v}$ is admissible if and only if

$$
\alpha=-4 \lambda_{0} v\left(p_{i}\right) z \log |z|+\beta
$$

where $\beta \in W^{2,2}(\Sigma)$ and $\bar{z} \log |z| \beta \in W^{2,2}(\Sigma)$. In particular, this implies that $\beta\left(p_{i}\right)=0$. However, in the special case of the catenoid ends, we will see that the second variation is independent of $\alpha$ having this precise form.

We will now compute thanks to Theorem $3.3 Q_{\vec{\Psi}}(\vec{v})$, where $\vec{v}=-v \vec{n}_{\vec{\Psi}}+2 \operatorname{Re}(\alpha \otimes \partial \vec{\Psi})$, $v \in C^{2}(\Sigma)$, and where we assume that for some $\gamma_{0} \in \mathbb{R}$, we have

$$
\alpha=\gamma_{0} z \log |z| \frac{1}{d z}+O\left(|z|^{2}\right) .
$$

We also define 1-forms $\omega_{0}(u, \alpha)$ and $\omega_{1}(u, \alpha)$ such that $\omega(u, \alpha)=\omega_{0}(u, \alpha)+\omega_{1}(u, \alpha)$, where

$$
\begin{aligned}
\omega_{0}(u, \alpha)= & \left(\Delta_{g} u+2 K_{g} u+4 \operatorname{Re}\left(g^{-1} \otimes h_{0} \otimes \bar{\partial} \alpha\right)\right)\left(2 \partial u+h_{0} \otimes \alpha\right) \\
- & \partial\left|2 \partial u+h_{0} \otimes \alpha\right|_{g}^{2} \\
& +2 g^{-1} \otimes \bar{\partial}(g \otimes \bar{\alpha}) \otimes\left(2 g^{-1} \otimes h_{0} \otimes \bar{\partial} u-K_{g} g \otimes \bar{\alpha}\right) \\
\omega_{1}(u, \alpha)= & 4\left\langle\vec{\Phi}, \vec{n}_{\vec{\Phi}}\right\rangle g^{-1} \otimes h_{0} \otimes \bar{\partial}\left(|\vec{\Phi}|^{2} v^{2}+\frac{1}{|\vec{\Phi}|^{2}} g \otimes|\alpha|^{2}\right) \\
& -8 g^{-1} \otimes h_{0} \otimes \bar{\partial}\left(|\vec{\Phi}|^{2}\left\langle\vec{\Phi}, \vec{n}_{\vec{\Phi}}\right\rangle v^{2}+2 \operatorname{Re}(\alpha \otimes \log |\vec{\Phi}|)|\vec{\Phi}|^{2} v\right)
\end{aligned}
$$




$$
+4 K_{g}\left(\left\langle\vec{\Phi}, \vec{n}_{\vec{\Phi}}\right\rangle v+2 \operatorname{Re}(\alpha \otimes \partial \log |\vec{\Phi}|)\right) g \otimes \bar{\alpha}
$$

We have by Lemma 10.6

$$
h_{0}=-2 \partial g \otimes \omega=-\frac{2 \lambda \lambda_{0}}{z^{2}} d z^{2}+O\left(\frac{1}{|z|}\right)=-\beta_{i} \frac{d z^{2}}{z^{2}}+O\left(\frac{1}{z}\right) .
$$

Therefore, we have

$$
\alpha \otimes h_{0}=-\gamma_{0} \beta_{i} \frac{\log |z|}{z} d z+O(\log |z|) .
$$

We have by (6.4)

$$
\begin{aligned}
e^{2 \lambda} & =\partial \overline{\bar{\partial}}|\vec{\Phi}|^{2}=\frac{1}{|z|^{4}}+\frac{\beta_{i}^{2}}{2|z|^{2}}-2 \operatorname{Re}\left(\frac{\omega_{0}}{\bar{z}^{2}}\right)+O\left(\frac{1}{|z|}\right) \\
& =\frac{1}{|z|^{4}}\left(1+\frac{\beta_{i}^{2}}{2}|z|^{2}-2 \operatorname{Re}\left(\omega_{0} z^{2}\right)+O\left(|z|^{3}\right)\right) .
\end{aligned}
$$

Next we compute

$$
\begin{gathered}
\bar{\partial} \alpha=\frac{\gamma_{0}}{2} \frac{z}{\bar{z}} \frac{d \bar{z}}{d z}+O(|z|) \\
g^{-1} \otimes h_{0} \otimes \bar{\partial} \alpha=|z|^{4}\left(-\frac{\beta_{i}}{z^{2}}\right) \frac{\gamma_{0}}{2} \frac{z}{\bar{z}}+O\left(|z|^{3}\right)=-\frac{\gamma_{0} \beta_{i}}{2}|z|^{2}+O\left(|z|^{3}\right) .
\end{gathered}
$$

Noticing that for some $\gamma_{1}, \gamma_{2} \in \mathbb{R}$ and $\omega_{1}, \omega_{2}, \omega_{3}, \omega_{4} \in \mathbb{C}$, we have

$$
\begin{aligned}
u & =|\vec{\Phi}|^{2} v=|\vec{\Phi}|^{2} v\left(p_{i}\right)+2 \operatorname{Re}\left(\frac{\omega_{3}}{z}+\omega_{4} \frac{z}{\bar{z}}\right)+\gamma_{2}+O(|z|) \\
& =\left(\frac{1}{|z|^{2}}+\beta_{i}^{2} v^{2}\left(p_{i}\right) \log ^{2}|z|\right) v\left(p_{i}\right)+2 \operatorname{Re}\left(\omega_{1} \frac{z}{\bar{z}}\right)+2 \operatorname{Re}\left(\frac{\omega_{2}}{z}\right)+\gamma_{1}+O(|z|) \\
-K_{g} & =\left|h_{0}\right|_{W P}^{2}=\beta_{i}^{2}|z|^{4}+O\left(|z|^{5}\right),
\end{aligned}
$$

we deduce that

$$
\begin{aligned}
& \Delta_{g} u= 4 v\left(p_{i}\right)-8 \operatorname{Re}\left(\omega_{4} z^{2}\right)+O\left(|z|^{3}\right) \\
& \Delta_{g} u+2 K_{g} u=4 v\left(p_{i}\right)-2 \beta_{i}^{2} v\left(p_{i}\right)|z|^{2}-8 \operatorname{Re}\left(\omega_{4} z^{2}\right)+O\left(|z|^{3}\right), \\
& \Delta_{g} u+2 K_{g} u+4 \operatorname{Re}\left(g^{-1} \otimes h_{0} \otimes \bar{\partial} \alpha\right)=4 v\left(p_{i}\right)-2\left(\beta_{i}^{2} v\left(p_{i}\right)+\gamma_{0} \beta_{i}\right)|z|^{2} \\
&-8 \operatorname{Re}\left(\omega_{4} z^{2}\right)+O\left(|z|^{3}\right) \\
& \partial u=\left(-\frac{v\left(p_{i}\right)}{|z|^{2}}+\beta_{i}^{2} v\left(p_{i}\right) \log |z|-\frac{\omega_{2}}{z}+2 i \operatorname{Im}\left(\omega_{1} \frac{z}{\bar{z}}\right)+O(|z|)\right) \frac{d z}{z} \\
& 2 \partial u+\alpha \otimes h_{0}=\left(-\frac{2 v\left(p_{i}\right)}{|z|^{2}}+\left(2 \beta_{i}^{2} v\left(p_{i}\right)-\gamma_{0} \beta_{i}\right) \log |z|\right.\left.-\frac{2 \omega_{2}}{z}+4 i \operatorname{Im}\left(\omega_{1} \frac{z}{\bar{z}}\right)+O(|z|)\right) \frac{d z}{z} \\
&\left(\Delta_{g} u+2 K_{g} u+4 \operatorname{Re}\left(g^{-1} \otimes h_{0} \otimes \bar{\partial} \alpha\right)\right)\left(2 \partial u+\alpha \otimes h_{0}\right) \\
&=\left(-\frac{8 v^{2}\left(p_{i}\right)}{|z|^{2}}+4 v\left(p_{i}\right)\left(2 \beta_{i}^{2} v\left(p_{i}\right)-\gamma_{0} \beta_{i}\right) v\left(p_{i}\right) \log |z|+4\left(\beta_{i}^{2} v\left(p_{i}\right)+\gamma_{0} \beta_{i}\right) v\left(p_{i}\right)\right.
\end{aligned}
$$




$$
\left.-\frac{8 \omega_{2}}{z} v\left(p_{i}\right)+16 i v\left(p_{i}\right) \operatorname{Im}\left(\omega_{1} \frac{z}{\bar{z}}\right)+16 v\left(p_{i}\right) \operatorname{Re}\left(\omega_{4} \frac{z}{\bar{z}}\right)\right) \frac{d z}{z}+O(1)
$$

$\left|2 \partial u+\alpha \otimes h_{0}\right|^{2}=\left(\frac{4 v^{2}\left(p_{i}\right)}{|z|^{2}}-4\left(2 \beta_{i}^{2} v\left(p_{i}\right)-\gamma_{0} \beta_{i}\right) v\left(p_{i}\right) \log |z|\right.$

$\left.+8 v\left(p_{i}\right) \operatorname{Re}\left(\frac{\omega_{2}}{z}\right)+4\left|\omega_{2}\right|^{2}\right) \frac{|d z|^{2}}{|z|^{4}}$

$\left|2 \partial u+\alpha \otimes h_{0}\right|_{g}^{2}=\left(1-\frac{\beta_{i}^{2}}{2}|z|^{2}+2 \operatorname{Re}\left(\omega_{0} z^{2}\right)+O\left(|z|^{3}\right)\right)\left|2 \partial u+h_{0} \otimes \alpha\right|^{2}$

$$
\begin{aligned}
= & \frac{4 v^{2}\left(p_{i}\right)}{|z|^{2}}-4\left(2 \beta_{i}^{2} v\left(p_{i}\right)-\gamma_{0} \beta_{i}\right) v\left(p_{i}\right) \log |z|-2 \beta_{i}^{2} v^{2}\left(p_{i}\right)+8 v\left(p_{i}\right) \operatorname{Re}\left(\frac{\omega_{2}}{z}\right)+4\left|\omega_{2}\right|^{2} \\
& +8 v^{2}\left(p_{i}\right) \operatorname{Re}\left(\omega_{0} \frac{z}{\bar{z}}\right)+O(|z|)
\end{aligned}
$$

$\partial\left|2 \partial u+\alpha \otimes h_{0}\right|_{g}^{2}=\left(-\frac{4 v^{2}\left(p_{i}\right)}{|z|^{2}}-2\left(2 \beta_{i}^{2} v\left(p_{i}\right)-\gamma_{0} \beta_{i}\right) v\left(p_{i}\right)\right.$

$$
\left.-4 v\left(p_{i}\right) \frac{\omega_{2}}{z}+8 i v^{2}\left(p_{i}\right) \operatorname{Im}\left(\omega_{0} \frac{z}{\bar{z}}\right)+O(|z|)\right) \frac{d z}{z}
$$

$g^{-1} \otimes \bar{\partial}(g \otimes \bar{\alpha})=|z|^{4}\left(1+O\left(|z|^{2}\right)\right) \bar{\partial}\left(\frac{1}{z^{2} \bar{z}} \gamma_{0} \log |z|+O\left(\frac{1}{|z|^{2}}\right)\right)$

$$
=-\gamma_{0} \log |z|+\frac{\gamma_{0}}{2}+O(|z|)
$$

$-K_{g}(g \otimes \bar{\alpha})=\beta_{i}^{2}|z|^{4}(1+O(|z|)) \times|z|^{-4}\left(1+O\left(|z|^{2}\right)\right) \times\left(\gamma_{0} \bar{z} \log |z|+O\left(|z|^{2}\right)\right)$

$=\beta_{i}^{2} \gamma_{0} \bar{z} \log |z|+O\left(|z|^{2}\right)$

$g^{-1} \otimes\left(h_{0} \otimes \bar{\partial} u\right)=|z|^{4}\left(1+O\left(|z|^{2}\right)\right)\left(-\frac{\beta_{i}}{z^{2}}+O\left(\frac{1}{|z|}\right)\right)\left(-\frac{v\left(p_{i}\right)}{\bar{z}|z|^{2}}+O\left(\frac{\log |z|}{|z|}\right)\right) d z$

$$
=\beta_{i} v\left(p_{i}\right) \frac{d z}{z}+O(1)
$$

$2 g^{-1} \otimes \bar{\partial}(g \otimes \bar{\alpha})\left(2 g^{-1} \otimes\left(h_{0} \otimes \bar{\partial} u\right)-K_{g}(g \otimes \bar{\alpha})\right)$

$$
=\left(-4 \gamma_{0} \beta_{i} v\left(p_{i}\right) \log |z|+2 \gamma_{0} \beta_{i} v\left(p_{i}\right)\right) \frac{d z}{z}+O(1) \text {. }
$$

Finally, we deduce by (6.6) that

$$
\begin{aligned}
\omega_{0}(u, \alpha)= & \left(-\frac{8 v^{2}\left(p_{i}\right)}{|z|^{2}}+4 v\left(p_{i}\right)\left(2 \beta_{i}^{2} v\left(p_{i}\right)-\gamma_{0} \beta_{i}\right) v\left(p_{i}\right) \log |z|\right. \\
& +4\left(\beta_{i}^{2} v\left(p_{i}\right)+\gamma_{0} \beta_{i}\right) v\left(p_{i}\right) \\
& \left.-\frac{8 \omega_{2}}{z} v\left(p_{i}\right)+16 i v\left(p_{i}\right) \operatorname{Im}\left(\omega_{1} \frac{z}{\bar{z}}\right)+16 v\left(p_{i}\right) \operatorname{Re}\left(\omega_{4} \frac{z}{\bar{z}}\right)\right) \frac{d z}{z} \\
& +\left(\frac{4 v^{2}\left(p_{i}\right)}{|z|^{2}}+2\left(2 \beta_{i}^{2} v\left(p_{i}\right)-\gamma_{0} \beta_{i}\right) v\left(p_{i}\right)+4 v\left(p_{i}\right) \frac{\omega_{2}}{z}\right. \\
& \left.-8 i v^{2}\left(p_{i}\right) \operatorname{Im}\left(\omega_{0} \frac{z}{\bar{z}}\right)\right) \frac{d z}{z} \\
& +\left(-4 \gamma_{0} \beta_{i} v\left(p_{i}\right) \log |z|+2 \gamma_{0} \beta_{i} v\left(p_{i}\right)\right) \frac{d z}{z}+O(1)
\end{aligned}
$$




$$
\begin{aligned}
= & \left(-\frac{4 v^{2}\left(p_{i}\right)}{|z|^{2}}+8\left(\beta_{i}^{2} v\left(p_{i}\right)-\gamma_{0} \beta_{i}\right) v\left(p_{i}\right) \log |z|+2\left(4 \beta_{i}^{2} v\left(p_{i}\right)+2 \gamma_{0} \beta_{i}\right) v\left(p_{i}\right)\right. \\
& \left.-\frac{4 \omega_{2}}{z} v\left(p_{i}\right)+16 v\left(p_{i}\right) \operatorname{Re}\left(\omega_{4} \frac{z}{\bar{z}}\right)+16 i v\left(p_{i}\right) \operatorname{Im}\left(\omega_{5} \frac{z}{\bar{z}}\right)\right) \frac{d z}{z}+O(1) .
\end{aligned}
$$

for some $\omega_{5}$. Now, we have

$$
\begin{aligned}
|\vec{\Phi}|^{2} v^{2} & =\frac{v^{2}\left(p_{i}\right)}{|z|^{2}}+O\left(\frac{1}{|z|}\right) \\
\frac{1}{|\vec{\Phi}|^{2}} g \otimes|\alpha|^{2} & =\gamma_{0}^{2} \log ^{2}|z|+O(|z| \log |z|) \\
|\vec{\Phi}|^{2} v^{2}+\frac{1}{|\vec{\Phi}|^{2}} g \otimes|\alpha|^{2} & =\frac{v^{2}\left(p_{i}\right)}{|z|^{2}}+O\left(\frac{1}{|z|}\right) \\
\bar{\partial}\left(|\vec{\Phi}|^{2} v^{2}+\frac{1}{|\vec{\Phi}|^{2}} g \otimes|\alpha|^{2}\right) & =-\frac{v^{2}\left(p_{i}\right)}{|z|^{4}} z d \bar{z} \\
g^{-1} \otimes h_{0} \otimes \bar{\partial}\left(|\vec{\Phi}|^{2} v^{2}+\frac{1}{|\vec{\Phi}|^{2}} g \otimes|\alpha|^{2}\right) & =|z|^{4} \times\left(-\frac{\beta_{i}}{z^{2}}\right) \times\left(-\frac{v^{2}\left(p_{i}\right)}{|z|^{4}}\right) z d z \\
& =\beta_{i} v^{2}\left(p_{i}\right) \frac{d z}{z}+O(1) .
\end{aligned}
$$

And finally, by (6.5)

$$
4\left\langle\vec{\Phi}, \vec{n}_{\vec{\Phi}}\right\rangle g^{-1} \otimes h_{0} \otimes \bar{\partial}\left(|\vec{\Phi}|^{2} v^{2}+\frac{1}{|\vec{\Phi}|^{2}} g \otimes|\alpha|^{2}\right)=-4 \beta_{i}^{2} v^{2}\left(p_{i}\right)(\log |z|+1) \frac{d z}{z} .
$$

Next, we have

$$
|\vec{\Phi}|^{2}\left\langle\vec{\Phi}, \vec{n}_{\vec{\Phi}}\right\rangle v^{2}=-\beta_{i}(\log |z|+1) \frac{v^{2}\left(p_{i}\right)}{|z|^{2}}+O\left(\frac{1}{|z|}\right)
$$

and

$$
\begin{aligned}
\log |\vec{\Phi}| & =-\log |z|+\log \left(1+\beta_{i}^{2} \log ^{2}|z|+O\left(|z|^{2}\right)\right) \\
& =-\log |z|+O\left(|z|^{2} \log ^{2}|z|\right)
\end{aligned}
$$

$2 \operatorname{Re}(\alpha \otimes \partial \log |\vec{\Phi}|)=-\gamma_{0} \log |z|+O(|z|)$

$2 \operatorname{Re}(\alpha \otimes \partial \log |\vec{\Phi}|)|\vec{\Phi}|^{2} v=-\gamma_{0} v\left(p_{i}\right) \frac{\log |z|}{|z|}$.

Therefore, we have

$$
\begin{aligned}
& |\vec{\Phi}|^{2}\left\langle\vec{\Phi}, \vec{n}_{\vec{\Phi}}\right\rangle v^{2}+2 \operatorname{Re}(\alpha \otimes \partial \log |\vec{\Phi}|)|\vec{\Phi}|^{2} v \\
& \quad=-\left(\beta_{i} v^{2}\left(p_{i}\right)+\gamma_{0} v\left(p_{i}\right)\right) \frac{\log |z|}{|z|^{2}}-\beta_{i} \frac{v^{2}\left(p_{i}\right)}{|z|^{2}}+O\left(\frac{1}{|z|}\right) .
\end{aligned}
$$

Therefore, we have

$$
\bar{\partial}\left(|\vec{\Phi}|^{2}\left\langle\vec{\Phi}, \vec{n}_{\vec{\Phi}}\right\rangle v^{2}+2 \operatorname{Re}(\alpha \otimes \partial \log |\vec{\Phi}| v)\right)=\left(\beta_{i} v^{2}\left(p_{i}\right)+\gamma_{0} v\left(p_{i}\right)\right) \frac{\log |z|}{|z|^{2}} \frac{d \bar{z}}{\bar{z}}
$$




$$
\begin{aligned}
& -\frac{1}{2}\left(\beta_{i} v^{2}\left(p_{i}\right)+\gamma_{0} v\left(p_{i}\right)\right) \frac{1}{|z|^{2}} \frac{d \bar{z}}{\bar{z}}+\beta_{i} v^{2}\left(p_{i}\right) \frac{1}{|z|^{2}} \frac{d \bar{z}}{\bar{z}} \\
= & \frac{1}{|z|^{2}}\left(\left(\beta_{i} v^{2}\left(p_{i}\right)+\gamma_{0} v\left(p_{i}\right)\right) \log |z|+\frac{1}{2}\left(\beta_{i} v^{2}\left(p_{i}\right)-\gamma_{0} v\left(p_{i}\right)\right)\right) \frac{d \bar{z}}{\bar{z}}
\end{aligned}
$$

and

$$
\begin{aligned}
- & 8 g^{-1} \otimes h_{0} \otimes \bar{\partial}\left(|\vec{\Phi}|^{2}\left\langle\vec{\Phi}, \vec{n}_{\vec{\Phi}}\right\rangle v^{2}+2 \operatorname{Re}(\alpha \otimes \partial \log |\vec{\Phi}| v)\right) \\
= & -8|z|^{4} \times\left(-\frac{\beta_{i}}{z^{2}}\right) \times \frac{1}{|z|^{2}}\left(\left(\beta_{i} v^{2}\left(p_{i}\right)+\gamma_{0} v\left(p_{i}\right)\right) \log |z|\right. \\
& \left.+\frac{1}{2}\left(\beta_{i} v^{2}\left(p_{i}\right)-\gamma_{0} v\left(p_{i}\right)\right)\right) \frac{d z}{\bar{z}}+O(1) \\
= & 8\left(\beta_{i}^{2} v^{2}\left(p_{i}\right)+\beta_{i} \gamma_{0} v\left(p_{i}\right) \log |z|+4\left(\beta_{i}^{2} v^{2}\left(p_{i}\right)-\beta_{i} \gamma_{0} v\left(p_{i}\right)\right)\right) \frac{d z}{z}+O(1)
\end{aligned}
$$

Finally, we have

$$
\begin{aligned}
K_{g} & =O\left(|z|^{4}\right) \\
\left\langle\vec{\Phi}, \vec{n}_{\vec{\Phi}}\right\rangle v & =O(\log |z|) \\
\left\langle\vec{\Phi}, \vec{n}_{\vec{\Phi}}\right\rangle v+2 \operatorname{Re}(\alpha \otimes \partial|\vec{\Phi}|) & =O(\log |z|) \\
g \otimes \bar{\alpha} & =\gamma_{0} \frac{\log |z|}{z|z|^{2}}+O\left(\frac{1}{|z|^{2}}\right) \\
K_{g}\left(\left\langle\vec{\Phi}, \vec{n}_{\vec{\Phi}}\right\rangle v+2 \operatorname{Re}(\alpha \otimes \partial|\vec{\Phi}|)\right) g \otimes \bar{\alpha} & =O\left(|z|^{4}\right) \times O(\log |z|) \times O\left(\frac{\log |z|}{|z|^{3}}\right) \\
& =O\left(|z| \log ^{2}|z|\right) .
\end{aligned}
$$

Finally, we have by (6.7), (6.9), (6.10) and (6.11)

$$
\begin{aligned}
\omega_{1}(u, \alpha)= & 4\left\langle\vec{\Phi}, \vec{n}_{\vec{\Phi}}\right\rangle g^{-1} \otimes h_{0} \otimes \bar{\partial}\left(|\vec{\Phi}|^{2} v^{2}+\frac{1}{|\vec{\Phi}|^{2}} g \otimes|\alpha|^{2}\right) \\
& -g^{-1} \otimes h_{0} \otimes \bar{\partial}\left(|\vec{\Phi}|^{2}\left\langle\vec{\Phi}, \vec{n}_{\vec{\Phi}}\right\rangle v^{2}+2 \operatorname{Re}(\alpha \otimes \log |\vec{\Phi}|) v\right) \\
& +4 K_{g}\left(\left\langle\vec{\Phi}, \vec{n}_{\vec{\Phi}}\right\rangle+2 \operatorname{Re}(\alpha \otimes \partial \log |\vec{\Phi}|)\right) g \otimes \bar{\alpha} \\
= & -4 \beta_{i}^{2} v^{2}\left(p_{i}\right)(\log |z|+1) \frac{d z}{z}+8\left(\beta_{i}^{2} v^{2}\left(p_{i}\right)+\beta_{i} \gamma_{0} v\left(p_{i}\right) \log |z|\right. \\
& \left.+4\left(\beta_{i}^{2} v^{2}\left(p_{i}\right)-\beta_{i} \gamma_{0} v\left(p_{i}\right)\right)\right) \frac{d z}{z}+O(1) \\
= & \left(4\left(\beta_{i}^{2} v^{2}\left(p_{i}\right)+2 \beta_{i} \gamma_{0} v\left(p_{i}\right)\right) \log |z|-4 \beta_{i} \gamma_{0} v\left(p_{i}\right)\right) \frac{d z}{z}+O(1) .
\end{aligned}
$$

Gathering (6.8) and (6.12), we deduce that

$$
\begin{aligned}
\omega(u, \alpha)= & \omega_{0}(u, \alpha)+\omega_{1}(u, \alpha) \\
= & \left(-\frac{4 v^{2}\left(p_{i}\right)}{|z|^{2}}+8\left(\beta_{i}^{2} v\left(p_{i}\right)-\gamma_{0} \beta_{i}\right) v\left(p_{i}\right) \log |z|+2\left(4 \beta_{i}^{2} v\left(p_{i}\right)+2 \gamma_{0} \beta_{i}\right) v\left(p_{i}\right)\right. \\
& \left.-\frac{4 \omega_{2}}{z} v\left(p_{i}\right)+16 v\left(p_{i}\right) \operatorname{Re}\left(\omega_{4} \frac{z}{\bar{z}}\right)+16 i v\left(p_{i}\right) \operatorname{Im}\left(\omega_{5} \frac{z}{\bar{z}}\right)\right) \frac{d z}{z}
\end{aligned}
$$




$$
\begin{aligned}
& +\left(4\left(\beta_{i}^{2} v^{2}\left(p_{i}\right)+2 \beta_{i} \gamma_{0} v\left(p_{i}\right)\right) \log |z|-4 \beta_{i} \gamma_{0} v\left(p_{i}\right)\right) \frac{d z}{z}+O(1) \\
= & \left(-\frac{4 v^{2}\left(p_{i}\right)}{|z|^{2}}+12 \beta_{i}^{2} v^{2}\left(p_{i}\right) \log |z|+8 \beta_{i}^{2} v^{2}\left(p_{i}\right)-\frac{4 \omega_{2}}{z} v\left(p_{i}\right)\right. \\
& \left.+16 v\left(p_{i}\right) \operatorname{Re}\left(\omega_{4} \frac{z}{\bar{z}}\right)+16 i v\left(p_{i}\right) \operatorname{Im}\left(\omega_{5} \frac{z}{\bar{z}}\right)+O(|z|)\right) \frac{d z}{z} .
\end{aligned}
$$

Therefore, we obtain the formula

$$
\operatorname{Im} \int_{\partial B_{\varepsilon}\left(p_{i}\right)} \omega(u, \alpha)=-8 \pi \frac{v^{2}\left(p_{i}\right)}{\varepsilon^{2}}-24 \beta_{i}^{2} v^{2}\left(p_{i}\right) \log \left(\frac{1}{\varepsilon}\right)+16 \pi \beta_{i}^{2} v^{2}\left(p_{i}\right)+O(\varepsilon) .
$$

We finally deduce that

$$
\begin{aligned}
Q_{\vec{\Psi}}(\vec{v})= & \lim _{\varepsilon \rightarrow 0}\left(\frac{1}{2} \int_{\Sigma_{\varepsilon}}\left(\mathscr{L}_{g} u\right)^{2} d \operatorname{vol}_{g}-8 \pi \sum_{i=1}^{n} \frac{\alpha_{i}^{2}}{\varepsilon^{2}} v^{2}\left(p_{i}\right)\right. \\
& \left.-24 \pi \sum_{i=1}^{n} \beta_{i}^{2} \log \left(\frac{1}{\varepsilon}\right)+16 \pi \sum_{i=1}^{n} \beta_{i}^{2} v^{2}\left(p_{i}\right)\right),
\end{aligned}
$$

which is a finite quantity thanks to the previous computations.

Remark6.3 To see that the limit is well-defined, if $v$ is a smooth function, we have the following expansions for some $\gamma_{0} \in \mathbb{C}$

$$
\begin{aligned}
u & =|\vec{\Phi}|^{2} v=|\vec{\Phi}|^{2} v\left(p_{i}\right)+\operatorname{Re}\left(\frac{\gamma_{0}}{z}\right)+O\left(|z| \log ^{2}|z|\right) \\
\Delta_{g} u & =4 v\left(p_{i}\right)+O\left(|z|^{3} \log ^{2}|z|\right) \\
-2 K_{g} u & =2 \beta_{i}^{2}|z|^{2} v\left(p_{i}\right)+O\left(|z|^{3}\right) \\
g & =\frac{1}{|z|^{4}}\left(1+\frac{\beta_{i}^{2}}{2}|z|^{2}+O\left(|z|^{3}\right)\right)|d z|^{2} .
\end{aligned}
$$

Therefore, we deduce that

$$
\begin{aligned}
& \frac{1}{2} \int_{B_{1} \backslash B_{\varepsilon}\left(p_{i}\right)}\left(\Delta_{g} u-2 K_{g} u\right)^{2} d \mathrm{vol}_{g} \\
& \quad=\frac{1}{2} \int_{B_{1} \backslash \bar{B}(0, \varepsilon)}\left(4 v\left(p_{i}\right)+2 \beta_{i}^{2} v\left(p_{i}\right)|z|^{2}+O\left(|z|^{3}\right)\right)^{2}\left(1+\frac{\beta_{i}^{2}}{2}|z|^{2}\right) \frac{|d z|^{2}}{|z|^{4}} \\
& \quad=\frac{1}{2} \int_{B_{1} \backslash \bar{B}(0, \varepsilon)}\left(16 v^{2}\left(p_{i}\right)+16 \beta_{i}^{2} v^{2}\left(p_{i}\right)+O\left(|z|^{3}\right)\right)\left(1+\frac{\beta_{i}^{2}}{2}|z|^{2}+O(|z|)\right) \frac{|d z|^{2}}{|z|^{4}} \\
& \quad=\frac{1}{2} \int_{B_{1} \backslash \bar{B}(0, \varepsilon)}\left(16 v^{2}\left(p_{i}\right)+24 \beta_{i}^{2} v^{2}\left(p_{i}\right)|z|^{2}\right) \frac{|d z|^{2}}{|z|^{4}}+O(1) \\
& \quad=\pi \int_{\varepsilon}^{1}\left(\frac{16 v^{2}\left(p_{i}\right)}{r^{3}}+\frac{24 \beta_{i}^{2} v^{2}\left(p_{i}\right)}{r}\right) d r+O(1) \\
& =\frac{8 \pi}{\varepsilon^{2}}+24 \pi \beta_{i}^{2} v^{2}\left(p_{i}\right) \log \left(\frac{1}{\varepsilon}\right)+O(1)
\end{aligned}
$$

and this proves that (6.1) makes sense. 
Remark 6.4 Now, if we rather choose the variation of Theorem 4.1 given in (4.2), the additional terms do not change in

$$
\int_{\partial B_{\varepsilon}\left(p_{i}\right)} \omega(u, \alpha)
$$

up to a $O(\varepsilon)$ error. Indeed, the additional term in $4 \operatorname{Re}\left(g^{-1} \otimes h_{0} \otimes \bar{\partial} \alpha\right)$ is

$$
4 \operatorname{Re}\left(|z|^{4}\left(-\beta_{i} \frac{1}{z^{2}} \gamma_{2}\right)\right)=-4 \beta_{i} \operatorname{Re}\left(\overline{\gamma_{2}} z^{2}\right)
$$

which will be negligible integrated against $2 \partial u+\alpha \otimes h_{0}$. Then, we have

$$
\begin{aligned}
2 \partial u+h_{0} \otimes \alpha & =\left(-\frac{2 v^{2}\left(p_{i}\right)}{|z|^{2}}+\left(2 \beta_{i}^{2} v\left(p_{i}\right)-\gamma_{0} \beta_{i} v\left(p_{i}\right)\right)\right) \frac{d z}{z}+\left(-\beta_{i} \frac{1}{z^{2}}\right)\left(\gamma_{1} z+\gamma_{2} \bar{z}\right) d z \\
& =\left(-\frac{2 v^{2}\left(p_{i}\right)}{|z|^{2}}+\left(2 \beta_{i}^{2} v\left(p_{i}\right)-\gamma_{0} \beta_{i} v\left(p_{i}\right)\right)-\beta_{i} \gamma_{1}-\beta_{i} \gamma_{2} \frac{\bar{z}}{z}\right) \frac{d z}{z}
\end{aligned}
$$

which shows that the additional non-negligible term in $\left(\Delta_{g} u+2 K_{g} u+4 \operatorname{Re}\left(g^{-1} \otimes \bar{\partial} \alpha\right)\right)(2 \partial u+$ $\left.h_{0} \otimes \alpha\right)$ is

$$
-4 \beta_{i} \gamma_{1} v\left(p_{i}\right) \frac{d z}{z}
$$

We also see easily that there are no additional terms in $\partial\left|2 \partial u+h_{0} \otimes \alpha\right|_{g}^{2}$. Then, the additional term in $g^{-1} \otimes \bar{\partial}(g \otimes \bar{\alpha})$ is

$$
|z|^{4} \partial_{\bar{z}}\left(\frac{\overline{\gamma_{1}}}{z^{2} \bar{z}}+\frac{\overline{\gamma_{2}}}{z \bar{z}^{2}}\right)=-\overline{\gamma_{1}}-2 \overline{\gamma_{2}} \frac{z}{\bar{z}}
$$

which implies that the non-negligible additional term in $2 g^{-1} \otimes \bar{\partial}(g \otimes \bar{\alpha})\left(2 g^{-1} \otimes\left(h_{0} \otimes\right.\right.$ $\left.\bar{\partial} u)-K_{g}(g \otimes \bar{\alpha})\right)$ is

$$
-4 \beta_{i} \overline{\gamma_{1}} v\left(p_{i}\right) \frac{d z}{z}
$$

Summing (6.13) and (6.14), we deduce that the additional term in $\omega_{0}(u, \alpha)$ is

$$
-8 \operatorname{Re}\left(\gamma_{1}\right) \beta_{i} v\left(p_{i}\right) \frac{d z}{z}
$$

As previously the $\alpha$ component in

$$
4\left\langle\vec{\Phi}, \vec{n}_{\vec{\Phi}}\right\rangle g^{-1} \otimes h_{0} \otimes \bar{\partial}\left(|\vec{\Phi}|^{2} v^{2}+\frac{1}{|\vec{\Phi}|^{2}} g \otimes|\alpha|^{2}\right)
$$

is negligible, so the only additional term comes from

$$
-8 g^{-1} \otimes h_{0} \otimes \bar{\partial}\left(|\vec{\Phi}|^{2}\left\langle\vec{\Phi}, \vec{n}_{\vec{\Phi}}\right\rangle v^{2}+2 \operatorname{Re}(\alpha \otimes \log |\vec{\Phi}|)|\vec{\Phi}|^{2} v\right)
$$

and is equal to

$$
\begin{aligned}
& -8|z|^{4}\left(-\frac{\beta_{i}}{z^{2}}\right) \bar{\partial}\left(2 \operatorname{Re}\left(\left(\gamma_{1} z+\gamma_{2} \bar{z}\right)\left(-\frac{1}{2 z}\right)\right) \frac{v\left(p_{i}\right)}{|z|^{2}}\right) \frac{d z}{d \bar{z}} \\
& =8 \beta_{i} \bar{z}^{2} \bar{\partial}\left(-\operatorname{Re}\left(\gamma_{1}\right) \frac{v\left(p_{i}\right)}{|z|^{2}}-\operatorname{Re}\left(\frac{\gamma_{2} v\left(p_{i}\right)}{z^{2}}\right)\right) \frac{d z}{d \bar{z}}
\end{aligned}
$$




$$
=\left(8 \operatorname{Re}\left(\gamma_{1}\right) \beta_{i} v\left(p_{i}\right)+8 \overline{\gamma_{2}} \beta_{i} v\left(p_{i}\right) \frac{z}{\bar{z}}\right) \frac{d z}{z}
$$

which shows by (6.15) that the additional non-negligible term in $\omega(u, \alpha)$ is equal to 0 .

Remark 6.5 For a Willmore surface having its first residue non-vanishing, the Willmore equation is not satisfied every where (see [1]). In the case of inversions of minimal surfaces, for all admissible variation $\vec{v}=v \vec{n}_{\vec{\Phi}}+2 \operatorname{Re}(\alpha \otimes \partial \vec{\Psi})$, we compute as in [28]

$$
\begin{aligned}
D \mathscr{W}(\vec{\Psi})(\vec{v}) & =\frac{d}{d t} \mathscr{W}\left(\vec{\Psi}_{t}\right)_{\mid t=0}=\left.\frac{d}{d t} \mathscr{W}\left(\vec{\Phi}_{t}\right)\right|_{t=0}=D \mathscr{W}(\vec{\Phi})(\vec{u}) \\
& =\int_{\Sigma} d \operatorname{Im}\left(2\langle\vec{H}, \partial \vec{u}\rangle-2 g^{-1} \otimes\left(\vec{h}_{0} \dot{\otimes} \bar{\partial}^{\perp} \vec{u}\right)\right) \\
& =-2 \int_{\Sigma} d \operatorname{Im}\left(g^{-1} \otimes \vec{h}_{0} \dot{\otimes} \bar{\partial}^{\perp} \vec{u}\right) .
\end{aligned}
$$

where $\vec{u}=|\vec{\Phi}|^{2} \vec{v}-2\langle\vec{\Phi}, \vec{v}\rangle \vec{\Phi}=-\left(|\vec{\Phi}|^{2} v\right) \vec{n}_{\vec{\Phi}}+2 \operatorname{Re}(\alpha \otimes \partial \vec{\Phi})$ (we used that $\vec{H}=0$ in the last identity). By Stokes theorem, we deduce that

$$
D \mathscr{W}(\vec{\Psi})(\vec{v})=\lim _{\varepsilon \rightarrow 0} \sum_{i=1}^{n} \operatorname{Im} \int_{\partial B_{\varepsilon}\left(p_{i}\right)} 2 g^{-1} \otimes \vec{h}_{0} \dot{\otimes} \bar{\partial}^{\perp} \vec{u} .
$$

If $(g, \omega)$ are the Weierstrass data of $\vec{\Phi}$, by the previous computations, for all $i=1, \ldots, n$, there exists $\beta_{i} \in \mathbb{R}$ such that $\left|\beta_{i}\right|=\mid$ Flux $_{p_{i}}(\vec{\Phi}) \mid$, and since $h_{0}=-2 \partial g \otimes \omega$ by Lemma 10.6, we find at $p_{i}$ that

$$
h_{0}=-\beta_{i} \frac{d z^{2}}{z^{2}}+O\left(\frac{1}{|z|}\right) .
$$

Notice that this expansion shows that the quantity $-\beta_{i}$ is well-defined independently of the chart as the residue associated to the pole of order 2 of a meromorphic quadratic differential [22]. Up to scaling, we can assume that

$$
|\vec{\Phi}|^{2}=\frac{1}{|z|^{2}}+O\left(\log ^{2}|z|\right)
$$

Finally, without loss of generality, we can assume that the variation is normal (since the non-normal variation is negligible at order 1 by the proof of the previous theorem), and if $\vec{v}=v \vec{n}_{\vec{\Psi}}$, we have $\vec{u}=-|\vec{\Phi}|^{2} v \vec{n}_{\vec{\Phi}}=-u \vec{n}_{\vec{\Phi}}$ and we find

$$
\begin{aligned}
g^{-1} \otimes \vec{h}_{0} \otimes \bar{\partial} \vec{u} & =-g^{-1} \otimes h_{0} \otimes \bar{\partial} u=-|z|^{4}\left(-\beta_{i} \frac{d z^{2}}{z^{2}}\right)\left(\frac{v\left(p_{i}\right)}{|z|^{2} \bar{z}}\right) d z+O(1) \\
& =\beta_{i} v\left(p_{i}\right) \frac{d z}{z}+O(1) .
\end{aligned}
$$

Therefore, we deduce that

$$
\operatorname{Im} \int_{\partial B_{\varepsilon}\left(p_{i}\right)} 2 g^{-1} \otimes \vec{h}_{0} \dot{\otimes} \bar{\partial} \vec{u}=4 \pi \beta_{i} v\left(p_{i}\right)+O(\varepsilon)
$$

and

$$
D \mathscr{W}(\vec{\Psi})(\vec{v})=4 \pi \sum_{i=1}^{n} \beta_{i} v\left(p_{i}\right)
$$


In other words, using the weak formulation of the Willmore equation after Rivière [34], $\vec{\Psi}$ solves in the weak sense the equation

$$
d\left(* d \vec{H}-3 *(d \vec{H})^{\perp}+*(\vec{H} \wedge d \vec{n})\right)=4 \pi \sum_{i=1}^{n} \beta_{i}\left\langle\vec{n}_{\vec{\Psi}}\left(p_{i}\right), \vec{\delta}_{p_{i}}\right\rangle,
$$

where $-\beta_{i}=\operatorname{Res}_{p_{i}}\left(h_{0}\right)$ is the Residue of the meromorphic quadratic differential $h_{0}$ at $p_{i}$, while $\delta_{p_{i}}$ is the Dirac mass and $\vec{\delta}_{p_{i}}=\left(\delta_{p_{i}}, \delta_{p_{i}}, \delta_{p_{i}}\right)$. In particular, we deduce that Willmore surfaces with embedded minimal surfaces with at least one catenoid end are onesided unstable, that is, for all $v \in W^{2,2}(\Sigma)$ such that $\sum_{i=1}^{n} \beta_{i} v\left(p_{i}\right) \neq 0$, if $\vec{v}$ is as previously, we have either $\mathscr{W}\left(\vec{\Psi}_{t}\right)<\mathscr{W}(\vec{\Psi})$ for all $t>0$ small enough or $\mathscr{W}\left(\vec{\Psi}_{t}\right)<\mathscr{W}(\vec{\Psi})$ for all $t<0$ small enough. Furthermore, since the order of branch point is preserved, the integral of the Gauss curvature remains constant and $\vec{\Psi}$ is also one-sided unstable for $W$.

In particular, it will be necessary to restrict to variations such that $\sum_{i=1}^{n} \beta_{i} v\left(p_{i}\right)=0$ in the following so that the definition of index using the second derivative makes any sense. Indeed, we will have

$$
\mathscr{W}\left(\vec{\Psi}_{t}\right)=\mathscr{W}(\vec{\Psi})+4 \pi t \sum_{i=1}^{n} \beta_{i} v\left(p_{i}\right)+\frac{1}{2} t^{2} Q_{\vec{\Psi}}(\vec{v})+o\left(t^{2}\right),
$$

so that the second derivative becomes negligible if the first one does not vanish.

\subsection{Renormalised energy identity}

Theorem 6.6 Under the hypothesis of Theorem A, assume that $\vec{\Phi}$ has embedded ends, let $v \in C^{2}(\Sigma)$, and $\vec{v}=-v \vec{n}_{\vec{\Psi}}+2 \operatorname{Re}(\alpha \otimes \partial \vec{\Psi})$, where $\alpha$ is given by the proof of Theorem 6.2 . There exists a symmetric $\left\{\lambda_{i, j}\right\}_{1 \leq i, j \leq n}$ with zero diagonal terms independent of $v$, and $a$ function $v_{0} \in W^{2,2}(\Sigma)$ vanishing on $\left\{p_{1}, \ldots, p_{n}\right\}$ such that

$$
Q_{\vec{\Psi}}(\vec{v})=\frac{1}{2} \int_{\Sigma}\left(\mathscr{L}_{g} u_{0}\right)^{2} d \operatorname{vol}_{g}+8 \pi \sum_{i=1}^{m} \beta_{i}^{2} v^{2}\left(p_{i}\right)+4 \pi \sum_{1 \leq i, j \leq n} \lambda_{i, j} v\left(p_{i}\right) v\left(p_{j}\right),
$$

where $u_{0}=|\vec{\Phi}|^{2} v_{0}$. In particular, $\operatorname{Ind}_{\mathscr{W}}(\vec{\Psi}) \leq n-1$.

Proof We fix $\varepsilon>0$ small enough such that the ball $\left\{\bar{B}_{2 \varepsilon}\left(p_{i}\right)\right\}_{1 \leq i \leq n}$ are disjoint, and we define the following symmetric bilinear form $B_{\varepsilon}: \mathrm{W}^{2,2}\left(\Sigma_{\varepsilon}\right) \times \mathrm{W}^{\overline{2}, 2}\left(\Sigma_{\varepsilon}\right) \rightarrow \mathbb{R}$

$$
B_{\varepsilon}\left(u_{1}, u_{2}\right)=\int_{\Sigma_{\varepsilon}} \mathscr{L}_{g} u_{1} \mathscr{L}_{g} u_{2} d \operatorname{vol}_{g}
$$

and $Q_{\varepsilon}: \mathrm{W}^{2,2}\left(\Sigma_{\varepsilon}^{2}\right) \rightarrow \mathbb{R}$ the associated quadratic form. We note that

$$
\begin{aligned}
Q(u)= & \lim _{\varepsilon \rightarrow 0} Q_{\varepsilon}(u)-8 \pi \sum_{i=1}^{n} \frac{\alpha_{i}^{2}}{\varepsilon^{2}} v^{2}\left(p_{i}\right)-24 \pi \sum_{j=1}^{m} \beta_{j}^{2} \log \left(\frac{1}{\varepsilon}\right) v^{2}\left(p_{j}\right) \\
& +16 \pi \sum_{j=1}^{m} \beta_{j}^{2} v^{2}\left(p_{j}\right)
\end{aligned}
$$


if $u=|\vec{\Phi}|^{2} v$. We now define $u_{\varepsilon}=u-\sum_{i=1}^{n} u_{\varepsilon}^{i}$

$$
\begin{aligned}
Q_{\varepsilon}(u)= & Q_{\varepsilon}\left(u_{\varepsilon}+\sum_{i=1}^{n} u_{\varepsilon}^{i}\right)=Q_{\varepsilon}\left(u_{\varepsilon}\right)+\sum_{i=1}^{n} Q_{\varepsilon}\left(u_{\varepsilon}^{i}\right) \\
& +\sum_{i=1}^{n} B_{\varepsilon}\left(u_{\varepsilon}, u_{\varepsilon}^{i}\right)+\frac{1}{2} \sum_{1 \leq i \neq j \leq n} B_{\varepsilon}\left(u_{\varepsilon}^{i}, u_{\varepsilon}^{j}\right) .
\end{aligned}
$$

Step 1: Estimation of $Q_{\varepsilon}\left(u_{\varepsilon}\right)$. We first remark that $Q_{\varepsilon}\left(u_{\varepsilon}\right)$ cannot depend on the derivatives of $v$ at $p_{1}, \ldots, p_{n}$ by Sobolev embedding theorem. Therefore, each time we differentiate $v_{\varepsilon}^{i}$, we know that analogous cancellations as observed by the explicit computations in [28] will actually make these residues vanish as $\varepsilon \rightarrow 0$. Whenever one of these terms occur, we shall neglect them.

For all $1 \leq i \leq n$, let $v_{\varepsilon}^{i} \in C^{\infty}\left(\overline{B_{2 \varepsilon} \backslash \bar{B}_{\varepsilon}\left(p_{i}\right)}\right)$ such that $u_{\varepsilon}^{i}=|\vec{\Phi}|^{2} v_{\varepsilon}^{i}$ on $\bar{B}_{2 \varepsilon}\left(p_{i}\right) \backslash B_{\varepsilon}\left(p_{i}\right)$. We fix a chart $D^{2} \rightarrow B_{r}\left(p_{i}\right)$. We recall that close to $p_{i}$, we have

$$
|\vec{\Phi}(x)|^{2}=\frac{\alpha_{i}^{2}}{|x|^{2}}+\beta_{i}^{2} \log ^{2}|x|+O(|x| \log |x|)
$$

Then we deduce by the Dirichlet boundary condition that

$$
v_{\varepsilon}^{i}=v \quad \text { on } \partial B_{\varepsilon}\left(p_{i}\right)
$$

and

$$
\partial_{\nu} u_{\varepsilon}^{i}=\partial_{\nu}|\vec{\Phi}|^{2} v_{\varepsilon}^{i}+|\vec{\Phi}|^{2} \partial_{\nu} v_{\varepsilon}^{i}=\partial_{\nu}|\vec{\Phi}|^{2} v+|\vec{\Phi}|^{2} \partial_{\nu} v_{\varepsilon}^{i} \quad \text { on } \partial B_{\varepsilon}\left(p_{i}\right)
$$

and as

$$
\partial_{\nu} u_{\varepsilon}^{i}=\partial_{\nu}\left(|\vec{\Phi}|^{2}\right) v+|\vec{\Phi}|^{2} \partial_{\nu} v \text { on } \partial B_{\varepsilon}\left(p_{i}\right)
$$

we also have

$$
\partial_{\nu} v_{\varepsilon}^{i}=\partial_{\nu} v \text { on } \partial B_{\varepsilon}\left(p_{i}\right)
$$

so

$$
\begin{aligned}
u_{\varepsilon}^{i} & =\left(\frac{\alpha_{i}^{2}}{\varepsilon^{2}}+\beta_{i}^{2} \log ^{2}(\varepsilon)\right) v+O(1) \\
\partial_{\nu} u_{\varepsilon}^{i} & =\left(-2 \frac{\alpha_{i}^{2}}{\varepsilon^{3}}+2 \frac{\beta_{i}^{2}}{\varepsilon} \log \varepsilon\right) v+\left(\frac{\alpha_{i}^{2}}{\varepsilon^{2}}+\beta_{i}^{2} \log ^{2} \varepsilon\right) \partial_{\nu} v+O(1)
\end{aligned}
$$

then on $B_{2 \varepsilon}\left(p_{i}\right) \backslash \bar{B}_{\varepsilon}\left(p_{i}\right)$, we have

$$
\begin{aligned}
\Delta_{g} u_{\varepsilon}^{i} & =\Delta_{g}\left(|\vec{\Phi}|^{2} v_{\varepsilon}^{i}\right)=4 v_{\varepsilon}^{i}+2\left\langle d|\vec{\Phi}|^{2}, d v_{\varepsilon}^{i}\right\rangle+|\vec{\Phi}|^{2} \Delta_{g} v_{\varepsilon}^{i} \\
& =4 v_{\varepsilon}^{i}-4 \frac{|x|^{4}}{\alpha_{i}^{2}}\left(\frac{\alpha_{i}^{2}}{|x|^{3}}+\beta_{i}^{2} \frac{\log \left(\frac{1}{|x|}\right)}{|x|}\right) \frac{x}{|x|} \cdot \nabla v_{\varepsilon}^{i}+|x|^{2} \Delta v_{\varepsilon}^{i}+O\left(|x|^{4} \log ^{2}|x|\right) \\
& =4 v_{\varepsilon}^{i}-4|x|\left(1+\frac{\beta_{i}^{2}}{\alpha_{i}^{2}}|x|^{2} \log \left(\frac{1}{|x|}\right)\right) \frac{x}{|x|} \cdot \nabla v_{\varepsilon}^{i}+|x|^{2} \Delta v_{\varepsilon}^{i}+O\left(|x|^{4} \log ^{2}|x|\right)
\end{aligned}
$$


and

$$
-2 K_{g} u_{\varepsilon}^{i}=2 \frac{\beta_{i}^{2}}{\alpha_{i}^{2}}|x|^{2} v_{\varepsilon}^{i}+O\left(|x|^{4}\right)
$$

so on $\partial B_{\varepsilon}\left(p_{i}\right)$,

$$
\mathscr{L}_{g} u_{\varepsilon}^{i}=2\left(2+\frac{\beta_{i}^{2}}{\alpha_{i}^{2}} \varepsilon^{2}\right) v-4 \varepsilon\left(1+\frac{\beta_{i}^{2}}{\alpha_{i}^{2}} \varepsilon^{2} \log \left(\frac{1}{\varepsilon}\right)\right) \partial_{\nu} v+\varepsilon^{2} \Delta v_{\varepsilon}^{i}+O\left(\varepsilon^{4} \log ^{2} \varepsilon\right)
$$

Therefore

$$
\begin{aligned}
\frac{x}{|x|} \cdot \nabla\left(\Delta_{g} u_{\varepsilon}^{i}\right)= & 4 \frac{x}{|x|} \cdot \nabla v_{\varepsilon}^{i}-4\left(1+\frac{\beta_{i}^{2}}{\alpha_{i}^{2}}|x|^{2} \log \left(\frac{1}{|x|}\right)\right) \frac{x}{|x|} \cdot \nabla v_{\varepsilon}^{i} \\
& -4 \frac{\beta_{i}^{2}}{\alpha_{i}^{2}}|x|^{2}\left(1+2 \log \left(\frac{1}{|x|}\right)\right) \frac{x}{|x|} \cdot \nabla v_{\varepsilon}^{i} \\
& -4|x|\left(1+\frac{\beta_{i}^{2}}{\alpha_{i}^{2}}|x|^{2} \log \left(\frac{1}{|x|}\right)\right)\left(\frac{x}{|x|}\right)^{t} D^{2} v_{\varepsilon}^{i}\left(\frac{x}{|x|}\right) \\
& +2|x| \Delta v_{\varepsilon}^{i}+|x| x \cdot \nabla \Delta v_{\varepsilon}^{i}+O\left(|x|^{3} \log ^{2}|x|\right)
\end{aligned}
$$

while

$$
\frac{x}{|x|} \cdot \nabla\left(-2 K_{g} u_{\varepsilon}^{i}\right)=4 \frac{\beta_{i}^{2}}{\alpha_{i}^{2}}|x| v_{\varepsilon}^{i}+2 \frac{\beta_{i}^{2}}{\alpha_{i}^{2}}|x| x \cdot \nabla v_{\varepsilon}^{i}
$$

so on $\partial B_{\varepsilon}\left(p_{j}\right)$, we have

$$
\begin{aligned}
\partial_{\nu}\left(\mathscr{L}_{g} u_{\varepsilon}^{i}\right)= & 4 \varepsilon \frac{\beta_{i}^{2}}{\alpha_{i}^{2}} v-4 \frac{\beta_{i}^{2}}{\alpha_{i}^{2}} \varepsilon^{2}\left(\frac{1}{2}+3 \log \left(\frac{1}{\varepsilon}\right)\right) \partial_{\nu} v \\
& -4 \varepsilon\left(1+\frac{\beta_{i}^{2}}{\alpha_{i}^{2}} \log \left(\frac{1}{\varepsilon}\right)\right)\left(\frac{x}{\varepsilon}\right)^{t} D^{2} v_{\varepsilon}^{i}\left(\frac{x}{\varepsilon}\right)+2 \varepsilon \Delta v_{\varepsilon}^{i} \\
& +\varepsilon^{2} \partial_{\nu} \Delta v_{\varepsilon}^{i}+O\left(\varepsilon^{3} \log ^{2} \varepsilon\right) .
\end{aligned}
$$

Since can neglect all terms containing derivatives of $v$, we can replace $v$ by $v\left(p_{i}\right)$ and replace $\partial_{\nu} v$ by 0 , which gives

$$
\begin{aligned}
& u_{\varepsilon}^{i} \partial_{\nu}\left(\mathscr{L}_{g} u_{\varepsilon}^{i}\right)-\left(\partial_{\nu} u_{\varepsilon}^{i}\right) \mathscr{L}_{g} u_{\varepsilon}^{i}=\left(\frac{\alpha_{i}^{2}}{\varepsilon^{2}}+\beta_{i}^{2} \log ^{2}(\varepsilon)\right) v\left(p_{i}\right) \\
& \left(4 \varepsilon \frac{\beta_{i}^{2}}{\alpha_{i}^{2}} v\left(p_{i}\right)-4 \varepsilon\left(1+\frac{\beta_{i}^{2}}{\alpha_{i}^{2}} \log \left(\frac{1}{\varepsilon}\right)\right)\left(\frac{x}{\varepsilon}\right)^{t} D^{2} v_{\varepsilon}^{i}\left(\frac{x}{\varepsilon}\right)\right. \\
& \left.\quad+2 \varepsilon \Delta v_{\varepsilon}^{i}\right)+2\left(\frac{\alpha_{i}^{2}}{\varepsilon^{3}}+\frac{\beta_{i}^{2}}{\varepsilon} \log \left(\frac{1}{\varepsilon}\right)\right) v\left(p_{i}\right)\left(\left(4+2 \frac{\beta_{i}^{2}}{\alpha_{i}^{2}} \varepsilon^{2}\right) v\left(p_{i}\right)+\varepsilon^{2} \Delta v_{\varepsilon}^{i}\right)+O\left(\log ^{2} \varepsilon\right) \\
& =8 \frac{\alpha_{i}^{2}}{\varepsilon^{3}} v^{2}\left(p_{i}\right)+8 \frac{\beta_{i}^{2}}{\varepsilon} \log \left(\frac{1}{\varepsilon}\right) v^{2}\left(p_{i}\right)+8 \frac{\beta_{i}^{2}}{\varepsilon} v^{2}\left(p_{i}\right)+2 \frac{\alpha_{i}^{2}}{\varepsilon} \Delta v_{\varepsilon}^{i} v\left(p_{i}\right) \\
& +\frac{\alpha_{i}^{2}}{\varepsilon^{2}}\left(-4 \varepsilon\left(1+\frac{\beta_{i}^{2}}{\alpha_{i}^{2}} \log \left(\frac{1}{\varepsilon}\right)\right)\left(\frac{x}{\varepsilon}\right)^{t} D^{2} v_{\varepsilon}^{i}\left(\frac{x}{\varepsilon}\right)+2 \varepsilon \Delta v_{\varepsilon}^{i}\right) v\left(p_{i}\right)+O\left(\log ^{2} \varepsilon\right) .
\end{aligned}
$$


Furthermore, we note that if

$$
D^{2} v_{\varepsilon}^{i}=\left(\begin{array}{ll}
a_{1,1} & a_{1,2} \\
a_{2,1} & a_{2,2}
\end{array}\right)+O(\varepsilon)
$$

then

$$
\begin{aligned}
& \int_{\partial B_{\varepsilon}\left(p_{i}\right)} x^{t} D^{2} v_{\varepsilon}^{i} x d \mathscr{H}^{1} \\
& \quad=\varepsilon^{3} \int_{0}^{2 \pi}\left(a_{1,1} \cos ^{2}(\theta)+a_{2,2} \sin ^{2}(\theta)+\frac{a_{1,2}+a_{2,1}}{2} \sin (2 \theta)\right) d \theta+O\left(\varepsilon^{4}\right) \\
& \quad=\pi\left(a_{1,1}+a_{2,2}\right) \varepsilon^{3}+O\left(\varepsilon^{4}\right)
\end{aligned}
$$

while

$$
\int_{\partial B_{\varepsilon}\left(p_{i}\right)} \Delta v_{\varepsilon}^{i}=2 \pi\left(a_{1,1}+a_{2,2}\right) \varepsilon+O\left(\varepsilon^{2}\right)
$$

so if we write $\delta_{i}=a_{1,1}+a_{2,2}$, we have

$$
\begin{gathered}
\int_{\partial B_{\varepsilon}\left(p_{i}\right)} \frac{\alpha_{i}^{2}}{\varepsilon^{2}}\left(-4 \varepsilon\left(1+\frac{\beta_{i}^{2}}{\alpha_{i}^{2}} \log \left(\frac{1}{\varepsilon}\right)\right)\left(\frac{x}{\varepsilon}\right)^{t} D^{2} v_{\varepsilon}^{i}\left(\frac{x}{\varepsilon}\right)+2 \varepsilon \Delta v_{\varepsilon}^{i}\right) d \mathscr{H}^{1} \\
=-4\left(\alpha_{i}^{2}+\beta_{i}^{2} \log \left(\frac{1}{\varepsilon}\right)\right) \pi \delta_{i}+2 \alpha_{i}^{2} \cdot 2 \pi \delta_{i}+O(\varepsilon \log \varepsilon) \\
=-4 \pi \beta_{i}^{2} \log \left(\frac{1}{\varepsilon}\right) \delta_{i}+O(\varepsilon \log \varepsilon)
\end{gathered}
$$

we obtain finally

$$
\begin{aligned}
Q_{\varepsilon}\left(u_{\varepsilon}^{i}\right)= & \frac{1}{2} \int_{\partial B_{\varepsilon}\left(p_{i}\right)} u_{\varepsilon}^{i} \partial_{v}\left(\mathscr{L}_{g} u_{\varepsilon}^{i}\right)-\left(\partial_{v} u_{\varepsilon}^{i}\right) \mathscr{L}_{g} u_{\varepsilon}^{i} d \mathscr{H}^{1} \\
= & \frac{8 \pi \alpha_{i}^{2}}{\varepsilon^{2}} v^{2}\left(p_{i}\right)+8 \pi \beta_{i}^{2} \log \left(\frac{1}{\varepsilon}\right) v^{2}\left(p_{i}\right)+8 \pi \beta_{i}^{2} v^{2}\left(p_{i}\right) \\
& -2 \pi \beta_{i}^{2} \log \left(\frac{1}{\varepsilon}\right) \delta_{i} v\left(p_{i}\right)+2 \pi \beta_{i}^{2} \delta_{i} v\left(p_{i}\right)+O(\varepsilon \log \varepsilon) .
\end{aligned}
$$

Now thanks to the asymptotic behaviour of $\left\{v_{\varepsilon}^{i}\right\}_{1 \leq i \leq n}$, we know that $B_{\varepsilon}\left(u_{\varepsilon}, u_{\varepsilon}^{i}\right)$, and $B_{\varepsilon}\left(u_{\varepsilon}^{i}, u_{\varepsilon}^{j}\right)$ are bounded terms, so for the energy to be finite, we must have

$$
Q_{\varepsilon}\left(u_{\varepsilon}^{i}\right)=\frac{8 \pi \alpha_{i}^{2}}{\varepsilon^{2}} v^{2}\left(p_{i}\right)+24 \pi \beta_{i}^{2} \log \left(\frac{1}{\varepsilon}\right)+O(1)
$$

which imposes

$$
\delta_{i}=-8 v\left(p_{i}\right)
$$

and we get

$$
Q_{\varepsilon}\left(u_{\varepsilon}^{i}\right)=\frac{8 \pi \alpha_{i}^{2}}{\varepsilon^{2}} v^{2}\left(p_{i}\right)-8 \pi \beta_{i}^{2} \log \left(\frac{1}{\varepsilon}\right) v^{2}\left(p_{i}\right)-8 \pi \beta_{i}^{2} v^{2}\left(p_{i}\right)+O\left(\varepsilon \log ^{2} \varepsilon\right) .
$$

Step 2: Estimation of $B_{\varepsilon}\left(u_{\varepsilon}^{i}, u_{\varepsilon}^{j}\right)$ for $i \neq j$. 
For all $1 \leq i, j \leq n$, and $k \neq i, j$ we have by Theorem 5.5

$$
\int_{\partial B_{\varepsilon}\left(p_{k}\right)} u_{\varepsilon}^{i} \partial_{\nu}\left(\mathscr{L}_{g} u_{\varepsilon}^{j}\right) d \mathscr{H}^{1}=\int_{\partial B_{\varepsilon}\left(p_{k}\right)} O\left(\frac{1}{|x|}\right) O\left(|x|^{2}\right) d \mathscr{H}^{1}=O\left(\varepsilon^{2} \log \varepsilon\right)
$$

and likewise

$$
\int_{\partial B_{\varepsilon}\left(p_{k}\right)} \partial_{\nu}\left(u_{\varepsilon}^{i}\right) \mathscr{L}_{g} u_{\varepsilon}^{j} d \mathscr{H}^{1}=\int_{\partial B_{\varepsilon}\left(p_{k}\right)} O\left(\frac{1}{|x|^{2}}\right) O\left(|x|^{3}\right) d \mathscr{H}^{1}=O\left(\varepsilon^{2}\right)
$$

therefore

$$
\sum_{k \neq i, j} \int_{\partial B_{\varepsilon}\left(p_{k}\right)}\left(u_{\varepsilon}^{i} \partial_{\nu}\left(\mathscr{L}_{g} u_{\varepsilon}^{j}\right)-\partial_{\nu}\left(u_{\varepsilon}^{i}\right) \mathscr{L}_{g} u_{\varepsilon}^{j}\right) d \mathscr{H}^{1}=O\left(\varepsilon^{2} \log \varepsilon\right)
$$

So we need only to consider the boundary integrals for $B_{\varepsilon}\left(p_{i}\right)$ and $B_{\varepsilon}\left(p_{j}\right)$. We have up to $O\left(\varepsilon^{3} \log \varepsilon\right)$ error terms by (6.22)

$$
\begin{aligned}
\int_{\Sigma_{\varepsilon}} \mathscr{L}_{g} u_{\varepsilon}^{i} \mathscr{L}_{g} u_{\varepsilon}^{j}= & \int_{\partial B_{\varepsilon}\left(p_{i}\right)}\left(u_{\varepsilon}^{i} \partial_{\nu}\left(\mathscr{L}_{g} u_{\varepsilon}^{j}\right)-\left(\partial_{\nu} u_{\varepsilon}^{i}\right) \mathscr{L}_{g} u_{\varepsilon}^{j}\right) d \mathscr{H}^{1} \\
& +\int_{\partial B_{\varepsilon}\left(p_{j}\right)}\left(u_{\varepsilon}^{i} \partial_{\nu}\left(\mathscr{L}_{g} u_{\varepsilon}^{j}\right)-\left(\partial_{\nu} u_{\varepsilon}^{i}\right) \mathscr{L}_{g} u_{\varepsilon}^{j}\right) d \mathscr{H}^{1}
\end{aligned}
$$

and

$$
\begin{aligned}
& \int_{\partial B_{\varepsilon}\left(p_{i}\right)}\left(u_{\varepsilon}^{i} \partial_{\nu}\left(\mathscr{L}_{g} u_{\varepsilon}^{j}\right)-\left(\partial_{\nu} u_{\varepsilon}^{i}\right) \mathscr{L}_{g} u_{\varepsilon}^{j}\right) d \mathscr{H}^{1} \\
& \quad=\int_{\partial B_{\varepsilon}\left(p_{i}\right)} O\left(\frac{1}{|x|^{2}}\right) O\left(|x|^{2}\right)-O\left(\frac{1}{|x|^{3}}\right) O\left(|x|^{3}\right) d \mathscr{H}^{1}=O(\varepsilon)
\end{aligned}
$$

so by symmetry, we have

$$
\begin{aligned}
\frac{1}{2} B_{\varepsilon}\left(u_{\varepsilon}^{i}, u_{\varepsilon}^{j}\right) & =\frac{1}{2} \int_{\partial B_{\varepsilon}\left(p_{j}\right)}\left(u_{\varepsilon}^{i} \partial_{\nu}\left(\mathscr{L}_{g} u_{\varepsilon}^{j}\right)-\left(\partial_{\nu} u_{\varepsilon}^{i}\right) \mathscr{L}_{g} u_{\varepsilon}^{j}\right) d \mathscr{H}^{1}+O(\varepsilon) \\
& =\frac{1}{2} \int_{\partial B_{\varepsilon}\left(p_{i}\right)}\left(u_{\varepsilon}^{j} \partial_{\nu}\left(\mathscr{L}_{g} u_{\varepsilon}^{i}\right) d \mathscr{H}^{1}-\left(\partial_{\nu} u_{\varepsilon}^{i}\right) \mathscr{L}_{g} u_{\varepsilon}^{i}\right)+O(\varepsilon) .
\end{aligned}
$$

From now on, we find useful to use complex notations. Recall the expansion on $\partial B_{\varepsilon}\left(p_{j}\right)$

$$
u_{\varepsilon}^{i}=\operatorname{Re}\left(\frac{c_{i, j}}{z}+d_{i, j} \frac{\bar{z}}{z}\right)+a_{i, j} \log |z|+b_{i, j}+O(|z|) .
$$

Furthermore, as

$$
|\vec{\Phi}|^{2}=\frac{\alpha_{j}^{2}}{|z|^{2}}+\beta_{i}^{2} \log ^{2}|z|+O(|z| \log |z|)
$$

we have

$$
u_{\varepsilon}^{j}=|\vec{\Phi}|^{2}\left(v\left(p_{j}\right)+\operatorname{Re}(\gamma z)+O\left(|z|^{2}\right)\right)=\frac{\alpha_{j}^{2}}{|z|^{2}}\left(v\left(p_{j}\right)+\operatorname{Re}(\gamma z)+O\left(|z|^{2} \log ^{2}|z|\right)\right) .
$$

Furthermore, we have

$$
e^{2 \lambda}=\frac{\alpha_{i}^{2}}{|z|^{4}}(1+O(|z|))
$$


so we deduce that

$$
\Delta_{g} u_{\varepsilon}^{j}=4 v\left(p_{j}\right)+O\left(|z|^{2} \log ^{2}|z|\right) .
$$

Furthermore, as $K_{g}=O\left(|z|^{4}\right)$, we have also

$$
K_{g} u_{\varepsilon}^{j}=O\left(|z|^{2}\right),
$$

so we get

$$
\begin{aligned}
& \mathscr{L}_{g} u_{\varepsilon}^{j}=4 v\left(p_{j}\right)+O\left(|z|^{2} \log |z|\right) \\
& \partial_{v}\left(\mathscr{L}_{g} u_{\varepsilon}^{j}\right)=O\left(|z| \log ^{2}|z|\right),
\end{aligned}
$$

so we recover a weak form of (6.18) (however sufficient for our purpose here). Now we note that

$$
\begin{aligned}
& \int_{\partial B_{\varepsilon}\left(p_{j}\right)} u_{\varepsilon}^{i} \partial_{\nu}\left(\mathscr{L}_{g} u_{\varepsilon}^{j}\right) d \mathscr{H}^{1} \\
& \quad=\int_{\partial B_{\varepsilon}\left(p_{j}\right)}\left(\operatorname{Re}\left(\frac{c_{\varepsilon}^{i}}{z}\right)+O(\log |z|)\right) O\left(|z| \log ^{2}|z|\right) d \mathscr{H}^{1}=O\left(\varepsilon \log ^{2} \varepsilon\right) .
\end{aligned}
$$

Now, notice that for all smooth $\varphi: B(0,1) \rightarrow \mathbb{R}$, we have

$$
\begin{aligned}
\partial_{\nu} \varphi & =\frac{x_{1}}{|x|} \cdot \partial_{x_{1}} \varphi+\frac{x_{2}}{|x|} \cdot \partial_{x_{2}} \varphi \\
& =\frac{1}{|z|}\left(\frac{(z+\bar{z})}{2}(\partial+\bar{\partial}) \varphi+\frac{(z-\bar{z})}{2 i} i(\partial-\bar{\partial}) \varphi\right)=\frac{1}{|z|}(z \partial \varphi+\bar{z} \bar{\partial} \varphi)=\frac{2}{|z|} \operatorname{Re}\left(z \partial_{z} \varphi\right) .
\end{aligned}
$$

Therefore, we have (as $\bar{z} / z$ has no radial component)

$$
\partial_{\nu} u_{\varepsilon}^{i}=-\frac{2}{|z|} \operatorname{Re}\left(\frac{c_{i, j}}{z}\right)+\frac{a_{i, j}}{|z|}+O(1),
$$

while

$$
\mathscr{L}_{g} u_{\varepsilon}^{j}=4 v\left(p_{j}\right)+O\left(|z|^{2} \log |z|\right),
$$

therefore

$$
\begin{aligned}
\int_{\partial B_{\varepsilon}\left(p_{j}\right)} \partial_{\nu} u_{\varepsilon}^{i} \mathscr{L}_{g} u_{\varepsilon}^{j} d \mathscr{H}^{1}= & \int_{\partial B_{\varepsilon}\left(p_{j}\right)}\left(-\frac{c_{i, j}}{2|z| z}-\frac{\overline{c_{i, j}}}{2|z| \bar{z}}+\frac{a_{i, j}}{|z|}+O(\log |z|)\right) \\
& \left(4 v\left(p_{j}\right)+O\left(|z|^{2} \log |z|\right)\right) d \mathscr{H}^{1} \\
= & 8 \pi a_{i, j} v\left(p_{j}\right)+O\left(\varepsilon \log ^{2} \varepsilon\right) .
\end{aligned}
$$

Therefore by symmetry

$$
\frac{1}{2} B_{\varepsilon}\left(u_{\varepsilon}^{i}, u_{\varepsilon}^{j}\right)=-4 \pi a_{i, j} v\left(p_{j}\right)+O\left(\varepsilon \log ^{2} \varepsilon\right)=-4 \pi a_{j, i}\left(p_{i}\right)+O\left(\varepsilon \log ^{2} \varepsilon\right)
$$

so

$$
a_{\varepsilon}^{i}\left(p_{j}\right) v\left(p_{j}\right)=a_{\varepsilon}^{j} v\left(p_{i}\right)
$$

therefore there exists $\lambda_{i, j} \in \mathbb{R}$ such that $a_{i, j}=\lambda_{i, j} v\left(p_{i}\right), a_{j, i}=\lambda_{i, j} v\left(p_{j}\right)$ and we deduce that

$$
\frac{1}{2} B_{\varepsilon}\left(p_{i}, p_{j}\right)=-4 \pi \lambda_{i, j} v\left(p_{i}\right) v\left(p_{j}\right)+O(\varepsilon \log \varepsilon) .
$$


We note that these notations imply that for $r>0$ small enough, for all $1 \leq i \leq n$, for all $j \neq i$ we have on any conformal chart $D^{2} \rightarrow \bar{B}_{r}\left(p_{j}\right)$

$$
u_{\varepsilon}^{i}(z)=\operatorname{Re}\left(\frac{c_{i, j}}{z}+d_{i, j} \frac{\bar{z}}{z}\right)+\lambda_{i, j} v\left(p_{i}\right) \log |z|+b_{i, j}+O(|z|) .
$$

Step 3: Estimation of $B_{\varepsilon}\left(u_{\varepsilon}, u_{\varepsilon}^{i}\right)$ for $1 \leq i \leq n$.

We note that the boundary conditions imply that for all $1 \leq i \leq n$, we have on $\partial B_{\varepsilon}\left(p_{i}\right)$ (for some $\gamma_{i}, \tilde{\gamma}_{i} \in \mathbb{C}$ and $b_{i} \in \mathbb{R}$ )

$$
\begin{aligned}
u_{\varepsilon} & =u-\sum_{j=1}^{n} u_{\varepsilon}^{j}=-\sum_{j \neq i} u_{\varepsilon}^{j}=\operatorname{Re}\left(\frac{\gamma_{i}}{z}+\tilde{\gamma}_{i} \frac{\bar{z}}{z}\right)-\sum_{j \neq i} \lambda_{i, j} v\left(p_{j}\right) \log |z|+b_{i}+O(|z|) \\
& =\operatorname{Re}\left(\frac{\gamma_{i}}{z}\right)-\sum_{j \neq i} \lambda_{i, j} v\left(p_{j}\right) \log |z|+O(1) \\
\partial_{\nu} u_{\varepsilon} & =-\frac{1}{\varepsilon} \operatorname{Re}\left(\frac{\gamma_{i}}{z}\right)-\frac{1}{\varepsilon} \sum_{j \neq i} \lambda_{i, j} v\left(p_{j}\right)+O(1),
\end{aligned}
$$

where we used $\partial_{\nu}\left(\widetilde{\gamma_{i}} \frac{\bar{z}}{z}\right)=0$. As by the Remark 5.7 for all $j \neq i$, we have on $\partial_{\nu} B_{\varepsilon}\left(p_{j}\right)$

$$
\begin{aligned}
& \mathscr{L}_{g} u_{\varepsilon}^{i}=O\left(\varepsilon^{2}\right) \\
& \partial_{\nu}\left(\mathscr{L}_{g} u_{\varepsilon}^{i}\right)=O(\varepsilon)
\end{aligned}
$$

we deduce that

$$
\int_{\partial B_{\varepsilon}\left(p_{j}\right)} u_{\varepsilon} \partial_{\nu}\left(\mathscr{L}_{g} u_{\varepsilon}^{i}\right)-\partial_{\nu} u_{\varepsilon} \mathscr{L}_{g} u_{\varepsilon}^{i} d \mathscr{H}^{1}=O(\varepsilon),
$$

and as on $\partial B_{\varepsilon}\left(p_{i}\right)$

$$
\begin{aligned}
& \mathscr{L}_{g} u_{\varepsilon}^{i}=4 v\left(p_{i}\right)+O\left(\varepsilon^{2} \log ^{2} \varepsilon\right) \\
& \partial_{\nu}\left(\mathscr{L}_{g} u_{\varepsilon}^{i}\right)=O\left(\varepsilon \log ^{2} \varepsilon\right)
\end{aligned}
$$

we have

$$
\int_{\partial B_{\varepsilon}\left(p_{i}\right)} u_{\varepsilon} \partial_{\nu}\left(\mathscr{L}_{g} u_{\varepsilon}^{i}\right) d \mathscr{H}^{1}=\int_{\partial B_{\varepsilon}\left(p_{i}\right)} O\left(\log ^{2} \varepsilon\right) d \mathscr{H}^{1}=O\left(\varepsilon \log ^{2} \varepsilon\right)
$$

so finally, as $\mathscr{L}_{g}^{2} u_{\varepsilon}^{i}=0$ on $\Sigma_{\varepsilon}$

$$
\begin{aligned}
B_{\varepsilon}\left(u_{\varepsilon}, u_{\varepsilon}^{i}\right)= & \int_{\Sigma_{\varepsilon}} \mathscr{L}_{g} u_{\varepsilon} \mathscr{L}_{g} u_{\varepsilon}^{i} d \operatorname{vol}_{g} \\
& =\int_{\Sigma_{\varepsilon}} u_{\varepsilon} \mathscr{L}_{g}^{2} u_{\varepsilon}^{i} d \operatorname{vol}_{g}+\sum_{j=1}^{n} \int_{\partial B_{\varepsilon}\left(p_{j}\right)} u_{\varepsilon} \partial_{\nu}\left(\mathscr{L}_{g} u_{\varepsilon}^{i}\right)-\partial_{\nu} u_{\varepsilon} \mathscr{L}_{g} u_{\varepsilon}^{i} d \mathscr{H}^{1} \\
& =\int_{\partial B_{\varepsilon}\left(p_{i}\right)}-\frac{1}{\varepsilon}\left(-\operatorname{Re}\left(\frac{\gamma_{i}}{z}\right)-\sum_{j \neq i} \lambda_{i, j} v\left(p_{j}\right)+O(\varepsilon)\right)\left(4 v\left(p_{i}\right)\right. \\
& +O(\varepsilon)) d \mathscr{H}^{1}+O\left(\varepsilon \log ^{2} \varepsilon\right)
\end{aligned}
$$




$$
=8 \pi \sum_{j \neq i} \lambda_{i, j} v\left(p_{i}\right) v\left(p_{j}\right)+O\left(\varepsilon \log ^{2} \varepsilon\right),
$$

where we used by obvious symmetry

$$
\int_{\partial B(0, \varepsilon)} \operatorname{Re}\left(\frac{\gamma_{i}}{z}\right) d \mathscr{H}^{1}=0 .
$$

Therefore for all $1 \leq i \leq n$, one has

$$
B_{\varepsilon}\left(u_{\varepsilon}, u_{\varepsilon}^{i}\right)=8 \pi \sum_{j \neq i} \lambda_{i, j} v\left(p_{i}\right) v\left(p_{j}\right)+O(\varepsilon \log \varepsilon) .
$$

Conclusion: We have finally by (6.17), (6.20), (6.23), (6.24)

$$
\begin{aligned}
Q_{\varepsilon}(u)= & Q_{\varepsilon}\left(u_{\varepsilon}\right)+8 \pi \sum_{i=1}^{n} \frac{\alpha_{i}^{2}}{\varepsilon^{2}} v^{2}\left(p_{i}\right)+24 \pi \sum_{j=1}^{m} \beta_{j}^{2} \log \left(\frac{1}{\varepsilon}\right) v^{2}\left(p_{j}\right)-8 \pi \sum_{j=1}^{m} \beta_{j}^{2} v^{2}\left(p_{j}\right) \\
& +2 \sum_{i=1}^{n}\left(4 \pi \sum_{j \neq i} \lambda_{i, j} v\left(p_{i}\right) v\left(p_{j}\right)\right)-4 \pi \sum_{i \neq j} \lambda_{i, j} v\left(p_{i}\right) v\left(p_{j}\right)+O\left(\varepsilon \log ^{2} \varepsilon\right) \\
= & Q_{\varepsilon}\left(u_{\varepsilon}\right)+8 \pi \sum_{i=1}^{n} \frac{\alpha_{i}^{2}}{\varepsilon^{2}} v^{2}\left(p_{i}\right)+24 \pi \sum_{j=1}^{m} \beta_{j}^{2} \log \left(\frac{1}{\varepsilon}\right) v^{2}\left(p_{j}\right)-8 \pi \sum_{i=1}^{n} \beta_{j}^{2} v^{2}\left(p_{j}\right) \\
& +4 \pi \sum_{i \neq j} \lambda_{i, j} v\left(p_{i}\right) v\left(p_{j}\right)+O\left(\varepsilon \log ^{2} \varepsilon\right)
\end{aligned}
$$

and finally

$$
\begin{aligned}
Q(u)= & \lim _{\varepsilon \rightarrow 0}\left(Q_{\varepsilon}(u)-8 \pi \sum_{i=1}^{n} \frac{\alpha_{i}^{2}}{\varepsilon^{2}} v^{2}\left(p_{i}\right)-24 \pi \sum_{j=1}^{m} \beta_{j}^{2} \log \left(\frac{1}{\varepsilon}\right) v^{2}\left(p_{j}\right)\right. \\
& \left.+16 \pi \sum_{j=1}^{m} \beta_{j}^{2} v^{2}\left(p_{j}\right)\right) \\
= & Q\left(u_{0}\right)+8 \pi \sum_{j=1}^{m} \beta_{j}^{2} v^{2}\left(p_{j}\right)+4 \pi \sum_{i \neq j} \lambda_{i, j} v\left(p_{i}\right) v\left(p_{j}\right),
\end{aligned}
$$

which concludes the proof, as the last claim follows from the fact that

$$
\begin{aligned}
& \int_{\partial B_{\varepsilon}\left(p_{i}\right)} u_{\varepsilon} \partial_{\nu}\left(\mathscr{L}_{g} u_{\varepsilon}\right)-\partial_{\nu} u_{\varepsilon}\left(\mathscr{L}_{g} u_{\varepsilon}\right) d \mathscr{H}^{1} \\
& \quad=\int_{\partial B_{\varepsilon}\left(p_{i}\right)} O(\log \varepsilon) O\left(\varepsilon^{2}\right)-O\left(\frac{1}{\varepsilon}\right) O\left(\varepsilon^{3}\right) d \mathscr{H}^{1}=O\left(\varepsilon^{3} \log \varepsilon\right) .
\end{aligned}
$$

which concludes the proof of the theorem.

We deduce from the preceding theorem an improvement of Proposition 5.5

Corollary 6.7 For all $1 \leq i \leq n$, there exists $\lambda_{i, j} \in \mathbb{R}$ such that for all $j \neq i$, for all $0<\varepsilon<\varepsilon_{0}$, on every complex chart around $p_{j}$ there exists $c_{i, j}, d_{i, j} \in \mathbb{C}$ and $b_{i, j} \in \mathbb{R}$ such 
that

$$
u_{\varepsilon}^{i}(z)=\operatorname{Re}\left(\frac{c_{i, j}}{z}+d_{i, j} \frac{\bar{z}}{z}\right)+\lambda_{i, j} v\left(p_{i}\right) \log |z|+b_{i, j}+O(|z| \log |z|) .
$$

\section{Equality of the Morse index for inversions of minimal surfaces with embedded ends}

Theorem 7.1 Let $\Sigma$ be a closed Riemann surface, $\vec{\Phi}: \Sigma \backslash\left\{p_{1}, \ldots, p_{n}\right\} \rightarrow \mathbb{R}^{3}$ be a complete minimal surface with finite total curvature and embedded ends, and $\vec{\Psi}: \Sigma \rightarrow \mathbb{R}^{3}$ be its inversion. Assume that $0 \leq m \leq n$ is fixed such that $p_{1}, \ldots, p_{m}$ are catenoid ends, while $p_{m+1}, \ldots, p_{n}$ are planar ends, and for all $1 \leq j \leq m$, let $\beta_{j}=\left|\operatorname{Flux}\left(\vec{\Phi}, p_{j}\right)\right| \in \mathbb{R}_{+}^{*}$ be the norm of the flux of $\vec{\Phi}$ at $p_{j}$. Let $\Lambda(\vec{\Psi}) \in \operatorname{Sym}_{n}(\mathbb{R})$ be the symmetric matrix defined (see Corollary 6.7) by

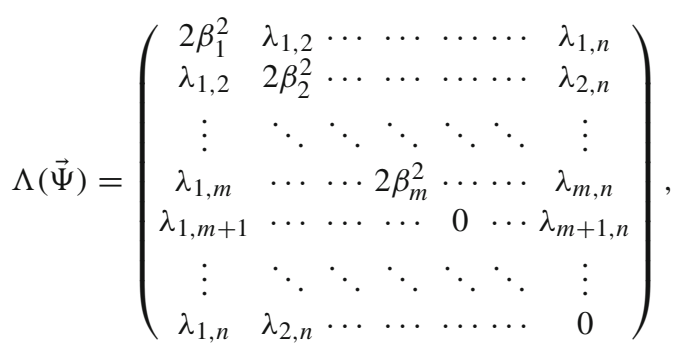

Then for all $a=\left(a_{1}, \ldots, a_{n}\right) \in \mathbb{R}^{n}$, there exists $v=v_{a} \in \mathrm{W}^{2,2}(\Sigma)$ such that $\left(v\left(p_{1}\right), \ldots, v\left(p_{n}\right)\right)=\left(a_{1}, \ldots, a_{n}\right)$ and if $\vec{v}_{0}=-v_{0} \vec{n}_{\vec{\Psi}}+2 \operatorname{Re}\left(\alpha_{0} \otimes \partial \vec{\Psi}\right)$, where $\alpha_{0}$ is given by the proof of Theorem 6.2, we have

$$
Q_{\vec{\Psi}}\left(\vec{v}_{0}\right)=8 \pi \sum_{i=1}^{m} \beta_{j}^{2} v^{2}\left(p_{j}\right)+4 \pi \sum_{1 \leq i, j \leq n} \lambda_{i, j} v\left(p_{i}\right) v\left(p_{j}\right) .
$$

Therefore, we have

$$
\operatorname{Ind}_{W}(\vec{\Psi})=\operatorname{Ind} \Lambda(\vec{\Psi}) \leq n-1,
$$

where the index Ind of a matrix is the number of its negative eigenvalues.

Proof Let $v \in C^{2}(\Sigma)$ be such that $v\left(p_{i}\right) \neq 0$ and consider $u_{0}=\sum_{i=1}^{n} u_{0}^{i}=|\vec{\Phi}|^{2} \sum_{i=1}^{n} v_{0}^{i}$ obtained in Theorem 5.9. We assume for simplicity that the end is planar, as the computation for a catenoid end would be identical up (notice that we can also assume the variation to be normal in the catenoidal case since the tangential part vanishes in the residue). Recall now that in the chart $U_{i}$ around $p_{i}$, we have for all $j \neq i$

$$
\begin{aligned}
& |\vec{\Phi}|^{2}=\frac{\alpha_{i}^{2}}{|z|^{2}}\left(1+O\left(|z|^{2}\right)\right) \\
& u_{0}^{i}=\frac{\alpha_{i}^{2}}{|z|^{2}}\left(v\left(p_{i}\right)+O(|z|)\right) \quad u_{0}^{j}=\operatorname{Re}\left(\frac{\gamma_{i, j}}{z}+\tilde{\gamma}_{i, j} \frac{\bar{z}}{z}\right)+\lambda_{i, j} v\left(p_{j}\right) \log |z|+\mu_{i, j}+O(|z|) .
\end{aligned}
$$

Therefore, we find

$$
v_{0}^{i}=v\left(p_{i}\right)+O(|z|)
$$




$$
v_{0}^{j}=\operatorname{Re}\left(\frac{\gamma_{i, j}}{\alpha_{i}^{2}} z+\frac{\overline{\tilde{\gamma}_{i, j}}}{\alpha_{i}^{2}} z^{2}\right)+\frac{\lambda_{i, j}}{\alpha_{i}^{2}}|z|^{2} \log |z|+\frac{\mu_{i, j}}{\beta_{i}^{2}}|z|^{2}+O\left(|z|^{3}\right) .
$$

Furthermore, as $v_{0}^{i}$ is regular at $p_{i}$, this implies if $u_{0}=|\vec{\Phi}|^{2} v_{0}$ that there exists $\gamma_{0} \in \mathbb{R}$ and $\zeta_{0}, \zeta_{1} \in \mathbb{C}$ such that

$$
v_{0}=v\left(p_{i}\right)+2 \operatorname{Re}\left(\zeta_{0} z+\zeta_{1} z^{2}\right)+\gamma_{0}|z|^{2}+\sum_{j \neq i} \frac{\lambda_{i, j}}{\alpha_{i}^{2}} v\left(p_{j}\right)|z|^{2} \log |z|+O\left(|z|^{3}\right) .
$$

Therefore, one needs to compute the renormalised energy for variations not only $C^{2}$ but also of the form given by (7.3).

Let $v \in \mathrm{W}^{2,2}(\Sigma)$ be such that

$$
v=v\left(p_{i}\right)+2 \operatorname{Re}\left(\zeta_{0} z+\zeta_{1} z^{2}\right)+\gamma_{0}|z|^{2}+\gamma_{1}|z|^{2} \log |z|+O\left(|z|^{3}\right) .
$$

We will now compute $Q_{\vec{\Psi}}(v)$ for the variation $v$ in (7.4). Now, recall that at a planar end there exists $\alpha_{i}^{2}>0$ and $\alpha_{0} \in \mathbb{C}$ such that

$$
|\vec{\Phi}|^{2}=\frac{\alpha_{i}^{2}}{|z|^{2}}\left(1+2 \operatorname{Re}\left(\alpha_{0} z^{2}\right)+O\left(|z|^{3}\right)\right) .
$$

As $\vec{\Phi}$ is minimal, we deduce that

$$
g=e^{2 \lambda}|d z|^{2}=\partial \bar{\partial}|\vec{\Phi}|^{2}=\frac{\alpha_{i}^{2}}{|z|^{4}}\left(1-2 \operatorname{Re}\left(\alpha_{0} z^{2}\right)+O\left(|z|^{3}\right)\right) .
$$

Therefore, we have

$$
\begin{aligned}
u & =|\vec{\Phi}|^{2} v=\frac{\alpha_{i}^{2}}{|z|^{2}}\left(v\left(p_{i}\right)+2 \operatorname{Re}\left(\zeta_{0} z+\left(\alpha_{0} v\left(p_{i}\right)+\zeta_{1}\right) z^{2}\right)\right)+\alpha_{i}^{2} \gamma_{0}+\alpha_{i}^{2} \gamma_{1} \log |z|+O(|z|) \\
& =\frac{\alpha_{i}^{2}}{|z|^{2}}\left(v\left(p_{i}\right)+2 \operatorname{Re}\left(\zeta_{0} z+\zeta_{2} z^{2}\right)\right)+\alpha_{i}^{2} \gamma_{0}+\alpha_{i}^{2} \gamma_{1} \log |z|+O(|z|)
\end{aligned}
$$

and (as $|z|^{-2} \operatorname{Re}\left(\zeta_{0} z\right)=\operatorname{Re}\left(\overline{\zeta_{0}} z^{-1}\right)$ is harmonic)

$$
\begin{aligned}
\Delta u & =\frac{4 \alpha_{i}^{2}}{|z|^{4}}\left(v\left(p_{i}\right)-2 \operatorname{Re}\left(\zeta_{2} z^{2}\right)+O\left(|z|^{3}\right)\right) \\
\Delta_{g} u & =e^{-2 \lambda} \Delta u=\frac{|z|^{4}}{\alpha_{i}^{2}}\left(1+2 \operatorname{Re}\left(\alpha_{0} z^{2}\right)+O\left(|z|^{3}\right)\right) \times \frac{4 \alpha_{i}^{2}}{|z|^{4}}\left(v\left(p_{i}\right)-2 \operatorname{Re}\left(\zeta_{2} z^{2}\right)+O\left(|z|^{3}\right)\right) \\
& =4 v\left(p_{i}\right)+2 \operatorname{Re}\left(\left(\alpha_{0} v\left(p_{i}\right)-\zeta_{2}\right) z^{2}\right)+O\left(|z|^{3}\right) \\
& =4 v\left(p_{i}\right)+2 \operatorname{Re}\left(\zeta_{3} z^{2}\right)+O\left(|z|^{3}\right)
\end{aligned}
$$

Now, we have

$$
\begin{aligned}
\partial u & =-\frac{\alpha_{i}^{2}}{z|z|^{2}}\left(v\left(p_{i}\right)+\overline{\zeta_{0}} \bar{z}-\zeta_{2} z^{2}+\bar{\zeta}_{2} \bar{z}^{2}\right) d z+\frac{\alpha_{i}^{2} \gamma_{1}}{2} \frac{d z}{z}+O(1) \\
& =-\frac{\alpha_{i}^{2}}{z|z|^{2}}\left(v\left(p_{i}\right)+\overline{\zeta_{0}} \bar{z}-2 i \operatorname{Im}\left(\zeta_{2} z^{2}\right)\right) d z+\frac{\alpha_{i}^{2} \gamma_{1}}{2} \frac{d z}{z}+O(1)
\end{aligned}
$$

This implies that we have for some $\lambda_{0}, \lambda_{1} \in \mathbb{C}$

$$
\Delta_{g}\left(|\vec{\Phi}|^{2} v\right) \partial\left(|\vec{\Phi}|^{2} v\right)=\Delta_{g} u(\partial u)=-\frac{4 \alpha_{i}^{2}}{z|z|^{2}}\left(v^{2}\left(p_{i}\right)+\overline{\zeta_{0}} v\left(p_{i}\right) \bar{z}+\lambda_{0} z^{2}+\lambda_{1} \bar{z}^{2}\right) d z
$$




$$
+2 \alpha_{i}^{2} \gamma_{1} v\left(p_{i}\right) \frac{d z}{z}+O(1)
$$

This implies that

$$
\operatorname{Im} \int_{\partial B(0, \varepsilon)} \Delta_{g}\left(|\vec{\Phi}|^{2} v\right) \partial\left(|\vec{\Phi}|^{2} v\right)=-\frac{8 \pi \alpha_{i}^{2}}{\varepsilon^{2}} v^{2}\left(p_{i}\right)+4 \pi \alpha_{i}^{2} \gamma_{1} v\left(p_{i}\right)+O(\varepsilon) .
$$

Now, we compute

$$
\begin{aligned}
|\partial u|^{2} & =\left|-\frac{\alpha_{i}^{2}}{z|z|^{2}}\left(v\left(p_{i}\right)+\overline{\zeta_{0}} \bar{z}-2 i \operatorname{Im}\left(\zeta_{2} z^{2}\right)\right) d z+\frac{\alpha_{i}^{2} \gamma_{0}}{2} \frac{d z}{z}+O(1)\right|^{2} \\
& =\left(\frac{\alpha_{i}^{4}}{|z|^{6}}\left(v^{2}\left(p_{i}\right)+2 \operatorname{Re}\left(\zeta_{0} v\left(p_{i}\right) z\right)+\left|\zeta_{0}\right|^{2}|z|^{2}+O\left(|z|^{3}\right)\right)-\frac{\alpha_{i}^{4} \gamma_{0}}{|z|^{4}}\right)|d z|^{2} \\
& =\frac{\alpha_{i}^{4}}{|z|^{6}}\left(v^{2}\left(p_{i}\right)+2 \operatorname{Re}\left(\zeta_{0} v\left(p_{i}\right) z\right)+\left(\left|\zeta_{0}\right|^{2}-\gamma_{0}\right)|z|^{2}+O\left(|z|^{3}\right)\right)
\end{aligned}
$$

Therefore, we obtain

$$
\begin{aligned}
|\partial u|_{g}^{2}= & e^{-2 \lambda}\left|\partial_{z} u\right|^{2}=\frac{|z|^{4}}{\alpha_{i}^{2}}\left(1+2 \operatorname{Re}\left(\alpha_{0} z^{2}\right)+O\left(|z|^{3}\right)\right) \\
& \times \frac{\alpha_{i}^{4}}{|z|^{6}}\left(v^{2}\left(p_{i}\right)+2 \operatorname{Re}\left(\zeta_{0} v\left(p_{i}\right) z\right)+\left(\left|\zeta_{0}\right|^{2}-\gamma_{0}\right)|z|^{2}+O\left(|z|^{3}\right)\right) \\
= & \frac{\alpha_{i}^{2}}{|z|^{2}}\left(v^{2}\left(p_{i}\right)+2 \operatorname{Re}\left(\zeta_{0} v\left(p_{i}\right) z+\alpha_{0} z^{2}\right)\right)+\left(\left|\zeta_{0}\right|^{2}-\gamma_{0}\right)+O(|z|)
\end{aligned}
$$

and notice the constant $\left(\left|\zeta_{0}\right|^{2}-\gamma_{0}\right)$. Finally, we find for some $\lambda_{2}, \lambda_{3} \in \mathbb{C}$

$$
\partial|\partial u|_{g}^{2}=-\frac{\alpha_{i}^{2}}{z|z|^{2}}\left(v^{2}\left(p_{i}\right)+\overline{\zeta_{0}} v\left(p_{i}\right) \bar{z}+\lambda_{2} z^{2}+\lambda_{3} \bar{z}^{2}\right) d z+O(1)
$$

Finally, we have

$$
\begin{aligned}
-\partial\left|d\left(|\vec{\Phi}|^{2} v\right)\right|_{g}^{2} & =-\partial|d u|_{g}^{2}=-4 \partial|\partial u|_{g}^{2} \\
& =\frac{4 \alpha_{i}^{2}}{z|z|^{2}}\left(v^{2}\left(p_{i}\right)+\overline{\zeta_{0}} v\left(p_{i}\right) \bar{z}+\lambda_{2} z^{2}+\lambda_{3} \bar{z}^{2}\right) d z+O(1) .
\end{aligned}
$$

and

$$
\operatorname{Im} \int_{\partial B(0, \varepsilon)}-\partial\left|d\left(|\vec{\Phi}|^{2} v\right)\right|_{g}^{2}=\frac{8 \pi \alpha_{i}^{2}}{\varepsilon^{2}}+O(\varepsilon) .
$$

Gathering (7.6) and (7.7) we obtain as $K_{g}=O\left(|z|^{6}\right)$ by planarity of the end (notice the factor 2 in front of the Laplacian)

$$
\begin{aligned}
\operatorname{Im} \int_{\partial B(0, \varepsilon)} 2\left(\Delta_{g} u+2 K_{g} u\right) \partial u-\partial|d u|_{g}^{2} & =\operatorname{Im} \int_{\partial B(0, \varepsilon)} 2\left(\Delta_{g} u\right) \partial u-\partial|d u|_{g}^{2}+O(\varepsilon) \\
& =2\left(-\frac{8 \pi \alpha_{i}^{2}}{\varepsilon^{2}}+4 \pi \alpha_{i}^{2} \gamma_{1} v\left(p_{i}\right)\right)+\frac{8 \pi \alpha_{i}^{2}}{\varepsilon^{2}}+O(\varepsilon) \\
& =-\frac{8 \pi \alpha_{i}^{2}}{\varepsilon^{2}}+8 \pi \alpha_{i}^{2} \gamma_{1} v\left(p_{i}\right)+O(\varepsilon)
\end{aligned}
$$


Now, coming back to (7.3), we see that for $v_{0}$ written above we have (with $\beta_{i}$ replace by $\alpha_{i}$ )

$$
\gamma_{1}=\sum_{j \neq i} \frac{\lambda_{i, j}}{\alpha_{i}^{2}} v\left(p_{j}\right)
$$

so that

$$
8 \pi \alpha_{i}^{2} \gamma_{1} v\left(p_{i}\right)=8 \pi \sum_{j \neq i} \lambda_{i, j} v\left(p_{i}\right) v\left(p_{j}\right) .
$$

Therefore, we get for all $1 \leq i \leq n$

$$
\begin{aligned}
& \int_{\partial B_{\varepsilon}\left(p_{i}\right)}\left(\Delta_{g} u_{0}+2 K_{g} u_{0}\right) * d u_{0}-\frac{1}{2} * d\left|d u_{0}\right|_{g}^{2} \\
& =-\frac{8 \pi \alpha_{i}^{2}}{\varepsilon^{2}} v^{2}\left(p_{i}\right)+8 \pi \sum_{\substack{j=1 \\
j \neq i}}^{n} \lambda_{i, j} v\left(p_{i}\right) v\left(p_{j}\right)+O\left(\varepsilon \log ^{2} \varepsilon\right),
\end{aligned}
$$

and

$$
\begin{aligned}
& \sum_{i=1}^{n} \int_{\partial B_{\varepsilon}\left(p_{i}\right)}\left(\Delta_{g} u_{0}+2 K_{g} u_{0}\right) * d u_{0}-\frac{1}{2} * d\left|d u_{0}\right|_{g}^{2} \\
& \quad=-8 \pi \sum_{i=1}^{n} \frac{\alpha_{i}^{2}}{\varepsilon^{2}} v^{2}\left(p_{i}\right)+8 \pi \sum_{\substack{i, j=1 \\
i \neq j}}^{n} \lambda_{i, j} v\left(p_{i}\right) v\left(p_{j}\right)+O\left(\varepsilon \log ^{2} \varepsilon\right) .
\end{aligned}
$$

However, we remark that for this new variation, the proof of Theorem 6.6 does not apply since we neglected terms involving $\partial_{\nu} v$, which could not remain in the limit for a $C^{2}$ variation (by Sobolev embedding). In particular, the formulas involving $u_{\varepsilon}^{i}$ do not apply in general. Therefore, we will use another argument, which is shorter. Since $u_{0}$ is a bi-Jacobi field $\mathscr{L}_{g}^{2} u_{0}=0$, we can simply integrate by parts to find

$$
\frac{1}{2} \int_{\Sigma_{\varepsilon}}\left(\mathscr{L}_{g} u_{0}\right)^{2} d \operatorname{vol}_{g}=\sum_{i=1}^{n} \frac{1}{2} \int_{\partial B_{\varepsilon}\left(p_{i}\right)} u_{0} \partial_{\nu}\left(\mathscr{L}_{g} u_{0}\right)-\partial_{\nu} u_{0} \mathscr{L}_{g} u_{0} d \mathscr{H}^{1} .
$$

Recall that

$$
\Delta_{g} u=4 v\left(p_{i}\right)+2 \operatorname{Re}\left(\zeta_{3} z^{2}\right)+O\left(|z|^{3}\right),
$$

which implies since $K_{g}=O\left(|z|^{6}\right)$ that

$$
\mathscr{L}_{g} u_{0}^{i}=4 v\left(p_{i}\right)+2 \operatorname{Re}\left(\zeta_{3} z^{2}\right)+O\left(|z|^{3}\right) .
$$

Therefore, by the formula (valid for all $\varphi \in C^{\infty}(B(0,1), \mathbb{R})$ )

$$
\int_{B_{\varepsilon}\left(p_{i}\right)} \partial_{\nu} \varphi d \mathscr{H}^{1}=2 \operatorname{Im} \int_{\partial B(0, \varepsilon)} \operatorname{Re}\left(z \cdot \partial_{z} \varphi\right) \frac{d z}{z}
$$

we have

$$
\int_{\partial B(0, \varepsilon)} u_{0}^{i} \partial_{\nu}\left(\mathscr{L}_{g} u_{0}^{i}\right)-\partial_{\nu} u_{0}^{i} \mathscr{L}_{g} u_{0}^{i} d \mathscr{H}^{1}
$$




$$
\begin{aligned}
= & 2 \operatorname{Im} \int_{B(0, \varepsilon)} \frac{\alpha_{i}^{2}}{|z|^{2}}\left(v\left(p_{i}\right)+2 \operatorname{Re}\left(\zeta_{0} z+\zeta_{2} z^{2}\right)\right) 2 \operatorname{Re}\left(\zeta_{3} z^{2}\right) \frac{d z}{z} \\
& -2 \operatorname{Im} \int_{\partial B(0, \varepsilon)}\left(-\frac{\alpha_{i}^{2}}{|z|^{2}}\left(v\left(p_{i}\right)-\operatorname{Re}\left(\zeta_{0} z\right)\right)+\frac{1}{2} \sum_{j=1, j \neq i}^{n} \lambda_{i, j} v\left(p_{j}\right)\right) \\
& \left(4 v\left(p_{i}\right)+2 \operatorname{Re}\left(\zeta_{3} z^{2}\right)\right)+O(\varepsilon \log \varepsilon) \\
= & \frac{16 \pi \alpha_{i}^{2}}{\varepsilon^{2}} v^{2}\left(p_{i}\right)-8 \pi \sum_{\substack{j=1 \\
j \neq i}}^{n} \lambda_{i, j} v\left(p_{i}\right) v\left(p_{j}\right)+O(\varepsilon \log \varepsilon)
\end{aligned}
$$

and finally

$$
\begin{aligned}
& \frac{1}{2} \int_{\partial B_{\varepsilon}\left(p_{i}\right)} u_{0} \partial_{\nu}\left(\mathscr{L}_{g} u_{0}\right)-\partial_{\nu} u_{0} \mathscr{L}_{g} u_{0} d \mathscr{H}^{1} \\
& \quad=\frac{8 \pi \alpha_{i}^{2}}{\varepsilon^{2}} v^{2}\left(p_{i}\right)-4 \pi \sum_{\substack{j=1 \\
j \neq i}}^{n} \lambda_{i, j} v\left(p_{i}\right) v\left(p_{j}\right)+O(\varepsilon \log \varepsilon) .
\end{aligned}
$$

Therefore, we have by (7.12) and (7.13)

$$
\begin{aligned}
\frac{1}{2} \int_{\Sigma_{\varepsilon}}\left(\mathscr{L}_{g} u_{0}\right)^{2} d \operatorname{vol}_{g} & =8 \pi \sum_{i=1}^{n} \frac{\alpha_{i}^{2}}{\varepsilon^{2}} v^{2}\left(p_{i}\right)-4 \pi \sum_{i=1}^{n} \sum_{\substack{j=1 \\
j \neq i}}^{n} \lambda_{i, j} v\left(p_{i}\right) v\left(p_{j}\right)+O(\varepsilon \log \varepsilon) \\
& =8 \pi \sum_{i=1}^{n} \frac{\alpha_{i}^{2}}{\varepsilon^{2}} v^{2}\left(p_{i}\right)-4 \pi \sum_{\substack{i, j=1 \\
i \neq j}}^{n} \lambda_{i, j} v\left(p_{i}\right) v\left(p_{j}\right)+O(\varepsilon \log \varepsilon)
\end{aligned}
$$

By [28], we have

$$
Q_{\vec{\Psi}}\left(v_{0}\right)=\lim _{\varepsilon \rightarrow 0}\left\{\frac{1}{2} \int_{\Sigma_{\varepsilon}}\left(\mathscr{L}_{g} u_{0}\right)^{2} d \operatorname{vol}_{g}+\sum_{i=1}^{n} \int_{\partial B_{\varepsilon}\left(p_{i}\right)}\left(\Delta_{g} u_{0}+2 K_{g} u_{0}\right) * d u_{0}-\frac{1}{2} * d\left|d u_{0}\right|_{g}^{2}\right\} .
$$

Finally, combining (7.11), (7.14) and (7.15), we deduce that

$$
Q_{\vec{\Psi}}\left(v_{0}\right)=4 \pi \sum_{1 \leq i, j \leq n} \lambda_{i, j} v\left(p_{i}\right) v\left(p_{j}\right),
$$

and this concludes the proof of the theorem (the proof for catenoid ends is almost identical, up to the additional coming from the flux which remains unchanged).

\section{Renormalised energy for ends of arbitrary multiplicity}

Theorem 8.1 Let $\vec{\Phi}: \Sigma \backslash\left\{p_{1}, \ldots, p_{n}\right\} \rightarrow \mathbb{R}^{3}$ be a complete minimal surface with finite total curvature and $\vec{\Psi}=\iota \circ \vec{\Phi}: \Sigma \rightarrow \mathbb{R}^{3}$ be a compact inversion of $\vec{\Phi}$. Then there a universal symmetric matrix $\Lambda=\Lambda(\vec{\Psi})=\left\{\lambda_{i, j}\right\}_{1 \leq i, j \leq n}$ with such that for smooth all 
smooth admissible variation $\vec{v} \in \operatorname{Var}(\vec{\Psi}) \cap C^{\infty}\left(\Sigma, \mathbb{R}^{3}\right)$, we have

$$
Q_{\vec{\Psi}}(\vec{v})=\frac{1}{2} \int_{\Sigma}\left(\Delta_{g} u_{0}-2 K_{g} u_{0}\right)^{2} d \operatorname{vol}_{g}+4 \pi \sum_{1 \leq i, j \leq n} \lambda_{i, j} v\left(p_{i}\right) v\left(p_{j}\right),
$$

where $u_{0}=|\vec{\Phi}|^{2} v_{0}$ for some for some $v_{0} \in W^{2,2}(\Sigma, \mathbb{R})$ such that $v_{0}\left(p_{i}\right)=0$ for all $1 \leq i \leq n$. In particular, we have

$$
\operatorname{Ind}_{W}(\vec{\Psi}) \leq \operatorname{Ind} \Lambda(\vec{\Psi}) \leq \frac{1}{4 \pi} W(\vec{\Psi})-\frac{1}{2 \pi} \int_{S^{2}} K_{g} d \operatorname{vol}_{g}+\chi(\Sigma) .
$$

Proof As previously, fix a some residue charts $\left(U_{1}, \ldots, U_{n}\right)$ around $p_{1}, \ldots, p_{n}$, and assume that $p_{i}$ has multiplicity $m_{i} \geq 1$ for all $1 \leq i \leq n$, and fix some $1 \leq i \leq m$. By the discussion before the proof of Theorem 5.1, we have

$$
Q_{\vec{\Psi}}(\vec{v})=\lim _{\varepsilon \rightarrow 0}\left\{\frac{1}{2} \int_{\Sigma_{\varepsilon}}\left(\mathscr{L}_{g} u\right)^{2} d \operatorname{vol}_{g}-\sum_{i=1}^{n} Q_{\varepsilon}^{i}(v)\right\}+\sum_{i=1}^{n} \gamma_{0, i}(v) v\left(p_{i}\right)
$$

where $Q_{\varepsilon}^{i}$ is defined by (5.6), and $\gamma_{0, i}(v)$ only depends on the germ of $v$ at $p_{i}$. Now, applying the proof of Theorem 6.6, since the previous limit is finished, we must have

$$
Q_{\varepsilon}\left(u_{\varepsilon}^{i}\right)=\frac{1}{2} \int_{\Sigma_{\varepsilon}}\left(\mathscr{L}_{g} u_{\varepsilon}^{i}\right)^{2} d \operatorname{vol}_{g}=Q_{\varepsilon}^{i}(v)+\gamma_{i}(v)+O\left(\varepsilon \log ^{N} \varepsilon\right) .
$$

where $\gamma_{i}(v)$ only depends on the germ of $v$ at $p_{i}$.

For the sake of simplicity, we will remove the indices $i$ of the multiplicities $m_{i}(1 \leq i \leq n)$.

First notice that for all $k \neq i, j$, we have if $p_{k}$ has multiplicity $m_{k}=m \geq 2$ (for $m=1$ this was already treated previously)

$$
\begin{aligned}
& u_{\varepsilon}^{i}=\operatorname{Re}\left(\frac{\gamma_{i, k}}{z^{m}}\right)+O\left(|z|^{1-m}\right) \\
& u_{\varepsilon}^{j}=\operatorname{Re}\left(\frac{\gamma_{j, k}}{z^{m}}\right)+O\left(|z|^{1-m}\right) . \\
& \partial_{\nu} u_{\varepsilon}^{i}=O\left(|z|^{-(m+1)}\right) \\
& \partial_{\nu} u_{\varepsilon}^{j}=O\left(|z|^{-(m+1)}\right)
\end{aligned}
$$

As $e^{\lambda}=\alpha_{k}^{2}|z|^{-2(m+1)}(1+O(|z|))$, and $K_{g}=O\left(|z|^{2(m+1)}\right)$. Therefore, we have by the harmonicity of $\operatorname{Re}\left(c z^{-m}\right)$ for all $c \in \mathbb{C}$

$$
\begin{aligned}
& \Delta u_{\varepsilon}^{i}=O\left(|z|^{-(m+1)}\right) \\
& \Delta u_{\varepsilon}^{j}=O\left(|z|^{-(m+1)}\right),
\end{aligned}
$$

so that (as $\Delta_{g}=e^{-2 \lambda} \Delta$ )

$$
\begin{aligned}
& \mathscr{L}_{g} u_{\varepsilon}^{i}=\Delta_{g} u_{\varepsilon}^{i}-2 K_{g} u_{\varepsilon}^{i}=O\left(|z|^{m+1}\right) \\
& \mathscr{L}_{g} u_{\varepsilon}^{j}=O\left(|z|^{m+1}\right) .
\end{aligned}
$$

Therefore, we have

$$
\begin{aligned}
& u_{\varepsilon}^{i} \partial_{\nu}\left(\mathscr{L}_{g} u_{\varepsilon}^{j}\right)=O\left(|z|^{-m}\right) \times O\left(|z|^{m}\right)=O(1) \\
& \partial_{\nu}\left(u_{\varepsilon}^{i}\right) \mathscr{L}_{g} u_{\varepsilon}^{j}=O\left(|z|^{-(m+1)}\right) \times O\left(|z|^{m+1}\right)=O(1) .
\end{aligned}
$$


This implies that

$$
u_{\varepsilon}^{i} \partial_{\nu}\left(\mathscr{L}_{g} u_{\varepsilon}^{j}\right)-\partial_{\nu}\left(u_{\varepsilon}^{i}\right) \mathscr{L}_{g} u_{\varepsilon}^{j}=O(1)
$$

and

$$
\int_{\partial B_{\varepsilon}\left(p_{k}\right)} u_{\varepsilon}^{i} \partial_{\nu}\left(\mathscr{L}_{g} u_{\varepsilon}^{j}\right)-\partial_{\nu}\left(u_{\varepsilon}^{i}\right) \mathscr{L}_{g} u_{\varepsilon}^{j} d \mathscr{H}^{1}=O(\varepsilon) .
$$

As the indices $i$ and $j$ do not play any role, we also have

$$
\int_{\partial B_{\varepsilon}\left(p_{k}\right)} u_{\varepsilon}^{j} \partial_{\nu}\left(\mathscr{L}_{g} u_{\varepsilon}^{i}\right)-\partial_{\nu}\left(u_{\varepsilon}^{j}\right) \mathscr{L}_{g} u_{\varepsilon}^{i} d \mathscr{H}^{1}=O(\varepsilon) .
$$

Now, by (8.1) and (5.6), we deduce that in a neighourhood of $p_{i}$, we have

$$
\mathscr{L}_{g} u_{\varepsilon}^{i}=\mathscr{L}_{g} u+O\left(|z|^{m+1}\right)=f_{0} v\left(p_{i}\right)+O\left(|z|^{m+1}\right)=(4+O(|z|)) v\left(p_{i}\right)+O\left(|z|^{m+1}\right)
$$

Therefore, we have

$$
\begin{aligned}
\partial_{\nu} & u_{\varepsilon}^{j} \mathscr{L}_{g} u_{\varepsilon}^{i}-u_{\varepsilon}^{i} \partial_{\nu}\left(\mathscr{L}_{g} u_{\varepsilon}^{i}\right) \\
= & \frac{1}{|z|}\left(-m \operatorname{Re}\left(\frac{c_{i, j}}{z^{m}}\right)+\sum_{1-m \leq k+l \leq 0} \operatorname{Re}\left(k c_{k, l}^{j} z^{k} \bar{z}^{l}\right)+\gamma_{i, j}\right) f_{0} v\left(p_{i}\right) \\
& +\left(\operatorname{Re}\left(\frac{c_{i, j}}{z^{m}}\right)+\sum_{1-m \leq k+l \leq 0} \operatorname{Re}\left(c_{k, l}^{j} z^{k} \bar{z}^{l}\right)+\gamma_{i, j} \log |z|\right) \partial_{\nu} f_{0} v\left(p_{i}\right) .
\end{aligned}
$$

Notice that the quantity

$$
B_{\varepsilon}\left(u_{\varepsilon}^{i}, u_{\varepsilon}^{j}\right)
$$

is bounded as $\varepsilon \rightarrow 0$. Therefore, cancellations occurs as we integrate (8.2). Furthermore, there is a non-trivial contribution coming from (as $K_{g}|\vec{\Phi}|^{2}=O\left(|z|^{2}\right)$ )

$$
\int_{\partial B_{\varepsilon}\left(p_{i}\right)} \frac{\gamma_{i, j}}{|z|}\left(4-K_{g}|\vec{\Phi}|^{2}\right) v\left(p_{i}\right) d \mathscr{H}^{1}=8 \pi \gamma_{i, j} v\left(p_{i}\right)+O\left(\varepsilon^{2}\right) .
$$

As $B_{\varepsilon}\left(u_{\varepsilon}^{i}, u_{\varepsilon}^{j}\right)$ is bounded, we deduce that there exists $\mu_{i, j} \in \mathbb{R}$ (a priori different from $\gamma_{i, j}$ if the multiplicity $m$ satisfies $m \geq 2$ ) such that

$$
\int_{\partial B_{\varepsilon}\left(p_{i}\right)} \partial_{\nu} u_{\varepsilon}^{j} \mathscr{L}_{g} u_{\varepsilon}^{i}-u_{\varepsilon}^{i} \partial_{\nu}\left(\mathscr{L}_{g} u_{\varepsilon}^{i}\right) d \mathscr{H}^{1}=8 \pi \mu_{i, j} v\left(p_{i}\right)+O(\varepsilon) .
$$

Finally, recall if $u_{\varepsilon}^{i}=e^{\lambda} w_{\varepsilon}^{i}$ that in our fixed chart near $p_{j}$ (if $p_{j}$ has multiplicity $m_{j}=m \geq 2$ )

$$
\begin{aligned}
w_{\varepsilon}^{i}(z)= & |z|^{m+1} \sum_{k=1}^{m} \operatorname{Re}\left(\frac{\gamma_{0}^{k}}{z^{k}}\right)+|z|^{1-m} \sum_{k=0}^{m-1} \operatorname{Re}\left(\gamma_{1}^{j} z^{m+k}\right) \\
& +\gamma_{2}|z|^{m+1}+\gamma_{3}|z|^{m+1} \log |z|+O\left(|z|^{m+2}\right) .
\end{aligned}
$$

Recalling that

$$
\mathscr{L}_{m}=\Delta-2(m+1) \frac{x}{|x|^{2}} \cdot \nabla+\frac{(m+1)^{2}}{|z|^{2}}
$$


and introducing the notation

$$
\mathscr{L}_{g} u_{\varepsilon}^{i}=e^{-\lambda} \mathscr{L} w_{\varepsilon}^{i}
$$

Recalling that for all $k \geq 1$

$$
|z|^{m+1} \operatorname{Re}\left(\frac{\gamma_{0}^{j}}{z^{k}}\right),|z|^{1-m} \operatorname{Re}\left(\gamma_{1}^{0} z^{m}\right),|z|^{m+1},|z|^{m+1} \log |z| \in \operatorname{Ker}\left(\mathscr{L}_{m}\right),
$$

we deduce that

$$
\mathscr{L}_{m} w_{\varepsilon}^{i}=|z|^{-(m+1)} \sum_{j=1}^{m-1} \operatorname{Re}\left(\widetilde{\gamma}_{1}^{k} z^{m+j}\right)+O\left(|z|^{m}\right) .
$$

Now, recall that $\vec{\Phi}$ admits an expansion of the following form (up to translation)

$$
\vec{\Phi}(z)=\sum_{k=0}^{m-1} \operatorname{Re}\left(\frac{\vec{A}_{j}}{z^{m-k}}\right)+O(|z|) .
$$

Therefore, we have (as $\left\langle\vec{A}_{0}, \vec{A}_{1}\right\rangle=0$ )

$$
\begin{aligned}
& |\vec{\Phi}(z)|^{2}=\frac{1}{2} \sum_{k=0}^{m-1} \frac{\left|\vec{A}_{k}\right|^{2}}{|z|^{2(m-k)}}+\frac{1}{2} \sum_{0 \leq k<l \leq m-1} \operatorname{Re}\left(\frac{\left\langle\overline{\vec{A}_{k}}, \vec{A}_{l}\right\rangle}{\bar{z}^{m-k} \bar{z}^{m-l}}\right) \\
& +\frac{1}{2} \sum_{\substack{0 \leq k<l \\
(k, l) \neq(0,1)}} \operatorname{Re}\left(\frac{\left\langle\vec{A}_{k}, \vec{A}_{l}\right\rangle}{z^{2 m-k-l}}\right)+O\left(|z|^{1-m}\right) .
\end{aligned}
$$

As $e^{2 \lambda}=\partial_{z \bar{z}}^{2}|\vec{\Phi}|^{2}$, we find

$$
\begin{aligned}
e^{2 \lambda}= & \frac{1}{2} \sum_{k=0}^{m-1} \frac{(m-k)^{2}\left|\vec{A}_{k}\right|^{2}}{|z|^{2(m+1-k)}}+\frac{1}{2} \sum_{0 \leq k<l \leq m-1}(m-k)(m-l) \operatorname{Re}\left(\frac{\left\langle\overrightarrow{\vec{A}}_{k}, \vec{A}_{l}\right\rangle}{\bar{z}^{m+1-k} z^{m+1-l}}\right) \\
& +O\left(|z|^{-(m+1)}\right) \\
= & \frac{m^{2}\left|\vec{A}_{0}\right|^{2}}{2|z|^{2(m+1)}}\left(1+\sum_{k=1}^{m-1}\left(1-\frac{k}{m}\right) \frac{\left|\vec{A}_{k}\right|^{2}}{\left|\vec{A}_{0}\right|^{2}}|z|^{2 k}\right. \\
& \left.+\sum_{0 \leq k<l \leq m-1}\left(1-\frac{k}{m}\right)\left(1-\frac{l}{m}\right) \operatorname{Re}\left(\frac{\left\langle\vec{A}_{k}, \vec{A}_{l}\right\rangle}{\left|\vec{A}_{0}\right|^{2}} z^{l} \bar{z}^{k}\right)+O\left(|z|^{m+1}\right)\right)
\end{aligned}
$$

Therefore, we have (up to normalisation $m^{2}\left|\vec{A}_{0}\right|^{2}=2$ ) for some $\alpha_{k, l} \in \mathbb{C}$ and $\beta_{k} \in \mathbb{R}$

$$
e^{-\lambda}=|z|^{m+1}\left(1+\sum_{k=1}^{(m-1) / 2} \beta_{k}|z|^{2 k}+\sum_{\substack{0 \leq k<l \leq m-1 \\ k+l \leq m}} \operatorname{Re}\left(\alpha_{k, l} z^{k} \bar{z}^{l}\right)+O\left(|z|^{m+1}\right)\right),
$$

and

$$
\mathscr{L}_{g} u_{\varepsilon}^{i}=\left(1+\sum_{k=1}^{(m-1) / 2} \beta_{k}|z|^{2 k}+\sum_{0 \leq k<l \leq m-1} \operatorname{Re}\left(\alpha_{k, l} z^{k} \bar{z}^{l}\right)+O\left(|z|^{m+1}\right)\right)
$$




$$
\begin{aligned}
& \times\left(\sum_{k=1}^{m-1} \operatorname{Re}\left(\tilde{\gamma}_{1}^{k} z^{m+k}\right)+O\left(|z|^{2 m+1}\right)\right) \\
= & \operatorname{Re}\left(\tilde{\gamma}_{1}^{1} z^{m+1}\right)+\sum_{\substack{0 \leq k<l \leq m-1 \\
m+2 \leq k+l}} \operatorname{Re}\left(\widetilde{\alpha}_{k, l} z^{k} \bar{z}^{l}\right)+O\left(|z|^{2 m+1}\right) .
\end{aligned}
$$

Furthermore, we have on $\partial B_{\varepsilon}\left(p_{j}\right)$ thanks to Theorem 4.1

$$
u_{\varepsilon}^{j}=f_{1} v\left(p_{i}\right)+O\left(|z|^{-m}\right),
$$

where $f_{1}=|\vec{\Phi}|^{2}(1+O(|z|))$. Therefore, we deduce that

$$
u_{\varepsilon}^{j} \partial_{\nu}\left(\mathscr{L}_{g} u_{\varepsilon}^{i}\right)-\partial_{\nu}\left(u_{\varepsilon}^{j}\right) \mathscr{L}_{g} u_{\varepsilon}^{i}=\left(f_{1} \partial_{\nu}\left(\mathscr{L}_{g} u_{\varepsilon}^{i}\right)-\partial_{\nu}\left(f_{1}\right) \mathscr{L}_{g} u_{\varepsilon}^{i}\right) v\left(p_{j}\right)+O(1)
$$

As $B_{\varepsilon}\left(u_{\varepsilon}^{i}, u_{\varepsilon}^{j}\right)$ is bounded, we deduce as previously that there exists $v_{i, j} \in \mathbb{R}$ such that

$$
\int_{\partial B_{\varepsilon}\left(p_{j}\right)} u_{\varepsilon}^{j} \partial_{\nu}\left(\mathscr{L}_{g} u_{\varepsilon}^{i}\right)-\partial_{\nu}\left(u_{\varepsilon}^{j}\right) \mathscr{L}_{g} u_{\varepsilon}^{i} d \mathscr{H}^{1}=8 \pi v_{i, j} v\left(p_{j}\right)+O(\varepsilon) .
$$

Therefore, we deduce that (as we may have also ends of multiplicity 1, there is an additional error in $O(\varepsilon \log \varepsilon))$

$$
B_{\varepsilon}\left(u_{\varepsilon}^{i}, u_{\varepsilon}^{j}\right)=8 \pi \mu_{i, j} v\left(p_{i}\right)+8 \pi v_{i, j} v\left(p_{j}\right)+O(\varepsilon \log \varepsilon) .
$$

Now assume that $v\left(p_{j}\right)=0$ and $v\left(p_{i}\right) \neq 0$. Then

$$
B_{\varepsilon}\left(u_{\varepsilon}^{i}, u_{\varepsilon}^{j}\right)=8 \pi \mu_{i, j} v\left(p_{i}\right)+O(\varepsilon \log \varepsilon)
$$

and by symmetry, in $i$ and $j$, we deduce that

$$
B_{\varepsilon}\left(u_{\varepsilon}^{i}, u_{\varepsilon}^{j}\right)=8 \pi \mu_{j, i} v\left(p_{j}\right)+O(\varepsilon \log \varepsilon)=0 .
$$

Therefore, $v\left(p_{j}\right)=0$ implies that $\mu_{i, j}=0$, which shows that there exists $\lambda_{i, j}^{1} \in \mathbb{R}$ such that

$$
\mu_{i, j}=\lambda_{i, j}^{1} v\left(p_{j}\right)
$$

Furthermore, by symmetry of the argument, we deduce that there exists $\lambda_{i, j}^{2} \in \mathbb{R}$ such that

$$
v_{i, j}=\lambda_{i, j}^{2} v\left(p_{i}\right)
$$

Therefore, if $\lambda_{i, j}^{3}=\lambda_{i, j}^{1}+\lambda_{i, j}^{2}$, we deduce that

$$
B_{\varepsilon}\left(u_{\varepsilon}^{i}, u_{\varepsilon}^{j}\right)=8 \pi \lambda_{i, j}^{3} v\left(p_{i}\right) v\left(p_{j}\right)+O(\varepsilon \log \varepsilon) .
$$

Likewise, we find by the previous argument and the proof of Theorem 6.6 that there exists $\lambda_{i, j}^{4} \in \mathbb{R}$ such that

$$
B_{\varepsilon}\left(u_{\varepsilon}, u_{\varepsilon}^{i}\right)=4 \pi \sum_{j \neq i} \lambda_{i, j}^{4} v\left(p_{i}\right) v\left(p_{j}\right)+O(\varepsilon \log \varepsilon) .
$$

Combining (8.5) and (8.6), we deduce if $\lambda_{i, j}=\lambda_{i, j}^{3}+\lambda_{i, j}^{4}$ that

$$
\sum_{i=1}^{n} B_{\varepsilon}\left(u_{\varepsilon}, u_{\varepsilon}^{i}\right)+\frac{1}{2} \sum_{1 \leq i \neq j \leq n} B_{\varepsilon}\left(u_{\varepsilon}^{i}, u_{\varepsilon}^{j}\right)=4 \pi \sum_{1 \leq i \neq j \leq n} \lambda_{i, j} v\left(p_{i}\right) v\left(p_{j}\right)+O\left(\varepsilon \log ^{2} \varepsilon\right) .
$$


Therefore, (8.1) and (8.7) show that

$$
Q_{\varepsilon}(u)=Q_{\varepsilon}\left(u_{\varepsilon}\right)+4 \pi \sum_{\substack{1 \leq i, j \leq n \\ i \neq j}} \lambda_{i, j} v\left(p_{i}\right) v\left(p_{j}\right)+O\left(\varepsilon \log ^{2} \varepsilon\right)
$$

and finally

$$
\begin{aligned}
Q_{\vec{\Psi}}(\vec{v})= & \frac{1}{2} \int_{\Sigma}\left(\mathscr{L}_{g} u_{0}-2 K_{g} u_{0}\right)^{2} d \operatorname{vol}_{g}+4 \pi \sum_{\substack{1 \leq i, j \leq n \\
i \neq j}} \lambda_{i, j} v\left(p_{i}\right) v\left(p_{j}\right) \\
& +\sum_{i=1}^{n} \gamma_{0, i}(v) v\left(p_{i}\right)+\sum_{i=1}^{n} \gamma_{i}(v),
\end{aligned}
$$

where $\gamma_{0, i}$ is a linear function of $v$ that only depends on the germ of $v$ at $p_{i}$, and $\gamma_{i}(v)$ is a quadratic function of $v$ that only depends on the germ on $v$ at $p_{i}$. Since the second derivative $Q_{\vec{\Psi}}$ is continuous with respect to the admissible variation $\vec{v} \in \operatorname{Var}(\vec{\Psi})$ (see the explicit expression in [28]), we deduce that

$$
\gamma_{0, i}(v) v\left(p_{i}\right)+\gamma_{i}(v)=4 \pi \lambda_{i, j} v^{2}\left(p_{i}\right)
$$

for some $\lambda_{i, i} \in \mathbb{R}$ depending only on $\vec{\Phi}$. This concludes the proof of the theorem.

We deduce as previously the following corollary.

Corollary 8.2 For all $1 \leq i \leq n$, there exists $\tilde{\lambda}_{i, j} \in \mathbb{R}$ such that for all $j \neq i$, for all $0<\varepsilon<\varepsilon_{0}$, on every complex chart around $p_{j}$ there exists $c_{i, j}, c_{i, j, k, l} \in \mathbb{C}$

$$
u_{\varepsilon}^{i}(z)=\operatorname{Re}\left(\frac{c_{i, j}}{z^{m}}\right)+\sum_{1-m \leq k+l \leq 0} \operatorname{Re}\left(c_{i, j, k, l} z^{k} \bar{z}^{l}\right)+\tilde{\lambda}_{i, j} \log |z|+\psi_{\varepsilon}(z),
$$

where $\psi_{\varepsilon} \in C^{\infty}(B(0,1) \backslash\{0\})$ such that for all $l \in \mathbb{N}$

$$
\nabla^{l} \psi_{\varepsilon}=O\left(|z|^{1-l}\right) .
$$

For ends of higher multiplicity $m \geq 2$, we do not know a priori if $\tilde{\lambda}_{i, j}=\lambda_{i, j}$, where $\lambda_{i, j} \in \mathbb{R}$ is given by Theorem. Nevertheless, the proof of Theorem 7.1 implies the following result.

Theorem 8.3 Let $\vec{\Phi}: \Sigma \backslash\left\{p_{1}, \ldots, p_{n}\right\} \rightarrow \mathbb{R}^{3}$ be a complete minimal surface with finite total curvature, and $\vec{\Psi}=\iota \circ \vec{\Phi}: \Sigma \rightarrow \mathbb{R}^{3}$ be a compact inversion of $\vec{\Phi}$, and let $\Lambda(\vec{\Psi})=$ $\left\{\lambda_{i, j}\right\}_{1 \leq i, j \leq n} \in \operatorname{Sym}_{n}(\mathbb{R})$ be the matrix given by Theorem 8.1 . Then we have

$$
\operatorname{Ind}_{W}(\vec{\Psi})=\operatorname{Ind} \Lambda(\vec{\Psi}) .
$$

Proof As in the proof of Theorem 7.1, we use the previous formula with $v_{0}=|\vec{\Phi}|^{-2} u_{0}$, which satisfies $v_{0}\left(p_{i}\right)=v\left(p_{i}\right)$ for all $1 \leq i \leq n$. If $\vec{v}_{0}=-v_{0} \vec{n}_{\vec{\Psi}}+2 \operatorname{Re}(\alpha \otimes \partial \vec{\Psi})$, where $\alpha$ is the same $(-1,0)$-form as the one associated to $v$, we compute

$$
Q_{\vec{\Psi}}\left(\vec{v}_{0}\right)=\lim _{\varepsilon \rightarrow}\left\{\frac{1}{2} \int_{\Sigma_{\varepsilon}}\left(\mathscr{L}_{g} u_{0}\right)^{2} d \operatorname{vol}_{g}+\sum_{i=1}^{n} \int_{\partial B_{\varepsilon}\left(p_{i}\right)} \omega\left(u_{0}, \alpha\right)\right\} .
$$


Since $u_{0}$ is biharmonic, we have

$$
\begin{aligned}
\frac{1}{2} \int_{\Sigma_{\varepsilon}}\left(\mathscr{L}_{g} u_{0}\right)^{2} d \operatorname{vol}_{g} & =\sum_{i=1}^{n} \frac{1}{2} \int_{\partial B_{\varepsilon}\left(p_{i}\right)} u_{0} \partial_{\nu}\left(\mathscr{L}_{g} u_{0}\right)-\partial_{\nu}\left(u_{0}\right) \mathscr{L}_{g} u_{0} d \mathscr{H}^{1} \\
& =\sum_{i=1}^{n} Q_{\varepsilon}^{i}(v)+O(1) .
\end{aligned}
$$

Now notice if $u_{0}=\sum_{i=1}^{n} u_{0}^{i}$ admits the expansion that

$$
u_{0}^{i}=|\vec{\Phi}|^{2} v\left(p_{i}\right)+\cdots+\sum_{j \neq i} \tilde{\lambda}_{i, j}^{0} v\left(p_{j}\right) \log |z|+O(1) .
$$

Therefore, we will get an extra term

$$
8 \pi \sum_{\substack{1 \leq i, j \leq n \\ i \neq j}}^{n} \tilde{\lambda}_{i, j}^{1} v\left(p_{i}\right) v\left(p_{j}\right)
$$

from (8.8) and another one

$$
8 \pi \sum_{\substack{1 \leq i, j \leq n \\ i \neq j}}^{n} \tilde{\lambda}_{i, j}^{2} v\left(p_{i}\right) v\left(p_{j}\right)
$$

from

$$
\sum_{i=1}^{n} \omega\left(u_{0}, \alpha\right)
$$

Therefore, we deduce that

$$
\begin{aligned}
Q_{\vec{\Psi}}\left(\vec{v}_{0}\right)= & 4 \pi \sum_{i=1}^{n} \lambda_{i, i} v^{2}\left(p_{i}\right)+4 \pi \sum_{\substack{1 \leq i, j \leq n \\
i \neq j}} \lambda_{i, j} v\left(p_{i}\right) v\left(p_{j}\right) \\
& +8 \pi \sum_{\substack{1 \leq i, j \leq n \\
i \neq j}} \tilde{\lambda}_{i, j}^{1} v\left(p_{i}\right) v\left(p_{j}\right)+8 \pi \sum_{\substack{1 \leq i, j \leq n \\
i \neq j}} \tilde{\lambda}_{i, j}^{2} v\left(p_{i}\right) v\left(p_{j}\right) \\
= & 4 \pi \sum_{i=1}^{n} \lambda_{i, i} v^{2}\left(p_{i}\right)+4 \pi \sum_{\substack{1 \leq i, j \leq n \\
i \neq j}} \tilde{\lambda}_{i, j} v\left(p_{i}\right) v\left(p_{j}\right)
\end{aligned}
$$

Now, let $\bar{\Lambda}(\vec{\Psi}) \in \operatorname{Sym}_{n}(\mathbb{R})$ the matrix

$$
\bar{\Lambda}(\vec{\Psi})=\left(\begin{array}{cccc}
0 & \tilde{\lambda}_{1,2}-\lambda_{1,2} & \ldots & \tilde{\lambda}_{1, n}-\lambda_{1, n} \\
\tilde{\lambda}_{1,2}-\lambda_{1,2} & 0 & \ldots & \tilde{\lambda}_{2, n}-\lambda_{2, n} \\
\vdots & \vdots & \ddots & \vdots \\
\tilde{\lambda}_{1, n}-\lambda_{1, n} & \tilde{\lambda}_{2, n}-\lambda_{2, n} & \ldots & 0
\end{array}\right) .
$$

By a standard approximation argument (see Theorem 5.1), there exists a sequence of smooth admissible variations $\left\{\vec{v}_{k}\right\}_{k \in \mathbb{N}} \subset \operatorname{Var}(\vec{\Psi}) \cap C^{\infty}\left(\Sigma, \mathbb{R}^{3}\right)$ such that

$$
\vec{v}_{k} \underset{k \rightarrow \infty}{\longrightarrow} \vec{v}_{0} \quad \text { in } \mathrm{W}^{2,2}\left(\Sigma, \mathbb{R}^{3}\right) \text {. }
$$


In particular, by continuity of the second derivative, we have

$$
Q_{\vec{\Psi}}\left(\vec{v}_{k}\right) \underset{k \rightarrow \infty}{\longrightarrow} Q_{\vec{\Psi}}\left(\vec{v}_{0}\right)=4 \pi \sum_{i=1}^{n} \lambda_{i, i} v^{2}\left(p_{i}\right)+4 \pi \sum_{\substack{1 \leq i, j \leq n \\ i \neq j}} \tilde{\lambda}_{i, j} v\left(p_{i}\right) v\left(p_{j}\right) .
$$

But since $\vec{v}_{k}$ is smooth, we have for all $k \in \mathbb{N}$ by Theorem 8.1

$$
Q_{\vec{\Psi}}\left(\vec{v}_{k}\right)=\frac{1}{2} \int_{\Sigma}\left(\mathscr{L}_{g} u_{k, 0}\right)^{2} d \operatorname{vol}_{g}+4 \pi \sum_{i, j=1}^{n} \lambda_{i, j} v\left(p_{i}\right) v\left(p_{j}\right) .
$$

Therefore, by (8.11), (8.12) and (8.13), we deduce that

$$
\frac{1}{2} \int_{\Sigma}\left(\mathscr{L}_{g} u_{k, 0}\right)^{2} d \operatorname{vol}_{g} \underset{k \rightarrow \infty}{\longrightarrow} 4 \pi \sum_{\substack{i, j=1 \\ i \neq j}}^{n} \bar{\lambda}_{i, j} v\left(p_{i}\right) v\left(p_{j}\right) .
$$

If $\bar{\Lambda}(\vec{\Psi})=0$, there is nothing to prove. Otherwise, since $\operatorname{Tr}(\vec{\Lambda} \vec{\Psi})=0$ (the trace of $\bar{\Lambda}(\vec{\Psi})$ is $0), \vec{\Psi}$ admits a negative eigenvalue, so there exists $v$ such that by (8.14)

$$
0 \leq \frac{1}{2} \int_{\Sigma}\left(\mathscr{L}_{g} u_{k, 0}\right)^{2} d \operatorname{vol}_{g} \underset{k \rightarrow \infty}{\longrightarrow} 4 \pi \sum_{\substack{i, j=1 \\ i \neq j}}^{n} \bar{\lambda}_{i, j} v\left(p_{i}\right) v\left(p_{j}\right)<0,
$$

contradicting the non-negativity of the right-hand side for $k$ large enough. Therefore, we deduce that $\bar{\Lambda}(\vec{\Psi})=0$, i.e. $\tilde{\lambda}_{i, j}=\lambda_{i, j}$ for all $1 \leq i, j \leq n$, which concludes the proof of the theorem.

\section{Morse index estimate for Willmore spheres in $S^{4}$}

Recall that we have from [26] we have the formula (valid in $\mathscr{D}^{\prime}(\Sigma)$ ) for all weak immersion $\vec{\Phi} \in \mathscr{E}\left(\Sigma, \mathbb{R}^{m}\right)$ and for all normal variations (see also the proof of Theorem 10.5 for a general formula valid for all variations)

$$
\begin{aligned}
\frac{d^{2}}{d t^{2}}\left(K_{g_{t}} d \operatorname{vol}_{g_{t}}\right)_{\mid t=0}= & d \operatorname{Im}\left(2\left\langle\Delta_{g}^{\perp} \vec{w}+4 \operatorname{Re}\left(g^{-2} \otimes(\bar{\partial} \vec{\Phi} \dot{\otimes} \vec{\partial}) \otimes \vec{h}_{0}\right), \partial \vec{w}\right\rangle\right. \\
& -\partial\left(\left|\nabla^{\perp} \vec{w}\right|_{g}^{2}\right) \\
& -2\langle d \vec{\Phi}, d \vec{w}\rangle_{g}\left(\langle\vec{H}, \partial \vec{w}\rangle-g^{-1} \otimes\left(\vec{h}_{0} \dot{\otimes} \bar{\partial} \vec{w}\right)\right) \\
& \left.-8 g^{-1} \otimes(\partial \vec{\Phi} \dot{\otimes} \partial \vec{w}) \otimes\langle\vec{H}, \bar{\partial} \vec{w}\rangle\right)
\end{aligned}
$$

In particular, as $\langle d \vec{\Phi}, d \vec{w}\rangle_{g}=-2\langle\vec{H}, \vec{w}\rangle$, for a minimal surface $(\vec{H}=0)$, we obtain

$$
\begin{aligned}
& \frac{d^{2}}{d t^{2}}\left(K_{g_{t}} d \operatorname{vol}_{g_{t}}\right)_{\mid t=0} \\
& \quad=d \operatorname{Im}\left(2\left\langle\Delta_{g}^{\perp} \vec{w}+4 \operatorname{Re}\left(g^{-2} \otimes(\bar{\partial} \vec{\Phi} \dot{\otimes} \bar{\partial} \vec{w}) \otimes \vec{h}_{0}\right), \partial \vec{w}\right\rangle-\partial\left(\left|\nabla^{\perp} \vec{w}\right|_{g}^{2}\right)\right) \\
& \quad=d \operatorname{Im}\left(2\left\langle\Delta_{g}^{\perp} \vec{w}-2 \operatorname{Re}\left(g^{-2} \otimes\left\langle\vec{w}, \vec{h}_{0}\right\rangle \otimes \vec{h}_{0}\right), \partial \vec{w}\right\rangle-\partial\left(\left|\nabla^{\perp} \vec{w}\right|_{g}^{2}\right)\right)
\end{aligned}
$$




$$
\begin{aligned}
& =d \operatorname{Im}\left(2\left\langle\Delta_{g}^{\perp} \vec{w}-\mathscr{A}(\vec{w}), \partial \vec{w}\right\rangle-\partial\left(\left|\nabla^{\perp} \vec{w}\right|_{g}^{2}\right)\right) \\
& =d\left(\left\langle\Delta_{g}^{\perp} \vec{w}-\mathscr{A}(\vec{w}), * d \vec{w}\right\rangle-\frac{1}{2} * d\left(\left|\nabla^{\perp} \vec{w}\right|_{g}^{2}\right)\right),
\end{aligned}
$$

where $\mathscr{A}(\vec{w})$ is the Simon's operator. Observe that the sign is different from the Jacobi operator $\mathscr{L}_{g}$ of the associated minimal surface, acting on normal sections of the pull-back bundle $\vec{\Phi}^{*} T \mathbb{R}^{m}$ as

$$
\mathscr{L}_{g}=\Delta_{g}^{\perp}+\mathscr{A}(\vec{w}) .
$$

Specialising further to the codimension 1 case $m=3$, as the minimal immersion that we consider is orientable, it is also two-sided and the unit normal furnishes a global trivialisation of the normal bundle so $\vec{w}=w \vec{n}$ for some $w \in W^{2,2}\left(S^{2}\right)$ and we get

$$
\begin{aligned}
\frac{d^{2}}{d t^{2}}\left(K_{g_{t}} d \operatorname{vol}_{g_{t}}\right)_{\mid t=0} & =d \operatorname{Im}\left(2\left(\Delta_{g} w+2 K_{g} w\right) \partial w-\partial\left(|d w|_{g}^{2}\right)\right) \\
& =d\left(\left(\Delta_{g} w+2 K_{g} w\right) * d w-\frac{1}{2} * d|d w|_{g}^{2}\right),
\end{aligned}
$$

and we recover the computation of [28]. We have the following generalisation of the aforecited result to $S^{4}$.

Theorem 9.1 Let $\vec{\Psi}: S^{2} \rightarrow S^{4}$ be a Willmore sphere, and $n \in \mathbb{N}$ such that $W(\vec{\Psi})=4 \pi n$ and assume that $\vec{\Phi}$ is conformally minimal in $\mathbb{R}^{4}$. Then we have $\operatorname{Ind}_{W}(\vec{\Psi}) \leq 2 n$.

Proof First, use some stereographic projection avoiding $\vec{\Psi}\left(S^{2}\right) \subset S^{4}$ to assume that $\vec{\Psi}$ : $S^{2} \rightarrow \mathbb{R}^{4}$ is a Willmore sphere. By Montiel's classification, let $\vec{\Phi}: S^{2} \backslash\left\{p_{1}, \ldots, p_{n}\right\} \rightarrow \mathbb{R}^{4}$ be the complete minimal surface $\vec{\Psi}: S^{2} \rightarrow \mathbb{R}^{4}$ is the inversion, which we assume centred at $0 \in \mathbb{R}^{4}$ up to translation. Thanks to the argument of [28], for all normal variation $\vec{v} \in$ $\mathscr{E}_{\vec{\Psi}}\left(S^{2}, T \mathbb{R}^{4}\right)$, we have

$$
\begin{aligned}
D^{2} W(\vec{\Psi})(\vec{v}, \vec{v})= & \int_{S^{2} \backslash\left\{p_{1}, \ldots, p_{n}\right\}}\left\{\frac{1}{2}\left|\Delta_{g}^{\perp} \vec{w}+\mathscr{A}(\vec{w})\right|^{2} d \operatorname{vol}_{g}\right. \\
& \left.-d \operatorname{Im}\left(2\left\langle\Delta_{g}^{\perp} \vec{w}-\mathscr{A}(\vec{w}), \partial \vec{w}\right\rangle-\partial\left(\left|\nabla^{\perp} \vec{w}\right|_{g}^{2}\right)\right)\right\},
\end{aligned}
$$

where

$$
\vec{w}=\mathscr{I}_{\vec{\Phi}}(\vec{v})=|\vec{\Phi}|^{2} \vec{v}-2\langle\vec{\Phi}, \vec{v}\rangle \vec{\Phi} .
$$

Then at every end, we have an expansion (up to translation)

$$
\vec{\Phi}(z)=\operatorname{Re}\left(\frac{\vec{A}_{0}}{z}\right)+O(|z|)
$$

for some $\vec{A}_{0} \in \mathbb{C}^{4} \backslash\{0\}$. Then $\left\langle\vec{A}_{0}, \vec{A}_{0}\right\rangle=0$ and we find that for some $\alpha_{j}>0$

$$
|\vec{\Phi}|^{2}=\frac{\alpha_{j}^{2}}{|z|^{2}}+O(|z|) .
$$

Thanks to the Sobolev embedding $W^{2,2}\left(S^{2}\right) \rightarrow C^{0}\left(S^{2}\right)$ and as $W^{2,2}\left(S^{2}\right)$ does not embed in $C^{1}\left(S^{2}\right)$ in general, we deduce that for all smooth $\vec{v} \in \mathscr{E} \Psi\left(S^{2}, T \mathbb{R}^{4}\right)$, the residue

$$
\int_{\partial B_{\varepsilon}\left(p_{j}\right)} \operatorname{Im}\left(2\left\langle\Delta_{g}^{\perp} \vec{w}-\mathscr{A}(\vec{w}), \partial \vec{w}\right\rangle-\partial\left(\left|\nabla^{\perp} \vec{w}\right|_{g}^{2}\right)\right)
$$


only depend on $\alpha_{j}, \varepsilon>0$ and $\vec{v}\left(p_{j}\right)$, up to a negligible term as $\varepsilon \rightarrow 0$ (it cannot depend on higher derivatives of $\vec{v}$ at $p_{j}$ ). Furthermore, as we can assume $\vec{v}$ to be a normal variation, we deduce that $\left\langle\vec{A}_{0}, \vec{v}\left(p_{j}\right)\right\rangle=0$. In particular, we deduce that

$$
\begin{aligned}
& \int_{\partial B_{\varepsilon}\left(p_{j}\right)} \operatorname{Im}\left(2\left\langle\Delta_{g}^{\perp} \vec{w}-\mathscr{A}(\vec{w}), \partial \vec{w}\right\rangle-\partial\left(\left|\nabla^{\perp} \vec{w}\right|_{g}^{2}\right)\right) \\
& =\left|\vec{v}\left(p_{j}\right)\right|^{2} \operatorname{Im} \int_{\partial B_{\varepsilon}\left(p_{j}\right)} 2 \Delta_{g}|\vec{\Phi}|^{2} \partial|\vec{\Phi}|^{2}-\partial\left(\left.\left.|d| \vec{\Phi}\right|^{2}\right|_{g} ^{2}\right)+o_{\varepsilon}(1) \\
& \quad=8 \pi \alpha_{j}^{2}\left|\vec{v}\left(p_{j}\right)\right|^{2}+o_{\varepsilon}(1)
\end{aligned}
$$

by the same computation as in Theorem 6.2. The rest of the proof follows [28].

Here, we show as in [28] that there is a well-defined notion of residues at ends of embedded minimal surfaces in arbitrary codimension.

Proposition 9.2 Let $\Sigma$ be a closed Riemann surface and $\vec{\Phi}: \Sigma \backslash\left\{p_{1}, \ldots, p_{n}\right\} \rightarrow \mathbb{R}^{m}$ be a complete minimal surface with embedded planar ends. Fix a covering $\left(U_{1}, \ldots, U_{n}\right)$ of $\left\{p_{1}, \ldots, p_{n}\right\} \subset \mathbb{C}$. Then the limit

$$
\lim _{\varepsilon \rightarrow 0}\left(-\frac{\varepsilon^{2}}{4 \pi} \int_{\partial B_{\varepsilon}\left(p_{i}\right)} * d\left(4|\vec{\Phi}|^{2}-\frac{1}{2}|d| \vec{\Phi}||_{g}^{2}\right)\right)
$$

is a positive real number independent depending only on $\left(U_{1}, \ldots, U_{n}\right)$ and $\vec{\Phi}$ and we denote it $\operatorname{Res}_{p_{j}}\left(\Sigma, U_{j}\right)$.

Proof As the ends are embedded and planar, there exists $\vec{A}_{0} \in \mathbb{C}^{n} \backslash\{0\}, \vec{B}_{0} \in \mathbb{C}^{n}$, and $\vec{C}_{0} \in \mathbb{R}^{n}$ we can assume that

$$
\vec{\Phi}(z)=2 \operatorname{Re}\left(\frac{\vec{A}_{0}}{z}+\vec{B}_{0} z\right)+\vec{C}_{0}+O\left(|z|^{2}\right)
$$

and we obtain

$$
\partial_{z} \vec{\Phi}=-\frac{\vec{A}_{0}}{z^{2}}+\vec{B}_{0}+O(|z|)
$$

and as $\vec{\Phi}$ is conformal, we have

$$
0=\left\langle\partial_{z} \vec{\Phi}, \partial_{z} \vec{\Phi}\right\rangle=\frac{\left\langle\vec{A}_{0}, \vec{A}_{0}\right\rangle}{z^{4}}-2 \frac{\left\langle\vec{A}_{0}, \vec{B}_{0}\right\rangle}{z^{2}}+O\left(\frac{1}{|z|}\right),
$$

which implies that

$$
\left\langle\vec{A}_{0}, \vec{A}_{0}\right\rangle=\left\langle\vec{A}_{0}, \vec{B}_{0}\right\rangle=0 .
$$

In particular, we obtain

$$
|\vec{\Phi}(z)|^{2}=\frac{2\left|\vec{A}_{0}\right|^{2}}{|z|^{2}}+4 \operatorname{Re}\left(\frac{\left\langle\vec{A}_{0}, \vec{C}_{0}\right\rangle}{z}\right)+2 \operatorname{Re}\left(\left\langle\vec{A}_{0}, \overline{\vec{B}}_{0}\right\rangle \frac{\bar{z}}{z}\right)+O(|z|) .
$$

Now, to simplify notations, write

$$
\alpha^{2}=\left|\vec{A}_{0}\right|^{2}, \quad \beta=\left\langle\vec{A}_{0}, \vec{B}_{0}\right\rangle, \quad \gamma=\left\langle\vec{A}_{0}, \vec{C}_{0}\right\rangle,
$$


which implies that

$$
|\vec{\Phi}(z)|^{2}=\frac{2 \alpha^{2}}{|z|^{2}}+4 \operatorname{Re}\left(\frac{\gamma}{z}\right)+2\left(\beta \frac{z}{\bar{z}}\right)+O(|z|)
$$

We obtain

$$
\begin{aligned}
\partial|\vec{\Phi}(z)|^{2} & =\left(-\frac{2 \alpha^{2}}{z|z|^{2}}-\frac{2 \gamma}{z^{2}}+\frac{\beta}{\bar{z}}-\bar{\beta} \frac{\bar{z}}{z^{2}}+O(1)\right) d z \\
& =-\frac{2 \alpha^{2}}{|z|^{2}}\left(\frac{1}{z}-\frac{\gamma}{\alpha^{2}} \frac{\bar{z}}{z}+\frac{\beta}{2 \alpha^{2}} z-\frac{\bar{\beta}}{2 \alpha^{2}} \frac{\bar{z}^{2}}{z}+O\left(|z|^{2}\right)\right) d z .
\end{aligned}
$$

Therefore, we have

$$
\begin{aligned}
\left.\left.|\partial| \vec{\Phi}\right|^{2}\right|^{2} & =\frac{4 \alpha^{4}}{|z|^{4}}\left(\frac{1}{|z|^{2}}-2 \operatorname{Re}\left(\frac{\gamma}{\alpha^{2}} \frac{1}{z}\right)+2 \operatorname{Re}\left(\frac{\beta}{\alpha^{2}} \frac{z}{\bar{z}}\right)-2 \operatorname{Re}\left(\frac{\bar{\beta}}{\alpha^{2}} \frac{\bar{z}}{z}\right)+O(|z|)\right) \\
& =\frac{4 \alpha^{4}}{|z|^{6}}\left(1-2 \operatorname{Re}\left(\frac{\gamma}{\alpha^{2}} \bar{z}\right)+O\left(|z|^{3}\right)\right)
\end{aligned}
$$

We also compute

$$
\begin{aligned}
e^{2 \lambda} & =2\left|\partial_{z} \vec{\Phi}\right|^{2}=\frac{2\left|\vec{A}_{0}\right|^{2}}{|z|^{4}}-4 \operatorname{Re}\left(\frac{\left\langle\vec{A}_{0}, \overrightarrow{\vec{B}_{0}}\right\rangle}{z^{2}}\right)+O\left(\frac{1}{|z|}\right) \\
& =\frac{2 \alpha^{2}}{|z|^{4}}\left(1-2 \operatorname{Re}\left(\frac{\beta}{\alpha^{2}} z^{2}\right)+O\left(|z|^{3}\right)\right)
\end{aligned}
$$

and we obtain finally by

$$
\begin{aligned}
\left.\left.|d| \vec{\Phi}\right|^{2}\right|_{g} ^{2}= & \left.\left.4 e^{-2 \lambda}|\partial| \vec{\Phi}\right|^{2}\right|^{2}=\frac{2|z|^{4}}{\alpha^{2}}\left(1+2 \operatorname{Re}\left(\frac{\beta}{\alpha^{2}} z^{2}\right)+O\left(|z|^{3}\right)\right) \\
& \times \frac{4 \alpha^{4}}{|z|^{6}}\left(1-2 \operatorname{Re}\left(\frac{\gamma}{\alpha^{2}} \bar{z}\right)+O\left(|z|^{3}\right)\right) \\
= & \frac{8 \alpha^{2}}{|z|^{2}}\left(1-2 \operatorname{Re}\left(\frac{\gamma}{\alpha^{2}} \bar{z}\right)+2 \operatorname{Re}\left(\frac{\beta}{\alpha^{2}} z^{2}\right)+O\left(|z|^{3}\right)\right) \\
= & \frac{8 \alpha^{2}}{|z|^{2}}-16 \operatorname{Re}\left(\frac{\gamma}{z}\right)+16 \operatorname{Re}\left(\beta \frac{z}{\bar{z}}\right)+O(|z|) .
\end{aligned}
$$

Therefore, we have

$$
\begin{aligned}
4|\vec{\Phi}|^{2}-\left.\left.\frac{1}{2}|d| \vec{\Phi}\right|^{2}\right|_{g} ^{2}= & 4\left(\frac{2 \alpha^{2}}{|z|^{2}}+4 \operatorname{Re}\left(\frac{\gamma}{z}\right)+2\left(\beta \frac{z}{\bar{z}}\right)+O(|z|)\right) \\
& -\frac{1}{2}\left(\frac{8 \alpha^{2}}{|z|^{2}}-16 \operatorname{Re}\left(\frac{\gamma}{z}\right)+16 \operatorname{Re}\left(\beta \frac{z}{\bar{z}}\right)+O(|z|)\right) \\
& =\frac{4 \alpha^{2}}{|z|^{2}}+24 \operatorname{Re}\left(\frac{\gamma}{z}\right)+O(|z|) .
\end{aligned}
$$

Therefore, we obtain

$$
\partial\left(4|\vec{\Phi}|^{2}-\left.\left.\frac{1}{2}|d| \vec{\Phi}\right|^{2}\right|_{g} ^{2}\right)=-\frac{4 \alpha^{2}}{|z|^{2}} \frac{d z}{z}-24 \gamma \frac{d z}{z^{2}}+O(1)
$$


which implies that

$$
\operatorname{Im} \int_{S^{1}(0, \varepsilon)} \partial\left(4|\vec{\Phi}|^{2}-\left.\left.\frac{1}{2}|d| \vec{\Phi}\right|^{2}\right|_{g} ^{2}\right)=-8 \pi \frac{\alpha^{2}}{\varepsilon^{2}}+O(\varepsilon) .
$$

Therefore, we obtain

$$
\lim _{\varepsilon \rightarrow 0}\left(-\frac{\varepsilon^{2}}{4 \pi} \int_{S^{1}(0, \varepsilon)} * d\left(4|\vec{\Phi}|^{2}-\left.\left.\frac{1}{2}|d| \vec{\Phi}\right|^{2}\right|_{g} ^{2}\right)\right)=4 \alpha^{2}>0,
$$

and concludes the proof of the Proposition.

Remark 9.3 Although this quantity is independent on the coordinate, it depends on the covering $\left\{U_{1}, \ldots, U_{n}\right\}$ of $\left\{p_{1}, \ldots, p_{n}\right\} \subset \Sigma$.

Acknowledgements I would like to thank my advisor Tristan Rivière for his constant support and for suggesting the analogy with the renormalised energy appearing in the Ginzburg-Landau model from super-conductivity (see for example $[4,32,40]$ ). I would also like thank the anonymous referee for the careful reading of the manuscript and his recommendations that permitted to increase the presentation of the article.

Open Access This article is licensed under a Creative Commons Attribution 4.0 International License, which permits use, sharing, adaptation, distribution and reproduction in any medium or format, as long as you give appropriate credit to the original author(s) and the source, provide a link to the Creative Commons licence, and indicate if changes were made. The images or other third party material in this article are included in the article's Creative Commons licence, unless indicated otherwise in a credit line to the material. If material is not included in the article's Creative Commons licence and your intended use is not permitted by statutory regulation or exceeds the permitted use, you will need to obtain permission directly from the copyright holder. To view a copy of this licence, visit http://creativecommons.org/licenses/by/4.0/.

\section{Appendix}

\subsection{Estimates for some weighted elliptic operators}

We fix an integer $m \geq 2$. Let $\omega: \mathbb{R}^{n} \rightarrow \mathbb{R}_{+}$a measurable function and for all $k \in \mathbb{N}$ and $1 \leq p<\infty$ define the weighted Sobolev space

$$
W_{\omega}^{k, p}\left(\mathbb{R}^{m}\right)=L^{p}\left(\mathbb{R}^{m}\right) \cap\left\{u:\|u\|_{W_{\omega}^{k, p}}<\infty\right\}
$$

where

$$
\|u\|_{W_{\omega}^{k, p}}=\left(\int_{\mathbb{R}^{m}}|u|^{p} d \mathscr{L}^{m}+\sum_{j=0}^{k} \int_{\mathbb{R}^{m}}\left|\nabla^{j} u\right|^{p} \omega^{p(k-j)} d \mathscr{L}^{m}\right)^{\frac{1}{p}} .
$$

By the classical Gagliardo-Nirenberg inequality, we have a continuous injection $W_{\omega}^{k, p}\left(\mathbb{R}^{m}\right) \hookrightarrow$ $W^{k, p}\left(\mathbb{R}^{m}\right)$.

Lemma 10.1 Let $\delta>0$ be a fixed real number. For all $u \in W^{2,2}\left(\mathbb{R}^{m} \backslash \bar{B}_{\delta}(0)\right)$ such that either $u=0$ or $\partial_{\nu} u=0$ on $\partial B_{\delta}(0)$, for all $1 \leq \alpha<\infty$ we have

$$
\left\|\frac{\nabla u}{|x|^{\alpha}}\right\|_{L^{2}\left(\mathbb{R}^{m} \backslash \bar{B}_{\delta}(0)\right)} \leq 2 \alpha\left\|\frac{u}{|x|^{\alpha+1}}\right\|_{L^{2}\left(\mathbb{R}^{m} \backslash \bar{B}_{\delta}(0)\right)}+\left\|\frac{u}{|x|^{\alpha+1}}\right\|_{L^{2}\left(\mathbb{R}^{m} \backslash \bar{B}_{\delta}(0)\right)}^{\frac{1}{2}}\left\|\frac{\Delta u}{|x|^{\alpha-1}}\right\|_{L^{2}\left(\mathbb{R}^{m} \backslash \bar{B}_{\delta}(0)\right)}^{\frac{1}{2}}
$$


provided that the integrals on the right-hand side of (10.1) are finite. In particular, if $\omega$ : $\mathbb{R}^{n} \rightarrow \mathbb{R}$ is such that $\omega(x)=|x|^{-1}$, we have a continuous injection

$$
W_{0,0}^{2,2}\left(\mathbb{R}^{m} \backslash \bar{B}_{\delta}(0)\right) \cap L_{\omega}^{2}\left(\mathbb{R}^{m} \backslash \bar{B}_{\delta}(0)\right) \hookrightarrow W_{\omega}^{2,2}\left(\mathbb{R}^{m} \backslash \bar{B}_{\delta}(0)\right),
$$

where $W_{0,0}^{2,2}\left(\mathbb{R}^{m} \backslash \bar{B}(0, \delta)\right)=W^{2,2}\left(\mathbb{R}^{m} \backslash \bar{B}_{\delta}(0)\right) \cap\left\{u: u \partial_{\nu} u=0\right.$ on $\left.\partial B(0, \delta)\right\}$.

Proof We first assume that $u \in W^{2,2} \cap C^{\infty}\left(\mathbb{R}^{m} \backslash \bar{B}_{\delta}(0)\right)$ is such that either $u=0$ or $\partial_{\nu} u=0$ on $\partial_{\nu} u=0$ on $\partial B_{\delta}(0)$ (so that $u \partial_{\nu} u=0$ on $\partial B_{\delta}(0)$ ). Then we have

$$
\operatorname{div}\left(u \nabla u|x|^{-2 \alpha}\right)=|\nabla u|^{2}|x|^{-2 \alpha}+u \Delta u|x|^{-2 \alpha}-2 \alpha u(\nabla u \cdot x)|x|^{-2(\alpha+1)}
$$

Therefore, fixing $0 \leq \theta_{1}, \theta_{2} \leq 1,1<p<\infty$, we have by the Cauchy-Schwarz inequality and since $u \partial_{v} u=0$ on $\partial B_{\delta}(0)$ the identity

$$
\begin{aligned}
\int_{\mathbb{R}^{m} \backslash \bar{B}_{\delta}(0)} \frac{|\nabla u|^{2}}{|x|^{2 \alpha}} d x= & 2 \alpha \int_{\mathbb{R}^{m} \backslash \bar{B}_{\delta}(0)} u \frac{\nabla u \cdot x}{|x|^{2(\alpha+1)}} d x-\int_{\mathbb{R}^{m} \backslash \bar{B}_{\delta}(0)} \frac{u \Delta u}{|x|^{2 \alpha}} d x \\
& \leq 2 \alpha\left(\int_{\mathbb{R}^{m} \backslash \bar{B}_{\delta}(0)} \frac{u^{2}}{|x|^{2(2 \alpha+1) \theta_{1}}} d x\right)^{\frac{1}{2}}\left(\int_{\mathbb{R}^{m} \backslash \bar{B}_{\delta}(0)} \frac{|\nabla u|^{2}}{|x|^{2(2 \alpha+1)\left(1-\theta_{1}\right)}} d x\right)^{\frac{1}{2}} \\
& +\left(\int_{\mathbb{R}^{m} \backslash \bar{B}_{\delta}(0)} \frac{u^{2}}{|x|^{4 \alpha \theta_{2}}} d x\right)^{\frac{1}{2}}\left(\int_{\mathbb{R}^{m} \backslash \bar{B}_{\delta}(0)} \frac{(\Delta u)^{2}}{|x|^{4 \alpha\left(1-\theta_{2}\right)}} d x\right)^{\frac{1}{2}}
\end{aligned}
$$

As we want to recover the same exponent for $|x|$ in the denominator of $u^{2}$ (and $|\nabla u|^{2}$ ) on both sides, we choose $\theta_{1}$ such that

$$
(2 \alpha+1)\left(1-\theta_{1}\right)=\alpha
$$

i.e.

$$
\theta_{1}=\frac{\alpha+1}{2 \alpha+1}
$$

and $\theta_{2}$ such that

$$
2 \alpha \theta_{2}=(2 \alpha+1) \theta_{1}
$$

so

$$
\theta_{2}=\frac{\alpha+1}{2 \alpha} \in[0,1]
$$

for all $\alpha \geq 1$. Finally, we get if

$$
\begin{gathered}
X=\left(\int_{\mathbb{R}^{m} \backslash \bar{B}_{\delta}(0)} \frac{|\nabla u|^{2}}{|x|^{2 \alpha}} d x\right)^{\frac{1}{2}}, \quad a=2 \alpha\left(\int_{\mathbb{R}^{m} \backslash \bar{B}_{\delta}(0)} \frac{u^{2}}{|x|^{2(\alpha+1)}} d x\right)^{\frac{1}{2}}, \\
b=\left(\int_{\mathbb{R}^{m} \backslash \bar{B}_{\delta}(0)} \frac{u^{2}}{|x|^{2(\alpha+1)}} d x\right)^{\frac{1}{2}}\left(\int_{\mathbb{R}^{m} \backslash \bar{B}_{\delta}(0)} \frac{(\Delta u)^{2}}{|x|^{2(\alpha-1)}} d x\right)^{\frac{1}{2}} \\
X^{2} \leq a X+b
\end{gathered}
$$

Therefore,

$$
X \leq \frac{1}{2}\left(a+\sqrt{a^{2}+4 b}\right) \leq a+\sqrt{b},
$$


or

$$
\left\|\frac{\nabla u}{|x|^{\alpha}}\right\|_{\mathrm{L}^{2}\left(\mathbb{R}^{m} \backslash \bar{B}_{\delta}(0)\right)} \leq 2 \alpha\left\|\frac{u}{|x|^{\alpha+1}}\right\|_{\mathrm{L}^{2}\left(\mathbb{R}^{m} \backslash \bar{B}_{\delta}(0)\right)}+\left\|\frac{u}{|x|^{\alpha+1}}\right\|_{\mathrm{L}^{2}\left(\mathbb{R}^{m} \backslash \bar{B}_{\delta}(0)\right)}^{\frac{1}{2}}\left\|\frac{\Delta u}{|x|^{\alpha-1}}\right\|_{\mathrm{L}^{2}\left(\mathbb{R}^{m} \backslash \bar{B}_{\delta}(0)\right)}^{\frac{1}{2}} .
$$

Notice that this inequality cannot be improved by a scaling argument because of the singular weights. The general inequality for $u \in W^{2,2}\left(\mathbb{R}^{2} \backslash \bar{B}_{\delta}(0)\right)$ such that either $u=0$ or $\partial_{\nu} u=0$ on $\partial B_{\delta}(0)$ follows by standard regularisation. This concludes the proof of the lemma.

Lemma 10.2 Let $\delta>0$ be a fixed real number, and define for all for all $m \geq 1$ the second order elliptic differential operator

$$
\mathscr{L}_{m}=\Delta-2(m+1) \frac{x}{|x|^{2}} \cdot \nabla+\frac{(m+1)^{2}}{|x|^{2}} .
$$

Let $u \in W^{2,2}\left(\mathbb{R}^{2} \backslash \bar{B}_{\delta}(0)\right)$ be such that $u=\partial_{\nu} u=0$ on $\partial B_{\delta}(0)$ and assume that $\mathscr{L}_{m} u \in$ $L^{2}\left(\mathbb{R}^{2}\right)$. Then we have the identities

$$
\begin{aligned}
\int_{\mathbb{R}^{2} \backslash \bar{B}_{\delta}(0)}\left(\mathscr{L}_{m} u\right)^{2} d x= & \int_{\mathbb{R}^{2} \backslash \bar{B}_{\delta}(0)}\left(\Delta u-2(m+1) \frac{x}{|x|^{2}} \cdot \nabla u+(m+1)^{2} \frac{u}{|x|^{2}}\right)^{2} d x \\
= & \int_{\mathbb{R}^{2} \backslash \bar{B}_{\delta}(0)}\left(\Delta u+(m+1)(m-1) \frac{u}{|x|^{2}}\right)^{2} d x \\
& +4(m+1)(m-1) \int_{\mathbb{R}^{2} \backslash \bar{B}_{\delta}(0)}\left(\frac{x}{|x|^{2}} \cdot \nabla u-\frac{u}{|x|^{2}}\right)^{2} d x
\end{aligned}
$$

In particular, we have for $m=1$

$$
\begin{aligned}
\int_{\mathbb{R}^{2} \backslash \bar{B}_{\delta}(0)}\left(\mathscr{L}_{1} u\right)^{2} d x & =\int_{\mathbb{R}^{2} \backslash \bar{B}_{\delta}(0)}\left(\Delta u-4 \frac{x}{|x|^{2}} \cdot \nabla u+\frac{4}{|x|^{2}} u\right)^{2} d x \\
& =\int_{\mathbb{R}^{2} \backslash \bar{B}_{\delta}(0)}(\Delta u)^{2} d x .
\end{aligned}
$$

Furthermore, if $m>1$, then

$$
\begin{aligned}
& \left\|\frac{u}{|x|^{2}}\right\|_{\mathrm{L}^{2}\left(\mathbb{R}^{2} \backslash \bar{B}_{\delta}(0)\right)} \leq \frac{1}{(m+1)(m-1)}\|\Delta u\|_{\mathrm{L}^{2}\left(\mathbb{R}^{2} \backslash \bar{B}_{\delta}(0)\right)} \\
& +\frac{1}{(m+1)(m-1)}\left\|\mathscr{L}_{m} u\right\|_{\mathrm{L}^{2}\left(\mathbb{R}^{2} \backslash \bar{B}_{\delta}(0)\right)} .
\end{aligned}
$$

If $m \geq 3$,

$$
\left\|\frac{\nabla u \cdot x}{|x|^{2}}\right\|_{\mathrm{L}^{2}\left(\mathbb{R}^{2} \backslash \bar{B}_{\delta}(0)\right)} \leq \frac{1}{2(m+1)}\|\Delta u\|_{\mathrm{L}^{2}\left(\mathbb{R}^{2} \backslash \bar{B}_{\delta}(0)\right)}+\frac{1}{2(m+1)}\left\|\mathscr{L}_{m} u\right\|_{\mathrm{L}^{2}\left(\mathbb{R}^{2} \backslash \bar{B}_{\delta}(0)\right)}
$$

while for $1<m \leq 3$

$$
\begin{aligned}
\left\|\frac{\nabla u \cdot x}{|x|^{2}}\right\|_{\mathrm{L}^{2}\left(\mathbb{R}^{2} \backslash \bar{B}_{\delta}(0)\right)} \leq & \frac{1}{(m+1)(m-1)}\|\Delta u\|_{\mathrm{L}^{2}\left(\mathbb{R}^{2} \backslash \bar{B}_{\delta}(0)\right)} \\
& +\frac{1}{(m+1)(m-1)}\left\|\mathscr{L}_{m}\right\|_{\mathrm{L}^{2}\left(\mathbb{R}^{2} \backslash \bar{B}_{\delta}(0)\right)} .
\end{aligned}
$$


Proof Step 1: Equalities. Observe that for all $x \in \mathbb{R}^{2} \backslash\{0\}$, we have

$$
\Delta \frac{1}{|x|^{2 \alpha}}=\frac{4 \alpha^{2}}{|x|^{2 \alpha+2}}
$$

and assuming that $u \in W^{2,2} \cap C^{\infty}\left(\mathbb{R}^{2} \backslash \bar{B}_{\delta}(0)\right)$ without loss of generality, we have since $\Delta u^{2}=2 u \Delta u+2|\nabla u|^{2}$

$$
\begin{aligned}
\int_{\mathbb{R}^{2} \backslash \bar{B}_{\delta}(0)} \frac{u^{2}}{|x|^{2 \alpha+2}} d x & =\int_{\mathbb{R}^{2} \backslash \bar{B}_{\delta}(0)} u^{2} \frac{1}{4 \alpha^{2}} \Delta \frac{1}{|x|^{2 \alpha}} d x=\int_{\mathbb{R}^{2} \backslash \bar{B}_{\delta}(0)} \frac{\Delta u^{2}}{4 \alpha^{2}|x|^{2 \alpha}} d x \\
& =\int_{\mathbb{R}^{2} \backslash \bar{B}_{\delta}(0)} \frac{u \Delta u+|\nabla u|^{2}}{2 \alpha^{2}|x|^{2 \alpha}} d x
\end{aligned}
$$

Furthermore, recall that by (10.3)

$$
\int_{\mathbb{R}^{2} \backslash \bar{B}_{\delta}(0)} \frac{u \Delta u+|\nabla u|^{2}}{|x|^{2 \alpha}} d x=\int_{\mathbb{R}^{2} \backslash \bar{B}_{\delta}(0)} 2 \alpha \frac{u(\nabla u \cdot x)}{|x|^{2 \alpha+2}} d x .
$$

Therefore, we find

$$
\begin{aligned}
\int_{\mathbb{R}^{2} \backslash \bar{B}_{\delta}(0)} \frac{u^{2}}{|x|^{2 \alpha+2}} d x & =\frac{1}{\alpha} \int_{\mathbb{R}^{2} \backslash \bar{B}_{\delta}(0)} \frac{u(\nabla u \cdot x))}{|x|^{2 \alpha+2}} d x \\
& \leq \frac{1}{\alpha}\left(\int_{\mathbb{R}^{2} \backslash \bar{B}_{\delta}(0)} \frac{u^{2}}{|x|^{2 \alpha+2}} d x\right)^{\frac{1}{2}}\left(\int_{\mathbb{R}^{2} \backslash \bar{B}_{\delta}(0)} \frac{(\nabla u \cdot x)^{2}}{|x|^{2 \alpha+2}} d x\right)^{\frac{1}{2}}
\end{aligned}
$$

which implies that

$$
\left\|\frac{u}{|x|^{\alpha+1}}\right\|_{\mathrm{L}^{2}\left(\mathbb{R}^{2} \backslash \bar{B}_{\delta}(0)\right)} \leq \frac{1}{\alpha}\left\|\frac{\nabla u \cdot x}{|x|^{\alpha+1}}\right\|_{\mathrm{L}^{2}\left(\mathbb{R}^{2} \backslash \bar{B}_{\delta}(0)\right)} .
$$

Now, if $\alpha=1$, we find equivalently

$$
\begin{aligned}
& \int_{\mathbb{R}^{2} \backslash \bar{B}_{\delta}(0)} \frac{u^{2}}{|x|^{4}} d x=\int_{\mathbb{R}^{2} \backslash \bar{B}_{\delta}(0)} \frac{u(\nabla u \cdot x)}{|x|^{4}} d x \\
& \int_{\mathbb{R}^{2} \backslash \bar{B}_{\delta}(0)} \frac{u}{|x|^{2}}\left(\frac{u}{|x|^{2}}-\frac{\nabla u \cdot x}{|x|^{2}}\right) d x=0 .
\end{aligned}
$$

Now, compute for all $u \in C_{c}^{\infty}\left(\mathbb{R}^{2}\right)$

$$
\begin{aligned}
\Delta\left(\frac{u}{|x|^{2}}\right) & =\frac{1}{|x|^{2}} \Delta u+2 \nabla\left(\frac{1}{|x|^{2}}\right) \cdot \nabla u+u \Delta\left(\frac{1}{|x|^{2}}\right) \\
& =\frac{1}{|x|^{2}}\left(\Delta-4 \frac{x}{|x|^{2}} \cdot \nabla+\frac{4}{|x|^{2}}\right) u .
\end{aligned}
$$

We also have

$$
\begin{aligned}
& \partial_{x_{1}}\left(\frac{x_{1}}{|x|^{2}} \partial_{x_{1}} u\right)=\left(\frac{1}{|x|^{2}}-\frac{2 x_{1}^{2}}{|x|^{4}}\right) \partial_{x_{1}} u+\frac{1}{|x|^{2}} \partial_{x_{1}}^{2} u \\
& \partial_{x_{1}}^{2}\left(\frac{x_{1}}{|x|^{2}} \partial_{x_{1}} u\right)=\left(-\frac{6 x_{1}}{|x|^{4}}+\frac{8 x_{1}^{3}}{|x|^{6}}\right) \partial_{x_{1}} u+2\left(\frac{1}{|x|^{2}}-\frac{2 x_{1}^{2}}{|x|^{4}}\right) \partial_{x_{1}}^{2} u+\frac{x_{1}}{|x|^{2}} \partial_{x_{1}}^{3} u \\
& \partial_{x_{2}}\left(\frac{x_{1}}{|x|^{2}} \partial_{x_{1}} u\right)=-\frac{2 x_{1} x_{2}}{|x|^{4}} \partial_{x_{1}} u+\frac{x_{1}}{|x|^{2}} \partial_{x_{1}, x_{2}}^{2} u
\end{aligned}
$$




$$
\partial_{x_{2}}^{2}\left(\frac{x_{1}}{|x|^{2}} \partial_{x_{1}} u\right)=\left(-\frac{2 x_{1}}{|x|^{4}}+\frac{8 x_{1} x_{2}^{2}}{|x|^{6}}\right) \partial_{x_{1}} u-\frac{4 x_{1} x_{2}}{|x|^{4}} \partial_{x_{1}, x_{2}}^{2} u+\frac{x_{1}}{|x|^{2}} \partial_{x_{1}} \partial_{x_{2}}^{2} u
$$

Therefore, we find

$$
\begin{aligned}
\Delta\left(\frac{x_{1}}{|x|^{2}} \partial_{x_{1}} u\right)= & \left(-\frac{8 x_{1}}{|x|^{4}}+\frac{8 x_{1}\left(x_{1}^{2}+x_{2}^{2}\right)}{|x|^{6}}\right) \partial_{x_{1}} u+\frac{x_{1}}{|x|^{2}} \partial_{x_{1}}\left(\partial_{x_{1}}^{2} u+\partial_{x_{2}}^{2} u\right)+\frac{2}{|x|^{2}} \partial_{x_{1}}^{2} u \\
& -4\left(\frac{x_{1}^{2}}{|x|^{4}} \partial_{x_{1}}^{2} u+\frac{x_{1} x_{2}}{|x|^{4}} \partial_{x_{1}, x_{2}}^{2} u\right) \\
= & \frac{x_{1}}{|x|^{2}} \partial_{x_{1}} \Delta u+\frac{2}{|x|^{2}} \partial_{x_{1}}^{2} u-4\left(\frac{x_{1}^{2}}{|x|^{4}} \partial_{x_{1}}^{2} u+\frac{x_{1} x_{2}}{|x|^{4}} \partial_{x_{1}, x_{2}}^{2} u\right),
\end{aligned}
$$

and by symmetry this implies that

$$
\begin{aligned}
\Delta\left(\frac{x}{|x|^{2}} \cdot \nabla u\right) & =\frac{x}{|x|^{2}} \cdot \nabla \Delta u+\frac{2}{|x|^{2}} \Delta u-4\left(\frac{x_{1}^{2}}{|x|^{4}} \partial_{x_{1}}^{2} u+\frac{2 x_{1} x_{2}}{|x|^{4}} \partial_{x_{1}, x_{2}}^{2} u+\frac{x_{2}^{2}}{|x|^{4}} \partial_{x_{2}}^{2} u\right) \\
& =\frac{x}{|x|^{2}} \cdot \nabla \Delta u+\frac{2}{|x|^{2}} \Delta u-4\left(\frac{x}{|x|^{2}}\right)^{t} \cdot \nabla^{2} u \cdot\left(\frac{x}{|x|^{2}}\right) .
\end{aligned}
$$

Therefore, we deduce that

$$
\begin{aligned}
\Delta\left(\mathscr{L}_{m}\right)= & \Delta^{2}-2(m+1) \frac{x}{|x|^{2}} \cdot \nabla \Delta u-\frac{4(m+1)}{|x|^{2}} \Delta u+8(m+1)\left(\frac{x}{|x|^{2}}\right)^{t} \cdot \nabla^{2} u \cdot\left(\frac{x}{|x|^{2}}\right) \\
& +\frac{(m+1)^{2}}{|x|^{2}}\left(\Delta u-4 \frac{x}{|x|^{2}} \cdot \nabla u+\frac{4}{|x|^{2}} u\right) \\
= & \Delta^{2}-2(m+1) \frac{x}{|x|^{2}} \cdot \nabla \Delta u+\frac{(m+1)^{2}-4(m+1)}{|x|^{2}} \Delta u \\
& +8(m+1)\left(\frac{x}{|x|^{2}}\right)^{t} \cdot \nabla^{2} u \cdot\left(\frac{x}{|x|^{2}}\right) \\
& -4(m+1)^{2} \frac{x}{|x|^{4}} \cdot \nabla u+\frac{4(m+1)^{2}}{|x|^{4}} u .
\end{aligned}
$$

Now, we have

$$
\frac{x}{|x|^{2}} \cdot \nabla\left(\frac{1}{|x|^{2}} u\right)=-\frac{2}{|x|^{4}} u+\frac{x}{|x|^{4}} \cdot \nabla u,
$$

and

$$
\begin{aligned}
x_{1} \partial_{x_{1}}\left(\frac{x}{|x|^{2}} \cdot \nabla u\right) & =x_{1}\left\{\left(\frac{1}{|x|^{2}}-\frac{2 x_{1}^{2}}{|x|^{4}}\right) \partial_{x_{1}} u-\frac{2 x_{1} x_{2}}{|x|^{4}} \partial_{x_{2}} u+\frac{x}{|x|^{4}} \cdot \nabla \partial_{x_{1}} u\right\} \\
& =\frac{x_{1}\left(-x_{1}^{2}+x_{2}^{2}\right)}{|x|^{4}} \partial_{x_{1}} u-\frac{2 x_{1}^{2} x_{2}}{|x|^{4}} \partial_{x_{2}} u+\frac{x_{1}^{2}}{|x|^{2}} \partial_{x_{1}}^{2} u+\frac{x_{1} x_{2}}{|x|^{2}} \partial_{x_{1}, x_{2}}^{2} u \\
x_{2} \partial_{x_{2}}\left(\frac{x}{|x|^{2}} \cdot \nabla u\right) & =\frac{x_{2}\left(x_{1}^{2}-x_{2}^{2}\right)}{|x|^{4}} \partial_{x_{2}} u-\frac{2 x_{1} x_{2}^{2}}{|x|^{4}} \partial_{x_{1}} u+\frac{x_{1}^{2}}{|x|^{2}} \partial_{x_{1}}^{2} u+\frac{x_{1} x_{2}}{|x|^{2}} \partial_{x_{1}, x_{2}}^{2} u \\
\frac{x}{|x|^{2}} \cdot \nabla\left(\frac{x}{|x|^{2}} \cdot \nabla u\right) & =-\frac{x}{|x|^{4}} \cdot \nabla u+\left(\frac{x}{|x|^{2}}\right)^{t} \cdot \nabla^{2} u \cdot\left(\frac{x}{|x|^{2}}\right),
\end{aligned}
$$


which implies that

$$
\begin{aligned}
\frac{x}{|x|^{2}} \cdot \nabla\left(\mathscr{L}_{m} u\right)= & \frac{x}{|x|^{2}} \cdot \nabla \Delta u-2(m+1)\left(\frac{x}{|x|^{2}}\right)^{t} \cdot \nabla^{2} u \cdot\left(\frac{x}{|x|^{2}}\right) \\
& +\left\{(m+1)^{2}+2(m+1)\right\} \frac{x}{|x|^{4}} \cdot \nabla u \\
& -\frac{2(m+1)^{2}}{|x|^{4}} u .
\end{aligned}
$$

Therefore,

$$
\begin{aligned}
\mathscr{L}_{m}^{*} \mathscr{L}_{m} u= & \left(\Delta+2(m+1) \frac{x}{|x|^{2}} \cdot \nabla+\frac{(m+1)^{2}}{|x|^{2}}\right)\left(\mathscr{L}_{m} u\right) \\
= & \Delta^{2} u-\frac{2(m+1) \frac{x}{|x|^{2}} \cdot \nabla \Delta u+\frac{(m+1)^{2}-4(m+1)}{|x|^{2}} \Delta u}{} \\
& +8(m+1)\left(\frac{x}{|x|^{2}}\right)^{t} \cdot \nabla^{2} u \cdot\left(\frac{x}{|x|^{2}}\right) \\
& -\frac{4(m+1)^{2} \frac{x}{|x|^{2}} \cdot \nabla u+\frac{4(m+1)^{2}}{|x|^{4}} u}{}+\frac{2(m+1) \frac{x}{|x|^{2}} \cdot \nabla \Delta u-4(m+1)^{2}\left(\frac{x}{|x|^{2}}\right)^{t} \cdot \nabla^{2} u \cdot\left(\frac{x}{|x|^{2}}\right)}{} \\
& +\left\{\frac{\left.2(m+1)^{3}+4(m+1)^{2}\right\} \frac{x}{|x|^{2}} \cdot \nabla u}{|x u|^{2}} \Delta u-2(m+1)^{3} \frac{x}{|x|^{2}} \cdot \nabla u+\frac{(m+1)^{4}}{|x|^{4}} u\right. \\
& -\frac{4(m+1)^{3}}{|x|^{4}} u+\frac{(m+1)^{2}}{|x|^{2}} \\
= & \Delta^{2} u+\frac{2(m+1)(m-1)}{|x|^{2}} \Delta u-4(m+1)(m-1)\left(\frac{x}{|x|^{2}}\right)^{t} \cdot \nabla^{2} u \cdot\left(\frac{x}{|x|^{2}}\right) \\
& +\frac{(m+1)^{2}(m-1)^{2}}{|x|^{4}} u,
\end{aligned}
$$

and we indeed recover $\mathscr{L}^{*} \mathscr{L}_{1}=\Delta^{2}$. We deduce that for all $u \in W^{2,2} \cap C^{\infty}\left(\mathbb{R}^{2} \backslash \bar{B}_{\delta}(0)\right)$ such that $u=\partial_{\nu} u=0$ on $\partial B_{\delta}(0)$,

$$
\begin{aligned}
& \int_{\mathbb{R}^{2} \backslash \bar{B}_{\delta}(0)}\left(\Delta u-2(m+1) \frac{x}{|x|^{2}} \cdot \nabla u+\frac{(m+1)^{2}}{|x|^{2}} u\right)^{2} d x=\int_{\mathbb{R}^{2} \backslash \bar{B}_{\delta}(0)}\left(\mathscr{L}_{m} u\right)^{2} d x \\
& =\int_{\mathbb{R}^{2} \backslash \bar{B}_{\delta}(0)}\left(u \Delta^{2} u+2(m+1)(m-1) \frac{u}{|x|^{2}} \Delta u\right. \\
& \quad-4(m+1)(m-1) u\left(\frac{x}{|x|^{2}}\right)^{t} \cdot \nabla^{2} u \cdot\left(\frac{x}{|x|^{2}}\right) \\
& \left.+\frac{(m+1)^{2}(m-1)^{2}}{|x|^{4}} u^{2}\right) d x+\int_{\partial B_{\delta}(0)}\left(u \partial_{v}\left(\mathscr{L}_{m} u\right)-\partial_{v} u\left(\mathscr{L}_{m} u\right)\right) d \mathscr{H}^{1} \\
& =\int_{\mathbb{R}^{2} \backslash \bar{B}_{\delta}(0)}\left((\Delta u)^{2}+2(m+1)(m-1) \Delta u \frac{u}{|x|^{2}}+(m+1)^{2}(m-1)^{2} \frac{u^{2}}{|x|^{4}}\right) d x \\
& \quad-4(m+1)(m-1) \int_{\mathbb{R}^{2} \backslash \bar{B}_{\delta}(0)} u\left(\frac{x}{|x|^{2}}\right)^{t} \cdot \nabla^{2} u \cdot\left(\frac{x}{|x|^{2}}\right) d x
\end{aligned}
$$




$$
\begin{aligned}
= & \int_{\mathbb{R}^{2} \backslash \bar{B}_{\delta}(0)}\left(\Delta u+(m+1)(m-1)^{2} \frac{u}{|x|^{2}}\right)^{2} d x \\
& -4(m+1)(m-1) \int_{\mathbb{R}^{2} \backslash \bar{B}_{\delta}(0)} u\left(\frac{x}{|x|^{2}}\right)^{t} \cdot \nabla^{2} u \cdot\left(\frac{x}{|x|^{2}}\right) d x .
\end{aligned}
$$

Now, observe that by (5.42)

$$
\begin{aligned}
\int_{\mathbb{R}^{2} \backslash \bar{B}_{\delta}(0)} u\left(\frac{x}{|x|^{2}}\right)^{t} \cdot \nabla^{2} u \cdot\left(\frac{x}{|x|^{2}}\right) d x \\
=\int_{\mathbb{R}^{2} \backslash \bar{B}_{\delta}(0)} \frac{x_{1}}{|x|^{2}} u\left(\frac{x_{1}}{|x|^{2}} \partial_{x_{1}}\left(\partial_{x_{1}} u\right)+\frac{x}{|x|^{2}} \partial_{x_{2}}\left(\partial_{x_{1}} u\right)\right) \\
\quad+\frac{x_{2}}{|x|^{2}} u\left(\frac{x_{1}}{|x|^{2}} \partial_{x_{1}}\left(\partial_{x_{2}} u\right)+\frac{x_{2}}{|x|^{2}} \partial_{x_{2}}\left(\partial_{x_{1}} u\right)\right) d x \\
=\int_{\mathbb{R}^{2} \backslash \bar{B}_{\delta}(0)}\left(\frac{x_{1}}{|x|^{2}} u \frac{x}{|x|^{2}} \cdot \nabla\left(\partial_{x_{1}} u\right)+\frac{x_{2}}{|x|^{2}} u \frac{x}{|x|^{2}} \cdot \nabla\left(\partial_{x_{2}} u\right)\right) d x \\
=-\int_{\mathbb{R}^{2} \backslash \bar{B}_{\delta}(0)}\left(\frac{x_{1}}{|x|^{2}}\left(\frac{x}{|x|^{2}} \cdot \nabla u\right) \partial_{x_{1}} u+\frac{x_{2}}{|x|^{2}}\left(\frac{x}{|x|^{2}} \cdot \nabla u\right) \partial_{x_{2}} u\right) d x \\
\quad+\int_{\mathbb{R}^{2} \backslash \bar{B}_{\delta}(0)} u \frac{x}{|x|^{4}} \cdot \nabla u d x \\
\quad-\int_{\partial_{B_{\delta}(0)}}\left(\frac{x_{1}}{|x|^{3}} u \partial_{\nu}\left(\partial_{x_{1}} u\right)+\frac{x_{2}}{|x|^{3}} u \partial_{\nu}\left(\partial_{x_{2}} u\right)\right) d \mathscr{H}^{1} \\
=-\int_{\mathbb{R}^{2} \backslash \bar{B}_{\delta}(0)}\left(\frac{\nabla u \cdot x}{|x|^{2}}\right)^{2} d x+\int_{\mathbb{R}^{2} \backslash \bar{B}_{\delta}(0)} u \frac{x}{|x|^{4}} \cdot \nabla u d x \leq 0
\end{aligned}
$$

where we used

$$
\nabla\left(\frac{x_{1}}{|x|^{2}}\right) \cdot \frac{x}{|x|^{2}}=\frac{1}{|x|^{2}}\left(\frac{x_{1}}{|x|^{2}}-\frac{2 x_{1}^{3}}{|x|^{4}}-\frac{2 x_{1} x_{2}^{2}}{|x|^{4}}\right)=-\frac{x_{1}}{|x|^{4}},
$$

The last inequality come from the following observations (see the computations before (10.5) for an alternative derivation)

$$
\begin{aligned}
\int_{\mathbb{R}^{2} \backslash \bar{B}_{\delta}(0)} u \frac{x}{|x|^{4}} \cdot \nabla u d x & =\int_{\mathbb{R}^{2} \backslash \bar{B}_{\delta}(0)} \frac{u}{|x|^{2}} \operatorname{div}\left(\frac{x}{|x|^{2}} u\right) d x \\
& =-\int_{\mathbb{R}^{2} \backslash \bar{B}_{\delta}(0)}\left(\left(\frac{x}{|x|^{4}} \cdot \nabla u\right) u+\nabla\left(\frac{1}{|x|^{2}}\right) \cdot \frac{x}{|x|^{2}} u^{2}\right) d x \\
& =-\int_{\mathbb{R}^{2} \backslash \bar{B}_{\delta}(0)} u \frac{x}{|x|^{4}} \cdot \nabla u d x+2 \int_{\mathbb{R}^{2} \backslash \bar{B}_{\delta}(0)} \frac{u^{2}}{|x|^{4}} d x
\end{aligned}
$$

so that

$$
\int_{\mathbb{R}^{2} \backslash \bar{B}_{\delta}(0)} \frac{u^{2}}{|x|^{4}} d x=\int_{\mathbb{R}^{2} \backslash \bar{B}_{\delta}(0)} u \frac{x}{|x|^{4}} \cdot \nabla u d x,
$$

Therefore, thanks to (10.8), we rewrite (10.7) as

$$
\int_{\mathbb{R}^{2} \backslash \bar{B}_{\delta}(0)}\left(\frac{\nabla u \cdot x}{|x|^{2}}\right)^{2} d x-\int_{\mathbb{R}^{2} \backslash \bar{B}_{\delta}(0)} u \frac{x}{|x|^{4}} \cdot \nabla u d x
$$




$$
\begin{aligned}
& =\int_{\mathbb{R}^{2} \backslash \bar{B}_{\delta}(0)}\left(\left(\frac{\nabla u \cdot x}{|x|^{2}}\right)^{2}-2\left(\frac{\nabla u \cdot x}{|x|^{2}}\right) \frac{u}{|x|^{2}}+\frac{u^{2}}{|x|^{4}}\right) d x \\
& =\int_{\mathbb{R}^{2} \backslash \bar{B}_{\delta}(0)}\left(\frac{x}{|x|^{2}} \cdot \nabla u-\frac{u}{|x|^{2}}\right)^{2} d x .
\end{aligned}
$$

Finally, we deduce by (10.6), (10.7) and (10.9) that

$$
\begin{aligned}
\int_{\mathbb{R}^{2} \backslash \bar{B}_{\delta}(0)}\left(\mathscr{L}_{m} u\right)^{2} d x & =\int_{\mathbb{R}^{2} \backslash \bar{B}_{\delta}(0)}\left(\Delta u-2(m+1) \frac{x}{|x|^{2}} \cdot \nabla u+\frac{(m+1)^{2}}{|x|^{2}} u\right)^{2} d x \\
= & \int_{\mathbb{R}^{2} \backslash \bar{B}_{\delta}(0)}\left(\Delta u+(m+1)(m-1) \frac{u}{|x|^{2}}\right)^{2} d x \\
& +4(m+1)(m-1) \int_{\mathbb{R}^{2} \backslash \bar{B}_{\delta}(0)}\left(\frac{x}{|x|^{2}} \cdot \nabla u-\frac{u}{|x|^{2}}\right)^{2} d x .
\end{aligned}
$$

Step 2: Inequalities. Now we have thanks to (10.8)

$$
\begin{aligned}
& \int_{\mathbb{R}^{2} \backslash \bar{B}_{\delta}(0)}\left(-2(m+1) \frac{x}{|x|^{2}} \cdot \nabla u+\frac{(m+1)^{2}}{|x|^{2}} u\right)^{2} d x \\
& =\int_{\mathbb{R}^{2} \backslash \bar{B}_{\delta}(0)} 4(m+1)^{2}\left(\frac{\nabla u \cdot x}{|x|^{2}}\right)^{2} d x \\
& \quad+\int_{\mathbb{R}^{2} \backslash \bar{B}_{\delta}(0)}(m+1)^{4} \frac{u^{2}}{|x|^{4}} d x-4(m+1)^{3} \int_{\mathbb{R}^{2} \backslash \bar{B}_{\delta}(0)} \frac{u(\nabla u \cdot x)}{|x|^{4}} d x \\
& =4(m+1)^{2} \int_{\mathbb{R}^{2} \backslash \bar{B}_{\delta}(0)} \frac{(\nabla u \cdot x)^{2}}{|x|^{4}} d x+\left((m+1)^{4}-4(m+1)^{3}\right) \int_{\mathbb{R}^{2} \backslash \bar{B}_{\delta}(0)} \frac{u^{2}}{|x|^{4}} d x \\
& \geq\left((m+1)^{4}+4(m+1)^{2}-4(m+1)^{3}\right) \int_{\mathbb{R}^{2} \backslash \bar{B}_{\delta}(0)} \frac{u^{2}}{|x|^{4}} d x \\
& =(m+1)^{2}(m-1)^{2} \int_{\mathbb{R}^{2} \backslash \bar{B}_{\delta}(0)} \frac{u^{2}}{|x|^{4}} d x
\end{aligned}
$$

so for $m>1$, we find

$$
\begin{aligned}
& \left\|\frac{u}{|x|^{2}}\right\|_{\mathrm{L}^{2}\left(\mathbb{R}^{2} \backslash \bar{B}_{\delta}(0)\right)} \leq \frac{1}{(m+1)(m-1)}\|\Delta u\|_{\mathrm{L}^{2}\left(\mathbb{R}^{2} \backslash \bar{B}_{\delta}(0)\right)} \\
& +\frac{1}{(m+1)(m-1)}\left\|\mathscr{L}_{m} u\right\|_{\mathrm{L}^{2}\left(\mathbb{R}^{2} \backslash \bar{B}_{\delta}(0)\right)} .
\end{aligned}
$$

Therefore, if $m \geq 3$, we have $(m+1)^{4}-4(m+1)^{3}=(m+1)^{3}(m-3) \geq 0$, so (10.11) implies that

$$
\begin{aligned}
\int_{\mathbb{R}^{2} \backslash \bar{B}_{\delta}(0)} \frac{(\nabla u \cdot x)^{2}}{|x|^{4}} d x & \leq \frac{1}{4(m+1)^{2}} \int_{\mathbb{R}^{2} \backslash \bar{B}_{\delta}(0)}\left(-2(m+1) \frac{x}{|x|^{2}} \cdot \nabla u+\frac{(m+1)^{2}}{|x|^{2}} u\right)^{2} d x \\
& =\frac{1}{4(m+1)^{2}} \int_{\mathbb{R}^{2} \backslash \bar{B}_{\delta}(0)}\left(\left(\mathscr{L}_{m}-\Delta\right) u\right)^{2} d x
\end{aligned}
$$


which implies by the triangle inequality that

$$
\left\|\frac{\nabla u \cdot x}{|x|^{2}}\right\|_{\mathrm{L}^{2}\left(\mathbb{R}^{2} \backslash \bar{B}_{\delta}(0)\right)} \leq \frac{1}{2(m+1)}\|\Delta u\|_{\mathrm{L}^{2}\left(\mathbb{R}^{2} \backslash \bar{B}_{\delta}(0)\right)}+\frac{1}{2(m+1)}\left\|\mathscr{L}_{m} u\right\|_{\mathrm{L}^{2}\left(\mathbb{R}^{2} \backslash \bar{B}_{\delta}(0)\right)} .
$$

If $1<m \leq 3$, then $(m+1)^{4}-4(m+1)^{3} \leq 0$, so we have by (10.5) and (10.5)

$$
\begin{aligned}
\int_{\mathbb{R}^{2} \backslash \bar{B}_{\delta}(0)}\left(\left(\mathscr{L}_{2}-\Delta\right) u\right)^{2} d x= & 4(m+1)^{2} \int_{\mathbb{R}^{2} \backslash \bar{B}_{\delta}(0)} \frac{(\nabla u \cdot x)^{2}}{|x|^{4}} d x \\
& +\left((m+1)^{4}-4(m+1)^{3}\right) \int_{\mathbb{R}^{2} \backslash \bar{B}_{\delta}(0)} \frac{u^{2}}{|x|^{4}} d x \\
\geq & \left((m+1)^{2}+(m+1)^{4}-4(m+1)^{3}\right) \int_{\mathbb{R}^{2} \backslash \bar{B}_{\delta}(0)} \frac{(\nabla u \cdot x)^{2}}{|x|^{4}} d x \\
= & (m+1)^{2}(m-1)^{2} \int_{\mathbb{R}^{2} \backslash \bar{B}_{\delta}(0)} \frac{(\nabla u \cdot x)^{2}}{|x|^{4}} d x,
\end{aligned}
$$

so that

$$
\begin{aligned}
\left\|\frac{\nabla u \cdot x}{|x|^{2}}\right\|_{\mathrm{L}^{2}\left(\mathbb{R}^{2} \backslash \bar{B}_{\delta}(0)\right)} \leq & \frac{1}{(m+1)(m-1)}\|\Delta u\|_{\mathrm{L}^{2}\left(\mathbb{R}^{2} \backslash \bar{B}_{\delta}(0)\right)} \\
& +\frac{1}{(m+1)(m-1)}\left\|\mathscr{L}_{m} u\right\|_{\mathrm{L}^{2}\left(\mathbb{R}^{2} \backslash \bar{B}_{\delta}(0)\right)} .
\end{aligned}
$$

This completes the proof of the theorem.

\subsection{Second variation for vectorial variations}

First, we prove a lemma giving the expression of the normal Jacobi operator of a smooth immersion acting on general variations.

Lemma 10.3 Let $\vec{\Phi}: \Sigma \rightarrow \mathbb{R}^{3}$ be a smooth immersion, and let $\mathscr{L}_{g}^{\perp}=\Delta_{g}^{\perp}+\mathscr{A}(\cdot)$ be the Jacobi operator of $\vec{\Phi}$, where $\mathscr{A}$ is the Simons operator. For all $\vec{v} \in C^{\infty}\left(\Sigma, \mathbb{R}^{3}\right)$, making the decomposition $\vec{v}=v \vec{n}_{\vec{\Phi}}+2 \operatorname{Re}(\alpha \otimes \partial \vec{\Phi})$, where $\alpha=f(z) \frac{1}{d z}$ is a $(-1,0)$ form, we have

$$
\mathscr{L}_{g}^{\perp} \vec{v}=\left(\mathscr{L}_{g} v+4 \operatorname{Re}(\alpha \otimes \partial H)\right) \vec{n},
$$

where $\mathscr{L}_{g}=\Delta+|A|^{2}$ and $A$ is the second fundamental form.

Proof Taking a complex chart, we recall that

$$
\begin{aligned}
\Delta_{g}^{\perp} & =4 e^{-2 \lambda} \operatorname{Re}\left(\nabla_{\partial_{z}}^{\perp} \nabla_{\partial_{\bar{z}}}^{\perp}\right) \\
\mathscr{A}(\vec{v}) & =-\frac{1}{2} \sum_{i, j=1}^{2}\left(\left\langle\nabla_{\partial_{x_{j}}} \vec{w}, \partial_{x_{i}} \vec{\Phi}\right\rangle+\left\langle\nabla_{\partial_{x_{i}}} \vec{w}, \partial_{x_{j}} \vec{\Phi}\right\rangle\right) \overrightarrow{\mathbb{I}}_{i, j} \\
& =-\langle d \vec{\Phi}, d \vec{w}\rangle_{g} \vec{H}-4 \operatorname{Re}\left(g^{-2} \otimes(\partial \vec{\Phi} \dot{\otimes} \partial \vec{w}) \otimes{\overrightarrow{h_{0}}}_{0}\right) .
\end{aligned}
$$

Since $\partial^{\perp} \vec{n}=0$, we immediately get

$$
\Delta_{g}^{\perp}(v \vec{n})=\left(\Delta_{g} v\right) \vec{n} \quad \text { and } \quad \mathscr{A}(v \vec{n})=\left(2 H^{2}+2\left|h_{0}\right|_{W P}^{2}\right) v \vec{n}=|A|^{2} v \vec{n} .
$$


Now, we have

$$
\begin{aligned}
\nabla_{\partial_{\bar{z}}} & \left(2 \operatorname{Re}\left(\alpha \partial_{z} \vec{\Phi}\right)\right)= \\
\nabla_{\partial_{z}}^{\perp} \nabla_{\partial_{\bar{z}}}^{\perp}\left(2 \operatorname{Re}\left(\alpha \partial_{z} \vec{\Phi}\right)\right)= & \frac{1}{2}\left(\partial_{\bar{z}} \alpha \partial_{z} \vec{\Phi}+\bar{\alpha} \nabla_{\partial_{\bar{z}}}^{\perp} \partial_{\bar{z}} \vec{\Phi}=\frac{1}{2} \alpha e^{2 \lambda} \vec{H}+\frac{1}{2} \bar{\alpha} \overline{\vec{h}}_{0}\right. \\
\Delta_{g}^{\perp}\left(2 \operatorname{Re}\left(\alpha \partial_{z} \vec{\Phi}\right)\right)= & 2 \operatorname{Re}\left(g^{-1} \otimes \partial(g \otimes \alpha) \vec{H}+\alpha \otimes e^{2 \lambda} \partial^{\perp} \vec{H}+\frac{1}{2} \partial_{z} \bar{\alpha} \overline{\vec{h}_{0}}+\frac{1}{2} \bar{\alpha} \overline{\bar{\partial}^{\perp}} \vec{h}_{0}\right. \\
& \left.+g^{-1} \otimes \bar{\partial} \alpha \otimes \vec{h}_{0}+g^{-1} \otimes \alpha \otimes \bar{\partial}^{\perp} \vec{h}_{0}\right)
\end{aligned}
$$

Since $\vec{\Phi}$ is conformal, we have $\left\langle\partial_{z} \vec{\Phi}, \partial_{z} \vec{\Phi}\right\rangle=\left\langle\partial_{z \bar{z}}^{2} \vec{\Phi}, \partial_{z} \vec{\Phi}\right\rangle=0$ and we deduce that

$$
\begin{aligned}
& \left\langle\partial_{z} \vec{\Phi}, \partial_{z}\left(2 \operatorname{Re}\left(\alpha \partial_{z} \vec{\Phi}\right)\right)\right\rangle=\partial_{z} \bar{\alpha}\left|\partial_{z} \vec{\Phi}\right|^{2}=\frac{1}{2} e^{2 \lambda} \partial_{z} \bar{\alpha} \\
& \left\langle\partial_{\bar{z}} \vec{\Phi}, \partial_{z}\left(2 \operatorname{Re}\left(\alpha \partial_{z} \vec{\Phi}\right)\right)\right\rangle=\frac{1}{2} e^{2 \lambda} \partial_{z} \alpha+\frac{1}{2} \alpha \partial_{z}\left(e^{2 \lambda}\right)=\frac{1}{2} \partial_{z}\left(e^{2 \lambda} \alpha\right) .
\end{aligned}
$$

Therefore, we deduce that

$$
\mathscr{A}(\vec{v}-v \vec{n})=-2 \operatorname{Re}\left(g^{-1} \otimes \partial(g \otimes \alpha) \vec{H}+g^{-1} \otimes \bar{\partial} \alpha \otimes \vec{h}_{0}\right) .
$$

Finally, we get

$$
\mathscr{L}_{g}^{\perp}(\vec{v}-v \vec{n})=2 \operatorname{Re}\left(\alpha \otimes \partial^{\perp} \vec{H}+g^{-1} \otimes \alpha \otimes \bar{\partial}^{\perp} \vec{h}_{0}\right)=4 \operatorname{Re}(\alpha \otimes \partial H)
$$

where we have used the Codazzi identity $\bar{\partial}^{\perp} \vec{h}_{0}=g \otimes \partial^{\perp} \vec{H}$. We deduce that

$$
\mathscr{L}_{g}^{\perp} \vec{v}=\left(\Delta_{g} v+|A|^{2} v+4 \operatorname{Re}(\alpha \otimes \partial H)\right) \vec{n}=\left(\mathscr{L}_{g} v+4 \operatorname{Re}(\alpha \otimes \partial H)\right) \vec{n} .
$$

In particular, if $\vec{\Phi}$ has constant mean curvature, the tangential part of $\vec{v}$ belongs to the Kernel of $\mathscr{L}_{g}^{\perp}$.

Proposition 10.4 Let $\vec{\Psi}: \Sigma \rightarrow \mathbb{R}^{3}$ be a smooth immersion. If $\vec{\Psi}_{t}=\vec{\Psi}+t \vec{w}$, we have

$$
\begin{aligned}
\frac{d^{2}}{d t^{2}}\left(K_{g_{t}} d \operatorname{vol}_{g_{t}}\right)_{\mid t=0}= & d \operatorname{Im}\left(2\left\langle\Delta_{g}^{\perp} \vec{w}-\mathscr{A}(\vec{w}), \partial^{\perp} \vec{w}\right\rangle-\partial\left|\nabla^{\perp} \vec{w}\right|_{g}^{2}\right. \\
& \left.+8 g^{-2} \otimes(\partial \vec{\Phi} \dot{\otimes} \bar{\partial} \vec{w}) \otimes\left(\vec{h}_{0} \dot{\otimes} \bar{\partial}^{\perp} \vec{w}\right)\right) .
\end{aligned}
$$

Proof First recall that by [28], we have for all $t \in(-\varepsilon, \varepsilon)$ (where $\varepsilon>0$ is a fixed real number small enough so that the variation $\vec{\Phi}_{t}=\vec{\Phi}+t \vec{w}+o(t)$ is an immersion for $t \in(-\varepsilon, \varepsilon)$ )

$$
\frac{d}{d t}\left(K_{g_{t}} d \operatorname{vol}_{g_{t}}\right)=d \operatorname{Im}\left(\frac{1}{\sqrt{\operatorname{det}\left(g_{t}\right)}}\left(4\left\langle\overrightarrow{\mathbb{I}}\left(\vec{e}_{z}, \vec{e}_{\bar{z}}\right), \nabla_{\vec{e}_{z}}^{\perp} \vec{w} d z\right\rangle-4\left\langle\overrightarrow{\mathbb{I}}\left(\vec{e}_{z}, \vec{e}_{z}\right), \nabla_{\vec{e}_{\bar{z}}}^{\perp} \vec{w} d z\right\rangle\right)\right) .
$$

In codimension 1, computations simplify significantly, and we have

$$
\frac{d}{d t}\left(K_{g_{t}} d \operatorname{vol}_{g_{t}}\right)=d \operatorname{Im}\left(\frac{1}{\sqrt{\operatorname{det}\left(g_{t}\right)}}\left(4\left\langle\partial_{z \bar{z}}^{2} \vec{\Phi}_{t}, \vec{n}_{t}\right\rangle\left\langle\partial_{\bar{z}} \vec{w}, \vec{n}_{t}\right\rangle d z-4\left\langle\partial_{z}^{2} \vec{\Phi}_{t}, \vec{n}_{t}\right\rangle\left\langle\partial_{z} \vec{w}, \vec{n}_{t}\right\rangle d z\right)\right) .
$$

Recalling that

$$
\left(\frac{d}{d t} \sqrt{\operatorname{det}\left(g_{t}\right)}\right)=\left\langle d \vec{\Phi}_{t}, d \vec{w}\right\rangle_{g_{t}} \sqrt{\operatorname{det}\left(g_{t}\right)}
$$


we deduce that

$$
\begin{aligned}
\frac{d^{2}}{d t^{2}} & \left(K_{g_{t}} d \operatorname{vol}_{g_{t}}\right)_{\mid t=0} \\
= & d \operatorname{Im}\left(-\langle d \vec{\Phi}, d \vec{w}\rangle_{g} e^{-2 \lambda}\left(4\left\langle\overrightarrow{\mathbb{I}}\left(\vec{e}_{z}, \vec{e}_{\bar{z}}\right), \nabla_{\vec{e}_{z}}^{\perp} \vec{w} d z\right\rangle-4\left\langle\overrightarrow{\mathbb{I}}\left(\vec{e}_{z}, \vec{e}_{z}\right), \nabla_{\vec{e}_{\bar{z}}}^{\perp} \vec{w} d z\right\rangle\right)\right. \\
& +e^{-2 \lambda}\left(4\left\langle\partial_{z \bar{z}}^{2} \vec{w}, \vec{n}\right\rangle\left\langle\partial_{z} \vec{w}, \vec{n}\right\rangle-4\left\langle\partial_{z}^{2} \vec{w}, \vec{n}\right\rangle\left\langle\partial_{\bar{z}} \vec{w}, \vec{n}\right\rangle-4\left\langle\partial_{z}^{2} \vec{\Phi}, \nabla_{\frac{d}{d t}} \vec{n}\right\rangle\left\langle\partial_{\bar{z}} \vec{w}, \vec{n}\right\rangle\right. \\
& \left.\left.-4\left\langle\partial_{z}^{2} \vec{\Phi}, \vec{n}\right\rangle\left\langle\partial_{\bar{z}} \vec{w}, \nabla_{\frac{d}{d t}} \vec{n}\right\rangle\right) d z\right) \\
= & d \operatorname{Im}\left(2\langle d \vec{\Phi}, d \vec{w}\rangle_{g} g^{-1} \otimes\left(\vec{h}_{0} \dot{\otimes} \bar{\partial}^{\perp} \vec{w}\right)+4 e^{-2 \lambda}((\mathrm{I})-(\mathrm{II})-(\mathrm{III})-(\mathrm{IV}))\right)
\end{aligned}
$$

First, we compute

$$
\begin{aligned}
e^{2 \lambda} \Delta_{g}^{\perp} \vec{w} & =2\left(\nabla_{\vec{e}_{\bar{z}}}^{\perp} \nabla_{\vec{e}_{\bar{z}}}^{\perp}+\nabla_{\vec{e}_{\bar{z}}}^{\perp} \nabla_{\vec{e}_{z}}^{\perp}\right) \vec{w}=2\left(\nabla_{\vec{e}_{\bar{z}}}^{\perp} \nabla_{\vec{e}_{\bar{z}}} \vec{w}+\nabla_{\vec{e}_{\bar{z}}}^{\perp} \nabla_{\vec{e}_{z}} \vec{w}\right)-2 \nabla_{\vec{e}_{\bar{z}}}^{\perp} \nabla_{\vec{e}_{\bar{z}}}^{\top} \vec{w}-2 \nabla_{\vec{e}_{\bar{z}}}^{\perp} \nabla_{\vec{e}_{\bar{z}}}^{\top} \vec{w} \\
& =e^{2 \lambda}\left(\Delta_{g} \vec{w}\right)^{\perp}-2 \nabla_{\vec{e}_{z}}^{\perp} \nabla_{\vec{e}_{\bar{z}}}^{\top} \vec{w}-2 \nabla_{\vec{e}_{\bar{z}}}^{\perp} \nabla_{\vec{e}_{\bar{z}}}^{\top} \vec{w}
\end{aligned}
$$

Now, we have

$$
\begin{aligned}
& \nabla_{\vec{e}_{z}}^{\top} \vec{w}=2 e^{-2 \lambda}\left\langle\partial_{z} \vec{w}, \vec{e}_{\bar{z}}\right\rangle \vec{e}_{z}+2 e^{-2 \lambda}\left\langle\partial_{z} \vec{w}, \vec{e}_{z}\right\rangle \vec{e}_{\bar{z}} \\
& \nabla_{\vec{e}_{\bar{z}}}^{\perp} \nabla_{\vec{e}_{z}}^{\top} \vec{w}=2 e^{-2 \lambda}\left\langle\partial_{z} \vec{w}, \vec{e}_{z}\right\rangle \overrightarrow{\mathbb{I}}\left(\vec{e}_{\bar{z}}, \vec{e}_{\bar{z}}\right)=g^{-1} \otimes(\partial \vec{\Phi} \dot{\otimes} \vec{w}) \otimes \overline{\vec{h}}_{0} \\
& \nabla_{\vec{e}_{z}}^{\perp} \nabla_{\vec{e}_{\bar{z}}}^{\top} \vec{w}=2 e^{-2 \lambda}\left\langle\partial_{\bar{z}} \vec{w}, \vec{e}_{\bar{z}}\right\rangle \mathbb{I}\left(\vec{e}_{\bar{z}}, \vec{e}_{\bar{z}}\right)=g^{-1} \otimes(\bar{\partial} \vec{\Phi} \dot{\otimes} \vec{\partial}) \otimes \vec{h}_{0} .
\end{aligned}
$$

Therefore, we have

$$
\Delta_{g}^{\perp} \vec{w}=\left(\Delta_{g} \vec{w}\right)^{\perp}-4 \operatorname{Re}\left(g^{-2} \otimes(\bar{\partial} \vec{\Phi} \dot{\otimes} \bar{\partial} \vec{w}) \otimes \vec{h}_{0}\right),
$$

and

$$
(\mathrm{I})=\left\langle\partial_{z \bar{z}}^{2} \vec{w}, \vec{n}\right\rangle\left\langle\partial_{z} \vec{w}, \vec{n}\right\rangle=\frac{1}{4} e^{2 \lambda}\left\langle\Delta_{g}^{\perp} \vec{w}+4 \operatorname{Re}\left(g^{-2} \otimes(\bar{\partial} \vec{\Phi} \dot{\otimes} \bar{\partial} \vec{w}) \otimes \vec{h}_{0}\right), \partial^{\perp} \vec{w}\right\rangle .
$$

Now, we have by the first line of (10.13)

$$
\begin{aligned}
(\mathrm{II}) & =\left\langle\partial_{z}^{2} \vec{w}, \vec{n}\right\rangle\left\langle\partial_{\bar{z}} \vec{w}, \vec{n}\right\rangle=\left\langle\nabla_{\vec{e}_{z}}^{\perp} \nabla_{\vec{e}_{z}} \vec{w}, \nabla_{\vec{e}_{\bar{z}}}^{\perp} \vec{w}\right\rangle=\left\langle\nabla_{\vec{e}_{z}}^{\perp} \nabla_{\vec{e}_{z}}^{\perp} \vec{w}, \nabla_{\vec{e}_{\bar{z}}}^{\perp} \vec{w}\right\rangle+\left\langle\nabla_{\vec{e}_{z}}^{\perp} \nabla_{\vec{e}_{z}}^{\top} \vec{w}, \nabla_{\vec{e}_{\bar{z}}}^{\perp} \vec{w}\right\rangle \\
& =\left\langle\nabla_{\vec{e}_{z}}^{\perp} \nabla_{\vec{e}_{z}}^{\perp} \vec{w}, \nabla_{\vec{e}_{\bar{z}}}^{\perp} \vec{w}\right\rangle+g^{-1} \otimes(\bar{\partial} \vec{\Phi} \dot{\otimes} \partial \vec{w}) \otimes\left(\vec{h}_{0} \dot{\otimes} \bar{\partial}^{\perp} \vec{w}\right) \\
& =\partial\left|\partial^{\perp} \vec{w}\right|^{2}-\left\langle\nabla_{\vec{e}_{z}}^{\perp} \vec{w}, \nabla_{\vec{e}_{z}}^{\perp} \nabla_{\vec{e}_{\bar{z}}}^{\perp} \vec{w}\right\rangle+g^{-1} \otimes(\bar{\partial} \vec{\Phi} \dot{\otimes} \partial \vec{w}) \otimes\left(\vec{h}_{0} \dot{\otimes} \bar{\partial}^{\perp} \vec{w}\right)
\end{aligned}
$$

Notice now that by (10.13), we have

$$
\begin{aligned}
\nabla_{\vec{e}_{z}}^{\perp} \nabla_{\vec{e}_{\bar{z}}}^{\perp} \vec{w} & =\nabla_{\vec{e}_{z}}^{\perp} \nabla_{\vec{e}_{\bar{z}}} \vec{w}-\nabla_{\vec{e}_{z}}^{\perp} \nabla_{\vec{e}_{\bar{z}}}^{\top} \vec{w}=\nabla_{\vec{e}_{\bar{z}}}^{\perp} \nabla_{\vec{e}_{z}} \vec{w}-\nabla_{\vec{e}_{z}}^{\perp} \nabla_{\vec{e}_{\bar{z}}}^{\top} \vec{w} \\
& =\nabla_{\vec{e}_{\bar{z}}}^{\perp} \nabla_{\vec{e}_{z}}^{\perp} \vec{w}+\nabla_{\vec{e}_{\bar{z}}}^{\perp} \nabla_{\vec{e}_{z}}^{\top} \vec{w}-\nabla_{\vec{e}_{z}}^{\perp} \nabla_{\vec{e}_{\bar{z}}}^{\top} \vec{w} \\
& =\nabla_{\vec{e}_{\bar{z}}}^{\perp} \nabla_{\vec{e}_{z}}^{\perp} \vec{w}+g^{-1} \otimes\left((\partial \vec{\Phi} \dot{\otimes} \partial \vec{w}) \otimes{\overrightarrow{h_{0}}}_{0}-(\bar{\partial} \vec{\Phi} \dot{\otimes} \bar{\partial} \vec{w}) \otimes \vec{h}_{0}\right) .
\end{aligned}
$$

Therefore, we have

$\left\langle\nabla_{\vec{e}_{z}}^{\perp} \vec{w}, \nabla_{\vec{e}_{z}}^{\perp} \nabla_{\vec{e}_{\bar{z}}}^{\perp} \vec{w}\right\rangle$ 


$$
\begin{aligned}
& =\left\langle\frac{1}{2}\left(\nabla_{\vec{e}_{z}}^{\perp} \nabla_{\vec{e}_{\bar{z}}}^{\perp}+\nabla_{\vec{e}_{\bar{z}}}^{\perp} \nabla_{\vec{e}_{z}}^{\perp}\right) \vec{w}+\frac{1}{2} g^{-1} \otimes\left((\partial \vec{\Phi} \dot{\otimes} \partial \vec{w}) \otimes \overrightarrow{\vec{h}_{0}}-(\bar{\partial} \vec{\Phi} \dot{\otimes} \vec{\partial}) \otimes \vec{h}_{0}\right), \nabla_{\vec{e}_{z}}^{\perp} \vec{w}\right\rangle \\
& =\frac{1}{4} e^{2 \lambda}\left\langle\Delta_{g}^{\perp} \vec{w}, \partial^{\perp} \vec{w}\right\rangle+\frac{1}{2} g^{-1} \otimes(\partial \vec{\Phi} \dot{\otimes} \partial \vec{w}) \otimes\left(\vec{h}_{0} \dot{\otimes} \partial^{\perp} \vec{w}\right) \\
& -\frac{1}{2} g^{-1}(\bar{\partial} \vec{\Phi} \dot{\otimes} \bar{\partial} \vec{w}) \otimes\left(\vec{h}_{0} \dot{\otimes} \partial^{\perp} \vec{w}\right) .
\end{aligned}
$$

Finally, we get by (10.15), (10.16) and (10.17)

$$
\begin{aligned}
(\mathrm{II})= & -\frac{1}{4} e^{2 \lambda}\left\langle\Delta_{g}^{\perp} \vec{w}, \partial^{\perp} \vec{w}\right\rangle+\partial\left|\partial^{\perp} \vec{w}\right|^{2}+g^{-1} \otimes(\bar{\partial} \vec{\Phi} \dot{\otimes} \vec{w}) \otimes\left(\vec{h}_{0} \dot{\otimes} \bar{\partial}^{\perp} \vec{w}\right) \\
& +\frac{1}{2} g^{-1}(\bar{\partial} \vec{\Phi} \dot{\otimes} \bar{\partial} \vec{w}) \otimes\left(\vec{h}_{0} \dot{\otimes} \partial^{\perp} \vec{w}\right)-\frac{1}{2} g^{-1} \otimes(\partial \vec{\Phi} \dot{\otimes} \vec{w}) \otimes\left(\vec{h}_{0} \dot{\otimes} \partial^{\perp} \vec{w}\right) .
\end{aligned}
$$

Since $\left|\vec{n}_{t}\right|^{2}=1$, we have $\left\langle\nabla_{\frac{d}{d t}} \vec{n}_{t}, \vec{n}_{t}\right\rangle=0$, which implies that

$$
\left(\nabla_{\frac{d}{d t}} \vec{n}_{t}\right)_{\mid t=0}=-2 e^{-2 \lambda}\left\langle\vec{n}, \partial_{\bar{z}} \vec{w}\right\rangle \vec{e}_{z}-2 e^{-2 \lambda}\left\langle\vec{n}, \partial_{z} \vec{w}\right\rangle \vec{e}_{\bar{z}}
$$

Therefore, we have as $\left\langle\vec{e}_{z}, \vec{e}_{z}\right\rangle=0,\left|\vec{e}_{z}\right|^{2}=\frac{1}{2} e^{2 \lambda}$, and $\left(\partial_{z \bar{z}}^{2} \vec{\Phi}\right)^{\top}=0$

$$
\begin{aligned}
\text { (III) } & =\left\langle\partial_{z}^{2} \vec{\Phi}, \nabla_{\frac{d}{d t}} \vec{n}\right\rangle\left\langle\partial_{\bar{z}} \vec{w}, \vec{n}\right\rangle=\left\langle\nabla_{\vec{e}_{z}} \vec{e}_{z},-2 e^{-2 \lambda}\left\langle\partial_{\bar{z}} \vec{w}, \vec{n}\right\rangle \vec{e}_{z}-2 e^{-2 \lambda}\left\langle\partial_{z} \vec{w}, \vec{n}\right\rangle \vec{e}_{\bar{z}}\right\rangle\left\langle\partial_{\bar{z}} \vec{w}, \vec{n}\right\rangle \\
& =-2\left\langle\partial_{z}^{2} \vec{\Phi}, \partial_{\bar{z}} \vec{\Phi}\right\rangle\left\langle\nabla_{\vec{e}_{z}}^{\perp} \vec{w}, \nabla_{\vec{e}_{\bar{z}}}^{\perp} \vec{w}\right\rangle=-e^{-2 \lambda} \partial_{z}\left(e^{2 \lambda}\right)\left|\partial^{\perp} \vec{w}\right|^{2}=e^{2 \lambda} \partial_{z}\left(e^{-2 \lambda}\right)\left|\partial^{\perp} \vec{w}\right|^{2} .
\end{aligned}
$$

Finally, we have

$$
\begin{aligned}
(\mathrm{IV})= & \left\langle\partial_{z}^{2} \vec{\Phi}, \vec{n}\right\rangle\left\langle\partial_{\bar{z}} \vec{w}, \nabla_{\frac{d}{d t}} \vec{n}\right\rangle=\frac{1}{2} h_{0}\left\langle\partial_{\bar{z}} \vec{w},-2 e^{-2 \lambda}\left\langle\vec{n}, \partial_{\bar{z}} \vec{w}\right\rangle \vec{e}_{z}-2 e^{-2 \lambda}\left\langle\vec{n}, \partial_{z} \vec{w}\right\rangle \vec{e}_{\bar{z}}\right\rangle \\
& =-g^{-1} \otimes(\partial \vec{\Phi} \dot{\otimes} \bar{\partial} \vec{w}) \otimes\left(\vec{h}_{0} \dot{\otimes} \bar{\partial}^{\perp} \vec{w}\right)-g^{-1} \otimes(\bar{\partial} \vec{\Phi} \dot{\otimes} \bar{\partial} \vec{w}) \otimes\left(\vec{h}_{0} \dot{\otimes} \partial^{\perp} \vec{w}\right) .
\end{aligned}
$$

Since

$$
\partial\left|\partial^{\perp} \vec{w}\right|^{2}+e^{2 \lambda} \partial_{z}\left(e^{-2 \lambda}\right)\left|\partial^{\perp} \vec{w}\right|^{2}=e^{2 \lambda} \partial_{z}\left(e^{-2 \lambda}\left|\partial^{\perp} \vec{w}\right|^{2}\right)=e^{2 \lambda} \partial\left|\partial^{\perp} \vec{w}\right|_{g}^{2},
$$

we deduce by (10.18), (10.19) and (10.20) that

$$
\begin{aligned}
(\mathrm{II})+(\mathrm{III})+(\mathrm{IV})= & -\frac{1}{4} e^{2 \lambda}\left\langle\Delta_{g}^{\perp} \vec{w}, \partial^{\perp} \vec{w}\right\rangle+e^{2 \lambda} \partial\left|\partial^{\perp} \vec{w}\right|_{g}^{2} \\
& +g^{-1} \otimes((\bar{\partial} \vec{\Phi} \dot{\otimes} \partial \vec{w})-(\partial \vec{\Phi} \dot{\otimes} \bar{\partial} \vec{w})) \otimes\left(\vec{h}_{0} \dot{\otimes} \bar{\partial}^{\perp} \vec{w}\right) \\
& -\frac{1}{2} g^{-1}(\bar{\partial} \vec{\Phi} \dot{\otimes} \bar{\partial} \vec{w}) \otimes\left(\vec{h}_{0} \dot{\otimes} \partial^{\perp} \vec{w}\right) \\
& -\frac{1}{2} g^{-1} \otimes(\partial \vec{\Phi} \dot{\otimes} \vec{w}) \otimes\left(\overline{\vec{h}}_{0} \dot{\otimes} \partial^{\perp} \vec{w}\right) \\
= & -\frac{1}{4} e^{2 \lambda}\left\langle\Delta_{g}^{\perp} \vec{w}, \partial^{\perp} \vec{w}\right\rangle+e^{2 \lambda} \partial\left|\partial^{\perp} \vec{w}\right|_{g}^{2} \\
& +g^{-1} \otimes((\bar{\partial} \vec{\Phi} \dot{\otimes} \partial \vec{w})-(\partial \vec{\Phi} \dot{\otimes} \bar{\partial} \vec{w})) \otimes\left(\vec{h}_{0} \dot{\otimes} \bar{\partial}^{\perp} \vec{w}\right) \\
& -\left\langle\operatorname{Re}\left(g^{-1} \otimes(\bar{\partial} \vec{\Phi} \dot{\otimes} \vec{\partial} \vec{w}) \otimes \vec{h}_{0}\right), \partial^{\perp} \vec{w}\right\rangle
\end{aligned}
$$


This implies that

$$
\begin{aligned}
\text { (I) }- & (\mathrm{II})-(\mathrm{III})-(\mathrm{IV}) \\
= & \frac{1}{2} e^{2 \lambda}\left\langle\Delta_{g}^{\perp} \vec{w}+4 \operatorname{Re}\left(g^{-2} \otimes(\bar{\partial} \vec{\Phi} \dot{\otimes} \bar{\partial} \vec{w}) \otimes \vec{h}_{0}\right), \partial^{\perp} \vec{w}\right\rangle \\
& -e^{2 \lambda} \partial\left|\partial^{\perp} \vec{w}\right|_{g}^{2}+g^{-1} \otimes((\partial \vec{\Phi} \dot{\otimes} \bar{\partial} \vec{w})-(\bar{\partial} \vec{\Phi} \dot{\otimes} \partial \vec{w})) \otimes\left(\vec{h}_{0} \dot{\otimes} \bar{\partial}^{\perp} \vec{w}\right)
\end{aligned}
$$

and

$$
\begin{aligned}
4 e^{2 \lambda} & ((\mathrm{I})-(\mathrm{II})-(\mathrm{III})-(\mathrm{IV})) \\
= & 2\left\langle\Delta_{g}^{\perp} \vec{w}+4 \operatorname{Re}\left(g^{-2} \otimes(\bar{\partial} \vec{\Phi} \dot{\otimes} \bar{\partial} \vec{w}) \otimes \vec{h}_{0}\right), \partial^{\perp} \vec{w}\right\rangle-4 \partial\left|\partial^{\perp} \vec{w}\right|_{g}^{2} \\
& +4 g^{-2} \otimes((\partial \vec{\Phi} \dot{\otimes} \bar{\partial} \vec{w})-(\bar{\partial} \vec{\Phi} \dot{\otimes} \partial \vec{w})) \otimes\left(\vec{h}_{0} \dot{\otimes} \bar{\partial}^{\perp} \vec{w}\right) .
\end{aligned}
$$

Since $\langle d \vec{\Phi}, d \vec{w}\rangle_{g}=4 \operatorname{Re}\left(g^{-1} \otimes(\partial \vec{\Phi} \dot{\otimes} \bar{\partial} \vec{w})\right)$, we deduce that

$$
\begin{aligned}
2\langle d \vec{\Phi}, d \vec{w}\rangle_{g} g^{-1} \otimes\left(\vec{h}_{0} \dot{\otimes} \bar{\partial}^{\perp} \vec{w}\right)+4 g^{-2} \otimes((\partial \vec{\Phi} \dot{\otimes} \bar{\partial} \vec{w})-(\bar{\partial} \vec{\Phi} \dot{\otimes} \partial \vec{w})) \otimes\left(\vec{h}_{0} \dot{\otimes} \bar{\partial}^{\perp} \vec{w}\right) \\
=4 g^{-2} \otimes(\partial \vec{\Phi} \dot{\otimes} \bar{\partial} \vec{w}+\bar{\partial} \vec{\Phi} \dot{\otimes} \partial \vec{w}) \otimes\left(\vec{h}_{0} \dot{\otimes} \bar{\partial}^{\perp}\right) \\
\quad+4 g^{-2} \otimes((\partial \vec{\Phi} \dot{\otimes} \bar{\partial} \vec{w})-(\bar{\partial} \vec{\Phi} \dot{\otimes} \partial \vec{w})) \otimes\left(\vec{h}_{0} \dot{\otimes} \bar{\partial}^{\perp} \vec{w}\right) \\
=8 g^{-2} \otimes(\partial \vec{\Phi} \dot{\otimes} \bar{\partial} \vec{w}) \otimes\left(\vec{h}_{0} \dot{\otimes} \bar{\partial}^{\perp} \vec{w}\right) .
\end{aligned}
$$

Finally, we deduce that

$$
\begin{aligned}
\frac{d^{2}}{d t^{2}}\left(K_{g_{t}} d \operatorname{vol}_{g_{t}}\right)_{\mid t=0}= & d \operatorname{Im}\left(2\left\langle\Delta_{g}^{\perp} \vec{w}+4 \operatorname{Re}\left(g^{-2} \otimes(\bar{\partial} \vec{\Phi} \dot{\otimes} \vec{\partial}) \otimes \vec{h}_{0}\right), \partial^{\perp} \vec{w}\right\rangle-4 \partial\left|\partial^{\perp} \vec{w}\right|_{g}^{2}\right. \\
& \left.+8 g^{-2} \otimes(\partial \vec{\Phi} \dot{\otimes} \bar{\partial} \vec{w}) \otimes\left(\vec{h}_{0} \dot{\otimes} \bar{\partial}^{\perp} \vec{w}\right)\right) \\
= & d \operatorname{Im}\left(2\left\langle\Delta_{g}^{\perp} \vec{w}-\mathscr{A}(\vec{w}), \partial^{\perp} \vec{w}\right\rangle-\partial\left|\nabla^{\perp} \vec{w}\right|_{g}^{2}\right. \\
& \left.+8 g^{-2} \otimes(\partial \vec{\Phi} \dot{\otimes} \bar{\partial} \vec{w}) \otimes\left(\vec{h}_{0} \dot{\otimes} \bar{\partial}^{\perp} \vec{w}\right)\right),
\end{aligned}
$$

where $\mathscr{A}$ is the Simons operator.

We now recall Theorem 3.3. Here $\mathscr{W}$ is the conformal Willmore energy, defined by

$$
\mathscr{W}(\vec{\Phi})=\int_{\Sigma}\left(|\vec{H}|^{2}-K_{g}\right) d \operatorname{vol}_{g}
$$

Theorem 10.5 Let $\vec{\Psi}: \Sigma \rightarrow \mathbb{R}^{3}$ be a branched Willmore sphere, and assume that $\vec{\Psi}$ is the inversion of a complete minimal surface $\vec{\Phi}: \Sigma \backslash\left\{p_{1}, \ldots, p_{n}\right\} \rightarrow \mathbb{R}^{3}$ with finite total curvature, and let $\vec{v}$ be an admissible variation. Make the decomposition $\vec{v}=-v \vec{n}_{\vec{\Psi}}+$ $2 \operatorname{Re}(\alpha \otimes \partial \vec{\Psi})$. If $u=|\vec{\Phi}|^{2} v$, we have

$$
\begin{aligned}
& D^{2} \mathscr{W}(\vec{\Psi})(\vec{v}, \vec{v})=\int_{\Sigma}\left(\frac{1}{2}\left(\mathscr{L}_{g} u\right)^{2} d \mathrm{vol}_{g}-d \operatorname{Im}\left(\left(\Delta_{g} u+2 K_{g} u+4 \operatorname{Re}\left(g^{-1} \otimes h_{0} \otimes \bar{\partial} \alpha\right)\right)\left(2 \partial u+h_{0} \otimes \alpha\right)\right.\right. \\
& -\partial\left|2 \partial u+h_{0} \otimes \alpha\right|_{g}^{2}+2 g^{-1} \otimes \bar{\partial}(g \otimes \bar{\alpha}) \otimes\left(2 g^{-1} \otimes h_{0} \otimes \bar{\partial} u-K_{g} g \otimes \bar{\alpha}\right)
\end{aligned}
$$




$$
\begin{aligned}
& +4\left\langle\vec{\Phi}, \vec{n}_{\vec{\Phi}}\right\rangle g^{-1} \otimes h_{0} \otimes \bar{\partial}\left(|\vec{\Phi}|^{2} v^{2}+\frac{1}{|\vec{\Phi}|^{2}} g \otimes|\alpha|^{2}\right) \\
& -8 g^{-1} \otimes h_{0} \otimes \bar{\partial}\left(|\vec{\Phi}|^{2}\left\langle\vec{\Phi}, \vec{n}_{\vec{\Phi}}\right\rangle v^{2}+2 \operatorname{Re}(\alpha \otimes \partial \log |\vec{\Phi}|)|\vec{\Phi}|^{2} v\right) \\
& \left.\left.+4 K_{g}\left(\left\langle\vec{\Phi}, \vec{n}_{\vec{\Phi}}\right\rangle v+2 \operatorname{Re}(\alpha \otimes \partial \log |\vec{\Phi}|)\right) g \otimes \bar{\alpha}\right)\right)=\int_{\Sigma}\left(\frac{1}{2}\left(\mathscr{L}_{g} u\right)^{2} d \operatorname{vol}_{g}-d \omega(u, \alpha)\right),
\end{aligned}
$$

where $g=\vec{\Phi}^{*} g_{\mathbb{R}^{3}}$, and $\mathscr{L}_{g}=\Delta_{g}-2 K_{g}$ is the Jacobi operator of $\vec{\Phi}$.

Proof Now, if we consider a variation such that $\frac{d^{2}}{d t^{2}}\left(\vec{\Phi}_{t}\right)_{\mid t=0}=\vec{u}_{2} \neq 0$, we have since $\vec{H}=0$ by the previous Proposition 10.4

$$
\begin{aligned}
& \frac{d^{2}}{d t^{2}}\left(K_{g_{t}} d \operatorname{vol}_{g_{t}}\right)_{t=0}=d \operatorname{Im}\left(2\left\langle\Delta_{g}^{\perp} \mathscr{A}(\vec{u}), \partial^{\perp} \vec{u}\right\rangle-\partial\left|\nabla^{\perp} \vec{u}\right|_{g}^{2}\right. \\
& \left.\quad+8 g^{-2} \otimes(\partial \vec{\Phi} \dot{\otimes} \bar{\partial} \vec{u}) \otimes\left(\vec{h}_{0} \dot{\otimes} \bar{\partial}^{\perp} \vec{u}\right)\right) \\
& \quad+d \operatorname{Im}\left(\frac{1}{\sqrt{\operatorname{det}(g)}}\left(\left\langle\mathbb{I}\left(\vec{e}_{z}, \vec{e}_{\bar{z}}\right), \nabla_{\vec{e}_{z}}^{\perp} \vec{u}_{2} d z\right\rangle-4\left\langle\overrightarrow{\mathbb{I}}\left(\vec{e}_{z}, \vec{e}_{z}\right), \nabla_{\vec{e}_{\bar{z}}}^{\perp} \vec{u}_{2}\right\rangle d z\right)\right) \\
& =d \operatorname{Im}\left(2\left\langle\Delta_{g}^{\perp} \vec{u}-\mathscr{A}(\vec{u}), \partial^{\perp} \vec{u}\right\rangle-\partial\left|\nabla^{\perp} \vec{u}\right|_{g}^{2}\right. \\
& \left.\quad+8 g^{-2} \otimes(\partial \vec{\Phi} \dot{\otimes} \vec{\partial} \vec{u}) \otimes\left(\vec{h}_{0} \dot{\otimes} \bar{\partial}^{\perp} \vec{u}\right)-2 g^{-1} \otimes\left(\vec{h}_{0} \dot{\otimes} \bar{\partial}^{\perp} \vec{u}_{2}\right)\right) .
\end{aligned}
$$

If $\vec{\Psi}=\frac{\vec{\Phi}}{|\vec{\Phi}|^{2}}: \Sigma \rightarrow \mathbb{R}^{3}$, and we consider a variation $\vec{\Psi}+t \vec{v}$, we have

$$
\begin{aligned}
\vec{\Phi}_{t} & =\frac{\vec{\Psi}_{t}}{\left|\vec{\Psi}_{t}\right|^{2}}=\frac{\vec{\Psi}+t \vec{v}}{|\vec{\Psi}|^{2}+2 t\langle\vec{\Psi}, \vec{v}\rangle+t^{2}|\vec{v}|^{2}}=\frac{\vec{\Phi}+t|\vec{\Phi}|^{2} \vec{v}}{1+2 t\langle\vec{\Phi}, \vec{v}\rangle+t^{2}|\vec{\Phi}|^{2}|\vec{v}|^{2}} \\
& =\vec{\Phi}+t\left(|\vec{\Phi}|^{2} \vec{v}-2\langle\vec{\Phi}, \vec{v}\rangle \vec{\Phi}\right)+t^{2}\left(-|\vec{\Phi}|^{2}|\vec{v}|^{2} \vec{\Phi}\right. \\
& \left.-2\langle\vec{\Phi}, \vec{v}\rangle\left(|\vec{\Phi}|^{2} \vec{v}-2\langle\vec{\Phi}, \vec{v}\rangle \vec{\Phi}\right)\right)
\end{aligned}
$$

Therefore, we have

$$
\begin{aligned}
\vec{u} & =\frac{d}{d t}\left(\vec{\Phi}_{t}\right)_{\mid t=0}=|\vec{\Phi}|^{2} \vec{v}-2\langle\vec{\Phi}, \vec{v}\rangle \vec{\Phi} \\
\vec{u}_{2} & =\frac{d^{2}}{d t^{2}}\left(\vec{\Phi}_{t}\right)_{\mid t=0}=-2|\vec{\Phi}|^{2}|\vec{v}|^{2} \vec{\Phi}-4\langle\vec{\Phi}, \vec{v}\rangle\left(|\vec{\Phi}|^{2} \vec{v}-2\langle\vec{\Phi}, \vec{v}\rangle \vec{\Phi}\right) .
\end{aligned}
$$

Now, if for some function $v: \Sigma \rightarrow \mathbb{R}$, a $(-1,0)$-form $\alpha: \Sigma \rightarrow \mathbb{C}$, we have

$$
\vec{v}=-v \vec{n}_{\vec{\Psi}}+2 \operatorname{Re}(\alpha \otimes \partial \vec{\Psi}) \text {. }
$$

By Lemma 10.7, we deduce that

$$
\begin{aligned}
\vec{u} & =|\vec{\Phi}|^{2} \vec{v}-2\langle\vec{\Phi}, \vec{v}\rangle \vec{\Phi} \\
& =-|\vec{\Phi}|^{2} v \vec{n}_{\vec{\Psi}}+2|\vec{\Phi}|^{2} \operatorname{Re}\left(\alpha \partial_{z} \vec{\Psi}\right)-2\left\langle\vec{\Phi},-v \vec{n}_{\vec{\Psi}}+2 \operatorname{Re}\left(\alpha \partial_{z} \vec{\Psi}\right)\right\rangle \vec{\Phi} \\
& =-|\vec{\Phi}|^{2} v\left(\vec{n}_{\vec{\Psi}}-2\left\langle\vec{\Phi}, \vec{n}_{\vec{\Psi}}\right\rangle \frac{\vec{\Phi}}{|\vec{\Phi}|^{2}}\right)+2|\vec{\Phi}|^{2} \operatorname{Re}\left(\alpha \partial_{z} \vec{\Psi}\right)-4\left\langle\vec{\Phi}, \operatorname{Re}\left(\alpha \partial_{z} \vec{\Psi}\right)\right\rangle \vec{\Phi}
\end{aligned}
$$




$$
=|\vec{\Phi}|^{2} v \vec{n}_{\vec{\Phi}}+2 \operatorname{Re}(\alpha \otimes \partial \vec{\Phi}),
$$

where we have use

$$
\begin{aligned}
\left\langle\vec{\Phi}, \partial_{z} \vec{\Psi}\right\rangle & =\left\langle\vec{\Phi}, \frac{\partial_{z} \vec{\Phi}}{|\vec{\Phi}|^{2}}-2 \frac{\left\langle\partial_{z} \vec{\Phi}, \vec{\Phi}\right\rangle}{|\vec{\Phi}|^{4}} \vec{\Phi}\right\rangle=-\frac{\left\langle\partial_{z} \vec{\Phi}, \vec{\Phi}\right\rangle}{|\vec{\Phi}|^{2}} \\
|\vec{\Phi}|^{2} \partial_{z} \vec{\Psi}-2\left\langle\vec{\Phi}, \partial_{z} \vec{\Psi}\right\rangle \vec{\Phi} & =|\vec{\Phi}|^{2}\left(\frac{\partial_{z} \vec{\Phi}}{|\vec{\Phi}|^{2}}-2 \frac{\left\langle\partial_{z} \vec{\Phi}, \vec{\Phi}\right\rangle}{|\vec{\Phi}|^{4}} \vec{\Phi}\right)+2 \frac{\left\langle\partial_{z} \vec{\Phi}, \vec{\Phi}\right\rangle}{|\vec{\Phi}|^{2}} \vec{\Phi}=\partial_{z} \vec{\Phi}
\end{aligned}
$$

Finally, we deduce that

$$
\vec{u}=\left(|\vec{\Phi}|^{2} v\right) \vec{n}_{\vec{\Phi}}+2 \operatorname{Re}(\alpha \otimes \partial \vec{\Phi})=u \vec{n}_{\vec{\Phi}}+2 \operatorname{Re}(\alpha \otimes \partial \vec{\Phi}),
$$

where $u=|\vec{\Phi}|^{2} v$. Notice that we have

$$
\left|\partial_{z} \vec{\Psi}\right|^{2}=\left|\frac{\partial_{z} \vec{\Phi}}{|\vec{\Phi}|^{2}}-2 \frac{\left\langle\partial_{z} \vec{\Phi}, \vec{\Phi}\right\rangle}{|\vec{\Phi}|^{4}} \vec{\Phi}\right|^{2}=\frac{\left|\partial_{z} \vec{\Phi}\right|^{2}}{|\vec{\Phi}|^{4}}
$$

Therefore, we deduce that

$$
|\vec{v}|^{2}=v^{2}+2\left|\partial_{z} \vec{\Psi}\right|^{2}|\alpha|^{2}=v^{2}+\frac{1}{|\vec{\Phi}|^{4}} g_{\vec{\Phi}} \otimes|\alpha|^{2} .
$$

This implies that

$$
|\vec{\Phi}|^{2}|\vec{v}|^{2} \vec{\Phi}=|\vec{\Phi}|^{2} v^{2} \vec{\Phi}+g_{\vec{\Phi}} \otimes|\alpha|^{2} \frac{\vec{\Phi}}{|\vec{\Phi}|^{2}}=\left(u^{2}+g_{\vec{\Phi}} \otimes|\alpha|^{2}\right) \frac{\vec{\Phi}}{|\vec{\Phi}|^{2}}
$$

A direct computation shows that

$$
\left\langle\vec{\Phi}, \vec{n}_{\vec{\Psi}}\right\rangle=\left\langle\vec{\Phi},-\vec{n}_{\vec{\Phi}}+2\left\langle\vec{\Phi}, \vec{n}_{\vec{\Phi}}\right\rangle \frac{\vec{\Phi}}{|\vec{\Phi}|^{2}}\right\rangle=\left\langle\vec{\Phi}, \vec{n}_{\vec{\Phi}}\right\rangle
$$

and

$$
\langle\vec{\Phi}, \vec{v}\rangle=-\left\langle\vec{\Phi}, \vec{n}_{\Psi}\right\rangle v-2 \operatorname{Re}\left(\alpha \frac{\left\langle\partial_{z} \vec{\Phi}, \vec{\Phi}\right\rangle}{|\vec{\Phi}|^{2}}\right)=-\left\langle\vec{\Phi}, \vec{n}_{\vec{\Phi}}\right\rangle v-2 \operatorname{Re}\left(\alpha \frac{\left\langle\partial_{z} \vec{\Phi}, \vec{\Phi}\right\rangle}{|\vec{\Phi}|^{2}}\right)
$$

Therefore, we have

$$
-\langle\vec{\Phi}, \vec{v}\rangle\left(|\vec{\Phi}|^{2} \vec{v}-2\langle\vec{\Phi}, \vec{v}\rangle \vec{\Phi}\right)=\left(v\left\langle\vec{\Phi}, \vec{n}_{\vec{\Phi}}\right\rangle+2 \operatorname{Re}\left(\alpha \frac{\left\langle\partial_{z} \vec{\Phi}, \vec{\Phi}\right\rangle}{|\vec{\Phi}|^{2}}\right)\right)\left(u \vec{n}_{\vec{\Phi}}+2 \operatorname{Re}\left(\alpha \partial_{z} \vec{\Phi}\right)\right) .
$$

Therefore, we have by (10.23), (10.24) and (10.25)

$$
\begin{aligned}
\vec{u}_{2}= & -2\left(u^{2}+g \otimes|\alpha|^{2}\right) \frac{\vec{\Phi}}{|\vec{\Phi}|^{2}}+\left(\left\langle\vec{\Phi}, \vec{n}_{\vec{\Phi}}\right\rangle v\right. \\
& \left.+2 \operatorname{Re}\left(\alpha \frac{\left\langle\partial_{z} \vec{\Phi}, \vec{\Phi}\right\rangle}{|\vec{\Phi}|^{2}}\right)\right)\left(4 u \vec{n}_{\vec{\Phi}}+8 \operatorname{Re}(\alpha \otimes \partial \vec{\Phi})\right) \\
= & -2\left(u^{2}+g \otimes|\alpha|^{2}\right) \frac{\vec{\Phi}}{|\vec{\Phi}|^{2}}
\end{aligned}
$$




$$
+\left(\left\langle\vec{\Phi}, \vec{n}_{\vec{\Phi}}\right\rangle v+2 \operatorname{Re}(\alpha \otimes \partial \log |\vec{\Phi}|)\right)\left(4 u \vec{n}_{\vec{\Phi}}+8 \operatorname{Re}(\alpha \otimes \partial \vec{\Phi})\right) .
$$

By (10.12), since $\vec{H}=0$, we get

$$
\begin{aligned}
\bar{\partial}^{\perp} \vec{u}_{2}= & -2 \bar{\partial}\left(|\vec{\Phi}|^{2} v^{2}+\frac{1}{|\vec{\Phi}|^{2}} g \otimes|\alpha|^{2}\right) \vec{\Phi} \\
& +4 \bar{\partial}\left(|\vec{\Phi}|^{2}\left\langle\vec{\Phi}, \vec{n}_{\vec{\Phi}}\right\rangle v^{2}+2 \operatorname{Re}(\alpha \otimes \partial \log |\vec{\Phi}|) v\right) \vec{n}_{\vec{\Phi}} \\
& +\left(\left\langle\vec{\Phi}, \vec{n}_{\vec{\Phi}}\right\rangle v+2 \operatorname{Re}(\alpha \otimes \partial \log |\vec{\Phi}|)\right) 2 \bar{\alpha} \otimes \overline{\vec{h}}_{0}
\end{aligned}
$$

Finally, we have

$$
\begin{aligned}
& -2 g^{-1} \otimes\left(\vec{h}_{0} \dot{\otimes} \bar{\partial}^{\perp} \vec{u}_{2}\right)=4\left\langle\vec{\Phi}, \vec{n}_{\vec{\Phi}}\right\rangle g^{-1} \otimes h_{0} \otimes \bar{\partial}\left(|\vec{\Phi}|^{2} v^{2}+\frac{1}{|\vec{\Phi}|^{2}} g \otimes|\alpha|^{2}\right) \\
& \quad-8 g^{-1} \otimes h_{0} \otimes \bar{\partial}\left(|\vec{\Phi}|^{2}\left\langle\vec{\Phi}, \vec{n}_{\vec{\Phi}}\right\rangle v^{2}+2 \operatorname{Re}(\alpha \otimes \partial \log |\vec{\Phi}|) v\right) \\
& \quad+4\left(\left\langle\vec{\Phi}, \vec{n}_{\vec{\Phi}}\right\rangle v+2 \operatorname{Re}(\alpha \otimes \partial \log |\vec{\Phi}|)\right)\left|h_{0}\right|_{W P}^{2} g \otimes \bar{\alpha} \\
& =4\left\langle\vec{\Phi}, \vec{n}_{\vec{\Phi}}\right\rangle g^{-1} \otimes h_{0} \otimes \bar{\partial}\left(|\vec{\Phi}|^{2} v^{2}+\frac{1}{|\vec{\Phi}|^{2}} g \otimes|\alpha|^{2}\right) \\
& \quad-8 g^{-1} \otimes h_{0} \otimes \bar{\partial}\left(|\vec{\Phi}|^{2}\left\langle\vec{\Phi}, \vec{n}_{\vec{\Phi}}\right\rangle v^{2}+2 \operatorname{Re}(\alpha \otimes \partial \log |\vec{\Phi}|)|\vec{\Phi}|^{2} v\right) \\
& \quad+4 K_{g}\left(\left\langle\vec{\Phi}, \vec{n}_{\vec{\Phi}}\right\rangle v+2 \operatorname{Re}(\alpha \otimes \partial \log |\vec{\Phi}|)\right) g \otimes \bar{\alpha}
\end{aligned}
$$

since $\left|\vec{h}_{0}\right|_{W P}^{2}=g^{-2} \otimes\left|\vec{h}_{0}\right|^{2}$. Finally, if

$$
\vec{u}=u \vec{n}_{\vec{\Phi}}+2 \operatorname{Re}(\alpha \otimes \vec{\Phi})
$$

we have by the proof of Lemma 10.3 since $\vec{H}=0$ and by the Codazzi identity

$$
\begin{aligned}
\Delta_{g}^{\perp}(\vec{u}-u \vec{n}) & =2 \operatorname{Re}\left(g^{-1} \otimes h_{0} \otimes \bar{\partial} \alpha\right) \vec{n} \\
\mathscr{A}(\vec{u}-u \vec{n}) & =-2 \operatorname{Re}\left(g^{-1} \otimes h_{0} \otimes \bar{\partial} \alpha\right)
\end{aligned}
$$

which implies that

$$
\begin{aligned}
\mathscr{L}_{g}^{\perp} \vec{u} & =\Delta_{g}^{\perp} \vec{u}+\mathscr{A}(\vec{u})=\left(\mathscr{L}_{g} u\right) \vec{n}=\left(\Delta_{g}-2 K_{g} u\right) \vec{n} \\
\Delta_{g}^{\perp} \vec{u}-\mathscr{A}(\vec{u}) & =\left(\Delta_{g} u+2 K_{g} u+4 \operatorname{Re}\left(g^{-1} \otimes h_{0} \otimes \bar{\partial} \alpha\right)\right) \vec{n} .
\end{aligned}
$$

By the proof of Lemma 10.3, we get

$$
\partial^{\perp} \vec{u}=\left(\partial u+\frac{1}{2} h_{0} \otimes \alpha\right) \vec{n} .
$$

Therefore, we have by (10.28) and (10.29)

$$
2\left\langle\Delta_{g}^{\perp} \vec{u}-\mathscr{A}(\vec{u}), \partial^{\perp} \vec{u}\right\rangle=\left(\Delta_{g} u+2 K_{g} u+4 \operatorname{Re}\left(g^{-1} \otimes h_{0} \otimes \bar{\partial} \alpha\right)\right)\left(2 \partial u+h_{0} \otimes \alpha\right) .
$$

Now, we directly have by (10.29)

$$
\partial\left|\nabla^{\perp} \vec{u}\right|_{g}^{2}=4 \partial\left|\partial^{\perp} \vec{u}\right|_{g}^{2}=\partial\left|2 \partial u+h_{0} \otimes \alpha\right|_{g}^{2} .
$$


Since $\vec{H}=0$, we have

$$
\begin{aligned}
\left\langle\partial_{z} \vec{\Phi}, \partial_{\bar{z}} \vec{u}\right\rangle & =\left\langle\partial_{z} \vec{\Phi}, \partial_{\bar{z}}\left(2 \operatorname{Re}\left(\alpha \partial_{z} \vec{\Phi}\right)\right)\right\rangle=\partial_{\bar{z}} \bar{\alpha} \frac{1}{2} e^{2 \lambda}+\bar{\alpha}\left\langle\partial_{z} \vec{\Phi}, \partial_{\bar{z}}^{2} \vec{\Phi}\right\rangle \\
& =\frac{1}{2} e^{2 \lambda} \partial_{\bar{z}} \bar{\alpha}+\frac{1}{2} \partial_{\bar{z}}\left(e^{2 \lambda}\right) \bar{\alpha}=\frac{1}{2} \partial_{\bar{z}}\left(e^{2 \lambda} \bar{\alpha}\right) .
\end{aligned}
$$

Now, we get by (10.29)

$$
\begin{aligned}
& 8 g^{-2} \otimes(\partial \vec{\Phi} \dot{\otimes} \bar{\partial} \vec{u}) \otimes\left(\vec{h}_{0} \dot{\otimes} \bar{\partial}^{\perp} \vec{u}\right) \\
& \quad=2 g^{-1} \otimes \bar{\partial}(g \otimes \bar{\alpha}) \otimes(2 \partial u)\left(2 g^{-1} \otimes h_{0} \otimes \bar{\partial} u+\left|h_{0}\right|_{W P}^{2} g \otimes \bar{\alpha}\right) \\
& \quad=2 g^{-1} \otimes \bar{\partial}(g \otimes \bar{\alpha}) \otimes\left(2 g^{-1} \otimes h_{0} \otimes \bar{\partial} u-K_{g} g \otimes \bar{\alpha}\right) .
\end{aligned}
$$

Finally, we have by (10.22), (10.27), (10.30), (10.31) and (10.32),

$$
\begin{aligned}
& \frac{d^{2}}{d t^{2}}\left(K_{g_{t}} d \operatorname{vol}_{g_{t}}\right)_{t=0}=d \operatorname{Im}\left(\left(\Delta_{g} u+2 K_{g} u+4 \operatorname{Re}\left(g^{-1} \otimes h_{0} \otimes \bar{\partial} \alpha\right)\right)\left(2 \partial u+h_{0} \otimes \alpha\right)\right. \\
& \quad-\partial\left|2 \partial u+h_{0} \otimes \alpha\right|_{g}^{2} \\
& \quad+2 g^{-1} \otimes \bar{\partial}(g \otimes \bar{\alpha}) \otimes\left(2 g^{-1} \otimes h_{0} \otimes \bar{\partial} u-K_{g} g \otimes \bar{\alpha}\right) \\
& \quad+4\left\langle\vec{\Phi}, \vec{n}_{\vec{\Phi}}\right\rangle g^{-1} \otimes h_{0} \otimes \bar{\partial}\left(|\vec{\Phi}|^{2} v^{2}+\frac{1}{|\vec{\Phi}|^{2}} g \otimes|\alpha|^{2}\right) \\
& \quad-8 g^{-1} \otimes h_{0} \otimes \bar{\partial}\left(|\vec{\Phi}|^{2}\left\langle\vec{\Phi}, \vec{n}_{\vec{\Phi}}\right\rangle v^{2}+2 \operatorname{Re}(\alpha \otimes \partial \log |\vec{\Phi}|)|\vec{\Phi}|^{2} v\right) \\
& \left.\quad+4 K_{g}\left(\left\langle\vec{\Phi}, \vec{n}_{\vec{\Phi}}\right\rangle v+2 \operatorname{Re}(\alpha \otimes \partial \log |\vec{\Phi}|)\right) g \otimes \bar{\alpha}\right)
\end{aligned}
$$

Using Lemma 10.3, we deduce

$$
\frac{d}{d t} \vec{H}_{t}=\frac{1}{2} \mathscr{L}_{g}^{\perp} \vec{u}=\frac{1}{2}\left(\Delta_{g}^{\perp} \vec{u}+\mathscr{A}(\vec{u})\right)=\frac{1}{2}\left(\Delta_{g} u-2 K_{g} u\right)=\frac{1}{2} \mathscr{L}_{g} u,
$$

and this concludes the proof of the theorem by the pointwise conformal invariance of $\left(H^{2}-\right.$ $\left.K_{g}\right) d \operatorname{vol}_{g}$.

\subsection{Formulae for inversions of (minimal) surfaces}

Lemma 10.6 Let $\vec{\Phi}: \Sigma \backslash\left\{p_{1}, \ldots, p_{n}\right\} \rightarrow \mathbb{R}^{3}$ be a complete minimal surface, and let $(g, \omega)$ be its Weierstrass data. Then the Weingarten holomorphic quadratic differential is given by

$$
h_{0}=-2 \partial g \otimes \omega .
$$

Proof Write locally $\omega=f(z) d z$. Then we have by [11]

$$
\begin{aligned}
\partial_{z} \vec{\Phi} & =\frac{1}{2}\left(1-g^{2}, i\left(1+g^{2}\right), 2 g\right) f(z) \\
\partial_{z}^{2} \vec{\Phi} & =g^{\prime}(z)(-2 g(z), 2 i g(z), 2) f(z)+\frac{1}{2}\left(1-g^{2},\left(i+g^{2}\right), 2 g\right) f^{\prime}(z) \\
\vec{n} & =\left(\frac{2 \operatorname{Re}(g)}{1+|g|^{2}}, \frac{2 \operatorname{Im}(g)}{1+|g|^{2}}, \frac{-1+|g(z)|^{2}}{1+|g(z)|^{2}}\right) \\
h_{0} & =2\left\langle\partial_{z}^{2} \vec{\Phi}, \vec{n}\right\rangle d z^{2}=2 \frac{g^{\prime}(z)}{1+|g(z)|^{2}}(-g(z)(g(z)+\overline{g(z)})
\end{aligned}
$$




$$
\begin{aligned}
& \left.+g(z)(g(z)-\overline{g(z)})-1+|g(z)|^{2}\right) f(z) d z^{2} \\
= & -2 g^{\prime}(z) f(z) d z^{2}=-2 \partial g \otimes \omega,
\end{aligned}
$$

and this concludes the proof of the lemma.

Lemma 10.7 Let $\vec{\Phi}: \Sigma \rightarrow \mathbb{R}^{3}$ be an immersion, and define $\vec{\Psi}: \Sigma \backslash \vec{\Phi}^{-1}(\{0\}) \rightarrow \mathbb{R}^{3}$ by

$$
\vec{\Psi}=\frac{\vec{\Phi}}{|\vec{\Phi}|^{2}} \text {. }
$$

If $\vec{n}_{\vec{\Phi}}: \Sigma \rightarrow S^{2}$ is the normal unit of $\vec{\Phi}$, then the normal unit $\vec{n}_{\vec{\Psi}}: \Sigma \backslash \vec{\Phi}^{-1}(\{0\}) \rightarrow S^{2}$ of $\vec{\Psi}$ is given by

$$
\vec{n}_{\vec{\Psi}}=-\left(\vec{n}_{\vec{\Phi}}-2\left\langle\vec{n}_{\vec{\Phi}}, \vec{\Phi}\right\rangle \frac{\vec{\Phi}}{|\vec{\Phi}|^{2}}\right)
$$

Proof Recall the identities valid for all $(a, b, c) \in \mathbb{R}^{3}$

$$
\left\{\begin{aligned}
a \times a & =0 \\
a \times(b \times c) & =\langle a, c\rangle b-\langle a, b\rangle c .
\end{aligned}\right.
$$

We also recall the formula

$$
\vec{n}_{\vec{\Psi}}=\frac{\partial_{x} \vec{\Psi} \times \partial_{y} \vec{\Psi}}{\left|\partial_{x} \vec{\Psi} \times \partial_{y} \vec{\Psi}\right|}
$$

To simplify computations, assume that we are given a conformal parametrisation of $\vec{\Phi}$, and let $\lambda$ be the conformal parameter of $\vec{\Phi}$ given by

$$
e^{2 \lambda}=\left|\partial_{x} \vec{\Phi} \times \partial_{y} \vec{\Phi}\right|=\left|\partial_{x} \vec{\Phi}\right|^{2}=\left|\partial_{y} \vec{\Phi}\right|^{2} .
$$

Then we have

$$
\partial_{z} \vec{\Psi}=\frac{\partial_{z} \vec{\Phi}}{|\vec{\Phi}|^{2}}-2 \frac{\left\langle\partial_{z} \vec{\Phi}, \vec{\Phi}\right\rangle}{|\vec{\Phi}|^{4}} \vec{\Phi}
$$

which implies that

$$
\left|\partial_{z} \vec{\Psi}\right|^{2}=\frac{\left|\partial_{z} \vec{\Phi}\right|^{2}}{|\vec{\Phi}|^{4}}-4 \operatorname{Re}\left(\frac{\left\langle\partial_{z} \vec{\Phi}, \vec{\Phi}\right\rangle}{|\vec{\Phi}|^{6}}\left\langle\partial_{z} \vec{\Phi}, \vec{\Phi}\right\rangle\right)+4 \frac{\left|\left\langle\partial_{z} \vec{\Phi}, \vec{\Phi}\right\rangle\right|^{2}}{|\vec{\Phi}|^{8}}|\vec{\Phi}|^{2}=\frac{\left|\partial_{z} \vec{\Phi}\right|^{2}}{|\vec{\Phi}|^{4}}=\frac{1}{2} \frac{e^{2 \lambda}}{|\vec{\Phi}|^{4}} .
$$

Therefore, the conformal parameter $\mu$ of $\vec{\Psi}$ is given by

$$
e^{2 \mu}=\frac{e^{2 \lambda}}{|\vec{\Phi}|^{4}}
$$

Now, we compute

$$
\begin{aligned}
e^{2 \mu} \vec{n}_{\vec{\Psi}} & =\left(\frac{\partial_{x} \vec{\Phi}}{|\vec{\Phi}|^{2}}-2 \frac{\left\langle\partial_{x} \vec{\Phi}, \vec{\Phi}\right\rangle}{|\vec{\Phi}|^{4}} \vec{\Phi}\right) \times\left(\frac{\partial_{y} \vec{\Phi}}{|\vec{\Phi}|^{2}}-2 \frac{\left\langle\partial_{y} \vec{\Phi}, \vec{\Phi}\right\rangle}{|\vec{\Phi}|^{4}} \vec{\Phi}\right) \\
& =\frac{\partial_{x} \vec{\Phi} \times \partial_{y} \vec{\Phi}}{|\vec{\Phi}|^{4}}-2 \frac{\left\langle\partial_{y} \vec{\Phi}, \vec{\Phi}\right\rangle}{|\vec{\Phi}|^{6}}\left(\partial_{x} \vec{\Phi} \times \vec{\Phi}\right)-2 \frac{\left\langle\partial_{x} \vec{\Phi}, \vec{\Phi}\right\rangle}{|\vec{\Phi}|^{6}}\left(\vec{\Phi} \times \partial_{y} \vec{\Phi}\right)
\end{aligned}
$$




$$
=e^{2 \mu} \vec{n}_{\vec{\Phi}}-2 \frac{\left\langle\partial_{y} \vec{\Phi}, \vec{\Phi}\right\rangle}{|\vec{\Phi}|^{6}}\left(\partial_{x} \vec{\Phi} \times \vec{\Phi}\right)-2 \frac{\left\langle\partial_{x} \vec{\Phi}, \vec{\Phi}\right\rangle}{|\vec{\Phi}|^{6}}\left(\vec{\Phi} \times \partial_{y} \vec{\Phi}\right)
$$

Trivially, we have

$$
\vec{\Phi}=e^{-2 \lambda}\left\langle\partial_{x} \vec{\Phi}, \vec{\Phi}\right\rangle \partial_{x} \vec{\Phi}+e^{-2 \lambda}\left\langle\partial_{y} \vec{\Phi}, \vec{\Phi}\right\rangle \partial_{y} \vec{\Phi}+\left\langle\vec{\Phi}, \vec{n}_{\vec{\Phi}}\right\rangle \vec{n}_{\vec{\Phi}}
$$

Since $\vec{n}_{\vec{\Phi}}=e^{-2 \lambda} \partial_{x} \vec{\Phi} \times \partial_{y} \vec{\Phi}$, we deduce by (10.33) that

$$
\begin{aligned}
& \partial_{x} \vec{\Phi} \times \vec{n}_{\vec{\Phi}}=e^{-2 \lambda}\left(\left\langle\partial_{x} \vec{\Phi}, \partial_{y} \vec{\Phi}\right\rangle \partial_{x} \vec{\Phi}-\left\langle\partial_{x} \vec{\Phi}, \partial_{x} \vec{\Phi}\right\rangle \partial_{y} \vec{\Phi}\right)=-\partial_{y} \vec{\Phi} \\
& \partial_{y} \vec{\Phi} \times \vec{n}_{\vec{\Phi}}=e^{-2 \lambda}\left(\left\langle\partial_{y} \vec{\Phi}, \partial_{y} \vec{\Phi}\right\rangle \partial_{x} \vec{\Phi}-\left\langle\partial_{y} \vec{\Phi}, \partial_{x} \vec{\Phi}\right\rangle \partial_{x} \vec{\Phi}\right)=\partial_{x} \vec{\Phi}
\end{aligned}
$$

Therefore, we have

$$
\begin{aligned}
& \partial_{x} \vec{\Phi} \times \vec{\Phi}=\left\langle\partial_{y} \vec{\Phi}, \vec{\Phi}\right\rangle \vec{n}_{\vec{\Phi}}-\left\langle\vec{\Phi}, \vec{n}_{\vec{\Phi}}\right\rangle \partial_{y} \vec{\Phi} \\
& \vec{\Phi} \times \partial_{y} \vec{\Phi}=\left\langle\partial_{x} \vec{\Phi}, \vec{\Phi}\right\rangle \vec{n}_{\vec{\Phi}}-\left\langle\vec{\Phi}, \vec{n}_{\vec{\Phi}}\right\rangle \partial_{x} \vec{\Phi}
\end{aligned}
$$

Finally, we get

$$
\begin{aligned}
\vec{n}_{\vec{\Psi}}= & \vec{n}_{\vec{\Phi}}-2 e^{-2 \lambda} \frac{\left\langle\partial_{y} \vec{\Phi}, \vec{\Phi}\right\rangle}{|\vec{\Phi}|^{2}}\left(\left\langle\partial_{y} \vec{\Phi}, \vec{\Phi}\right\rangle \vec{n}_{\vec{\Phi}}-\left\langle\vec{\Phi}, \vec{n}_{\vec{\Phi}}\right\rangle \partial_{y} \vec{\Phi}\right) \\
& -2 e^{-2 \lambda} \frac{\left\langle\partial_{x} \vec{\Phi}, \vec{\Phi}\right\rangle}{|\vec{\Phi}|^{2}}\left(\left\langle\partial_{x} \vec{\Phi}, \vec{\Phi}\right\rangle \vec{n}_{\vec{\Phi}}-\left\langle\vec{\Phi}, \vec{n}_{\vec{\Phi}}\right\rangle \partial_{x} \vec{\Phi}\right) \\
= & \vec{n}_{\vec{\Phi}}-\frac{2}{|\vec{\Phi}|^{2}}\left(e^{-2 \lambda}\left\langle\partial_{y} \vec{\Phi}, \vec{\Phi}\right\rangle^{2}+e^{-2 \lambda}\left\langle\partial_{x} \vec{\Phi}, \vec{\Phi}\right\rangle^{2}\right) \vec{n}_{\vec{\Phi}} \\
& +\frac{2}{|\vec{\Phi}|^{2}}\left\langle\vec{\Phi}, \vec{n}_{\vec{\Phi}}\right\rangle\left(e^{-2 \lambda}\left\langle\partial_{y} \vec{\Phi}, \vec{\Phi}\right\rangle \partial_{y} \vec{\Phi}+e^{-2 \lambda}\left\langle\partial_{x} \vec{\Phi}, \vec{\Phi}\right\rangle \partial_{x} \vec{\Phi}\right) \\
= & \vec{n}_{\vec{\Phi}}-\frac{2}{|\vec{\Phi}|^{2}}\left(e^{-2 \lambda}\left\langle\partial_{y} \vec{\Phi}, \vec{\Phi}\right\rangle^{2}+e^{-2 \lambda}\left\langle\partial_{x} \vec{\Phi}, \vec{\Phi}\right\rangle^{2}+\left\langle\vec{\Phi}, \vec{n}_{\vec{\Phi}}\right\rangle^{2}\right) \vec{n}_{\vec{\Phi}}+2\left\langle\vec{\Phi}, \vec{n}_{\vec{\Phi}}\right\rangle \frac{\vec{\Phi}}{|\vec{\Phi}|^{2}} \\
= & -\vec{n}_{\vec{\Phi}}+2\left\langle\vec{\Phi}, \vec{n}_{\vec{\Phi}}\right\rangle \frac{\vec{\Phi}}{|\vec{\Phi}|^{2}}
\end{aligned}
$$

since $|\vec{\Phi}|^{2}=e^{-2 \lambda}\left\langle\partial_{x} \vec{\Phi}, \vec{\Phi}\right\rangle^{2}+e^{-2 \lambda}\left\langle\partial_{y} \vec{\Phi}, \vec{\Phi}\right\rangle^{2}+\left\langle\vec{\Phi}, \vec{n}_{\vec{\Phi}}\right\rangle^{2}$.

Lemma 10.8 Let $\vec{\Phi}: \Sigma \backslash\left\{p_{1}, \ldots, p_{n}\right\} \rightarrow \mathbb{R}^{3}$ be a smooth immersion and $\vec{\Psi}=\frac{\vec{\Phi}}{|\vec{\Phi}|^{2}}: \Sigma \rightarrow$ $\mathbb{R}^{n}$ be its inversion. Then we have

$$
\left\{\begin{array}{l}
H_{\vec{\Psi}}=-|\vec{\Phi}|^{2} H_{\vec{\Phi}}-2\left\langle\vec{n}_{\vec{\Phi}}, \vec{\Phi}\right\rangle \\
h_{\vec{\Psi}}^{0}=-\frac{1}{|\vec{\Phi}|^{2}} h_{\vec{\Phi}}^{0}
\end{array}\right.
$$

In particular, if $\vec{\Phi}$ is minimal $\left(H_{\vec{\Phi}}=0\right)$, we have $H_{\vec{\Psi}}=-2\left\langle\vec{n}_{\vec{\Phi}}, \vec{\Phi}\right\rangle$.

Proof We have

$$
\begin{aligned}
\partial_{\bar{z}} \vec{\Psi} & =\frac{\partial_{\bar{z}} \vec{\Phi}}{|\vec{\Phi}|^{2}}-\frac{2\left\langle\partial_{\bar{z}} \vec{\Phi}, \vec{\Phi}\right\rangle}{|\vec{\Phi}|^{4}} \vec{\Phi} \quad \text { and } \quad e^{2 \mu}=2\left|\partial_{z} \vec{\Psi}\right|^{2}=\frac{2\left|\partial_{z} \vec{\Phi}\right|^{2}}{|\vec{\Phi}|^{4}}=\frac{e^{2 \lambda}}{|\vec{\Phi}|^{4}} \\
\partial_{z \bar{z}}^{2} \vec{\Psi} & =\frac{\partial_{z \bar{z}}^{2} \vec{\Phi}}{|\vec{\Phi}|^{2}}-4 \operatorname{Re}\left(\frac{\left\langle\partial_{\bar{z}} \vec{\Phi}, \vec{\Phi}\right\rangle}{|\vec{\Phi}|^{4}} \partial_{z} \vec{\Phi}\right)-\frac{2\left\langle\partial_{z \bar{z}}^{2} \vec{\Phi}, \vec{\Phi}\right\rangle}{|\vec{\Phi}|^{4}} \vec{\Phi}-\frac{2\left\langle\partial_{\bar{z}} \vec{\Phi}, \partial_{z} \vec{\Phi}\right\rangle}{|\vec{\Phi}|^{4}} \vec{\Phi}+\frac{8\left|\left\langle\partial_{z} \vec{\Phi}, \vec{\Phi}\right\rangle\right|^{2}}{|\vec{\Phi}|^{6}} \vec{\Phi}
\end{aligned}
$$




$$
\begin{aligned}
= & \frac{1}{2} \frac{e^{2 \lambda}}{|\vec{\Phi}|^{2}} \vec{H}_{\vec{\Phi}}-4 \operatorname{Re}\left(\frac{\left\langle\partial_{\bar{z}} \vec{\Phi}, \vec{\Phi}\right\rangle}{|\vec{\Phi}|^{4}} \partial_{z} \vec{\Phi}\right)+\left(-e^{2 \mu}\left\langle\vec{H}_{\vec{\Phi}}, \vec{\Phi}\right\rangle-e^{2 \mu}+\frac{8}{|\vec{\Phi}|^{2}}\left|\partial_{z} \log \right| \vec{\Phi}||^{2}\right) \vec{\Phi} \\
\vec{H}_{\vec{\Psi}}= & 2 e^{-2 \mu} \partial_{z \bar{z}}^{2} \vec{\Psi}=|\vec{\Phi}|^{2} \vec{H}_{\vec{\Phi}}-8 \operatorname{Re}\left(e^{-2 \lambda}\left\langle\partial_{\bar{z}} \vec{\Phi}, \vec{\Phi}\right\rangle \partial_{z} \vec{\Phi}\right) \\
& +\left(-2-2\left\langle\vec{H}_{\vec{\Phi}}, \vec{\Phi}\right\rangle+16|\vec{\Phi}|^{2} e^{-2 \lambda}\left|\partial_{z} \log \right| \vec{\Phi}||^{2}\right) \vec{\Phi} \\
= & |\vec{\Phi}|^{2} \vec{H}_{\vec{\Phi}}-\left\langle d \vec{\Phi}, d|\vec{\Phi}|^{2}\right\rangle_{g}+\left(-2-2\left\langle\vec{H}_{\vec{\Phi}}, \vec{\Phi}\right\rangle+4|\vec{\Phi}|^{2}|d \log | \vec{\Phi}||_{g}^{2}\right) \vec{\Phi}
\end{aligned}
$$

Therefore, we have by Lemma 10.7

$$
\begin{aligned}
& -\left\langle\vec{H}_{\vec{\Psi}}, \vec{n}_{\vec{\Phi}}\right\rangle=-|\vec{\Phi}|^{2} H_{\vec{\Phi}}+\left(2-\left.16|\vec{\Phi}|^{2}|\partial \log | \vec{\Phi}\right|_{g} ^{2}\right)\left\langle\vec{n}_{\vec{\Phi}}, \vec{\Phi}\right\rangle+2\left\langle\vec{H}_{\vec{\Phi}}, \vec{\Phi}\right\rangle\left\langle\vec{n}_{\vec{\Phi}}, \vec{\Phi}\right\rangle \\
& =\left(-|\vec{\Phi}|^{2}+2\left\langle\vec{n}_{\vec{\Phi}}, \vec{\Phi}\right\rangle^{2}\right) H_{\vec{\Phi}}+\left(2-16|\vec{\Phi}|^{2}|\partial \log | \vec{\Phi}||_{g}^{2}\right)\left\langle\vec{n}_{\vec{\Phi}}, \vec{\Phi}\right\rangle \\
& 2\left\langle\vec{n}_{\vec{\Phi}}, \vec{\Phi}\right\rangle\left\langle\vec{H}_{\vec{\Psi}}, \frac{\vec{\Phi}}{|\vec{\Phi}|^{2}}\right\rangle=2\left\langle\vec{n}_{\vec{\Phi}}, \vec{\Phi}\right\rangle^{2} H_{\vec{\Phi}}-\left.16\left\langle\vec{n}_{\vec{\Phi}}, \vec{\Phi}\right\rangle|\vec{\Phi}|^{2}|\partial \log | \vec{\Phi}\right|_{g} ^{2} \\
& \quad+2\left(-2-2 H_{\vec{\Phi}}\left\langle\vec{n}_{\vec{\Phi}}, \vec{\Phi}\right\rangle+\left.16|\vec{\Phi}|^{2}|\partial \log | \vec{\Phi}\right|_{g} ^{2}\right)\left\langle\vec{n}_{\vec{\Phi}}, \vec{\Phi}\right\rangle \\
& =-2\left\langle\vec{n}_{\vec{\Phi}}, \vec{\Phi}\right\rangle^{2} H_{\vec{\Phi}}+\left(-4+16|\vec{\Phi}|^{2}|\partial \log | \vec{\Phi}||_{g}^{2}\right)\left\langle\overrightarrow{n_{\vec{\Phi}}}, \vec{\Phi}\right\rangle \\
& H_{\vec{\Psi}}=\left\langle\vec{H}_{\vec{\Psi}}, \vec{n}_{\vec{\Psi}}\right\rangle=\left\langle\vec{H}_{\vec{\Psi}},-\vec{n}_{\vec{\Phi}}+2\left\langle\overrightarrow{n_{\vec{\Phi}}}, \vec{\Phi}\right\rangle \frac{\vec{\Phi}}{|\vec{\Phi}|^{2}}\right\rangle=-|\vec{\Phi}|^{2} H_{\vec{\Phi}}-2\left\langle\vec{n}_{\vec{\Phi}}, \vec{\Phi}\right\rangle .
\end{aligned}
$$

Likewise, we have using $\left\langle\partial_{z} \vec{\Phi}, \partial_{z} \vec{\Phi}\right\rangle=0$

$$
\begin{aligned}
\partial_{z}^{2} \vec{\Psi}= & \frac{\partial_{z}^{2} \vec{\Phi}}{|\vec{\Phi}|^{2}}-\frac{4\left\langle\partial_{z} \vec{\Phi}, \vec{\Phi}\right\rangle}{|\vec{\Phi}|^{4}} \partial_{z} \vec{\Phi}-\frac{2\left\langle\partial_{z}^{2} \vec{\Phi}, \vec{\Phi}\right\rangle}{|\vec{\Phi}|^{4}} \vec{\Phi}+\frac{8\left\langle\partial_{z} \vec{\Phi}, \vec{\Phi}\right\rangle^{2}}{|\vec{\Phi}|^{6}} \vec{\Phi} \\
= & \frac{1}{2|\vec{\Phi}|^{2}} \vec{h}_{\vec{\Phi}}^{0}+\left(2\left(\partial_{z} \lambda\right)-4 \partial_{z} \log |\vec{\Phi}|\right) \frac{\partial_{z} \vec{\Phi}}{|\vec{\Phi}|^{2}} \\
& +\left(-\frac{\left\langle\vec{h}_{\vec{\Phi}}^{0}, \vec{\Phi}\right\rangle}{|\vec{\Phi}|^{4}}-4\left(\partial_{z} \lambda\right) \frac{\partial_{z} \log |\vec{\Phi}|}{|\vec{\Phi}|^{2}}+\frac{8\left(\partial_{z} \log |\vec{\Phi}|\right)^{2}}{|\vec{\Phi}|^{2}}\right) \vec{\Phi} \\
2\left(\partial_{z} \mu\right)= & 2\left(\partial_{z} \lambda\right)-4 \partial_{z} \log |\vec{\Phi}| \\
\vec{h}_{\vec{\Psi}}^{0}= & 2\left(\partial_{z}^{2} \vec{\Psi}-2\left(\partial_{z} \lambda\right) \partial_{z} \vec{\Psi}\right)=\frac{1}{|\vec{\Phi}|^{2}} \vec{h}_{\vec{\Phi}}^{0}-2\left\langle\vec{\Phi}, \vec{h}_{\vec{\Phi}}^{0}\right\rangle \frac{\vec{\Phi}}{|\vec{\Phi}|^{4}} \\
= & \left(\vec{n}_{\vec{\Phi}}-2\left\langle\vec{\Phi}, \vec{n}_{\vec{\Phi}}\right\rangle \frac{\vec{\Phi}}{|\vec{\Phi}|^{2}}\right) \frac{1}{|\vec{\Phi}|^{2}} h_{\vec{\Phi}}^{0}=-\frac{1}{|\vec{\Phi}|^{2}} h_{\vec{\Phi}}^{0} \overrightarrow{n_{\Psi}}
\end{aligned}
$$

which implies the second identity [see also (3.66 in [29])].

Remark 10.9 We can easily see that those formulae are consistent with the Codazzi identity. Indeed, we first have

$$
\partial_{z} \vec{n}_{\vec{\Phi}}=-H \partial_{z} \vec{\Phi}-e^{-2 \lambda} h_{\vec{\Phi}}^{0} \partial_{\bar{z}} \vec{\Phi}
$$

and the Codazzi identity $\bar{\partial} h_{\vec{\Phi}}^{0}=g_{\vec{\Phi}} \otimes \partial H_{\vec{\Phi}}$ implies that

$$
\partial H_{\vec{\Psi}}=-2\left\langle\partial_{z} \vec{\Phi}, \vec{\Phi}\right\rangle H_{\vec{\Phi}} d z-|\vec{\Phi}|^{2} \partial H_{\vec{\Phi}}-2\left\langle\vec{\Phi},-H_{\vec{\Phi}} \partial_{z} \vec{\Phi}-e^{-2 \lambda} h_{\vec{\Phi}}^{0} \partial_{\bar{z}} \vec{\Phi}\right\rangle d z
$$




$$
\begin{aligned}
& =-|\vec{\Phi}|^{2} g_{\vec{\Phi}}^{-1} \otimes \bar{\partial} h_{\vec{\Phi}}^{0}+2 g_{\vec{\Phi}}^{-1} \otimes\langle\bar{\partial} \vec{\Phi}, \vec{\Phi}\rangle \otimes h_{\vec{\Phi}}^{0} \\
\bar{\partial} h_{\vec{\Psi}}^{0} & =\frac{2\langle\bar{\partial} \vec{\Phi}, \vec{\Phi}\rangle}{|\vec{\Phi}|^{4}} \otimes h_{\vec{\Phi}}^{0}-\frac{1}{|\vec{\Phi}|^{2}} \bar{\partial} h_{\vec{\Phi}}^{0} \\
& =\frac{1}{|\vec{\Phi}|^{4}} g_{\vec{\Phi}} \otimes\left(-|\vec{\Phi}|^{2} g_{\vec{\Phi}}^{-1} \otimes \bar{\partial} h_{\vec{\Phi}}^{0}+2 g_{\vec{\Phi}}^{-1} \otimes\langle\bar{\partial} \vec{\Phi}, \vec{\Phi}\rangle \otimes h_{\vec{\Phi}}^{0}\right)=g_{\vec{\Psi}} \otimes \partial H_{\vec{\Psi}} .
\end{aligned}
$$

Furthermore, the formula in $H_{\vec{\Psi}}$ is symmetric. Indeed, if $\vec{\Phi}$ is minimal, then $H_{\vec{\Psi}}=$ $-2\left\langle\vec{n}_{\vec{\Phi}}, \vec{\Phi}\right\rangle$. Now, if $\vec{\Psi}$ is minimal, we have $|\vec{\Phi}|^{2} H_{\vec{\Phi}}+2\left\langle\vec{n}_{\vec{\Phi}}, \vec{\Phi}\right\rangle=0$, and Lemma 10.7 implies that

$$
H_{\vec{\Phi}}=-2\left\langle\vec{n}_{\vec{\Phi}}, \frac{\vec{\Phi}}{|\vec{\Phi}|^{2}}\right\rangle=-2\left\langle-\vec{n}_{\vec{\Psi}}+2\left\langle\vec{n}_{\vec{\Psi}}, \vec{\Psi}\right\rangle \frac{\vec{\Psi}}{|\vec{\Psi}|^{2}}, \vec{\Psi}\right\rangle=-2\left\langle\vec{n}_{\vec{\Psi}}, \vec{\Psi}\right\rangle
$$

\section{References}

1. Bernard, Y., Rivière, T.: Singularity removability at branch points for Willmore surfaces. Pac. J. Math. 265(2), 257-311 (2013)

2. Bernard, Y., Rivière, T.: Energy quantization for Willmore surfaces and applications. Ann. Math. 180, 87-136 (2014)

3. Bethuel, F., Brezis, H., Hélein, F.: Asymptotics for the minimization of a Ginzburg-Landau functional. Calc. Var. Partial Differ. Equ. 1(2), 123-148 (1993)

4. Bethuel, F., Brezis, H.: Hélein Ginzburg-Landau Vortices. Birkhäuser, Frédéric (1994)

5. Blaschke, W.J.E.: Vorlesungen Über Differentialgeometrie III: Differentialgeometrie der Kreise und Kugeln, Springer, coll. Grundlehren der mathematischen Wissenschaften (1929)

6. Bonnet, P.-O.: Mémoire sur la théorie générale des surfaces. J. l'École Polytech. 32, 1-46 (1848)

7. Brezis, H.: Analyse fonctionnelle. Théorie et applications, Collection Mathématiques Appliquées pour la Maîtrise. Masson, Paris (1983)

8. Bryant, R.L.: A duality theorem for Willmore surfaces. J. Differ. Geom. 20, 23-53 (1984)

9. Choe, J.: Index, vision number and stability of complete minimal surfaces. Arch. Rational Mech. Anal. 109(3), 195-212 (1990)

10. Costa, C.J.: Complete minimal surfaces in $\mathbb{R}^{3}$ of genus one and four planar embedded ends. Proc. Am. Math. Soc. 119(4), 1279-1287 (1993)

11. Dierkes, U., Hildebrandt, S., Sauvigny, F.: Minimal Surfaces. Grundlehren der Mathematischen Wissenschaften, vol. 339. Springer, Berlin (2010)

12. Euler, L.: Recherches sur la courbure des surfaces. Mém. l'acad. Sci. Berlin 16, 119-143 1767, (1760)

13. Farb, B., Margalit, D.: A Primer on Mapping Class Groups, Princeton Mathematical Series, vol. 49. Princeton University Press, Princeton (2012)

14. Gauss, C.F.: Disquisitiones generales circa superficies curvas. Comment. Soc. Regiae Sci. Gottingensis Recentiores 6, 99-146 (1827)

15. Germain, S.: Mémoire sur la coubure des surfaces. J. Reine Angew. Math. 7, 1-29 (1831)

16. Germain, S., Mémoire sur cette question proposée par la premiere classe de l'institut : Donner la théorie mathématique des vibrations des surfaces élastiques, et la comparer à l'expérience, Académie des Sciences, 30 septembre 1815

17. Hirsch, J., Mäder-Baumdicker, E.: On the Index of Willmore spheres (2019). arXiv:1905.04185

18. Jorge, L.P., Meeks III, W.H.: The topology of complete minimal surfaces of finite total Gaussian curvature. Topology 22(2), 203-221 (1983)

19. Kusner, R.: On the Minimax Sphere Eversion. Unpublished note (1995)

20. Kusner, R., Schmitt, N.: The Spinor Representation of Surfaces in Space (1996). arXiv:dg-ga/9610005

21. Li, P., Yau, S.-T.: A new conformal invariant and its applications to the willmore conjecture and the first eigenvalue of compact surfaces. Invent. Math. 69, 269-291 (1982)

22. McMullen, C.T.: The Moduli space of Riemann surfaces is Kähler hyperbolic. Ann. Math. 151(1), 327357 (2000)

23. Marques, F.C., Neves, A.: Min-Max theory and the Willmore conjecture. Ann. Math. 179, 683-782 (2014) 
24. Meusnier, J.-B.: Mémoire sur la courbure des surfaces. Mém. Math. Phys. Acad. Sci. Paris Prés. Par Div. Savans 10, 477-510, 1785 (1776)

25. Michelat, A.: On the Morse Index of Critical Points in the Viscosity Method (2018). arXiv:1806.09578

26. Michelat, A.: Morse Index of Min-Max Willmore Surfaces (2018). arXiv:1808.07700

27. Michelat, A.: Morse-Theoretic Aspects of the Willmore Energy. ETH, Zürich (2019)

28. Michelat, A.: On the Morse index of Willmore spheres in $S^{3}$. Commun. Anal. Geom. 28(6), 1337-1406 (2020)

29. Michelat, A., Rivière, T.: The classification of branched Willmore spheres in the 3 -sphere and the 4-sphere. Ann. Sci. Éc. Norm. Supér. (2021)+ (to appear)

30. Mondino, A., Nguyen, H.: The global conformal invariants for submanifolds. Ann. Inst. Fourier (Grenoble) 68(6), 2663-2695 (2018)

31. Navier, H.: Mémoire sur la flexion des plans élastiques. In: Presented at the Institut the 14th of May 1821 (1820)

32. Pacard, F., Rivière, T.: Linear and nonlinear aspects of vortices. The Ginzburg-Landau Model, Birkäuser (2000)

33. Poisson, S.D.: Mémoire sur les surfaces élastiques. Mémoire de l'Institut (1814)

34. Rivière, T.: Analysis aspects of Willmore surfaces. Invent. Math. 174, 1-45 (2008)

35. Rivière, T.: Variational principles for immersed surfaces with $L^{2}$-bounded second fundamental form. J. Reine Angew. Math. 695, 41-98 (2014)

36. Rivière, T.: Willmore minmax surfaces and the cost of the sphere eversion. J. Eur. Math. Soc. 23(2), 349-423 (2021)

37. Rodrigues, O.: Recherches sur la théorie des intégrales doubles et des rayons de courbure des surfaces, et sur la transformation d'une classe d'intégrales doubles qui ont un rapport direct avec les formules de cette théorie. Corresp. l'École Polyetch. 3, 162-183 (1815)

38. Rodrigues, O.: Sur quelques propriétés des intégrales doubles et des rayons de courbure des surfaces. Bull. Sci. Soc. Philomatique 2, 34-36 (1815)

39. Schoen, R.M.: Uniqueness, symmetry, and embeddedness of minimal surfaces. J. Differ. Geom. 18, 791-809 (1983)

40. Serfaty, S.: Coulomb Gases and Ginzburg-Landau Vortices, Zurich Lectures in Advanced Mathematics. European Mathematical Society (EMS), Zürich (2015)

41. Shamaev, È.I.: On a family of minimal tori in $\mathbb{R}^{3}$ with planar ends. Sibirsk. Mat. Zh. 46, no. 6, 1407-1426 (Translation in Siberian Math. J. 46, no. 6, 1135-1152) (2005)

42. Simons, J.: Minimal varieties in Riemannian manifolds. Ann. Math. Second Ser. 88(1), 62-105 (1968)

43. Smale, S.: A classification of immersions of the two-sphere. Trans. Am. Math. Soc. 90, 281-290 (1958)

44. Smale, S.: On the Morse index theorem. J. Math. Mech. 14(6), 1049-1055 (1965)

45. Thomsen, G.: Über konforme Geometrie, I: Grundlagen der konformen Flächentheorie. Abh. Math. Semin. Univ. Hamb. 3, 31 (1924)

46. Todhunter, I.: A history of the theory of elasticity and the strength of materials, from Galilei to Lord Kelvin. In: Edited and completed by Karl Pearson Dover Publications, Inc., New York 1960 vol. I: xvi+936 pp.; Vol. II, Part I: xiii+762 pp. (4 plates); Vol. II, Part II: iv+546 pp. (1894)

47. Willmore, T.J.: Note on embedded surfaces. An. st. Univ. Ias S.I.a. Math. 11B, 493-496 (1965)

Publisher's Note Springer Nature remains neutral with regard to jurisdictional claims in published maps and institutional affiliations. 